\title{
Bacterial diversity and viral discovery in the invasive Argentine ant (Linepithema humile)
}

by

Alexandra Sébastien

A thesis submitted to

Victoria University of Wellington

in fulfilment of the requirements for the degree of

Doctor of Philosophy in Ecology

Victoria University of Wellington

2016 
This thesis was conducted under the supervision of:

\section{Dr. Philip J. Lester (Primary supervisor)}

Victoria University of Wellington,

Wellington, New Zealand

and

Dr. Peter A. Ritchie (Secondary supervisor)

Victoria University of Wellington,

Wellington, New Zealand 


\section{Abstract}

Invasive species can lead to major economic and ecological issues. For this reason, biological controls are being developed in order to help with invasive species population management. Pathogenic bacteria and viruses offer good biological control opportunities as both micro-organisms have played a role in natural population declines. However, beneficial bacteria and viruses associated with the targeted invasive species may interfere with biological controls, by protecting their hosts from infections. Previous knowledge on both pathogenic and beneficial bacteria and viruses present in invasive species may then support the development of an active and efficient biological control.

The Argentine ant, Linepithema humile, is a South American invasive ant species that has successfully spread over five continents. The ants were introduced to New Zealand after a complex invasion path, from Argentina their home range to Europe, then to Australia and finally to New Zealand. In their new environments, invasive Argentine ants affect species diversity and can cause agricultural losses. In the absence of any biological controls, the Argentine ant population is controlled by chemical sprays and poison baits. Management of these invasive ants in New Zealand is estimated to cost NZ\$ 60 million a year. The Argentine ant population in New Zealand was reported to have unexpectedly declined. It was hypothesised that pathogens were the cause of this population collapse.

In this study, bacteria and viruses present in the invasive ants were investigated using 454 sequencing and Illumina sequencing for future developments of possible biological controls for the Argentine ants, and a better understanding of the observed population decline in New Zealand. Bacterial diversity present in Argentine ants either declined or diminished along the invasion pathway. At the same time, the invasive ants maintained a 
core of nine bacteria genera, including Lactobacillus and Gluconobacter, two bacterial genera with members known for their beneficial associations with honey bees. The presence of these core bacteria may have participated in the success of Argentine ants in their new environments. In the laboratory, the use of ampicillin and gentamicin antibiotics on the ants increased bacterial diversity present in the ants. Furthermore, ampicillin, kanamycin and spectinomycin antibiotic treatments increased ant survival but did not affect the ant fitness or intra-species aggressiveness. Only spectinomycin treated ants presented a higher level of inter-species aggressiveness. Bacterial diversity may play an important role in the ant health and at length population dynamics.

Finally, Argentine ants are the hosts of two viruses: the Deformed wing virus (DWV) involved in colony collapse disorder in honey bees, and Linepithema humile virus 1 (LHUV-1), a new virus related to DWV. Both viruses actively replicate within the ants, indicating a possible reservoir role of the ants. However, the effects of the viruses on the ants are not yet known. Further viral infection in the laboratory under different stress conditions and / or antibiotic treatment will give an insight in the role played by these viruses in the observed population collapse of Argentine ants in New Zealand. LHUV-1 may offer a possibility in the development of the first biological control for Argentine ants, depending on its specificity and its effects.

This dissertation provides a first insight in the core bacteria as well as potential harmful viruses present in Argentine ants. These bacteria and viruses may play a role in the ant population dynamics. Invasive species may co-introduce harmful pathogens with them, and participate to the spread of local ones. The pathogens may affect both invasive ants and native species population dynamics. 


\section{Acknowledgements}

Foremost, I want to deeply thank Phil Lester for giving me the opportunity to do this research, for supporting me through this difficult process, and for never giving up on me.

Thank you to Victoria University for all the supporting funding that allowed me to focus myself solely on my research. And also, thank you to Monica Gruber and Peter Ritchie for their constructive comments on my experiments.

I want to acknowledge the incommensurable help of all the people that surrounded me during these years: Habteab Habtom for being my first guide in Argentine ant collecting and Antoine for these additional samples that improved my virus chapter; Arindam Bhakta, Anna Friedlander, Marc Loupias and Catarina Silva for their computing support; Alistair Brown and David Ackerley's group for all the antibiotics and help on the matter; Kosala Sirisena for sharing his knowledge on 454 sequencing; Adrian Bibby for his help in the laboratory; James Matthews and David Maas's group for giving me insights on bacteria and laboratory equipment; Sebastian Fernandez and Christian Boedeker for their genetic knowledge; Rafael Barbieri for his understanding of $\mathrm{R}$, his patience, his time and his help in the field and in the laboratory; Richard Hall, Jing Wang and Nicole Moore of the Institute of Environmental Science and Research (ESR) for all they have done for the virus hunt, for their time, their support and their patience; Wlodeck Stanislawek for his help with primers, his patience and his major role in our virus discovery; Michele Fontana, Shalen Kumar, and Meghan Cooling for supporting me during the writing process and for being amazing support in my personal life. All these people have helped me both on an academic and on a personal level, and for this I will be forever grateful. 
Merci à mon père, Claude Sébastien. Merci pour ton support constant et sans limite, merci d'avoir été tout au long de ma vie un port d'attache contre les marées, un pilier sans faille et indestructible. Jamais rien de cela n'aurait été possible sans toi. Merci de tout mon cœur. 


\section{Publications from this thesis}

A. Sébastien, P.J. Lester, R.J. Hall, J. Wang, N.E. Moore \& M.A.M. Gruber, (2015). Invasive ants carry novel viruses in their new range and form reservoirs for a honeybee pathogen. Biology Letters, 11 (9), doi: 10.1098/rsbl.2015.0610.

A. Sébastien, M.A.M. Gruber, R. Barbieri \& P.J. Lester (in preparation). Symbiotic bacterial communities in ants are modified by invasion pathway bottlenecks and alter host behaviour. 


\section{Contents}

Chapter 1. General introduction ..................................................................... 19

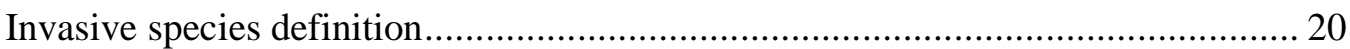

Negative impacts of invasive species in their new environments......................... 20

Invasive species success and the enemy release hypothesis.............................. 22

Infections spillover and spillback between invasive and native species .................. 24

Invasive population decline associated with pathogenic infections and low genetic

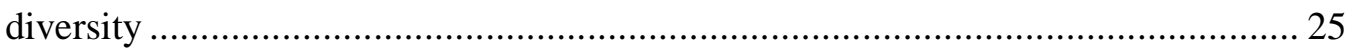

Bio-control definition, applications and limitations...................................... 26

Next-generation sequencing to discover infecting bacteria and viruses ................. 28

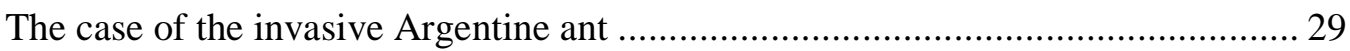

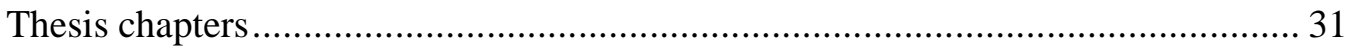

Chapter 2. Bacterial diversity in Argentine ants decline along the ant invasion pathway $\quad$............................................................................................................................. 35

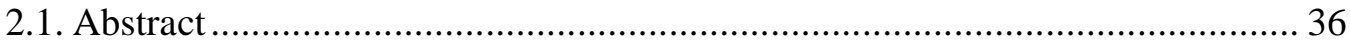

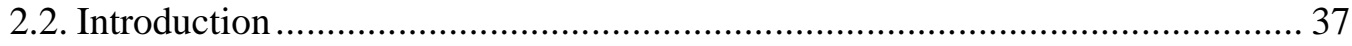

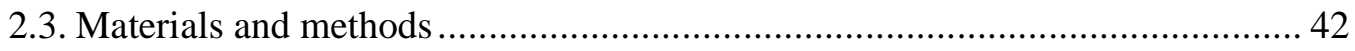

2.3.1. Metagenomics sequencing ......................................................... 43

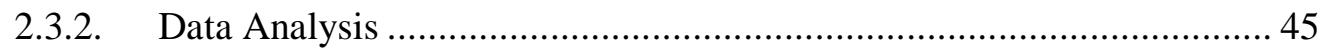

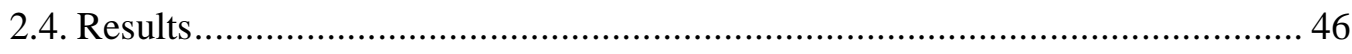

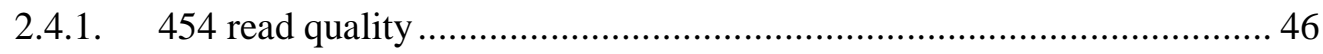


2.4.2. Bacterial diversity among and between countries............................ 47

2.4.3. Bacterial diversity within New Zealand .......................................... 57

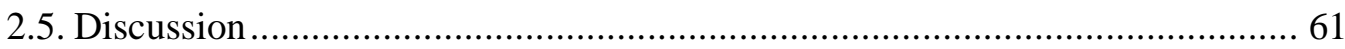

Chapter 3. Bacterial diversity decline in Argentine ants after antibiotic treatments $\quad$............................................................................................................................. 71

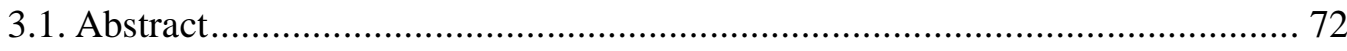

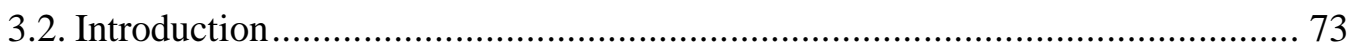

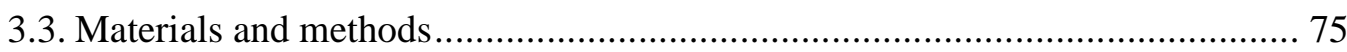

3.3.1. Colony collection and laboratory rearing conditions .......................... 75

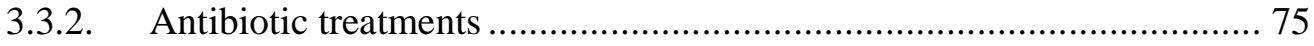

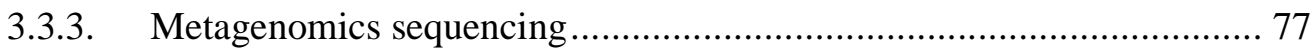

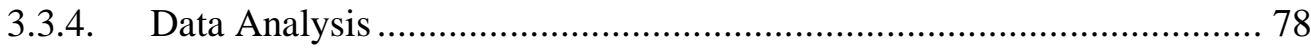

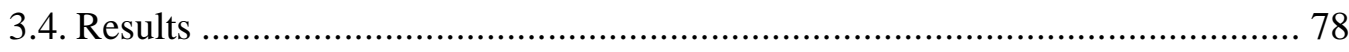

3.4.1. Optimal antibiotic concentration................................................. 78

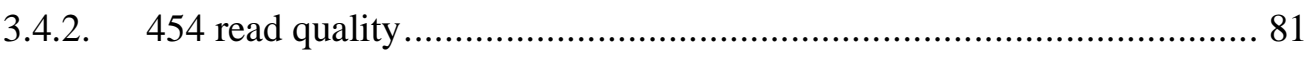

3.4.3. Bacterial diversity between antibiotic treatments ............................ 81

3.4.4. Ant survival and colony fitness after antibiotic treatments ................. 89

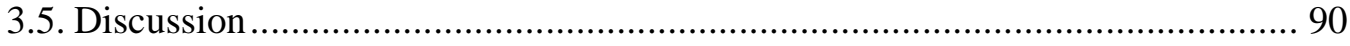

Chapter 4. Argentine ant inter-species aggressiveness increases in the absence of fitness changes after antibiotic treatments .......................................................... 95

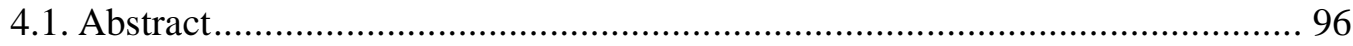

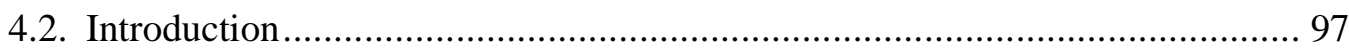

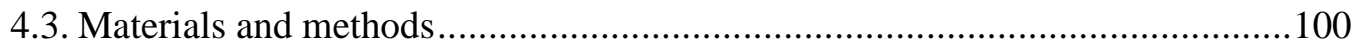

4.3.1. Ant colony collection and laboratory conditions .............................100

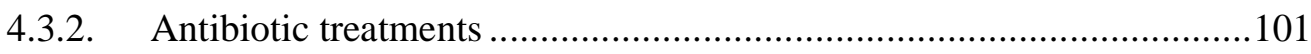

4.3.3. Changes in inter- and intra-specific aggressive interactions ................101

4.3.4. Survey of survival and fitness of colonies......................................103

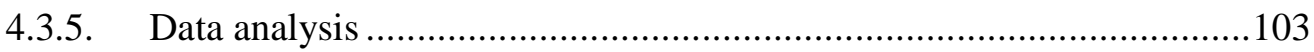


4.4.1. Tests prior to antibiotic treatments ................................................ 103

4.4.2. Argentine ant survival in absence of encounters during antibiotic treatments 106

4.4.3. Argentine ant intra-species aggressiveness and survival during and after antibiotic treatments 108

4.4.4. Argentine ant inter-species aggressiveness and survival during and after antibiotic treatments

4.4.5. Argentine ant survival and fitness after antibiotic treatments

4.5. Discussion 116

Chapter 5. Virus discovery in the invasive Argentine ants

5.1. Abstract 122

5.2. Introduction 123

5.3. Materials and methods 125

5.3.1. Metagenomic discovery of viruses 125

5.3.2. Distribution of Argentine ant viruses within New Zealand and Australia 128

5.4. Results

5.4.1. Metagenomics data analysis and confirmation of contig presence ....... 133

5.4.2. Distribution of Deformed wing virus, Linepithema humile virus -1 and three RNA viral sequences........................................................................ 135

5.4.3. Active replication and prevalence of Deformed wing virus and Linepithema humile virus -1

5.5. Discussion

Chapter 6. General discussion

6.1. Presence of bacteria and viruses in Argentine ants 152

6.2. The decrease of bacterial diversity in invasive Argentine ants was associated with a complex invasive pathway and low genetic diversity 152 
6.3. Potential role of bacterial diversity in Argentine ant population dynamics ......153

6.4. Potential role of viral infections in Argentine ant population dynamics...........154

6.5. Study contributions, study improvements and future directions......................156

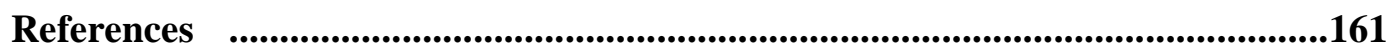

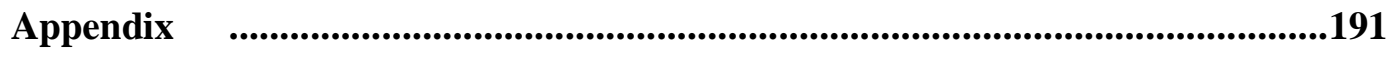

I. Chapter 2: additional materials and methods - sample sites ..........................192

II. Chapter 2: additional materials and methods - density measurements.............194

III. Chapter 4: additional materials and methods - additional antibiotic................195

IV. Chapter 5: additional materials and methods - collection sites......................196

V. Chapter 5: additional results - RNA Illumina sequencing results...................197 


\section{List of figures}

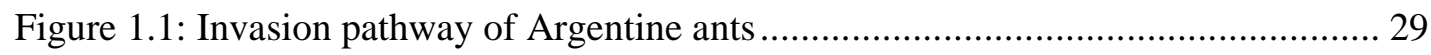

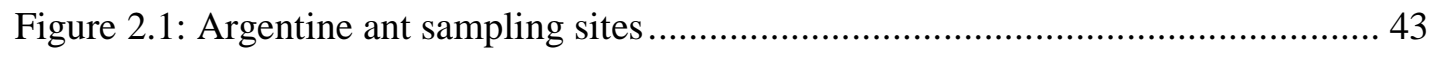

Figure 2.2: Rarefaction curves using QIIME 1.7.0, based on the estimated number of OTUs in Argentine ants in the ant home range and three invaded countries based on the number

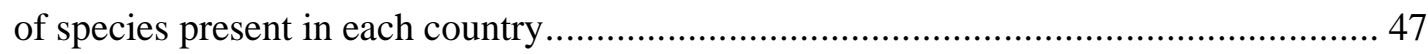

Figure 2.3: Principal coordinate analysis (PCoA) based on a) unweighted and b) weighted phylogenetic UniFrac distances generated in QIIME 1.7.0 between Argentine ant samples

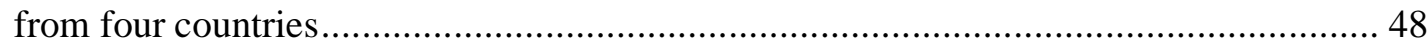

Figure 2.4: Venn diagrams of unique and shared bacteria present in Argentine ants in the ant home range and three invaded countries: a) at the phylum level, and b) at the genus level 49

Figure 2.5: Relative abundance of non-rare bacteria genera in Argentine ants in the ant home range and three invaded countries 54

Figure 2.6: Bacterial diversity at the genus level present in each Argentine ant sampling site in the Argentine ant home range and three invaded countries 55

Figure 2.7: Rarefaction curves using QIIME 1.7.0, based on the estimated number of observed OTUs in Argentine ants in New Zealand between 2011 and 2012 ................. 57

Figure 2.8: Principal coordinate analysis ( $\mathrm{PCoA}$ ) based on a) unweighted and b) weighted phylogenetic UniFrac distances generated in QIIME 1.7.0 between Argentine ants from five cities in New Zealand. 59

Figure 2.9: Venn diagrams of unique and shared bacteria present in Argentine ants in five cities in New Zealand: a) at the phylum level, and b) at the genus level

Figure 3.1: Survival probability of Argentine ants during antibiotic treatments with ampicillin, gentamicin, kanamycin and spectinomycin

Figure 3.2: Survival probability of Argentine ants during antibiotic treatments at different concentration for: a) ampicillin; b) gentamicin; c) kanamycin; and d) spectinomycin .... 80

Figure 3.3: Rarefaction curves using QIIME 1.7.0, based on the estimated number of OTUs in Argentine ants before and after antibiotic treatments 
Figure 3.4: Principal coordinate analysis (PCoA) based on a) unweighted and b) weighted phylogenetic UniFrac distances generated in QIIME 1.7.0 of Argentine ant samples before and after antibiotic treatments .83

Figure 3.5: Venn diagrams of unique and shared bacteria present in Argentine before and after antibiotic treatment: a) at the phylum level, and b) at the genus level .83

Figure 3.6: Relative abundance of non-rare bacteria genera before and after antibiotic treatments 85

Figure 3.7: Survival probability of Argentine ants during and after antibiotic treatments .89

Figure 4.1: Survival probability of ants in the absence of aggressive encounter and prior to any antibiotic treatments 104

Figure 4.2: a) Mean aggression scores of Argentine ant encounters between marked and unmarked workers; b) Survival probability of unmarked and marked Argentine ants after encounter with marked Argentine ants from the same colony 105

Figure 4.3: Survival probability of treated Argentine ants in absence of encounters at different times (days) in the antibiotic treatment, for a) control, b) ampicillin, c) kanamycin, and d) spectinomycin 107

Figure 4.4: Mean aggression scores of ants during inter-species encounters between treated Argentine ants (a) control, b) ampicillin, c) kanamycin, and d) spectinomycin) and marked Argentine ants (a'), b'), c'), and d')) 108

Figure 4.5: Survival probability of intra-species encounters between treated Argentine ants (a) control, b) ampicillin, c) kanamycin, and d) spectinomycin) and non-treaded marked Argentine ants (respectively a'), b'), c'), and d')), at three different times during antibiotic treatment 110

Figure 4.6: Mean aggression scores of ants during inter-species encounters between treated Argentine ants (a) control, b) ampicillin, c) kanamycin, and d) spectinomycin) and Prolasius advenus (a'), b'), c'), and d')).... 112

Figure 4.7: Survival probability of inter-species encounters between treated Argentine ants ((a) control, b) ampicillin, c) kanamycin, and d) spectinomycin) and Prolasius advenus (respectively a'), b'), c'), and d')), at three different times during antibiotic treatment..113

Figure 4.8: Argentine ant colony survival probability during antibiotic treatments $\ldots . . .115$

Figure 5.1: Map of the Argentine ant sampling sites ............................................ 128

Figure 5.2: Identity of the RNA sequences found in Argentine ants: (a) Pie chart of the percentage of RNA sequences classified into each domain and viruses; (b). Classification of all the RNA sequences classified as viruses per viral family 133

Figure 5.3: Maximum-likelihood tree of Dicistroviridae including Linepithema humile

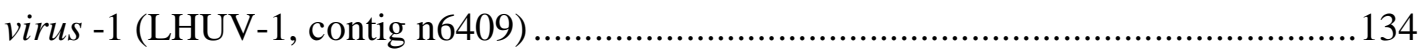


Figure 5.4: Distribution of Deformed wing virus (DWV), Linepithema humile virus -1 (LHUV-1), n1000, n1050 and n1905 in Argentine ant home range (a) Argentina and two invaded countries, (b) Australia and (c) New Zealand..... 135

Figure 5.5: Maximum likelihood tree of Deformed wing virus (DWV) sequences from different countries 136

Figure 5.6: Maximum likelihood tree of Linepithema humile virus -1 (LHUV-1) sequences from different countries 137

Figure 5.7: Maximum likelihood tree of contig n1000 sequences from different countries 138

Figure 5.8: Maximum likelihood tree of contig n1050 sequences from different countries 139

Figure 5.9: Maximum likelihood tree of contig n1905 sequences from different countries 140

Figure 5.10: Infection scenarios occurring at different stage of Argentine ant invasion path for: (a) Linepithema humile virus -1 (LHUV-1) and n1000; (b -1 to -3) n1050 and n1905; and (c -1 to -5) Deformed wing virus (DWV) ........................................................... 147 


\section{List of tables}

Table 2.1: Tagged forward Gray28F 16S universal bacteria primer sequences ............... 44

Table 2.2: Bacteria phyla in the Argentine ant home range, Argentina, and three invaded countries, the United States, Australia and New Zealand .......................................... 50

Table 2.3: Bacteria genera in Argentine ant home range and three invaded countries..... 52

Table 2.4: Relative abundance of bacteria genera present in Argentine ants in all cities 56

Table 2.5: Bacteria phyla present in Argentine ants in New Zealand........................... 59

Table 2.6: Bacteria genera present in Argentine ants in New Zealand ........................... 61

Table 3.1: Bacteria phyla in the Argentine ant before and after antibiotic treatments ..... 84

Table 3.2: Bacteria genera present in Argentine ants before and after antibiotic treatments

86

Table 3.3: Results of the Generalised linear model (GLM) on colony fitness (number of brood) and the significance of the antibiotic treatment, the number of bacteria, workers and

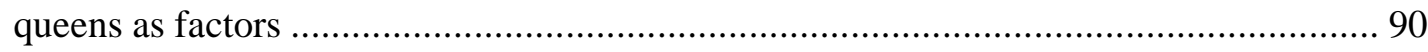

Table 4.1: P-values results of the cox survival analysis in the absence of aggressive

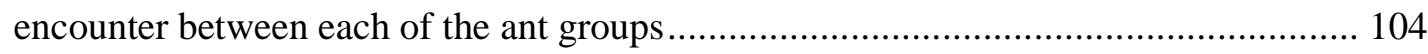

Table 4.2: Likelihood ratio test (coxph) comparison analysis results of survival probability in absence of encounters during antibiotic treatments of Argentine ants...................... 107

Table 4.3: Anova results testing the difference of intra-species aggressiveness of Argentine ants through time for each antibiotic treatment

Table 4.4: Likelihood ratio test (coxph) comparison analysis results of survival probability during intra-species encounters, during antibiotic treatments of Argentine ants.

Table 4.5: Anova results testing the difference of inter-species aggressiveness of Argentine ants through time, for each antibiotic treatment

Table 4.6: Likelihood ratio test (coxph) comparison analysis results of survival probability during inter-species encounters, during antibiotic treatments of Argentine ants............ 114

Table 4.7: Anova analysis results for the difference in fitness (brood production) between antibiotic treatments 
Table 5.1: List of designed primers specific to selected RNA potential viral contig sequences found in Argentine ant after preliminary Illumina sequencing ....................127

Table 5.2: Published primers used to assess the presence / absence Israeli acute paralysis virus (IAPV), Kashmir bee virus (KBV), Acute bee paralysis virus (ABPV), Solenopsis invicta virus -1 and -2 (respectively SINV-1 and SINV-2), and Deformed wing virus (DWV).....

Table 5.3: Tagged primers used to amplified specifically the RNA negative strand of Deformed wing virus (DWV) and Linepithema humile virus-1 (LHUV-1) using a modified one step RT-PCR protocol 131

Table 5.4: Summary table of the presence/absence of viruses of interest in Argentina, Australia and New Zealand 141

Table I.1: Argentine ant sample collections and ant pool composition for $16 \mathrm{~S}$ analysis 181 Table IV.1: Collection sites to map the range of viruses of interest in Argentine ants ... 185 Table V.1: BLASTx similarity statistics for RNA contigs matching viruses in Argentine ant sample in New Zealand with the best hit viruses. 186 
Chapter 1. General introduction 


\section{Invasive species definition}

Invasive species are a global issue. Invasive species are non-native species, "alien species whose introduction does or is likely to cause economic or environmental harm or harm to human health" (ISAC, 2006; Simberloff et al., 2013). A wide range of species from all kingdoms are already classified as invasive species: algae, plants, fishes, mammals and insects among others. These invasive species eliciting their effects on extremely diverse environments, from seas, lakes, forests and mountains (Seipel et al., 2012; Edelist et al., 2013; Cassone et al., 2014; Lee et al., 2014; Shackleton et al., 2014; Harper and Bunbury, 2015).

Human activity is a major cause of species movement (Hulme, 2015; Mihalca, 2015). Species are involuntary and repetitively transported into new environments via human trade, such as shipping or freight industries. Ports and airports are hot spots for invasive species entrances. Only the introduced species that settle successfully into their new environments at the detriment of native species are considered invasive species (Lockwood et al., 2005). Multiple biotic and abiotic factors affect invasive species during their settlement process: (i) propagule pressure, meaning the number of introduced individuals (Lockwood et al., 2005), (ii) climate (Gunawardana et al., 2013), and (iii) species traits, including reproduction characteristics (Rathé et al., 2015). For example, invasive insects such as the Asian tiger mosquito (Aedes albopictus) were transported across continents into Europe both as mated insect females, ready to lay fertile eggs, and as eggs laid on imported goods (Medlock et al., 2012). Under these conditions, a high number of viable individuals are insured to be successfully introduced into new environments, and subsequently facilitated the invasive species settlement.

\section{Negative impacts of invasive species in their new environments}

Once introduced into new environments, invasive species can displace native species and decrease species diversity via multiple mechanisms. Invasive species can dominate native species via direct predation. For example, the consumption of native tree weta (Hemideina thoracica) by the invasive rat (Rattus rattus) population in New Zealand lead to the decline in the weta population (Ruscoe et al., 2013). Invasive species can also compete with native species by monopolising and denying access to food resources (Vonshak et al., 2011). If 
the food source comes from flowers, invasive species may disrupt food collection by native species foragers and indirectly reduce pollination. At length, plant reproduction success can decrease. In Hawaii, invasive western wasps (Vespula pensylvanica) preyed on other pollinators and dominated pollen sources on native trees (Metrosideros polymorpha) (Hanna et al., 2014). As a consequence, native pollinator species populations, including native bees (Hylaeus spp.), were displaced and plant seedling success decreased (Hanna et al., 2014).

Invasive species can also facilitate the spread of other invasive species, further affecting native species diversity (Wood et al., 2015). In New Zealand, the red deer (Cervus elaphus) and the brushtail possum (Trichosurus vulpecula) are invasive species. The two mammal species promote the dispersion of multiple invasive pathogenic fungi (from the genera Rhizopogon and Suillus) via their faeces. The fungi in turn inhibit the growth of infected native beech tree seeds (Lophozonia menziesii) but not of invasive pines (Pinus contorta and Pseudotsuga menziesii) (Wood et al., 2015). Another example is the yellow crazy ant (Anoplolepis gracilipes) invasion in Christmas Islands. The yellow crazy ants have decimated the red crab (Gecarcoidea natalis) population, which are a keystone predator on the island. In addition, the invasive ants cooperated with diverse invasive scale insects for their honeydew. The combined effects of the disappearance of a key predator and the mutualistic association of two invasive species lead at length to the death of canopy trees (O’Dowd et al., 2003).

A lesser known mechanism are disease-mediated invasions when invasive species cointroduce harmful micro-organisms with them (Dunn and Hatcher, 2015; Vilcinskas, 2015). While infections caused by co-introduced micro-organisms may have little to no impact on the host invasive species, they may be fatal to the naïve native species. Cointroduced micro-organisms may subsequently induce the decline of these naïve populations (Arbetman et al., 2013). In Europe, the invasive ladybird (Harmonia axyridis) is the host to multiple parasitic microsporidia. Some of these parasitic microsporidia kill native ladybirds (Coccinella septempunctata) but not invasive ladybirds, whilst facilitating at length the invasion by the invader (Vilcinskas et al., 2013). In the case of the invasive glassy-winged sharpshooter (Homalodisca vitripennis), the plant pathogen carried by the insect (Xylella fastidiosa) causes damages to crops and native plants (Rathé et al., 2012). 
In addition to modifying novel environment species diversity, invasive species can directly impact on countries' economy by affecting human health and agriculture. Some invasive species such as the red imported fire ants (Solenopsis invicta) can attack humans with painful bites and stings, causing allergic reactions needing medical care (Potiwat and Sitcharungsi, 2015). Interactions with some invasive species can even lead to life threatening allergic reactions as it is the case for invasive wasps species from the Vespidae family (Beggs et al., 2011). Furthermore, invasive species can also transmit local or introduced pathogens to humans (Medlock et al., 2012; Montarsi et al., 2015; Ngoagouni et al., 2015). Invasive mosquitos (including Aedes albopictus) in Europe increased the number of vectors transmitting local pathogens such as the dengue fever as well as the introduced chikungunya fever (Medlock et al., 2012). In addition, invasive species can also cause major crop losses by feeding on crops (Walsh et al., 2011) and / or spreading plant pathogens (Shi et al., 2014). The invasive whitefly (Bemisia tabaci) can infect plants with the tomato leaf curl virus (Shi et al., 2014). The virus lowers the plant defence and promotes the plant as a food source for other whiteflies.

Multiple management programs have been developed to limit the negative impacts caused by invasive species on their novel environment species diversity, on human health, and agriculture. Damage caused by invasive species and the cost of management programs are estimated to reach up to billions of dollars to governments (Pimentel et al., 2005; Duffy et al., 2013; Lee et al., 2015). Over 50,000 species in the United States are invasive and the cost of their damage and losses has been estimated to reach US\$ 120 billion per year (Pimentel et al., 2005).

\section{Invasive species success and the enemy release hypothesis}

Invasive species success in their novel environments is thought to be in part due to the enemy release hypothesis (Jeschke, 2014; Prior et al., 2015). The enemy release hypothesis proposes that invasive species escape from their natural enemies, predators and pathogens, and that this escape promotes invasion success. However, few studies associate the enemy release to invasive species success by relating the loss of enemies to population increase (Roy et al., 2011). Red imported fire ants (Solenopsis invicta) host fewer pathogens in their invasive range in the United States than in their home range in Argentina (Yang et al., 
2010). Other factors were hypothesised by the authors to have also contributed to the success of these invasive ants.

During invasion process, invasive species can lose not only predators but also favourable associations such as beneficial facultative micro-organisms. The loss of microbiota especially the midgut microbiota can lower host immunity (Jupatanakul et al., 2014). Asian tiger mosquitoes present a lower bacterial microbiota in their invasive range in France than in their home range in Vietnam (Minard et al., 2015). Only a fraction of the microbiota was common to both countries. This microbiota difference may affect at length the transmission of viruses from mosquitos to human.

Some specific bacteria, such as the facultative endosymbiont genera Wolbachia, Spiroplasma and Hamitonella, are known for their beneficial proprieties to their insect hosts (Chrostek et al., 2013; Hamilton and Perlman, 2013; Su et al., 2013; Asplen et al., 2014; Maistrenko et al., 2015). In Drosophila flies and Bemisia tabaci whiteflies, the bacteria offer protection against multiple viral, bacterial and fungal infections, as well as against nematodes and parasitoids. In addition, they can increase their host life. Another example of a bacteria genus that has frequently been thought to be beneficial is Lactobacillus. Lactobacillus genus includes symbiont species present in the gut of honey bees (Apis mellifera), introduced worldwide for honey production and crop pollination (vanEngelsdorp and Meixner, 2010). This symbiont species are active agents of their bee host immune defence against pathogenic infections (Vásquez et al., 2012; Yoshiyama et al., 2013). The loss of these beneficial associates may reduce invasive species immune systems and leave them more susceptible to pathogenic infections.

While the enemy release hypothesis states that invasive species will present fewer natural enemies in their novel environments (Arundell et al., 2014; Prior et al., 2015), invasive species can still co-introduce micro-organisms with them (Arbetman et al., 2013; Hedtke et al., 2015; Lester et al., 2015b). The co-introduced micro-organisms can be either beneficial or harmful for the hosts. The co-introduction of harmful micro-organisms can be at the detriment of the invasive species. The red imported fire ants present less pathogens in its invaded range than in its home range as previously discussed. However, the invasive red imported fire ants co-introduced from their home range harmful viruses such as Solenopsis invicta viruses -1 and -2 (Yang et al., 2010). Infection by these viruses seem not to have slowed down the red imported fire ant range expansion in the United States. 
Infections by these two viruses are in fact commonly asymptomatic for the ants. Virulence seems to be triggered by various stress factors and can lead to the death of entire ant colonies. Micro-organisms co-introduced by invasive species may influence invasive species population dynamic and their spread.

\section{Infections spillover and spillback between invasive and native species}

Pathogens, co-introduced by invasive species or naturally occurring, can be transmitted by spillover and spillback between species. A spillover corresponds to the transmission of infections from one species to another species (Colla et al., 2006). In the case of invasive and native species, infections can occur either from invasive to native species or from native to invasive species. Naïve native species may be more susceptible to the effects of pathogens co-introduced with invasive species than the invasive original hosts. Infected native species may die in high numbers, facilitating at length the spread of invasive species (Strauss et al., 2012; Lymbery et al., 2014; Dunn and Hatcher, 2015). For example, the squirrel virus pox (Squirrel parapoxvirus) was co-introduced by the grey squirrel (Sciurus carolinensis) into the United Kingdom (Strauss et al., 2012). While the invasive host was shown not to be affected by the virus, the infection is fatal for the native squirrels $(S$. vulgaris), and therefore lead to a dramatic decrease of the native squirrel population (Strauss et al., 2012). Honey bees (Apis spp.) and bumble bees (Bombus spp.) are introduced and reared for honey and field pollination. Pathogens co-introduced with these insects can infect native species. Spillovers of co-introduced pathogens have caused the decline of native bee and bumble bee populations (Meeus et al., 2011; Arbetman et al., 2013; Schmid-Hempel et al., 2014; Gamboa et al., 2015). In parallel, invasive species can also suffer negative effects from locally acquired pathogen infections. In their invasive range in North America, the invasive soybean aphids (Aphis glycines) has a lower fecundity when feeding on plants infected by the Bean pod mottle virus (Cassone et al., 2014).

A spillback corresponds to the infection by spillover from a first species to a second species followed by the infection back from the second species to the first species (Kelly et al., 2009). In the case of invasive species, invasive species can become infected by local microorganisms by spillover and become pathogen reservoirs. Pathogens and parasites may not 
always affect invasive species. In this case, invasive species become asymptomatic reservoirs facilitating the spread of pathogens harmful to native populations by spillback (Kelly et al., 2009). Furthermore, increased virulence of some pathogens may be selected by the passage in their novel invasive species intermediates (Ryabov et al., 2014). For example, most strains of the Deformed wing virus are generally asymptomatic in bees. However, more virulent strains of the virus are found in bees when the virus is transmitted through the mite Varroa destructor (Ryabov et al., 2014). Knowledge regarding the microorganisms carried by invasive species can inform on pathogens that can affect both the invasive and the native species populations.

\section{Invasive population decline associated with pathogenic infections and low genetic diversity}

Even successful invasive species populations may suffer dramatic declines (Simberloff and Gibbons, 2004). Invasive ant populations are subject to important population fluctuations. Yellow crazy ants are a successful invasive species that have caused major damage on species diversity in their invasive rage (Hill et al., 2003; O'Dowd et al., 2003). Recently there has been reports of declining populations in Tokelau (Gruber et al., 2012), Australia (Cooling and Hoffmann, 2015) and the Seychelles (Haines and Haines, 1978).

Multiple factors affect invasive population dynamics and cause population decline, such as extreme weather conditions. The invasive African frog (Xenopus laevis) population dramatically declined in the United Kingdom after two successive extremely cold and dry winters (Tinsley et al., 2015). Increased rain fall promoted the infection of the invasive grass (Bromus tectorum) in North America by the native fungal pathogen (Ustilago bullata) (Prevéy and Seastedt, 2015). In turn, the fungus led to the death of the infected invasive grass. Indeed, pathogen infections may cause the decline of invasive populations (Simberloff and Gibbons, 2004). While invasive species can be released from natural enemies, they may still be infected by pathogens in their new environments. Pathogen infection was hypothesised as the cause of the reported population decline of yellow crazy ants in Australia (Cooling and Hoffmann, 2015).

Pathogenic infections in invasive species may be facilitated by the low genetic diversity of invasive species due to the founder effect. The founder effect corresponds to the loss of 
genetic diversity caused by the limited number of individuals at the origin of the invasive populations. Many invasive species have been shown to present a lower genetic diversity in their invasive range than in their home range. Many invasive species are introduced in low numbers during few events in their novel environments at the origin of a founder effect. This situation leads to potential genetic bottlenecks, resulting in a dramatic loss of genetic diversity (Auger-Rozenberg et al., 2012). A small number of paper wasps (Polistes chinensis antennalis) were successfully introduced in New Zealand from Japan during two distinct events (Tsuchida et al., 2014). The founder effect and genetic bottleneck lead to a lower genetic diversity in the wasps in their introduced range than in their home range. Genetic loss in invasive species may be prevented by multiple introductions of a high number of individuals. For example, the Japanese rose (Rosa rugosa) was introduced in high number and multiple times in Europe promoting the invasion of the plant without any genetic diversity loss.

A lower genetic diversity can affect invasive species immunity and resistance to infections (King and Lively, 2012). By presenting a lower heterozygoty of immune genes, invasive species may be more vulnerable to harmful infections. In Europe, the invasive garden ant (Lasius neglectus) presents a higher infection rate by the invasive pathogenic fungus Laboulbenia formicarum in super colonies than in discrete colonies (Tragust et al., 2015). Other invasive ant species may form these super colonies structures that may affect the spread of viruses in the population. Super colonies are large colonies composed of multiples nests interconnected, exchanging queens, workers, brood and food while discrete colonies correspond to small nests with fewer queens and workers (Suhr et al., 2011). Genetic diversity among workers is lower in super colonies than among workers from discrete colonies. The higher pathogenic fungal infection rates found in the invasive garden from super colonies ants indicates the importance of genetic diversity in infection rates.

\section{Bio-control definition, applications and limitations}

Management programs of invasive species can cost billions of dollars. They principally involve the use of poisons, chemicals and toxins (Ward et al., 2010; Shackleton et al., 2014). Chemicals like fipronil are successfully used to control invasive species, such as the invasive western yellowjacket wasp (Vespula pensylvanica) in Hawaii with limited impact on other arthropods (Hanna et al., 2012). Knowledge of the biology of the invasive species 
target for management control can improve the success of the programs. For example, knowledge on the location of the nests, the number of queens and the variation in brood production throughout the year in the yellow crazy ants (Hoffmann, 2014; Hoffmann et al., 2014) can improve the selection of the treatment location and season for the management of these invasive species. The use of poisons, chemicals and toxins are potentially harmful for native and reared species (Barbieri et al., 2013; Di Prisco et al., 2013; Lu et al., 2014; Wagner and Nelson, 2014). Control of feral cats by poison baits in Australia was estimated to potentially affect over $12 \%$ of native species that could consume the baits (Buckmaster et al., 2014). Fungicide use around beehives induce a state of poor nutrition in the bees and a higher level of virus infections (Degrandi-Hoffman et al., 2015).

Biological controls, or bio-controls, are developed in ordered to reduce the negative side effects of pesticides and insecticides on native species, and species of economic value such as honey bees. Bio-controls are to natural enemies of the invasive species of interest: parasitoids, viruses, bacteria, fungi, parasites or even natural predators. Possible bio-control agents for the invasive red imported fire ant came from the ant home range and involved multiple pathogenic fungi, parasitoids and nematodes (Briano et al., 2012). Harmful viruses were also discovered in the invasive ants novel environment (Valles et al., 2004; Tufts et al., 2010). Similarly, pathogenic micro-organisms are investigated to control invasive plants such as Phragmites australis (Kowalski et al., 2015).

The specificity, optimal infection concentration for a pathogen, or density of release of a predator, and the holistic effect of the biological control on the invasive species must be tested prior to their applications as bio-control agents (Porter et al., 2013; Valles and Porter, 2015). Bio-control agents have the potential to become pests or invasive species themselves if not carefully studied (Yamanaka et al., 2015). For example, the ladybird (Harmonia axyridis) was introduced in Europe as a bio-control for aphid populations, and have now become an invasive species (Vilcinskas et al., 2013). In addition, the invasive ladybirds cointroduced an obligate microsporidia (related to Nosema thompsoni). While not harmful to their invasive hosts, the microsporidia are deadly to native ladybirds (Coccinella septempunctata) (Vilcinskas et al., 2013).

Furthermore, the control or complete eradication of one invasive species will not necessary promote the return of native species. The interaction between invasive species and their novel environment is complex. Invasive species can reduce some native species 
populations and facilitate others like the invasive cane toads (Rhinella marina) in Australia (Jolly et al., 2015). Some invasive species interact with other invasive species, promoting each other spread, such as red imported fire ants and mealybugs (Helms and Vinson, 2002). Finally, invasive species can compete among themselves, limiting each other populations. For example, in New Zealand, the control of possums lead to an increase of rats while the control of rats led to an increase of mice (Ruscoe et al., 2011). Only controlling one invasive species released the other species from food competition. Invasive species control and native environment recovery may therefore need complex programs.

\section{Next-generation sequencing to discover infecting bacteria and viruses}

The use of next generation sequencing may offer a first global insight in describing bacteria and viruses present in the invasive species. Firstly, 454 and Illumina sequencing are high throughput sequencing techniques that allow the simultaneous sequencing of genetic material fragments (Mardis, 2008a, 2008b). Both techniques can sequence separately either DNA or RNA, and allow the creation of libraries regrouping "reads", meaning the amplified sequences. The two techniques differ in the length and the number of reads. On one hand, 454 sequencing create libraries of $\sim 100,000$ reads, each read is $\sim 200-600$ nucleotide long. On the other hand, Illumina sequencing create larger libraries containing over a million short reads each, $~ 100$ nucleotide long. Each sequencing may offer different benefits in the discovery of bacteria and viruses present in invasive species.

Both 454 sequencing and Illumina sequencing have been used in order to investigate and characterise bacteria and viruses present in introduced and invasive species (Ishak et al., 2011b; Johansson et al., 2013; Billiet et al., 2015). First, 454 sequencing can be used to specifically analyse bacterial diversity. Because 454 sequencing allows the sequencing of long reads, this technique can be associated with the use of universal primers such as the primers amplifying the $16 \mathrm{~S}$ bacterial gene. The $16 \mathrm{~S}$ primers amplify DNA during PCRs. The PCR products are then cleaned and sequenced using 454 sequencing. It is important to note that when using $16 \mathrm{~S}$ universal primers, the product length is in average of 500 bases. This read length is enough to analyse reads to the genus level but not to the species level. Confirmation of the bacterial species requires longer reads. Secondly, Illumina sequencing 
is especially used for new sequence discovery. Illumina sequencing allows the sequencing of a high number of short reads. The short reads can be associated in contigs, larger sequences of overlapping reads. The contigs can then be analysed, and may identify to the species level depending on their length. The sequencing technique choice between 454 sequencing and Illumina sequencing depends on the study question.

\section{The case of the invasive Argentine ant}

The Argentine ant (Linepithema humile) is native from South America and originally occurred in Argentina, Brazil, Paraguay, and Uruguay (Wetterer et al., 2009). The ants have successfully spread worldwide in Mediterranean like environments (Wetterer et al., 2009) (Figure 1.1).

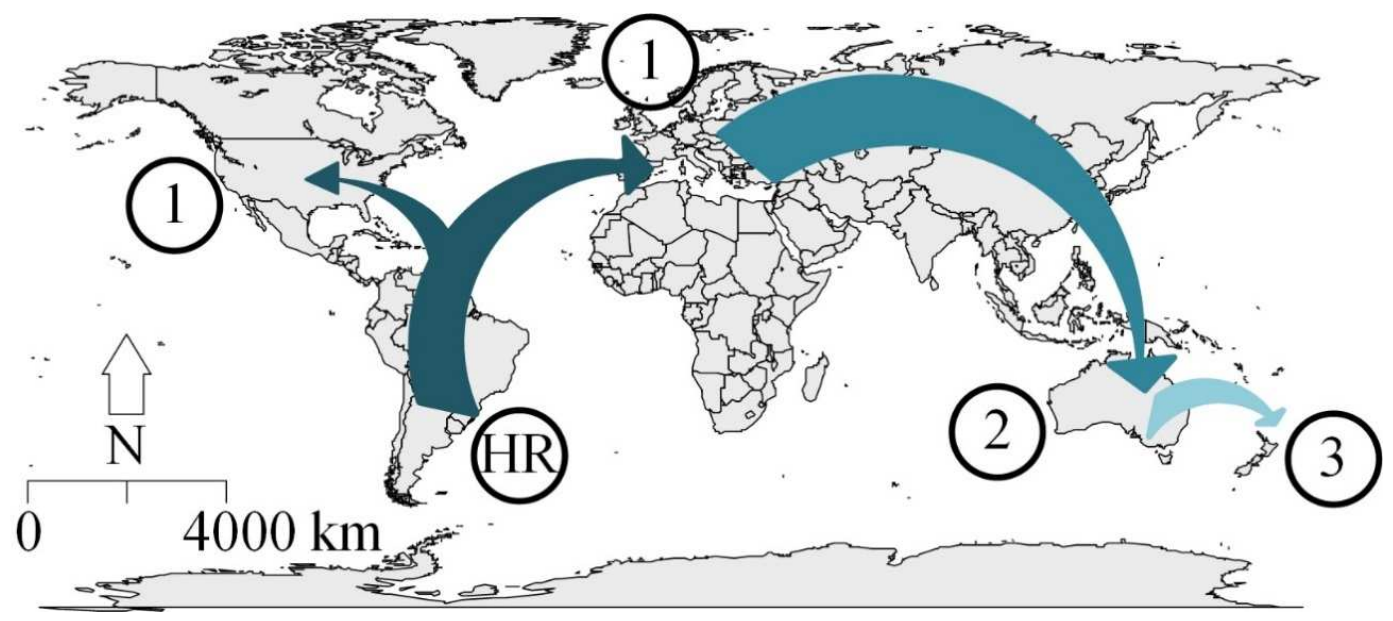

Figure 1.1: Invasion pathway of Argentine ants. Arrows indicate ant population movements from the ant home range in Argentina (HR) to invaded countries. Numbers in the circles represent the invasion type of the countries in question: 1 - primary invasion for the United States and Europe, 2 - secondary invasion for Australia, and 3 - tertiary invasion for New Zealand. Arrows are of decreasing colours. The decrease of colours indicates the decrease of genetic diversity of invasive Argentine ant populations along their invasion pathway.

The spread of these invasive ants occurred during multiple introductory steps facilitated by human activities (Suarez et al., 2001). Argentine ant populations have been introduced from Argentina to the South-East of the United States in 1891 from where they later spread to the rest of the country (Wetterer et al., 2009; Vogel et al., 2010), and different introductions 
from Argentina led to records of the ants in Europe as early as 1858 (Wetterer et al., 2009). The introduction from the home range to a first invaded country is considered a primary invasion. The population in Europe was likely the source of the Australian population. Since the Australian population did not originate from the ant home range but from a primary invaded country, it is therefore considered to be the result of a secondary introduction in 1931 (Wetterer et al., 2009; Suhr et al., 2011). Later, the established population in the east coast of Australia was introduced in New Zealand, and was first observed in 1990 (Corin et al., 2007a, 2007b). The New Zealand population was introduced from a population present in a secondary invaded country, and consequently corresponds to a tertiary introduction.

In their new environments, Argentine ants form large colonies composed of multiple nests, larger than in their home range, with low genetic diversity between them (Suarez et al., 1999). Nests contain multiple queens, and exchange workers, brood and food (Heller et al., 2008). High density in their introduced range allows the ants to promptly recruit from nearby trails, compete and successfully dominate food resources (Human and Gordon, 1996; Holway, 1999; Flanagan et al., 2013; Gordon and Heller, 2014). The invasive ants interfere with the pollination of native plant (Hanna et al., 2015), displace native species, and eventually modify species communities (Human and Gordon, 1996; Sanders et al., 2003). Argentine ants also tend to plant feeding insects, such as scale insects, mealybugs and aphids for their honey dew production, which can at length indirectly inducing possible crop loses (Lester et al., 2003).

In New Zealand, Argentine ants constitute of a well-established single large colony, with low genetic diversity (Corin et al., 2007a). Populations have been present from the top North of the North Island to Christchurch in the South Island (Ward et al., 2010). The invasive ants were reported to displace native species populations, such as arthropods including ants (Holway, 1999), to interfere with native plant pollination (Levan et al., 2014), and to decrease at length biodiversity (Sanders et al., 2003). Management programs in New Zealand are estimated to cost up to NZ\$ 1 million a year (Ward et al., 2010). Standard management programs involve the use of chemicals and pesticides, either as sprays or baits (Ward et al., 2010). To increase Argentine ant specific control, a recent study investigated the use of pheromones (Westermann et al., 2015). 
A large scale Argentine ant population collapse was reported in New Zealand by Cooling et al. (2012). Pathogens were hypothesised to be the probable cause. The large spread of pathogens in the Argentine ant population may be possibly due to potential lower immunity of individuals. Invasive Argentine ants present a low genetic diversity in New Zealand (Corin et al., 2007a) resulting from the multiple successive bottlenecks the population underwent during the invasion process (Suarez et al., 1999). Argentine ants may consequently present a lower immune gene diversity in New Zealand than in their home range. Furthermore, while the multiple invasive bottlenecks may have decreased the number of harmful pathogens, the number of favourable endosymbionts associated with the ants may also have decrease. Therefore the ant associated defences against infections may have also decreased. Finally, the Argentine ant colony structure based on multiple nests and the exchange of food and individuals may facilitate the spread of infections between nests.

No knowledge on favourable associations and pathogenic infections that could affect Argentine ant population is yet available as no previous studies have yet reported the presence of bacteria or viruses in Argentine ants. As a consequence, no bio-control potions for the Argentine ants have to date been developed.

\section{Thesis chapters}

Knowledge on bacteria and viruses present in Argentine ants may offer a first step in the development of a bio-control and a first insight in the ant population collapse in New Zealand. This thesis aimed to: 1) characterise bacterial diversity along the ant invasion pathway; 2) test the effects of change of bacterial diversity on Argentine ant fitness and aggressiveness; and 3) detect for the first time viruses present in Argentine ants. Below is the list of the thesis chapters. For each chapter, the aims and the names of the people involved are indicated.

\section{- Chapter 2: Bacterial diversity decline in Argentine ants}

The aim of this chapter was to characterise the bacterial diversity decline of Argentine ant along their invasion pathway using 454 sequencing. The loss of bacteria was investigated using ant samples from the ant home range, Argentina, and three invaded countries, the 
United States (primary invasion), Australia (secondary invasion), and New Zealand (tertiary invasion). Possible favourable endosymbionts conserved during the ant invasion history are highlighted. This chapter and experimental design were developed with Philip J. Lester and Monica A. M. Gruber.

- Chapter 3: Bacterial diversity decline in Argentine ants after antibiotic treatments

The aim of this chapter was to characterise the change in bacterial diversity in Argentine ants after antibiotic treatments using 454 sequencing technology. Argentine ants were treated with ampicillin and gentamicin before analysing the bacterial diversity present in the ants. These preliminary results will be used in the next chapter investigating the effects of the loss of bacterial diversity on Argentine ant fitness and aggressiveness. This chapter and experimental design were developed with Philip J. Lester, Monica A. M. Gruber, and Rafael Barbieri.

- Chapter 4: Argentine ants inter-species aggressiveness increase in the absence of fitness changes after antibiotic treatments

The aim of this chapter was to test the effects of the decrease of bacterial diversity on Argentine ant fitness and aggressiveness. Argentine ants were treated with ampicillin, kanamycin and spectinomycin. Then the ant fitness and the modification of intra- and interspecies aggressiveness level was observed. This chapter, experimental design and data analyses were developed with Philip J. Lester and Rafael Barbieri.

- Chapter 5: First virus detection in the Argentine ant and infection range in New Zealand

The aim of this chapter was to investigate the presence of viruses in Argentine ants using Illumina sequencing and RT-PCR. This chapter was realised in association with The Institute of Environmental Science and Research (ESR - Wellington, New Zealand). This chapter and experimental design were developed with Philip J. Lester, Richard J. Hall, Jing Wang, Nicole E. Moore from ESR, and Monica A. M. Gruber. Jing Wang was responsible 
for all the Illumina sequencing data analyses. This chapter was published in September 2015 (Sébastien et al., 2015). 
Chapter 2. Bacterial diversity in Argentine ants decline along the ant invasion pathway 


\subsection{Abstract}

Bacterial diversity may promote their host health by participating to their host defences against pathogenic infections. For invasive species, bacterial diversity may decrease along the invasion pathway. Founder effects and bottlenecks may decrease the number of favourable bacteria present in invasive species, which in turn may lower the invasive species defence against pathogenic infections. As some population declines have been associated with pathogenic infects, bacterial diversity may play a role in invasive population dynamics. Originally from South America, Argentine ants (Linepithema humile) are an invasive species in New Zealand. The population in New Zealand was accidentally introduced after a complex invasion pathway. Argentine ants were accidentally introduced from Argentina to Europe, then from Europe to Australia, and finally from Australia to New Zealand. While the population thrived enough to spread in the country, the invasive ant population was reported to have collapsed. Pathogenic infections were suggested as the potential cause. As a first step to understand this population decline, bacterial diversity in Argentine ants was investigated using 454 sequencing technology using samples from the ant home range (four sites in Argentina) and three invaded countries (five sites in the United States, one in Australia and five in New Zealand). Bacterial diversity declined from the ant home range to invaded countries following the ant invasion pathway: bacterial diversity was the highest in the ant home range and in the United States (primary invasion), and the lowest in Australia (secondary invasion) and in New Zealand (tertiary invasion). The ants lost Wolbachia and Rickettsia, two bacterial genera including symbionts known to protect some of their hosts against viral infections, promote their host growth and induce sex biased toward females. However, the ants conserved a core of nine bacterial genera, including Lactobacillus and Gluconobacter, two bacterial genera known include species involved in bee health and protection against some pathogenic infections. The low bacterial diversity in New Zealand associated with the reported low genetic diversity of the ants in the country may have decrease the ant resistance against pathogenic infections. Invasive species may leave natural enemies in their home range but may still be susceptible to new infections. 


\subsection{Introduction}

\section{Beneficial and pathogenic bacteria}

Population dynamics can be affected by bacterial diversity present in the host species. Bacteria can promote their host fitness in mutualistic relationships (Yoshiyama and Kimura, 2009; Koehler and Kaltenpoth, 2013), or at the extreme opposite be fatal if infections are pathogenic (Evans and Schwarz, 2011; Strunov and Kiseleva, 2015). The presence of some bacteria and loss of others may be either beneficial or detrimental to infected populations. In the case of invasive species, bacterial diversity may decrease during the invasion process (Roy et al., 2011). The decrease of bacterial diversity may affect the spread and population dynamics of the invasive species.

Beneficial bacteria can be either obligate or facultative bacteria. Their presence is favourable to the wellbeing of their hosts. Obligate bacteria associations are necessary for the bacteria host survival, such as with the gut bacteria symbionts of the southern green stinkbugs (Nezara viridula) in Japan (Tada et al., 2011). Beneficial bacteria can participate to their host development, nutrition and defence against pathogenic infections. Carpenter ants (Camponotus fellah) carry the obligate endosymbiotic bacterium Blochmannia. Blochmannia improves the ant colony growth and the ant defence against pathogenic infections (de Souza et al., 2009). The obligate endosymbiont Portiera of whiteflies (Bemisia tabaci) provides its hosts with carotenoids, which appear to prevent DNA damage and provide genomic stability (Sloan and Moran, 2012).

Facultative bacteria can also promote their host health by participating in their defence against pathogenic infections and by providing their hosts with complementary nutriments. Some bacteria such as Burkholderia sp. and the bacteria composing the bio-film on leafcutting ants (Atta sexdens rubropilosa and Acromyrmex subterraneus subterraneus) produce antibiotic and antifungal compounds that support their host immune defences (Santos et al., 2004; Mattoso et al., 2012). Similarly, Streptomyces bacteria produce antibiotic compounds that provide some protection to their beewolf hosts (Philanthus triangulum) against pathogenic infections during hibernation (Koehler et al., 2013). Lactobacillus and Bacillus bacterial genera are important genera that include bacterial species associated with the health of their bee hosts (Apis spp.). The bacteria can inhibit 
infections via the productions of antibiotic compounds (Forsgren et al., 2009; Yoshiyama and Kimura, 2009; Vásquez et al., 2012).

Other facultative bacteria have multiple complex effects on their hosts. Wolbachia and Rickettsia are two facultative endosymbionts that can promote their host fitness and survival. Wolbachia protects its fly hosts (Drosophila spp.) against multiple RNA viral infections and increases its host lifespan (Teixeira et al., 2008; Martinez et al., 2014). Rickettsia for its part increases its whitefly hosts (Bemisia tabaci) fecundity, speeds its hosts development, and protects its host against some bacterial infections (Asiimwe et al., 2014; Hendry et al., 2014). However, both bacteria can also induce pathogenicity, cytoplasmic incompatibility and can promote female over male production (Hagimori et al., 2006; Blagrove et al., 2012; Asiimwe et al., 2014). The presence of beneficial bacteria can consequently promote the population growth of their hosts. In parallel, the loss of beneficial bacteria may be detrimental enough to induce a population decline.

In contrast to favourable bacterial species, pathogenic bacteria may induce population declines by killing their hosts. The pathogenicity of a bacteria corresponds to the negative effects of the bacteria on their hosts. For example, the American foulbrood agent is a highly infectious bacterium (Paenibacillus larvae) that infects and kills specifically bee larvae (Apis spp.) (Genersch, 2010a). Infected bee colonies are weakened and more susceptible to predation (Genersch, 2010a). Multiple bacteria can also damage and kill plants (Mansfield et al., 2012). Among these pathogenic bacteria, Erwinia amylovora, Pseudomonas syringae and Ralstonia solanacearum outbreaks can lead to a high number of plants. Pathogenic bacteria may induce a negative pressure on the growth of infected population.

\section{Diversity of bacteria associated with host health}

Single bacteria species can have important direct effects on their hosts, either by promoting their host fitness or by causing its host death. As bacteria can also interact with other bacteria (Yoshiyama and Kimura, 2009), bacterial diversity and not only the presence of a single species has been associated with the health and wellbeing of their hosts (Koch and Schmid-Hempel, 2011; Poulsen and Sapountzis, 2012; Vásquez et al., 2012). In flies (Drosophila spp.), gut bacteria are sufficient to prevent the pathogenic bacteria Gluconobacter morbifer from multiplying in their hosts (Crotti et al., 2010). Later, in aging flies, the change of gut bacterial diversity leads to the modification of the proprieties of the 
fly intestine and the modification of immune gene activation, which eventually leads to the death of the aging flies (Clark et al., 2015).

While the bacterial diversity present in an organism varies over time, some bacteria are consistently present in these organisms. These bacteria correspond to core bacteria and are important for the host health (Anderson et al., 2013). Bumble bees (Bumbus spp.) are less likely to be infected by gut parasite (Crithidia) when their core bacteria Gilliamella are abundant (Cariveau et al., 2014).

\section{Bacteria transmission}

Bacteria can be acquired following two different non-exclusive paths: vertical and horizontal transmissions. Vertical transmission is the transmission of bacteria from parents to offspring (Chen et al., 2006; Evans and Schwarz, 2011). The bacterium Cardium is maternally transmitted in parasitic wasps (Encarsia pergandiella) in which the bacterium induces cytoplasmic incompatibility and a reduction in fertility (Perlman et al., 2008). In the pea aphids (Acyrthosiphon pisum), males and females can transmit beneficial endosymbionts to their offspring that confer protection against parasitoids, such as the bacterium Hamiltonella defensa (Smith and Mueller, 2015).

Horizontal transmission is the transmission of bacterial infection from one individual to another individual, neither of the individuals being the parent or the offspring of the other (Chen et al., 2006; Evans and Schwarz, 2011). The transmission can occur by direct contact between individuals. For social insects, it may occur during the exchange of food by trophallaxis between workers. Transmission between individuals is also possible during allo-grooming, when an individual cleans an infected individual (Evans and Schwarz, 2011). Direct contact may also occur through predation and the ingestion of infected prey transferring bacterial infection from one species to another.

Horizontal transmission may also occur indirectly between individuals, with no direct contact. In bumble bees (Bumbus terrestris), young workers are in contact with adult worker faeces just after eclosion. This contact allows the transmission of necessary gut bacteria from adult workers to young callows (Koch and Schmid-Hempel, 2011). Gut bacteria in bumble bees protect their hosts against infections by parasites such as Crithidia bombi (Koch and Schmid-Hempel, 2011). Another path is the contamination of a food 
source such as pollen on a flower by infected individuals. Later a different individual may forage on the contaminated food source and may become infected itself.

Some bacteria can be transmitted via multiple paths, vertical and horizontal transmission, direct and indirect contact. Wolbachia for example can be transmitted from one individual to another through both vertical and horizontal transmissions (Zug and Hammerstein, 2015). In the leaf-cutting ants (Acromyrmex octospinosus), the beneficial bacteria Streptomyces is transmitted horizontally directly from older workers to callows (Poulsen et al., 2003). The transfer of the beneficial bacteria to new workers may also occur indirectly via the ant fungus garden. Individuals may therefore harbour a diversity of bacteria, some of them inherited by their parents and some acquired from their environment, by contact with individuals from the same species or different ones.

\section{Bacterial diversity decrease during the invasion process}

In invasive species, genetic diversity is likely to decline with each consecutive invasion event steps, because of successive bottleneck events. Consequently, genetic diversity should be lower in an invaded country after a secondary / tertiary invasion then after a primary invasion. The invasive potato tuber moth (Tecia solanivora) populations in South America originated from one to few introductions (Puillandre et al., 2008). In the case of the population in the Canary Island, the moths were first introduced from their hypothetical home range Guatemala to Venezuela, and then from Venezuela to the Canary Islands. These invasion events caused genetic bottlenecks leading to a reduced genetic diversity in the moths. Moth genetic diversity decreases from their home range to the first invaded country, Venezuela, and is the lowest in the second invaded country, the Canary Islands.

Furthermore, genetic diversity of individuals is associated with their health (Mattila et al., 2012). A high genetic diversity is associated with a high diversity of immune genes, and consequently to a better immune defence (Schlüns and Crozier, 2009). In addition, genetic diversity of individuals also influences bacteria associated with them. In honey bees (Apis mellifera), genetically diverse colonies present more active beneficial bacteria and less pathogenic bacteria than colonies with a lower genetic diversity (Mattila et al., 2012). The loss of genetic diversity may affect both the innate and associated immunity of invasive species. 
In addition to genetic bottlenecks, the founder effect may also cause bottlenecks on of the diversity of bacteria associated with invasive species (Minard et al., 2015). Similarly to the genetic diversity decreasing with each invasive step needed to arrive into a new country, bacterial diversity should decrease with each additional invasive step. As bacterial diversity has been associated with their host health and needs during the development (Cariveau et al., 2014; Coon et al., 2014), a decrease in bacterial diversity in invasive species may affect their health and consequently their population dynamics as well.

Both detrimental and favourable associations can be lost during the invasion process (Yang et al., 2010; Roy et al., 2011; Rey et al., 2013; Iwase et al., 2015). According to the enemy release hypothesis, invasive species may lose a large number of their natural enemies, including pathogens, during the invasion process (Yang et al., 2010; Roy et al., 2011). The introduced individuals may not have carried the associated bacteria which may thus be absent from the introduced population. It is also possible that a few introduced individuals were infected but these individuals did not survive the invasion process. This loss of natural enemies has been hypothesised to contribute to invasive species success. Consequently, the diversity of harmful pathogens should be lower in their new environment than in their home range. Invasive red imported fire ants (Solenopsis invicta) present less pathogenic infections in their introduced range than in their home range (Yang et al., 2010). Alternatively, the loss of favourable associations may be detrimental to the spread of the invasive species.

Invasive species may acquire new bacteria in their new environments by horizontal transfer. The alfalfa weevils (Hypera postica) lost their endosymbiont Wolbachia during their introduction in Japan (Iwase et al., 2015). Later, the invasive weevils were infected by the local Wolbachia strain that differs from the weevil home range strain. Furthermore, invasive species will accumulate new pathogens proportionally to the time spent in their new environment and to the diversity present in that environment (Flory and Clay, 2013). Invasive species may present different bacteria between their home range and invaded countries.

\section{The case of the invasive Argentine ant}

As described in Chapter 1 (section 1.8), the Argentine ant (Linepithema humile) is an invasive species in New Zealand. The ants was introduced after multiple successive 
invasion steps from its home range Argentina to New Zealand (see General Introduction, section 1.8). Therefore, this invasive ant and its associated pathogens and endosymbionts underwent multiple bottlenecks through their invasion history. These invasion events may have led to the decrease of a diversity of bacteria, both pathogenic and favourable. The decrease of bacterial diversity should reflect the ant invasion pathway, decreasing from the home range to the first, secondary and finally tertiary invaded countries. The decrease in bacterial diversity may affect the ants. By losing favourable bacteria associations, the ants may have a lower defence against pathogenic infections. However, losing pathogenic bacteria enemies may promote the spread of the ant population. It is also possible that the ants acquired new bacterial infections by horizontal transfer in their new range. Argentine ants may present some infections specific to each invaded country.

\section{Chapter aims}

Using 454 sequencing technology, the bacterial diversity decrease of Argentine ants was characterised along their invasion path, from their home range Argentina to three invaded countries namely the United States, Australia and New Zealand. It was hypothesised that: 1) bacterial diversity present in Argentine ants is higher in the ants home range than in invaded countries; 2) bacterial diversity reflects the number of invasion steps, and consequently decreases in order from Argentina, to the primary invaded The United States, then the secondary invaded Australia and finally the tertiary invaded New Zealand; 3) the bacterial diversity in Argentine ants is similar throughout New Zealand; and 4) a set of core bacteria necessary to Argentine ants is present in ants regardless of invasion history.

\subsection{Materials and methods}

Argentine ant workers were collected between 1999 and 2012 in their home range Argentina (in four cities / area) and in three invaded countries, the United States, Australia and New Zealand (in respectively four, one and five cities / area) (Figure 2.1; see Appendix I). Ants were stored in $70-100 \%$ ethanol at $-20^{\circ} \mathrm{C}$. 


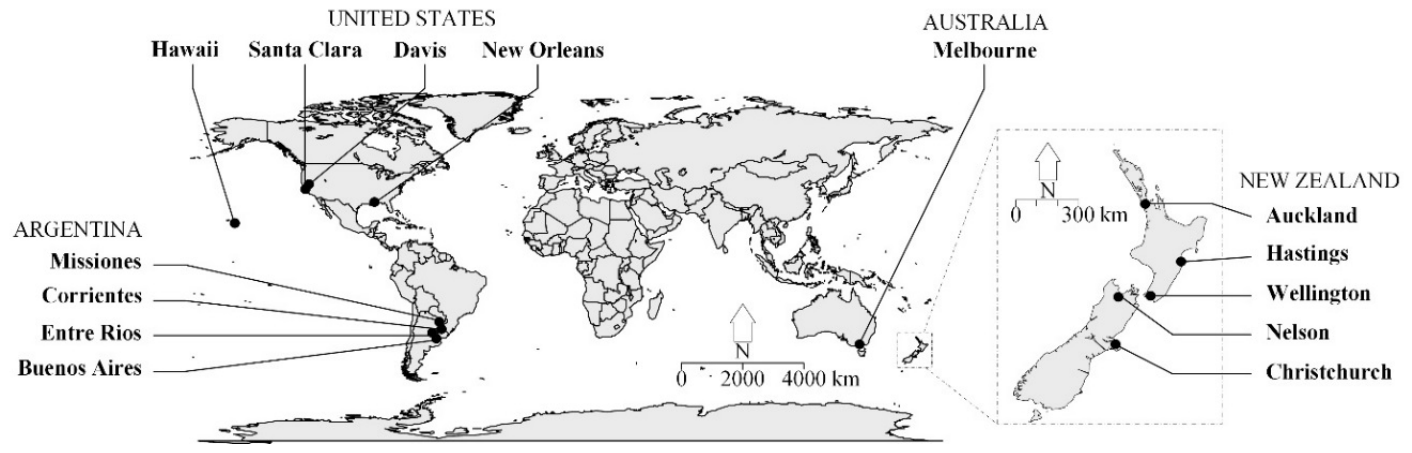

Figure 2.1: Argentine ant sampling sites. Argentine ant samples from Argentina and from the United States, but Davis site, were collected between 1999 and 2002. Samples from the United States site Davis, Australia and New Zealand were collected between 2011 and 2012.

In order to separate samples in New Zealand between high and low density sites, Argentine ant densities were measured using honey baits. Honey bait measures were chose in order to compare the results with a previous density assessment of Argentine ant density in New Zealand (Cooling et al., 2012). However, results indicated that the density measurements were not reliable, and therefore were not further considered (see Appendix II).

\subsubsection{Metagenomics sequencing}

\section{DNA extraction}

To retrieve bacterial DNA from the samples, DNA was extracted from a pool of 30 ants from each sampling site (see Table 1.1). DNA was extracted using a PureLink® Genomic DNA Mini-Kit (Invitrogen) following the manufacturer's protocol. The extractions were further purified using the standard Phenol-Chloroform protocol. In detail, each sample was placed in a $1.7 \mu \mathrm{L}$ microcentrifuge tube. Phenol $(400 \mu \mathrm{l})$ was added to each of the tubes and centrifuged for $20 \mathrm{~min}$ at $8,000 \mathrm{rpm}$. The supernatant were collected and transferred in new $1.7 \mu \mathrm{L}$ microcentrifuge tubes. Chloroform-Isoamyl $(24: 1)(400 \mu \mathrm{L})$ was added to each tube and the solutions were agitated manually until well mixed. The tubes were then centrifuged at 13,000 rpm for $5 \mathrm{~min}$. Supernatants were again collected and transferred in new $1.7 \mu \mathrm{L}$ microcentrifuge tubes, and $900 \mu \mathrm{L}$ of $100 \%$ ethanol and $40 \mu \mathrm{L}$ of $3 \mathrm{M}$ sodium acetate were added to each tube respectively. Solutions were agitated manually until well mixed and left overnight at $-20^{\circ} \mathrm{C}$. The following day, solutions were centrifuged for $10 \mathrm{~min}$ at 13,000 rpm. The ethanol solution was carefully taken out without disturbing the DNA pellet at the bottom of the tube. The DNA pellet was washed using $900 \mu \mathrm{L}$ of $70 \%$ ethanol prior to further centrifugation for $10 \mathrm{~min}$ at $13,000 \mathrm{rpm}$. Ethanol was carefully removed 
without disturbing the DNA pellet. The remaining ethanol was evaporated by placing the tubes containing the DNA pellets at $30^{\circ} \mathrm{C}$ for $30 \mathrm{~min}$. DNA pellets were re-suspended in $100 \mu \mathrm{L}$ Tris Ethylenediaminetetraacetic acid (TE - 10 mM Tris-HCl pH 8.0, 1 mM EDTA). Samples were immediately used for PCRs and stored at $4^{\circ} \mathrm{C}$ during the preparation process.

\section{PCR and library creation}

To specifically amplify bacterial DNA, $16 \mathrm{~S}$ universal primers Gray28F 5' GAGTTTGATCNTGGCTCAG 3' and Gray519r 5' GTNTTACNGCGGCKGCTG 3' (Ishak et al., 2011a, 2011b; McFrederick et al., 2012; Kautz et al., 2013b) were used. The primers amplify a $490 \mathrm{bp}$ sequence of the hyper variable V1-V3 region of the bacterial $16 \mathrm{~S}$ ribosomal RNA gene. To pool the samples together for 454 sequencing, we used Gray $28 \mathrm{~F}$ primers tagged on their 5' end with a unique 6-nucleotide barcodes generated by BARCLAW (Frank, 2009). Each barcode presented at least 3-nucleotide differences with each of the other barcodes to diminish the probability of later mis-assigning sequences to their sample of origin (Bystrykh, 2012) (Table 2.1).

Table 2.1: Tagged forward Gray $28 \mathrm{~F} 16 \mathrm{~S}$ universal bacteria primer sequences. Gray $28 \mathrm{~F}$ primers were given 6-nucleotid long tags on their 5' end. The 6-nucleotide long barcodes generated by BARCLAW are in bold characters.

\begin{tabular}{l|l} 
Primer name & \multicolumn{1}{|c}{ Primer sequence 5' $\rightarrow$ 3' } \\
\hline Gray28F-3 & TTCACAGAGTTTGATCNTGGCTCAG \\
Gray28F-17 & TGCTTAGAGTTTGATCNTGGCTCAG \\
Gray28F-20 & ATCCAAGAGTTTGATCNTGGCTCAG \\
Gray28F-24 & AACAGAGAGTTTGATCNTGGCTCAG \\
Gray28F-26 & ACAACAGAGTTTGATCNTGGCTCAG \\
Gray28F-30 & AGACTAGAGTTTGATCNTGGCTCAG \\
Gray28F-38 & CCGATAGAGTTTGATCNTGGCTCAG \\
Gray28F-45 & GGATAAGAGTTTGATCNTGGCTCAG
\end{tabular}

For each sample, PCR mix consisted of $15.0 \mu \mathrm{L}$ master mix solution containing approximately $20 \mathrm{ng}$ DNA suspension, $1 \mathrm{X}$ BIOTAQ Buffer, $0.4 \mathrm{mg} / \mathrm{mL}$ BSA, $0.2 \mathrm{mM}$

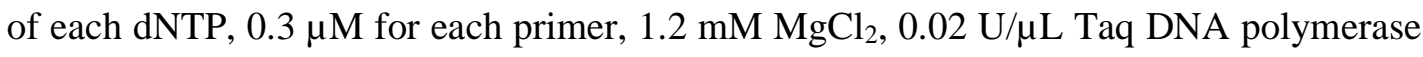
(Fisher Biotec) and $9.14 \mu \mathrm{L}$ molecular grade $\mathrm{ddH}_{2} \mathrm{O}$ (Sigma Aldrich). The PCR thermal cycling consisted of: an initial denaturation step at $94^{\circ} \mathrm{C}$ for $3 \mathrm{~min}$, followed by 30 cycles of denaturation at $94^{\circ} \mathrm{C}$ for $40 \mathrm{sec}$, annealing at $55^{\circ} \mathrm{C}$ for $40 \mathrm{~s}$ and extension at $72^{\circ} \mathrm{C}$ for $30 \mathrm{~s}$, and a final extension at $72^{\circ} \mathrm{C}$ for $5 \mathrm{~min}$. Amplifications of the $16 \mathrm{~S}$ gene were confirmed using agarose gel (1.5\%) electrophoresis and subsequent staining with ethidium 
bromide. Successful amplification of the target product was confirmed by comparison of the PCR bands with DNA ladder (Bioline HyperLadder ${ }^{\mathrm{TM}} 1 \mathrm{~kb}$ ). PCR products were cleaned using Agencourt AMPure XP beads (Beckman Coulter) following manufacturer's protocol. Agencourt AMPure XP beads were added to PCR product solutions (proportion added: $1.8 \mathrm{x}$ reaction volume) and mixed by pipetting. The solutions were left $5 \mathrm{~min}$ at room temperature. Then a magnet was attached to the side of each tube and left for $2 \mathrm{~min}$ until the solutions cleared. Clear solutions were retrieved and disregarded. Ethanol $70 \%$ $(200 \mu \mathrm{L})$ was added and tubes were left $30 \mathrm{sec}$ at room temperature. Ethanol was retrieved and disregarded. The magnet was removed and $45 \mu \mathrm{L}$ of molecular grade water added. Solutions were mixed by pipetting and magnets placed back on the side of each tube. Solutions containing cleaned PCR products were retrieved and placed into new $2 \mu \mathrm{L}$ PCR tubes. The PCR product quality and quantity were assessed using a modified high range curve of a Quant-iT TM PicoGreen ${ }^{\circledR}$ dsDNA Kit (Invitrogen) to insure the cleaned PCR product DNA quality and quantity met the 454 sequencing requirements. Three libraries for 454 sequencing were created. The libraries included six samples from Chapter 3. Each library consisted of a $100 \mu \mathrm{L}$ solution containing $500 \mathrm{ng}$ cleaned tagged PCR products from eight different samples preserved in TE Buffer. Each barcode was unique in a library to facilitate sample assignment of the sequences. The three libraries were sent to the Genomics Facility at University of Auckland (New Zealand) for 454 sequencing on GS Junior (Roche).

\subsubsection{Data Analysis}

Sequence analysis was realised using QIIME 1.7.0 (Caporaso et al., 2010b). Sequences were assigned to their sample of origin based on their barcodes using map files. Sequences were trimmed, and barcodes and primers removed with QIIME. Conserved sequences had a length comprised between $200 \mathrm{bp}$ and $1,000 \mathrm{bp}$, a minimum average quality score of 25 and a maximum homopolymer of 6 repeats. Sequences were clustered into Operational taxonomic units (OTUs) with 97\% identity similarity using Usearch v5.2.236 (Edgar, 2010). Sequences were denoised. Chimeras were disregarded after de novo chimera detection (Edgar, 2010). Singletons, i.e. OTUs containing only one sequence, were removed from analysis. The most abundant sequence in an OTU was selected as a representative of its cluster. Representative sequences were aligned against the Greengenes Core reference alignment (DeSantis et al., 2006) using PyNAST (Caporaso et al., 2010a). 
Representative sequence taxonomy was determined based on the Greengenes reference database v12.10 (McDonald et al., 2012) using RDP Classifier 2.2 (Wang et al., 2007). OTUs were identify as far as the genus level when possible. Bar charts and pies representing relative abundance of bacteria in each city / region and country were generated using $\mathrm{R} v$. i386 3.1.3. Samples were rarefied to 1,900 reads, the lowest number of reads found in a sample to allow comparison between countries, and to 2,000 reads to allow comparison between cities within New Zealand. Rarefaction curves and alpha diversity were generated with the QIIME pipeline, and beta-diversity calculated using UniFrac (Lozupone and Knight, 2005). Alpha diversity corresponds to the diversity of OTUs within samples, and beta diversity to the diversity of OTUs between samples. Australia was removed from later analysis as only one sample was available for that country. Differences between samples was tested based on the distances between samples by the non-parametric method Adonis in QIIME 1.7.0. G-tests were realized in QIIME 1.7.0 to analyse the difference in relative abundance of bacteria between countries. Venn diagrams to compare bacterial diversity between samples were generated using the package VennDiagram in R v. i386 3.1.3. A test was consider significant when $\mathrm{p}<0.05$.

\subsection{Results}

\subsubsection{4 read quality}

To characterise and compare bacterial diversity present in Argentine ants between the ant home range and three invaded countries, 16S bacterial DNA amplified from Argentine ant samples from four different countries were sequenced using 454 sequencing technology. A total of 273,798 sequence reads was obtained from the three resulting libraries. The libraries contained respectively 138,672, 62,897 and 72,229 reads and included reads from 6 extra samples from a different analyse (see Chapter 3). A total of 565 reads were excluded based on their length, either below 200 or above 1,000 nucleotides. A further 261 reads were excluded for their number of ambiguous bases exceeding 6 nucleotides, 10,118 reads for a mean quality score below 25, and 5,316 reads for presenting a homopolymer run exceeding 6. After these quality controls, a total of 89,076 reads were assigned to the 18 samples. Ten samples corresponded to ten unique cities / area from Argentina, the United States and Australia, and 8 samples corresponded to four New Zealand cities sampled each twice (once in 2011 and once in 2012). Between 2,021 to 9,313 reads (mean $=4,949 \pm 518$ ) were 
assigned. A final denoising, chimera and singleton exclusion step with Usearch 7.0.1090 left a total of 85,915 reads. These reads were assigned to the 18 samples, from 1,944 to 9,017 reads per sample (mean $=4,773 \pm 509$ ), and were clustered into 763 Operational taxonomic units (OTUs) with $97 \%$ identity similarity between reads.

\subsubsection{Bacterial diversity among and between countries}

Alpha diversity was analysed through rarefaction curves based on the number of observed number of species for each country, considering each sampled city / area as a pseudorepetition (Figure 2.2).

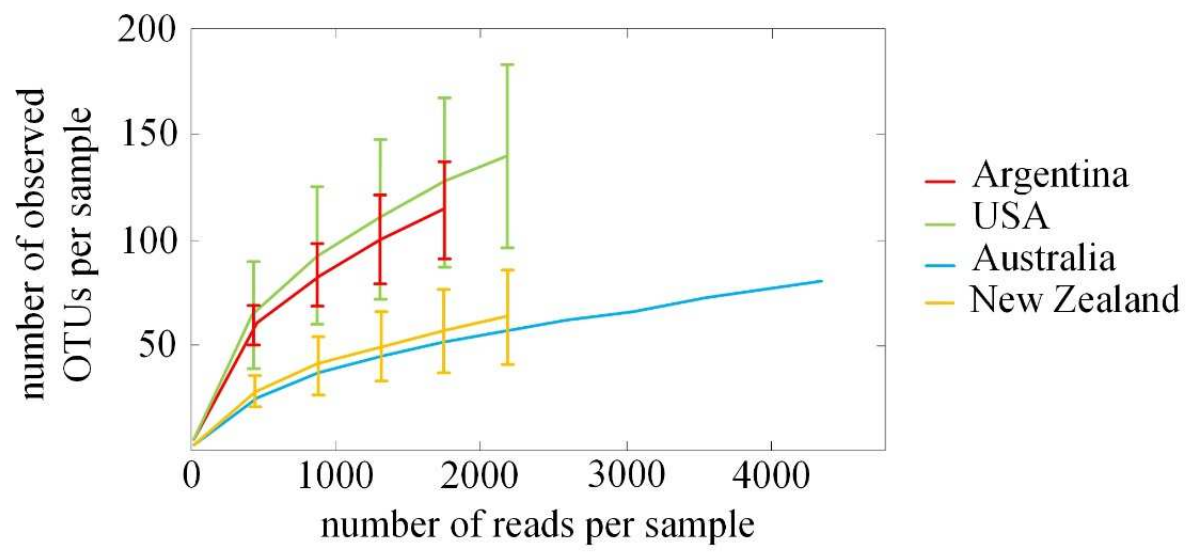

Figure 2.2: Rarefaction curves using QIIME 1.7.0, based on the estimated number of OTUs in Argentine ants in the ant home range and three invaded countries based on the number of OTUs present in each country. For each city in a country, each sample was considered a pseudo-replicate sample of that country. No error bars for the standard variation is presented for Australia as only one city was sampled. The length of the rarefaction curve for each country is limited by the lowest number of reads in a sample from that country.

The observed number of OTUs is higher in the ant home range Argentina and primary invaded country, the United States, and lower in secondary and tertiary invaded countries, Australia and New Zealand, respectively. Furthermore the number of observed OTUs increased with the number of reads for each country. The plateaux of the curves were not reached, indicating that more than the number of reads per sample obtained in this study is needed to expose the full OTUs diversity present in each country. However, the slopes of the curves are slowing and are closed to reaching the plateaux (Figure 2.2). This suggests that the sampling is sufficient to identify most of the OTUs present in the ants in each country, even at the rarefaction of 1,900 reads later used for comparative analysis. Multiple 
t-tests indicated a significant difference in both the average Chaol richness and average number of species observed between Argentina and New Zealand (Bonferroni corrected $\mathrm{p}<0.05$ and $\mathrm{p}<0.05$ respectively), and between the United States and New Zealand (Bonferroni corrected $\mathrm{p}<0.05$ for both country). The average Chao1 richness did not differ between Argentina and Australia (Bonferroni corrected $\mathrm{p}=0.108$ ).

To compare bacterial diversity between countries, beta diversity was analysed using principal coordinate analyses (PCoA) of unweighted and weighted UniFrac distances between samples after rarefaction of each sample to 1,900 reads, under the lowest number of reads in a sample (Figure 2.3).
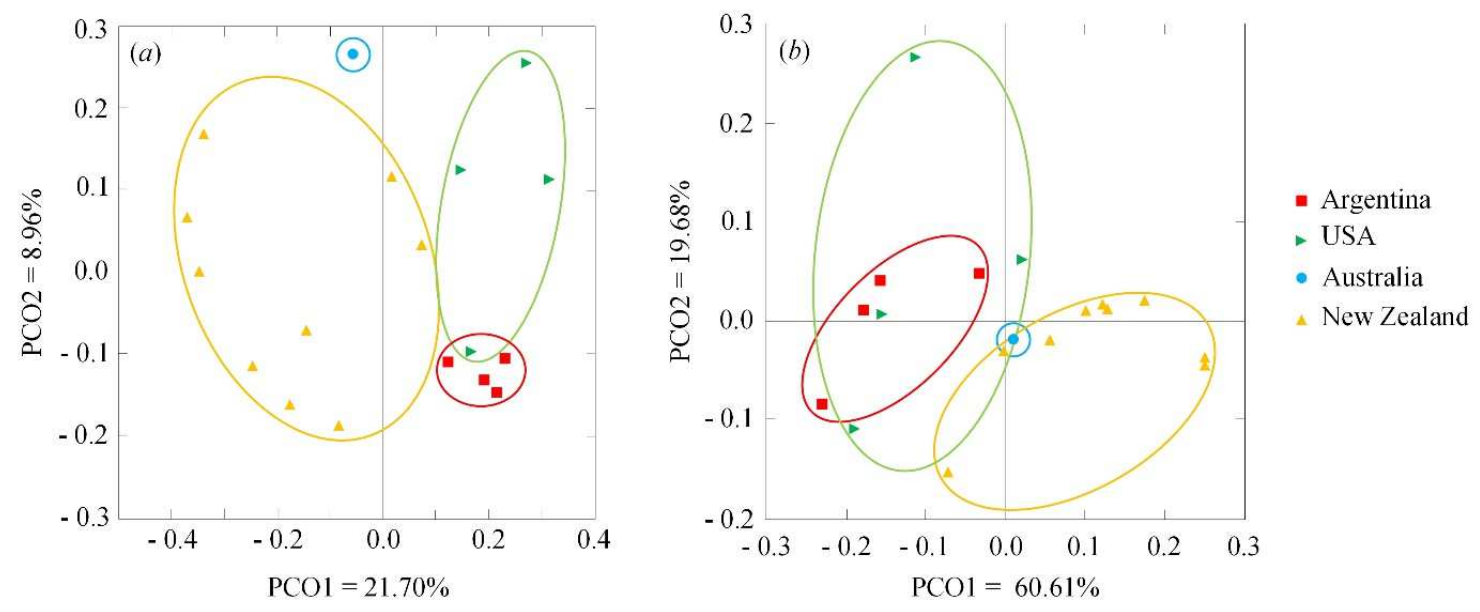

Figure 2.3: Principal coordinate analysis (PCoA) based on a) unweighted and b) weighted phylogenetic UniFrac distances generated in QIIME 1.7.0 between Argentine ant samples from four countries. Reads were rarefied to 1,900 to allow comparison between samples. Circles were manually added to visualise the dispersion of samples from a same country.

Country of origin is a significant factor influencing the distance between samples (Adonis, unweighted, $\mathrm{R}^{2}=0.29, \mathrm{p}<0.05$; and weighted, $\left.\mathrm{R}^{2}=0.47, \mathrm{p}<0.05\right)$.

Unclassified OTUs, meaning OTUs outside the bacterial domain, and OTUs with undetermined bacteria phylum were marginal ( $<0.05 \%$ of each sample reads). In total, 181 bacteria genera belonging to 16 different phyla were detected across all countries. Six phyla and 19 genera were shared by all countries (Figure 2.4). 

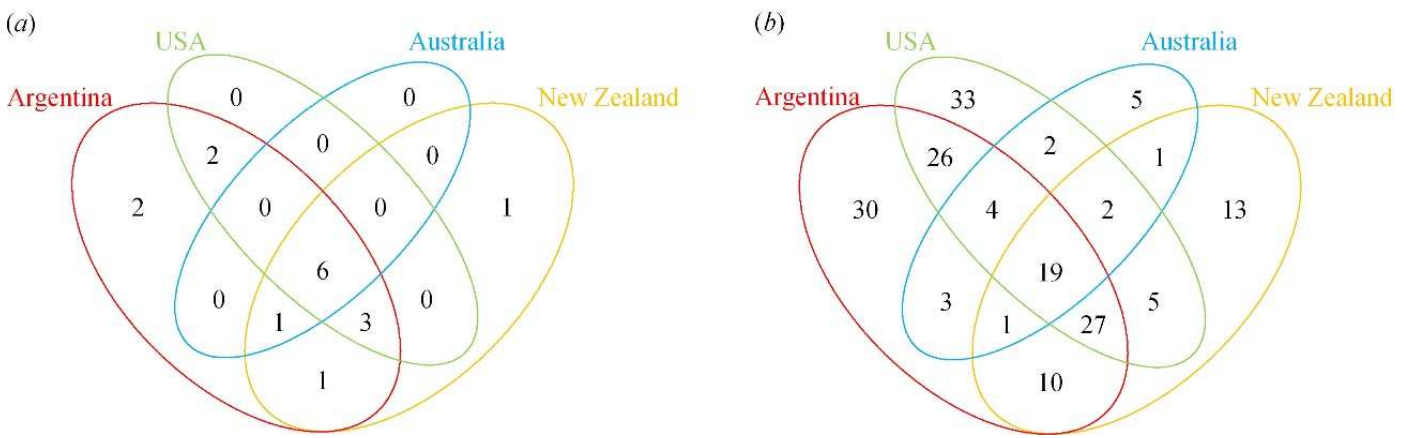

Figure 2.4: Venn diagrams of unique and shared bacteria present in Argentine ants in the ant home range and three invaded countries: a) at the phylum level, and b) at the genus level. Only the presence and absence of bacteria defined to the phylum level in a) or to the genera level in b) were considered. Venn diagrams were generated with the package Venn diagram in $\mathrm{R}$ (what version).

Argentina and the United States present a higher number of unique phyla and genera than Australia and New Zealand (Figure 2.4).

Looking more specifically at the identity and the relative abundance of the 16 phyla, only two phyla were present $>1 \%$ in relative abundance, and therefore considered non-rare phyla, in all four countries: Proteobacteria and Firmicutes (Table 2.2). 
Table 2.2: Bacterial phyla in the Argentine ant home range, Argentina, and three invaded countries, the United States, Australia and New Zealand. The mean of the relative abundance of each phylum is presented in percentage and the mean of the raw number of reads identified for each phylum is in brackets. For Australia, no means were calculated as only one sample represented the country; the relative abundance and the number of reads corresponded to the data from that single sample. Phyla present $>1 \%$ in at least one treatment are considered non rare and are in bold. Phyla present in all countries are indicated by $\wedge$. OTUs that could not be classified as bacteria (category "Unclassified") and OTUs with non-named phyla are marginal, representing $<0.05 \%$ in total in each countries. In order to compare bacteria presence between countries, g-tests were performed on the relative abundance of the different phyla after rarefaction to 1,900 reads. Bonferroni corrected p-values are presented for each phylum. Significant pvalues are indicated by a *. Australia was not included in the g-tests as the country was only represented by one sample.

\begin{tabular}{|c|c|c|c|c|c|}
\hline \multirow{2}{*}{ Phylum } & \multicolumn{4}{|c|}{ Country } & \multirow{2}{*}{ Bonferroni corrected p-value } \\
\hline & Argentina & USA & Australia & New Zealand & \\
\hline Unclassified & $0.00(0.00)$ & $0.02(1.00)$ & $0.00(0.00)$ & $0.01(0.22)$ & NA \\
\hline Other phylum & $0.06(3.50)$ & $0.03(1.25)$ & $0.03(2.00)$ & $0.01(0.56)$ & 1 \\
\hline Acidobacteria^$^{\wedge}$ & $0.25(13.75)$ & $0.16(7.25)$ & $0.20(15.00)$ & $0.01(0.33)$ & 1 \\
\hline Actinobacteria $^{\wedge}$ & $0.47(25.75)$ & $2.22(101.5)$ & $0.54(40.00)$ & $0.18(7.60)$ & $5.24 \mathrm{e}^{-08 *}$ \\
\hline Armatimonadetes & $0.05(3.00)$ & $0.03(1.50)$ & $0.00(0.00)$ & $0.00(0.11)$ & NA \\
\hline Bacteroidetes^^ & $0.21(11.50)$ & $0.63(28.75)$ & $0.11(8.00)$ & $0.07(3.11)$ & $0.03 *$ \\
\hline Chloroflexi & $0.81(44.50)$ & $0.02(0.75)$ & $0.00(0.00)$ & $0.00(0.00)$ & NA \\
\hline Cyanobacteria $^{\wedge}$ & $0.34(18.50)$ & $0.09(4.25)$ & $0.03(2.00)$ & $0.10(4.22)$ & 1 \\
\hline Firmicutes $^{\wedge}$ & $\mathbf{3 5 . 8 8}(1971.25)$ & $\mathbf{4 0 . 4 8}(1847.50)$ & $\mathbf{3 0 . 8 7}(2268.00)$ & $18.78(800.00)$ & $8.44 \mathrm{e}^{-48 *}$ \\
\hline Fusobacteria & $0.02(1.00)$ & $0.01(0.25)$ & $0.00(0.00)$ & $0.00(0.11)$ & NA \\
\hline Gemmatimonadetes & $0.01(0.50)$ & $0.00(0.00)$ & $0.00(0.00)$ & $0.00(0.00)$ & NA \\
\hline Planctomycetes & $0.00(0.25)$ & $0.01(0.25)$ & $0.00(0.00)$ & $0.00(0.00)$ & NA \\
\hline Proteobacteria $^{\wedge}$ & $\mathbf{5 9 . 5 8}(3273.25)$ & $\mathbf{5 6 . 2 9}(2569.00)$ & $\mathbf{6 8 . 2 0}(5010.00)$ & $80.81(3442.33)$ & $5.95 \mathrm{e}^{-28 *}$ \\
\hline TM7 & $0.03(1.75)$ & $0.00(0.00)$ & $0.00(0.00)$ & $0.00(0.00)$ & NA \\
\hline Tenericutes & $\mathbf{2 . 2 3}(122.75)$ & $0.00(0.00)$ & $0.01(1)$ & $0.01(0.33)$ & NA \\
\hline Thermi & $0.03(1.50)$ & $0.00(0.00)$ & $0.00(0.00)$ & $0.01(0.33)$ & NA \\
\hline WPS-2 & $0.00(0.00)$ & $0.00(0.00)$ & $0.00(0.00)$ & $0.01(0.22)$ & NA \\
\hline WYO & $0.02(1)$ & $0.02(1)$ & $0.00(0.00)$ & $0.00(0.11)$ & NA \\
\hline
\end{tabular}

These two phyla were also the two most abundant phyla in every countries, representing in average $31.50 \%$ for Firmicutes, and $61.22 \%$ for Proteobacteria of the bacterial phyla. Actinobacteria and Tenericutes are the two other sole phyla present $>1 \%$ in at least one country, respectively in the United States (2.22\%) and in Argentina (2.23\%). Acidobacteria $($ mean $=0.16 \%)$, Bacteroidetes $($ mean $=0.26 \%)$ and Cyanobacteria $($ mean $=0.14 \%)$ are the only rare phyla present in all countries. Only Argentina and New Zealand presented unique phyla. Argentine ants in their home range Argentina were the only ones infected with the rare phyla TM7 $(0.03 \%)$ and Gemmatimonadetes $(0.01 \%)$, while New Zealand ants were the only ones infected by the rare WPS-2 $(0.01 \%)$. G-tests were used to examine the difference in relative abundance of each bacteria phylum between Argentina, the United 
States and New Zealand. Australia was not included as only one sample represented the country. Samples were rarefied to 1,900 reads (the lowest number of reads in a sample) each to allow comparison between countries. Bonferroni corrected p-values indicate significant differences between Argentina, the United States and New Zealand (Table 2.2). These differences between the countries were related to three phyla: Actinobacteria, Firmicutes and Proteobacteria (Bonferroni corrected $\mathrm{p}<0.05$ for the three phyla).

Of the 181 identified genera, 19 bacterial genera were shared between all four countries (Figure 2.5). However, 2 of these 19 bacterial genera were not named with conventional names (not including genera with names not conventionally defined yet), and were therefore not considered in the summary table of bacterial genera present in all countries (Table 2.3). 
Table 2.3: Bacterial genera in Argentine ant home range and three invaded countries. Only bacteria with a conventional names are presented. The relative abundance mean of genera is presented in percentage and the mean of raw number of reads identified for each genus is in brackets. For Australia, no means were calculated as only one sample represented the country, the relative abundance and the number of reads corresponded to the data from that single sample. Genera present $>1 \%$ at least in one country are indicated by $\wedge$, and genera present $>1 \%$ in all countries are in bold. Relative abundances $>1 \%$ are in bold. In order to compare bacteria presence between countries, g-tests was undertaken on the relative abundance and on the number of reads of different genera after rarefaction to 1,900 reads. Bonferroni corrected p-values are presented for each genus. Significant p-values are indicated by a *. Australia was not included in the g-tests as the country was only represented by one sample.

\begin{tabular}{|c|c|c|c|c|c|c|}
\hline \multirow{2}{*}{ phylum } & \multirow{2}{*}{ genus } & \multicolumn{4}{|c|}{ Country } & \multirow{2}{*}{$\begin{array}{c}\text { Bonferroni } \\
\text { corrected } \\
\text { p-value }\end{array}$} \\
\hline & & Argentina & USA & Australia & New Zealand & \\
\hline Actinobacteria & Tsukamurella & $0.03(1.50)$ & $0.03(1.50)$ & $0.01(1)$ & $0.07(2.89)$ & NA \\
\hline \multirow{3}{*}{ Bacteroidetes } & Chryseobacterium & $0.08(4.50)$ & $0.08(3.50)$ & $0.03(2.00)$ & $0.01(0.44)$ & 1 \\
\hline & Flavisolibacter & $0.01(0.75)$ & $0.03(1.25)$ & $0.01(1)$ & $0.01(0.56)$ & NA \\
\hline & Segetibacter & $0.02(1.25)$ & $0.16(7.50)$ & $0.04(3.00)$ & $0.01(0.44)$ & NA \\
\hline Firmicutes & Lactobacillus $^{\wedge}$ & $33.79(1856.50)$ & $38.45(1754.75)$ & $\mathbf{3 0 . 5 7}(2246.00)$ & 18.65 (794.44) & $7.31 \mathrm{e}^{-42 *}$ \\
\hline \multirow{12}{*}{ Proteobacteria } & Caulobacter & $0.19(10.25)$ & $0.21(9.50)$ & $0.01(1.00)$ & $0.04(1.89)$ & 1 \\
\hline & Phenylobacterium & $0.00(0.25)$ & $0.01(0.25)$ & $0.01(1)$ & $0.00(0.11)$ & NA \\
\hline & Bradyrhizobium $^{\wedge}$ & $\mathbf{3 . 1 6}(173.75)$ & $\mathbf{2 . 3 2}(106)$ & $0.31(23)$ & $0.30(12.89)$ & $2.96 \mathrm{e}^{-08 *}$ \\
\hline & Methylobacterium & $0.15(8.00)$ & $0.25(11.50)$ & $0.01(1)$ & $0.03(1.44)$ & 1 \\
\hline & Gluconobacter $^{\wedge}$ & $22.04(1211.00)$ & $21.75(992.75)$ & $45.89(3371.00)$ & $\mathbf{6 0 . 7 2}(2586.56)$ & $3.11 \mathrm{e}^{-151 *}$ \\
\hline & Achromobacter & $0.26(14.25)$ & $0.35(15.75)$ & $0.04(3.00)$ & $0.12(5.00)$ & 1 \\
\hline & Delftia $^{\wedge}$ & $\mathbf{1 . 8 3}(100.75)$ & $1.70(77.75)$ & $0.11(8.00)$ & $0.34(14.33)$ & $3.18 \mathrm{e}^{-05 *}$ \\
\hline & Ralstonia & $0.05(2.50)$ & $0.14(6.25)$ & $0.01(1.00)$ & $0.02(0.78)$ & 1 \\
\hline & Escherichia & $0.13(7.25)$ & $0.19(8.50)$ & $0.01(1.00)$ & $0.02(0.78)$ & NA \\
\hline & Acinetobacter ${ }^{\wedge}$ & $\mathbf{3 . 6 3}(199.25)$ & $4.21(192.00)$ & $0.65(48.00)$ & $0.27(11.56)$ & $9.85 \mathrm{e}^{-28 *}$ \\
\hline & Pseudomonas $^{\wedge}$ & $2.04(112.25)$ & $\mathbf{2 . 0 2}(92.00)$ & $0.05(4.00)$ & $0.29(12.56)$ & $3.45 \mathrm{e}^{-08 *}$ \\
\hline & Stenotrophomonas ${ }^{\wedge}$ & $0.87(48.00)$ & $\mathbf{1 . 0 9}(49.75)$ & $0.10(7.00)$ & $0.19(8.11)$ & $10.64 \mathrm{e}^{-4 *}$ \\
\hline \multicolumn{2}{|c|}{$\begin{array}{l}\text { Total of relative abundance of OTUs } \\
\text { (number of reads) conventionally } \\
\text { named genera present in all countries }\end{array}$} & $68.30(3751.75)$ & $72.97(3330.75)$ & $77.89(5721.00)$ & $81.10(3454.33)$ & \\
\hline \multicolumn{2}{|c|}{$\begin{array}{l}\text { Total of relative abundance of OTUs } \\
\text { (number of reads) conventionally } \\
\text { named and not yet conventionally } \\
\text { named genera (including the } \\
\text { conventionally named genera present } \\
\text { in all countries) }\end{array}$} & $83.45(4584.50)$ & $81.65(3726.50)$ & $78.80(5789)$ & $81.98(3492)$ & \\
\hline \multicolumn{2}{|c|}{$\begin{array}{l}\text { Total of relative abundance of OTUs } \\
\text { (number of reads) not defined to the } \\
\text { genera level }\end{array}$} & $16.55(909.25)$ & $18.35(837.75)$ & $21.20(1557)$ & $18.02(767.67)$ & \\
\hline
\end{tabular}

The lack of replication for samples from Australia may have led to an underestimation of the number of core bacteria genera present in Argentine ants. On average, $18.53 \%$ of OTUs were not defined to the bacteria genus. The most prevalent genera present in all countries were Gluconobacteria and Lactobacillus. Together the two bacterial genera represented in every country $>50 \%$ in relative abundance of the bacteria reads. Only four other bacterial genera were present $>1 \%$ in at least in one country: in Argentina and the United States, Bradyrhizobium (3.16\% and $2.32 \%$ respectively), Acinetobacter (3.63\% and $4.21 \%)$, 
Delftia (1.83\% and $1.70 \%)$ and Pseudomonas (2.04 and 2.02\%), and only in the United States, Stenotrophomonas (1.09\%). G-tests were undertaken between Argentina, the United States and New Zealand to test the difference in relative abundance each bacteria genus. Australia was not included as only one sample represented the country. Samples were rarefied to 1,900 reads (the lowest number of read in one sample) each to allow comparison between countries. Beferroni corrected p-value indicated significant differences between the three countries for the relative abundance of seven genera: Lactobacillus, Bradyrhizobium, Gluconobacter, Delftia, Acinetobacter, Pseudomonas, and Stenotrophomonas (Table 2.3). Lactobacillus relative abundance was higher in Argentina and the United States (respectively 33.79\% and 38.45\%) than in Australia and New Zealand (respectively 30.57 and $18.65 \%$ ). At the contrary, Gluconobacter relative abundance was lower in Argentina and the United States (respectively 22.04\% and 21.75\%) than in Australia and New Zealand (respectively $45.89 \%$ and $60.72 \%$ ).

The number of bacteria genera was higher in Argentina and the United States than in Australia and New Zealand when looking at the number of bacteria named to the genus level and present $>1 \%$ (Figure 2.5). 


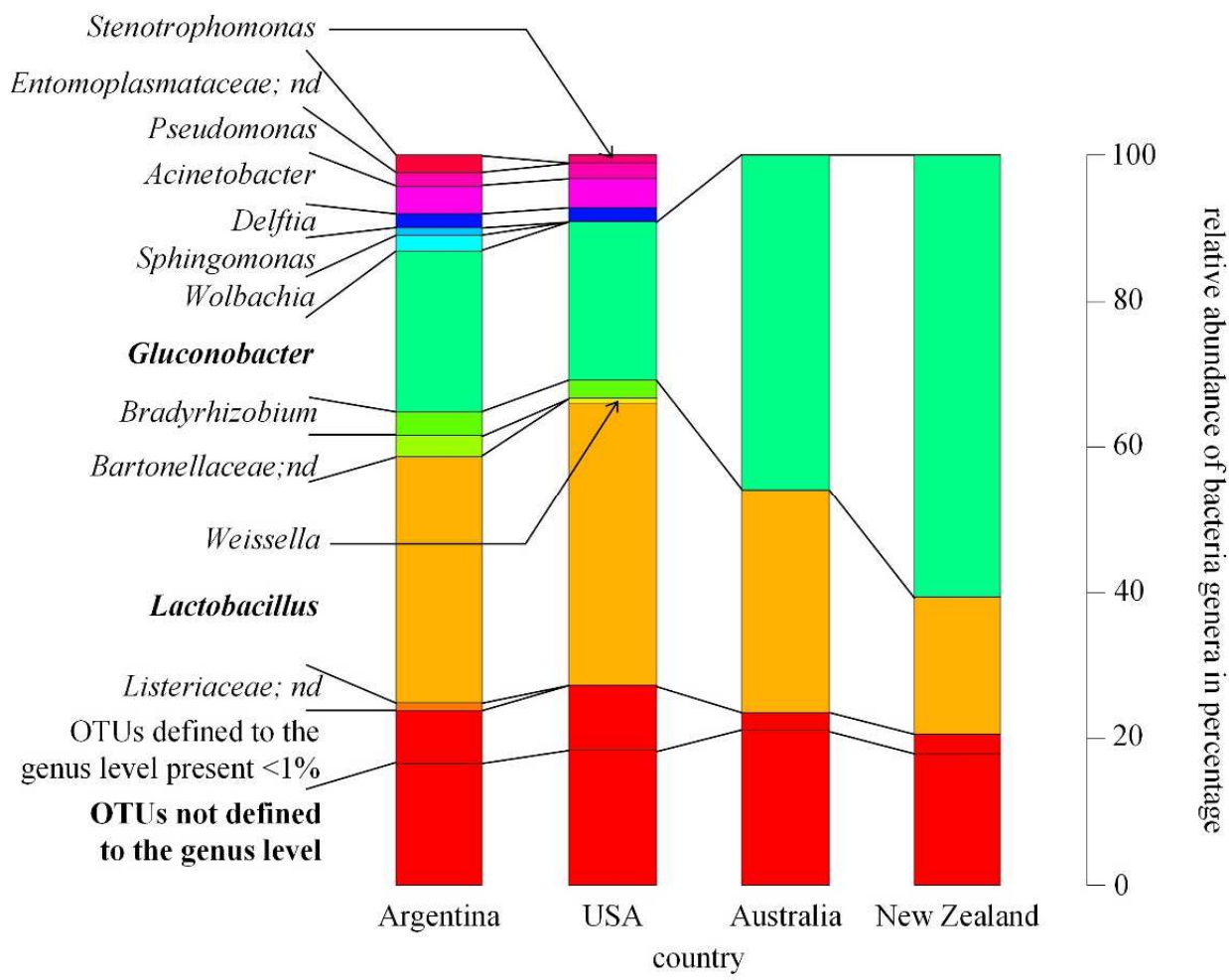

Figure 2.5: Relative abundance of non-rare bacterial genera in Argentine ants in the ant home range and three invaded countries. Only non- rare bacteria, bacteria present $>1 \%$ in a country, are presented. Bacteria noted "nd" are identify to the genus level however, these genera are not conventionally named yet. Genera present $>10 \%$ in at least one country are in bold. The countries are ordered from left to right: from the home range Argentina, to invaded countries which populations occurred after a primary invasion, the United States (USA), after a secondary invasion, Australia, and a tertiary invasion, New Zealand.

Argentina presented 11 non-rare bacteria, and the United States eight. Only Lactobacillus and Gluconobacter were non-rare bacteria present in Australia and New Zealand.

Furthermore the diversity present in each country can be observed by the variation in the number of non-rare genera present in each sampled city / area (Figure 2.6). Core bacteria were defined as bacteria present in all sites and all cities. Only nine genera were present in all sites (Table 2.4). They represented $>50 \%(\min 54.23 \%-\max 92.06 \%)$ of the bacteria present in the ants in each city. Core bacteria in Argentine ants present under the previous description are therefore: Lactobacillus, Caulobacter, Bradyrhizobium, Gluconobacter, Achromobacter, Delftia, Acinetobacter, Pseudomonas, and Stenotrophomonas. Lactobacillus and Gluconobacter corresponded to the main bacterial genera in each city. 


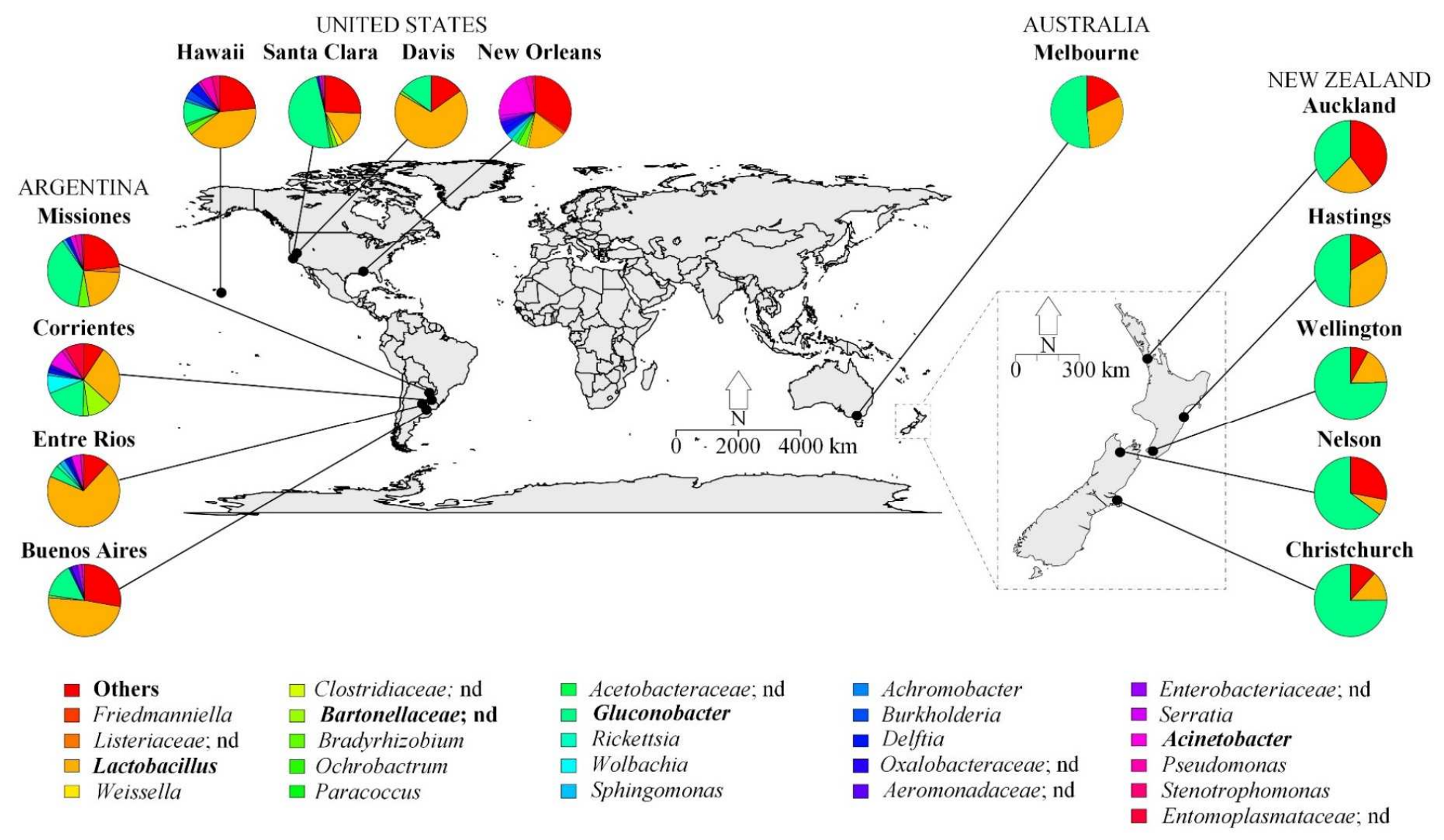

Figure 2.6: Bacterial diversity at the genus level present in each Argentine ant sampling site in the Argentine ant home range and three invaded countries. Only non-rare bacteria (present at $>1 \%)$ identified to the genus level in a site are presented. All rare $(<1 \%)$ and non-identified bacteria were grouped in the category "Others". Bacteria present $>10 \%$ in at least one site are in bold. Bacteria names noted "nd" indicate that the genus has not yet been conventionally named. Bacterial diversity is higher in Argentina, the ant home range, and the United States, a primary invaded country, than in Australia and New Zealand, respectively secondary and tertiary invaded countries. 
Table 2.4: Relative abundance in percentage of bacterial genera present in Argentine ants in all cities. Only bacterial genera present in all cities are considered part of the core bacteria of Argentine ants and are presented in the table. Genera present $>1 \%$ at least in one city are indicated by $\wedge$, and genera present $>1 \%$ in all countries are in bold. Relative abundances $>1 \%$ are in bold. As no replicates were performed for the each sites but for four cities in New Zealand, no statistical test could be undertaken to compare the difference of relative abundance of the core bacteria between cities.

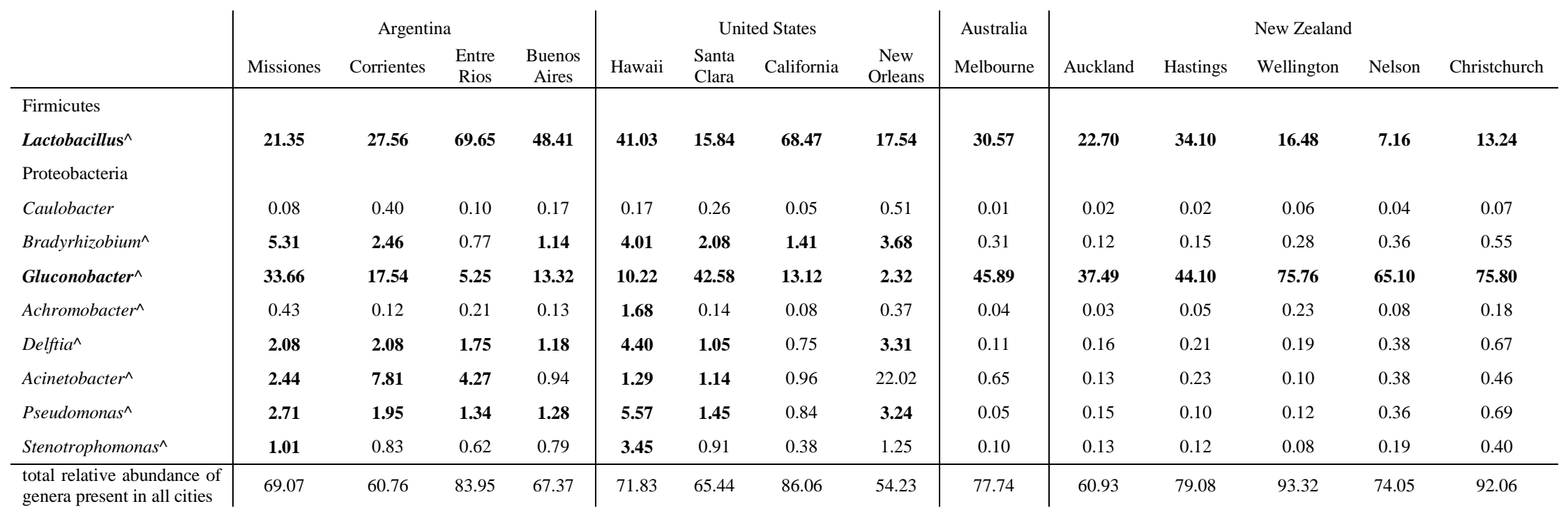




\subsubsection{Bacterial diversity within New Zealand}

Alpha diversity was analysed through rarefaction curves based on the number of observed number of species for five cities in New Zealand (Figure 2.7).

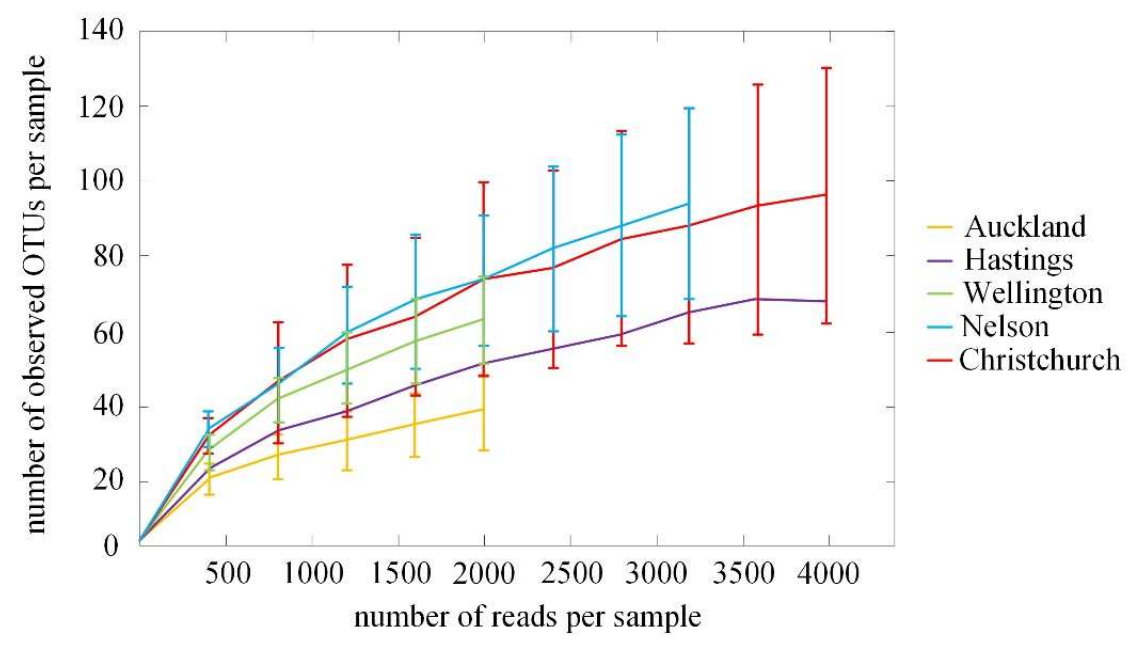

Figure 2.7: Rarefaction curves using QIIME 1.7.0, based on the estimated number of observed OTUs in Argentine ants in New Zealand between 2011 and 2012. Cities in the legend are present in order from North to South. For each city, each year sampling represented a replicate sample of that city. No error bars for the standard variation is presented for Hastings as the city was sampled only once. The length of the rarefaction curve for each city is limited by the lowest number of sequence reads in a sample from that city.

Replicates in a city corresponded to two consecutive sampling, in 2011 and 2012, except for Hasting where only one sample was available. The number of observed bacteria increased with the number of reads for each city. The plateaux of the curves were not reached indicating that more than the number of reads per sample obtained in this study are needed to expose the full OTU diversity present in each city. However, the slopes of the curves are slowing and are closed to reaching the plateaux (Figure 2.8). This result suggests that the sampling is sufficient to identify most of the bacteria present in the ants in each city, even at the rarefaction of 2,000 reads later used for comparative analysis. The observed number of OTUs seems higher in the Southern cities, Nelson and Christchurch, then in the Northern cities, Auckland, Hastings and Wellington. However, the variation in the number of observed OTUs in each city was high and did not allow to visually conclude any significant differences between the cities. Multiple t-tests indicated no significant differences for the number of observed OTUs between cities (Adonis, Bonferroni corrected 
p $>0.05$ for all pairs not including Hastings). Similarly, no significant differences were observed for Chao1 richness indicator between cities (Adonis, Bonferroni corrected p $>0.05$ for all pairs not including Hastings.

To compare bacterial diversity between cities in New Zealand, beta diversity was analysed through principal coordinate analyses (PCoA) of unweighted and weighted UniFrac distances between samples after rarefaction of each sample to 2,000 reads, under the lowest number of reads in a sample (Figure 2.8).
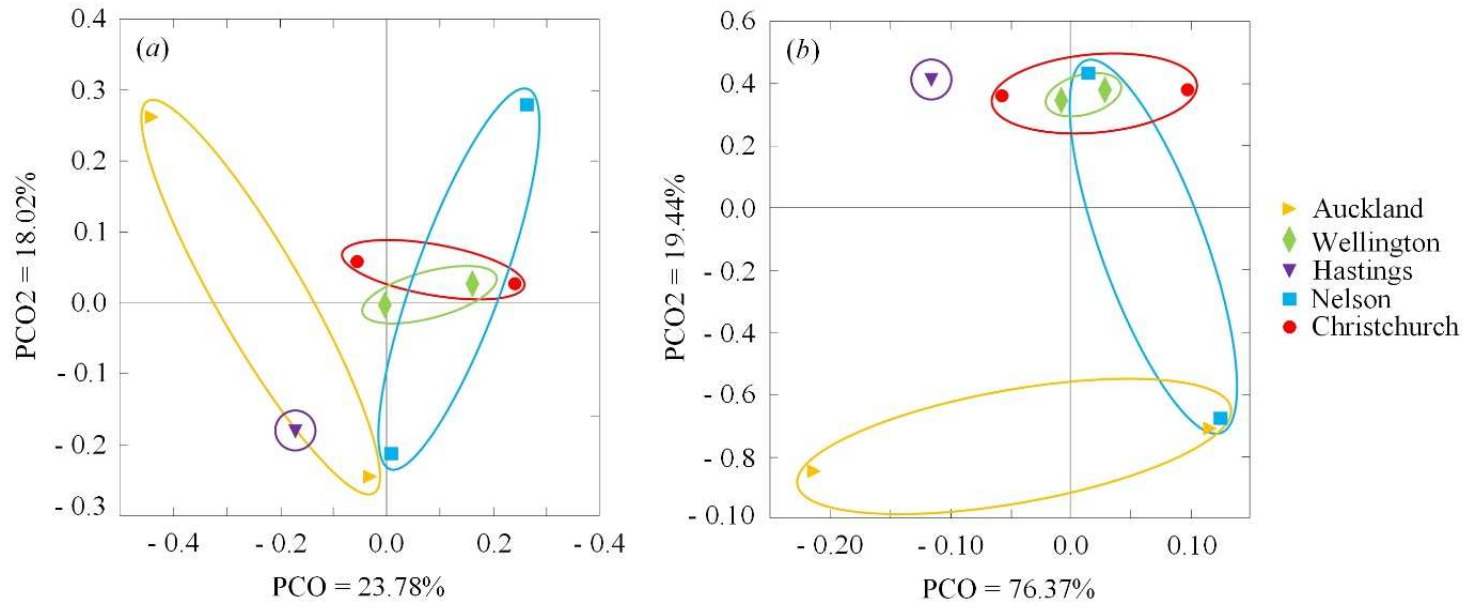

Figure 2.8: Principal coordinate analysis (PCoA) based on a) unweighted and b) weighted phylogenetic UniFrac distances generated in QIIME 1.7.0 between Argentine ants from five cities in New Zealand. Cities in the legend are present in order from North to South. Reads were rarefied to 2,000 (lowest number of reads in a sample) to allow comparison between samples. Circles were manually added to visualise the dispersion of samples from a same city. Only one sample was available for Hastings.

City of origin was not a significant factor influencing the distance between samples (Adonis, unweighted, $\mathrm{R}^{2}=0.37, \mathrm{p}=0.94$; and weighted, $\mathrm{R}^{2}=0.31, \mathrm{p}=0.80$ ).

In New Zealand, 12 bacteria phyla were identified in Argentine ant samples from the five sampling cities (Table 2.5). 
Table 2.5: Bacterial phyla present in Argentine ants in New Zealand. The mean of the relative abundance of each phylum is presented in percentage and the mean of raw number of reads for each phylum is in brackets. For Hastings, no means were calculated as only one sample represented the city; the relative abundance and the number of reads corresponded to the data from that single sample. Phyla present $>1 \%$ at least in one city are indicated by ${ }^{\wedge}$, and phyla present $>1 \%$ in all cities are in bold. Relative abundances $>1 \%$ are in bold. In order to compare bacteria presence between cities, g-tests were realized on the relative abundance and on the number of reads of different genera after rarefaction to 2,000 reads. Bonferroni corrected p-values are presented for each phylum first for the relative abundance and in brackets for the number of reads. Significant p-values are indicated by a *. Hastings was not included in the g-tests as the city was only represented by one sample.

\begin{tabular}{|c|c|c|c|c|c|c|}
\hline \multirow{2}{*}{ Phylum } & \multicolumn{5}{|c|}{ City } & \multirow{2}{*}{$\begin{array}{l}\text { Bonferroni } \\
\text { corrected } P \text { - } \\
\quad \text { value }\end{array}$} \\
\hline & Auckland & Hastings & Wellington & Nelson & Christchurch & \\
\hline Unclassified & $0.00(0)$ & $0.00(0)$ & $0.01(1)$ & $0.00(0)$ & $0.01(1)$ & NA \\
\hline Other phyla & $0.02(1)$ & $0.01(1)$ & $0.01(1)$ & $0.00(0)$ & $0.02(2)$ & NA \\
\hline Acidobacteria & $0.00(0)$ & $0.00(0)$ & $0.00(0)$ & $0.04(3)$ & $0.00(0)$ & NA \\
\hline Actinobacteria^^ & $0.07(4)$ & $0.02(2)$ & $0.05(4)$ & $0.46(36)$ & $0.27(23)$ & 0.32 \\
\hline Armatimonadetes & $0.00(0)$ & $0.00(0)$ & $0.00(0)$ & $0.01(1)$ & $0.00(0)$ & NA \\
\hline Bacteroidetes $^{\wedge}$ & $0.03(2)$ & $0.04(3)$ & $0.01(1)$ & $0.18(14)$ & $0.09(8)$ & 1 \\
\hline Cyanobacteria & $0.00(0)$ & $0.04(3)$ & $0.14(11)$ & $0.21(16)$ & $0.09(8)$ & NA \\
\hline Firmicutes $^{\wedge}$ & $22.80(1382)$ & 34.21 (2829) & $\mathbf{1 6 . 5 7}(1279)$ & $7.35(573)$ & 13.39 (1137) & $7.54 \mathrm{e}^{-61 *}$ \\
\hline Fusobacteria & $0.02(1)$ & $0.00(0)$ & $0.00(0)$ & $0.00(0)$ & $0.00(0)$ & NA \\
\hline Proteobacteria $^{\wedge}$ & 77.07 (4671) & $65.67(5430)$ & 83.13 (6418) & 91.71 (7151) & $86.11(7311)$ & $3.90^{\mathrm{e}-11 *}$ \\
\hline Tenericutes & $0.00(0)$ & $0.01(1)$ & $0.03(2)$ & $0.00(0)$ & $0.00(0)$ & NA \\
\hline Thermi & $0.00(0)$ & $0.00(0)$ & $0.04(3)$ & $0.00(0)$ & $0.00(0)$ & NA \\
\hline WPS-2 & $0.00(0)$ & $0.00(0)$ & $0.00(0)$ & $0.03(2)$ & $0.00(0)$ & NA \\
\hline WYO & $0.00(0)$ & $0.00(0)$ & $0.00(0)$ & $0.01(1)$ & $0.00(0)$ & NA \\
\hline
\end{tabular}

The higher number of phyla was in Nelson (nine phyla) and the lower in Auckland and Christchurch (five phyla). Four phyla were shared between the five cities (Table 2.5 and Figure 2.9). 

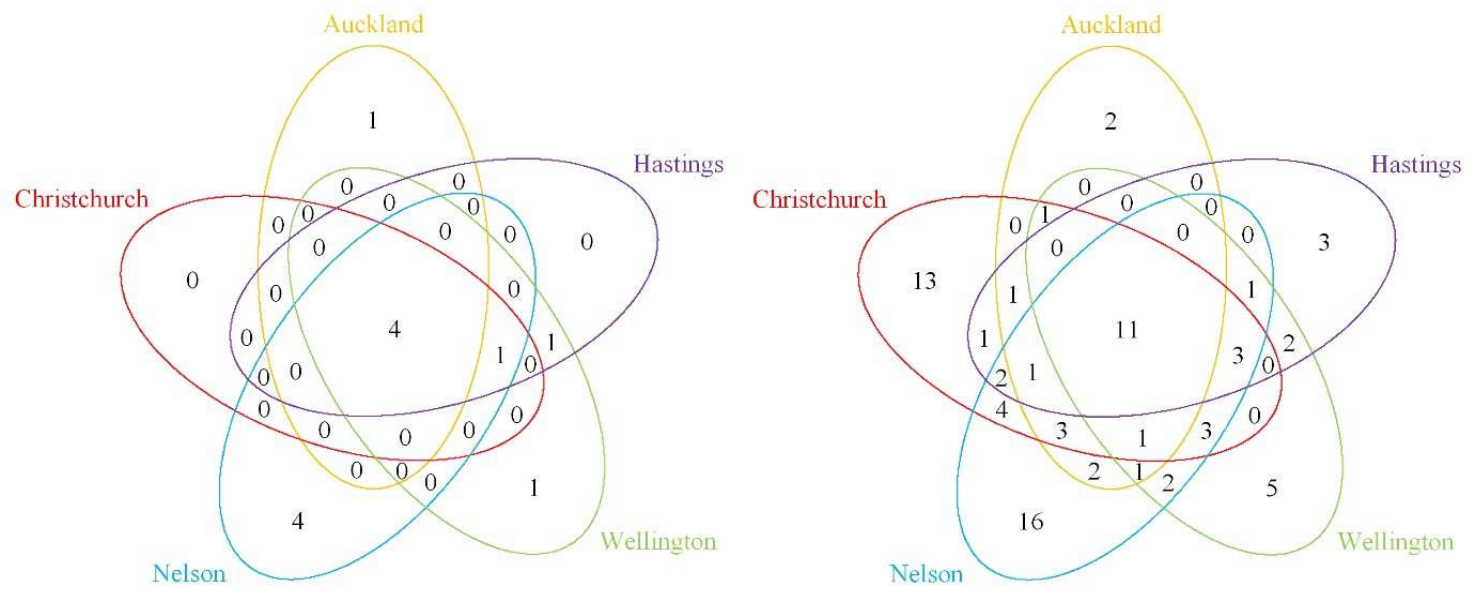

Figure 2.9: Venn diagrams of unique and shared bacteria present in Argentine ants in five cities in New Zealand: a) at the phylum level, and b) at the genus level. Only the presence and absence of bacteria defined to the phylum / genera level were considered.

Proteobacteria and Firmicutes were the phyla present with the higher relative abundance in all cities and were the only non-rare phyla. Actinobacteria and Bacteroidetes were also present in all cities, and were always present $<1 \%$. A g-test was undertaken between Auckland, Wellington, Nelson and Christchurch to test the difference in relative abundance of each bacteria phylum. Hastings was not included as only one sample represented the city. Samples were rarefied to 2,000 reads (the lowest number of read in one sample) each to allow comparison between cities. Bonferroni corrected p-values indicated significant differences between the four cities of interest for two phyla: Firmicutes and Proteobacteria (Table 2.5).

Of the 78 identified genera in New Zealand, 11 bacteria genera were shared between all five cities (Figure 2.9 and Table 2.6). 
Table 2.6: Bacterial genera present in Argentine ants in New Zealand. Only genera present in all New Zealand cities are presented. The relative abundance mean of genera is presented in percentage and the mean of raw number of reads identified for each phylum is in brackets. For Hastings, no means were calculated as only one sample represented the city; the relative abundance and the number of reads corresponded to the data from that single sample. Genera present $>1 \%$ at least in one city are indicated by $\wedge$, and genera present $>1 \%$ in all cities are in bold. Relative abundances $>1 \%$ are in bold. In order to compare bacteria presence between cities, g-tests were realized on the relative abundance and on the number of reads of different genera after rarefaction to 2,000 reads. Bonferroni corrected p-values are presented for each phylum first for the relative abundance and in brackets for the number of reads. Significant p-values are indicated by a *. Hastings was not included in the g-tests as the city was only represented by one sample. Non-classified OTUs and reads ("Unclassified" and "Other phyla") were marginal $(<0.05 \%)$ in all cities. Lactobacillus and Gluconobacter were the only two genera present $>1 \%$ in all cities. No other genera were present $>1 \%$ even in only one city. The number of reads differs significantly between the cities for Lactobacillus and Gluconobacter.

\begin{tabular}{|c|c|c|c|c|c|c|c|}
\hline Phylum & Genus & Auckland & Hastings & $\begin{array}{c}\text { City } \\
\text { Wellington }\end{array}$ & Nelson & Christchurch & $\begin{array}{c}\text { Bonferroni } \\
\text { corrected } \\
\text { p-value }\end{array}$ \\
\hline Firmicutes & Lactobacillus $^{\wedge}$ & $\begin{array}{l}\mathbf{2 8 . 4 9} \\
(1376)\end{array}$ & $\mathbf{3 4 . 1 0}(2820)$ & 14.99 (1272) & $6.65(558)$ & 14.01 (1124) & $2.24 \mathrm{e}^{-60 *}$ \\
\hline \multirow[t]{13}{*}{ Proteobacteria } & Caulobacter & $0.01(1)$ & $0.02(2)$ & $0.10(5)$ & $0.04(3)$ & $0.07(6)$ & NA \\
\hline & Bradyrhizobium & $0.10(7)$ & $0.15(12)$ & $0.40(22)$ & $0.36(28)$ & $0.52(47)$ & 1 \\
\hline & Ochrobactrum & $0.01(1)$ & $0.02(2)$ & $0.07(3)$ & $0.04(3)$ & 0.14 (13) & NA \\
\hline & Gluconobacter $^{\wedge}$ & $\begin{array}{l}\mathbf{3 4 . 8 7} \\
(2272)\end{array}$ & $44.10(3647)$ & 76.43 (5849) & $\mathbf{6 4 . 1 3}(5076)$ & $75.13(6435)$ & $2.04 \mathrm{e}^{-84 *}$ \\
\hline & Sphingomonas $^{\wedge}$ & $0.01(1)$ & $0.17(14)$ & $0.05(3)$ & $0.22(17)$ & $0.26(23)$ & NA \\
\hline & Achromobacter & $0.03(2)$ & $0.05(4)$ & $0.39(18)$ & $0.08(6)$ & $0.17(15)$ & 1 \\
\hline & Delftia & $0.14(10)$ & $0.21(17)$ & $0.30(15)$ & $0.38(30)$ & $0.64(57)$ & 1 \\
\hline & Acinetobacter & $0.11(8)$ & $0.23(19)$ & $0.14(8)$ & $0.39(30)$ & $0.43(39)$ & 1 \\
\hline & Pseudomonas & $0.12(9)$ & $0.10(8)$ & $0.20(9)$ & $0.20(28)$ & 0.67 (59) & 0.32 \\
\hline & $\begin{array}{l}\text { Stenotrophomona } \\
s\end{array}$ & $0.12(8)$ & $0.12(10)$ & $0.12(6)$ & $0.20(15)$ & $0.38(34)$ & 1 \\
\hline & $\begin{array}{l}\text { Total number of } \\
\text { named OTUs }\end{array}$ & $\begin{array}{l}64.25 \\
(3715)\end{array}$ & 79.59 (6583) & $93.72(7251)$ & 74.23 (5938) & 93.41 (7939) & NA \\
\hline & $\begin{array}{l}\text { OTUs in each } \\
\text { cities }\end{array}$ & NA (6061) & NA (8269) & NA (7720) & NA (7797) & NA (8490) & NA \\
\hline & $\begin{array}{l}\text { Total number only } \\
\text { present in all cities }\end{array}$ & $\begin{array}{c}64.01 \\
(3695)\end{array}$ & 79.27 (6555) & $93.20(7210)$ & 72.85 (5794) & $92.60(7852)$ & NA \\
\hline
\end{tabular}

Only one sample was used for Hastings leading to a possible biase on the low number of genera and phyla identified in this specific city. Gluconobacter (mean $=58.93 \pm 8.35 \%$ ) and Lactobacillus (mean $=19.67 \pm 5.04 \%)$ were the only non-rare bacteria present in all cities (Table 2.6). The relative abundance of the two bacteria differed significantly between cities (Table 2.6).

\subsection{Discussion}

To our knowledge, this is the first study describing the bacterial diversity present in Argentine ants. We confirmed that bacterial diversity is higher in the ant' home range, 
Argentina, than in invaded countries, the United States (primary invasion), Australia (secondary invasion) and New Zealand (tertiary invasion). As predicted, bacterial diversity decreased from the ant home range to invaded countries as the number of invasion steps increase along the invasion pathway. Bacterial diversity can be observed within countries like in New Zealand. In addition, bacteria genera including bacteria known for their beneficial effects on their insect hosts were detected in Argentine ants. An Argentine ant core bacterial composition is proposed.

\section{Decrease of bacterial diversity along the invasion pathway}

Under the enemy release hypothesis (ERH), invasive species leave most of their natural enemies including pathogens in their home range. This loss of enemies eventually promote the invasive species spread in their novel environment (Roy et al., 2011). Part of the ERH seems fulfilled in Argentine ants. Indeed, bacterial diversity present in Argentine ants is higher in the ant home range, Argentina, than in invaded countries, the United States, Australia and New Zealand. This loss of pathogens during invasion has also been reported in other species such as invasive shrimps (Dikerogammarus villosus), sparrows (Passer domesticus) or fishes (Silurus glanis, Lepomis gibbosus) (Marzal et al., 2011; Arundell et al., 2014; Sheath et al., 2015). Loss of pathogens during the invasion process has been described in other ant species too. The red imported fire ant (Solenopsis invicta) presents a lower number of infections in their invaded range than in their home range in South America (Yang et al., 2010).

The loss of bacterial infections in Argentine ants may have benefited the ants and promoted their expansion in their new environments, as Argentine ants are present in large populations in their invasive range. The low infection diversity and the loss of pathogenic enemies in Argentine ants may lower the energy allocated to both individual and social immunity. On an individual level, the invasive ant immune system may require less energy to produce protective compounds, and the ants may spend less time and energy selfgrooming. On the colony level, the ants may spend less time allo-grooming. As a trade-off, invasive ants may in consequence possess more time and energy to collect new food for the colony, increase the number of available foragers, and successfully defend food sources against other competitors. This situation may increase successful foraging by the ants in their invasive range. However, to fully accept the enemy released hypothesis (Roy et al., 
2011), further analyses need to highlight the correlation between the decrease of bacterial diversity and a higher fitness and larger populations of Argentine ants in their invaded range than in their home range .

Bacterial diversity decreased with the number of invasion steps as predicted. Argentine ants from the ant home range Argentina and primary invaded country the United States have higher bacterial diversity than Argentine ants from secondary and tertiary invaded countries, Australia and New Zealand respectively. The Argentine ant population underwent multiple bottlenecks along their invasion pathway from Argentina to New Zealand (Suarez et al., 1999; Suhr et al., 2011). At the same time, the bacterial diversity associated with the ant underwent multiple bottlenecks, explaining the observed bacterial diversity decrease. Similarly, the red imported fire ants present less bacteria in their invasive range than in their home range (Yang et al., 2010). More specifically the number of pathogens present in the red imported fire ant invasive range seems to decrease with the number of invasion steps. The red imported fire ants were introduced multiple times from South America to the United States. Then the ants were transported from the United States to other countries, including China, Taiwan, Australia and New Zealand (Ascunce et al., 2011). Out of the six pathogens screened by Yang et al. (2010), only Solenopsis invicta virus -1 and -2 (respectively SINV-1 and SINV-2) were found in the United States and Taiwan, and only SINV-1 in China. No pathogens were detected in Australia and New Zealand. For Argentine ants in New Zealand, the invasive ants were transported from Argentina, to Europe, then to Australia and finally New Zealand (Corin et al., 2007a, 2007b; Suhr et al., 2011). Therefore additional sampling from invaded sites in Europe and Australia may complete and reinforce the results of this study on the decrease of bacterial diversity in Argentine ants following invasion pathway with the number of invasion steps. In addition, Argentine ants from Argentina and the United States present a higher number of unique bacterial genera compared to Australia and New Zealand. This reduced diversity may be due to the difference of time the ants evolved in their habitats, the geographical range and the diversity of the habitats (Flory and Clay, 2013). Argentine ants were first detected in the United States in the early 1900's while it was only later detected in Australia and New Zealand, 1931 and 1990 respectively (Wetterer et al., 2009). Argentine ants had less time in Australia and New Zealand to accumulate pathogens compared the United States. Following the pathogen accumulation theory, Argentine ant should present a higher bacterial diversity and higher number of rare bacteria in Australia than in New Zealand. 
While the number of non-rare bacteria, bacteria present at least at $1 \%$ in relative abundance, was similar between the two countries, ants from New Zealand presented a higher number of rare bacterial genera, genera present $>1 \%$ in a sample. This result may be caused by the disparity in the number of samples collected and evaluated between the two countries. Only one sample from one city from Australia was available for analysis, while I was able to collect samples from five cities in New Zealand. Further sampling from sites in Australia would offer better support on the bacterial diversity present in the country and especially on the number of rare genera.

\section{Argentine ant core bacteria maintained during the invasion process}

The successful spread of Argentine ants in their new environments suggests that the decrease of bacterial diversity in the ants did not negatively affect the ants. Negative effects from the loss of favourable symbionts in the ant may have been lower than the benefits conferred by the loss of pathogenic bacteria. However, the preservation of core bacteria in Argentine ants may have been fundamental for the ant survival, immunity, reproduction, development and spread. The core bacteria of Argentine ants was defined in this study as the bacteria present in all Argentine ant samples, in each city of the country investigated. Under this definition, nine bacterial genera from two phyla constitute the core of bacterial genera present in Argentine ants: Lactobacillus and Gluconobacter, and at a lesser extend Caulobacter, Bradyrhizobium, Achromobacter, Delfia, Acinetobacter, Pseudomonas and Stenotrophomonas. All bacterial genera are bacteria from the Proteobacteria phylum but for Lactobacillus which is from the Firmicutes phylum.

Lactobacillus and Gluconobacter dominate the core of the bacteria present in Argentine ants. The two bacteria genera are the most represented genera in Argentine ants in all countries. These results indicate a potential important beneficial relationship between Argentine ants and these two genera. Lactobacillus is a known genus of favourable facultative symbionts, especially studied in bees (Apis spp.) and bumblebees (Bumbus spp.) (Corby-Harris et al., 2014; Praet et al., 2015). Some bacteria from the genus ares part of the core bacteria of bees and bumble bees. These favourable associates produce antimicrobial compounds promoting their bee and bumble bee host health (Forsgren et al., 2009; Yoshiyama and Kimura, 2009). The core bacteria are acquired horizontally by workers from other nestmates or through ingestion of the colony honey. They are 
maintained in the colony where they enhance colony immunity (Vásquez et al., 2012). Lactobacillus bacteria are also found at high abundance in the fungus growing ants Mycocepurus smithii (Kellner et al., 2015). The Lactobacillus sp. bacterium offers protection from pathogenic fungal and bacterial infections to both the ant fungus garden and the ant themselves (Kellner et al., 2015). The bacterium is hypothetically acquired by the ants in their environment (Kellner et al., 2015). Similarly, bacteria from the Gluconobacter genus can be largely found in the guts of bees (Apis spp.) as facultative symbionts (Anderson et al., 2011; Martinson et al., 2011). Some Gluconobacter species have however been identified as pathogenic in flies (Drosophila spp.), inducing chronicle gut inflammation in their hosts (Matos and Leulier, 2014). The large proportion of Gluconobacter in all Argentine ants may indicate a beneficial relationship between the ants and the bacterial genus as with bees instead of a detrimental one as with flies. Bacteria from the genera Lactobacillus and Gluconobacter may participate to the Argentine ant immune system, preventing pathogenic infections in individuals and in the colonies.

The other bacterial genera part of Argentine ant core bacteria, Caulobacter, Bradyrhizobium, Achromobacter, Delfia, Acinetobacter, Pseudomonas and Stenotrophomonas, present a variety of proprieties such lignocellulose degradation in termite guts (Anoplophora glabripennis) (Scully et al., 2013), possible denitrification proprieties in beetles (Thorectes lusitanicus) (Hernández et al., 2013), phosphate solubisation in plants (Jha and Saraf, 2015). While these bacteria can be found in ants (Li et al., 2005; Stoll et al., 2007; Suen et al., 2010; Ishak et al., 2011b; Kellner et al., 2015; Liberti et al., 2015), their relationship with their hosts is not yet known. Their presence in all Argentine ants may indicate either a beneficial interaction or a non-harmful one. It is also possible that these genera are common bacterial genera in the ant environment. Argentine ants may then easily acquire bacteria from these genera by contact with soil or food sources.

Core bacteria may have been essential to the successful spread of Argentine ants in their novel environments. Their presence in all sampled Argentine ants may be explained by two non-exclusive hypothesises. Firstly, Argentine ant queens may harbour Lactobacillus, Gluconobacter, Caulobacter, Bradyrhizobium, Achromobacter, Delfia, Acinetobacter, Pseudomonas and Stenotrophomonas in their home range in Argentina. Infected queens 
may have been transported to new invasive range. The genera may after be maintained in the ant colony by horizontal transfer via trophallaxis exchanges between the queens and their workers as well as exchanges among workers, or by vertical transmission from the queens to their offspring. Secondly, if the nine genera are largely present in the ant environment, workers may have acquired bacteria from these genera when foraging and maintained them in their colonies by trophallaxis. Further analysis of bacteria presence in Argentine ant queens and bacteria strains will offer insights in possible differences in bacteria community between queens and workers as previously observed in bees. This may also inform on the acquisition path of these core bacteria and a better understanding in the transmission of favourable bacteria in Argentine ants.

\section{Loss of Wolbachia and Rickettsia during the invasion process}

Two remarkable bacterial genera (i.e Wolbachia and Rickettsia) are among the bacterial genera that appear to have been lost during the invasion process. Only samples from Argentina presented Wolbachia infections. This result concurs with previous studies recording Wolbachia infections in Argentine ants throughout the ant home range and one invaded area Hawaii (Tsutsui et al., 2003; Reuter et al., 2005). No infections by Wolbachia in Argentine ants has been reported outside Argentina except in Hawaii (Tsutsui et al., 2003; Reuter et al., 2005). My samples from Hawaii did not present any infection. This absence may be due to the marginal presence of the endosymbiont in the ants and my limited sampling. Rickettsia was found in both Argentina and the United States, making these results the first report of the presence of Rickettsia in Argentine ants. Wolbachia and Rickettsia are a facultative endosymbionts, rarely obligatory, transmitted both vertically and horizontally (Viljakainen et al., 2008; Weinert et al., 2009), that can benefit its arthropod host greatly (Braquart-Varnier et al., 2015). Wolbachia is found in many species including up to 66\% of arthropod species (Hilgenboecker et al., 2008). Ants (such as Formica rufa, Formica cinerea and Anoplolepis gracilipes), wasps and flies (Drosophila spp.) are just a few of the many arthropods infected by the genus of bacteria (Viljakainen et al., 2008; Sirviö and Pamilo, 2010; Sebastien et al., 2011; Ahmed et al., 2013; Vale and Jardine, 2015). Wolbachia sp. can offer some of its fly and mosquito hosts protection against viral infection (Rainey et al., 2014), it may decrease virus transmission rates from

mosquitos to mosquitos' preys (Touret et al., 2014), and increases its host basal immunity and life span (Bian et al., 2010; Rainey et al., 2014; Becerra et al., 2015; Raquin et al., 
2015). For its part, Rickettsia sp. can be found among other ant species (Anoplolepis gracilipes), wasps (Megastigmus amicorum and M. bipunctatus), bees (Halictus sp., Lasioglossum sp. And Osmia bicornis) (Sebastien et al., 2011; Paulson et al., 2014; Gerth et al., 2015). Bacteria from this genus can increase the fecundity and survival of their fly hosts (Jiggins and Hurst, 2011; Asiimwe et al., 2014).

While Wolbachia can be found in $22 \%$ of ant species (Kautz et al., 2013a) in all ant casts, including in Argentine ants (Tsutsui et al., 2003), no information about the prevalence of Rickettsia in ant species are available. Furthermore few examples of the known bacteria effects on other species have been tested and described in ants. No cytoplasmic incompatibility, male killing, sex-ratio modification or fitness effects were observed in ant colonies presenting infections by Wolbachia sp. (Wenseleers et al., 2002; Bouwma and Shoemaker, 2011). Though in Formica truncorm, Wolbachia sp. infected colonies produced more sexual individuals than non-infected colonies (Wenseleers et al., 2002). The full effects of Wolbachia and Rickettsia in Argentine ants are therefore unknown. It has been postulated that the bacteria from these genera may protect the ants from pathogenic infections and influence reproduction. The loss of Wolbachia and Rickettsia during the ant invasion process does not seem to have stopped the spread and settlement of the Argentine ants in new environments. Nevertheless, the loss of bacteria from these two genera may have affected the long term survival of the ants in New Zealand.

\section{Acquired bacteria in the Argentine ant invasive range}

Among the non-rare bacterial genera meaning bacteria present $<1 \%$ in relative abundance in the samples, only Weissella and Stenotrophonas genera were gained by the ants, and gained only in the United States. Weissella bacteria are generally associated with fermentation (Tajabadi et al., 2012). In bees, the bacteria from these genus prevent pathogenic infections by activating their host immune system (Yoshiyama et al., 2013). Stenotrophomonas bacteria are found in multiple ant species (Baird et al., 2007; He et al., 2014; Kellner et al., 2015); however their role has not yet been characterised. It is possible that these two bacterial genera were acquired by Argentine ants in the United States, and their effects are not understood currently. While only two non-rare bacterial genera were acquired in the invasion process, a higher number of rare bacterial genera were detected in Argentine ants in Argentina and the United States. The low number of acquired bacterial 
genera in New Zealand compared to the United States may be due to the invasive history of the ants in the two countries. Argentine ants were present in the United States since the early 1900's and was only first detected in New Zealand in 1990 (Wetterer et al., 2009). As previously discussed, the accumulation of new bacteria in invasive species is correlated with the time spent in new environments as well as the geographical range and the diversity of the habitats (Flory and Clay, 2013). Argentine ants in New Zealand had less time to accumulate new bacteria than Argentine ants in the United States. In addition, the effect of primary versus secondary invasion process between the two countries cannot be fully disregarded.

Argentine ants did conserve their core bacteria while losing some bacterial infections during the invasion process. The conservation of some bacteria genera and simultaneous loss of others during invasion has been observed in other invasive species. For example, invasive citrus thrips (Pezothrips kellyanus) loss their Wolbachia endosymbionts while conserving their association with Cardinium when invading New Zealand and the Mediterranean region from their home range in Australia (Nguyen et al., 2015).

\section{Bacterial diversity within New Zealand}

Bacterial diversity in New Zealand was not significantly different between cities. Argentine ants from the South Island seem to present a bacterial diversity non-significantly higher than ants from the North Island. Surprisingly bacterial diversity in Argentine ants seems to have marginally increased from their entry in New Zealand in the North Island in Auckland to their spread to the South Island. Argentine ants may be in contact with a higher diversity of bacteria in the South Island than in the North Island. The two islands present differences in biodiversity and climate (Saunders and Norton, 2001; Cooling et al., 2012). It is therefore possible that the islands also present differences in bacterial diversity. This overall bacterial diversity difference in the islands may explain the differences in bacteria present in Argentine ants in New Zealand. In addition, this result concords with the observed variability of bacterial diversity present in Argentine ants in Argentina and the United States (Figure 2.6). Argentine ants may present a specific core of bacteria while still being susceptible to horizontal transfer of bacteria from their environment. For example, the alfalfa weevil (Hypera postica) gained Wolbachia in their invasive range (Iwase et al., 2015). 
Argentine ants present overall a low bacterial diversity in New Zealand. This low bacterial diversity associated with a low genetic diversity (Corin et al., 2007a) may lead to a low resistance of Argentine ants against new infections. In bees (Apis spp.), high bacterial diversity has been associated with high genetic diversity and healthier individuals (Mattila et al., 2012). The ants may consequently have been susceptible to pathogenic infections. The population decline of Argentine ants in New Zealand (Cooling et al., 2012) may have been caused by pathogenic micro-organisms present in the country or facilitated by the reduced presence of beneficial bacteria.

\section{Critical evaluation of experimental procedures}

The difference in sampling time between countries may have affected the results. Samples from Argentina were from older collections (the oldest samples dated from 1999) while samples from the United States, Australia and New Zealand were collected more recently (between 2011 and 2013). While the samples were conserved in $70-100 \%$ ethanol at $-20^{\circ} \mathrm{C}$, DNA quality may have degraded over time. The genetic material degradation may have been enough for the bacterial DNA to be of a quality too poor to be correctly amplified by the $16 \mathrm{~S}$ primers. However, despite the samples being old, bacterial diversity was found to be higher in Argentina than in the other countries. The older samples, may raise the question of diversity over-estimation due to bacterial DNA degradation. Reduced DNA degradation could have allowed the successful amplification of bacterial DNA by the $16 \mathrm{~S}$ primers but could have led to the mis-amplification of some bacterial DNA, and eventually to the misidentification of the amplified sequences. This hypothesis is unlikely. If the 16S gene DNA degraded in the samples, two possibilities may occur. Firstly, the degraded sequences may still hold a strong identity match to known bacteria genera, above the $97 \%$ identity threshold. The sequences would then be assigned to the corresponding OTUs, and then the correct bacterial identity. Secondly, the sequences may have degraded so much that they could not be identified. This would lead to a high number of non-assigned bacteria. However, the relative abundance of non-assigned OTUs was similar between countries, and therefore between old and new samples. These results indicate that degradation of bacteria DNA in old samples was negligible. 
Argentine ants present a lower bacterial diversity in their invasive range than in their home range. Bacteria loss occurred along the invasion pathway and reflect the ant invasion history. The ants did conserve a core of bacterial genera including Lactobacillus and Gluconobacter, two genera with known beneficial species. The lowest bacterial diversity was found in New Zealand. This low bacterial diversity associated with a low genetic diversity of Argentine ants may have reduced the ant resistance against pathogenic infections and may explain the ant large scale population decline observed in New Zealand. Invasive species may be released from their natural enemies but still be susceptible to new infections. Bacterial diversity decline may affect Argentine ant survival and population dynamics. Antibiotic treatments may be used in order to artificially decrease bacterial diversity in Argentine ants and to understand the consequences of bacterial diversity decrease on Argentine ant survival and fitness (see Chapter 3). 
Chapter 3. Bacterial diversity decline in Argentine ants after antibiotic treatments 


\subsection{Abstract}

Invasive populations may fluctuate over time. Some invasive population declines have specifically been associated with pathogenic infections. Investigating the effects of bacterial diversity changes via the use of antibiotics and the consequences of bacterial diversity changes on invasive species fitness may offer a better understanding in these population declines. Argentine ants are a successful widespread invasive species in New Zealand. Part of the invasive ant population inexplicably declined in New Zealand, and pathogenic infections were hypothesised as the cause. To induce an artificial change in bacterial diversity, Argentine ant colonies were treated in the laboratory with antibiotics, ampicillin and gentamicin. The change in bacterial diversity and relative abundance of each genus after antibiotic treatments was characterized using 454 sequencing. Ampicillin specifically increased the abundance of Lactobacillus and Sphingomonas, and decreased the abundance of Achromobacter. Gentamicin increased the abundance of Delfia and decreased the abundance of Gluconobacter. Only ampicillin decreased Argentine ant survival, and neither antibiotic affected brood production. The increase in bacterial diversity and change in relative abundance may be at the origin of the decrease of the ant survival after antibiotic treatments. Further analyses are needed to determine which specific bacteria may have been influenced by antibiotic treatments. The absence of effects of antibiotic treatments on ant fitness may be due to a low exposition of the queens to antibiotics. New experiments directly treating the queens with antibiotics may decrease the egg production by the queens, and consequently decrease the colony fitness. Bacteria are still possible candidates to explain the observed Argentine ant population decline, and may be involved in other invasive species population dynamics. 


\subsection{Introduction}

\section{Pathogenic infections in population dynamics}

Populations fluctuate throughout time. Declines may follow population booms during a dynamic cycle or may occur as exceptional events. For example, the invasive yellow crazy ant (Anoplolepis gracilipes) populations seem to follow a boom and bust pattern in Australia and Tokelau (Gruber et al., 2012; Cooling and Hoffmann, 2015). Population declines may be attributed to some factors such as limited food sources, limited space availability, and pathogenic infections. Pathogenic infections correspond to infections by micro-organisms such as bacteria and viruses that cause diseases in their hosts. In honey bees (Apis mellifera), the pathogenic bacterial American foulbrood agent (Paenibacillus larvae) can cause the death of infected larvae (Genersch, 2010a). The infected colonies are weakened, and at length may die. The highly pathogenic Squirrel pox virus (Squirrel parapoxvirus) caused the dramatic population decline of grey squirrel (Scurius carolinensus), after the virus introduction in the grey squirrel native range (Strauss et al., 2012).

\section{Favourable bacterial associations}

Social insects are the hosts to numerous pathogen infections including bacteria (Forsgren, 2010; Genersch, 2010), viruses (Highfield et al., 2009; Valles et al., 2009), and fungi (Genersch, 2010; Evans et al., 2011). However, social insects present multiple defences against harmful infections. On the individual level, self-grooming behaviour and individual immune system response help workers maintain their own health, while on the colony level allo-grooming (Okuno et al., 2011; Qiu et al., 2014) and hygienic behaviour (i.e. the disposal of nest-mate corpses) (Spivak and REuter, 2001; Diez et al., 2014) can provide protection against the spread of infections in nests. In addition to their individual and social immunity, social insects can limit the proliferation of pathogens via antimicrobial and antifungal activity compounds present in specific food (Gherman et al., 2014), in the resin foraged and placed in their nesting environment (Chapuisat et al., 2007; Brütsch and Chapuisat, 2014; Drescher et al., 2014) or in their own excrements (Koch and SchmidHempel, 2011; Chouvenc et al., 2013). Furthermore, social insects can be intimately associated with symbiotic favourable bacteria producing antibiotic and antifungal 
compounds, such as Lactobacillus (Valmir Santos et al., 2004; Forsgren et al., 2009; Yoshiyama and Kimura, 2009; Mattoso et al., 2012; Koehler et al., 2013). Hence, the bacterial diversity associated with social insects has been associated with their health and wellbeing (Koch and Schmid-Hempel, 2011; Poulsen and Sapountzis, 2012; Vásquez et al., 2012). The loss of these favourable associates may affect host populations and lead to population declines.

\section{Antibiotic treatments}

Antibiotic treatments are used in order to understand the specific role of bacteria association with their hosts. For example, Pseudomonas spp. are part of the coffee berry borers (Hypothenemus hampei) gut bacteria, and can be killed by tetracycline, rifampicin and streptomycin treatments. After antibiotic treatments, coffee berry borers were unable to degrade the caffeine present in their food source, indicating the major role of Pseudomas spp. in their host digestion (Ceja-Navarro et al., 2015). Following rifampin treatments, the role of the bacterial genus Blochmannia in carpenter ants (Camponotus fellah) host was highlighted (de Souza et al., 2009). The bacteria are involved in their host colony growth and immunity system. Antibiotic treatments may therefore highlight major bacterial associations with their hosts, and in their host population dynamics.

\section{The case of the invasive Argentine ant}

Argentine ants (Linepithema humile) were introduced in New Zealand after a complex invasion path (see Chapter 1, section 1.8). In addition to a low genetic diversity, the ants seem to present a lower diversity of bacterial genera in New Zealand than in their home range (see Chapter 2). Argentines ants may have lost valuable associations with protective bacteria along their invasion pathway, lowering their immune system. Bacterial infections in Argentine ants in New Zealand may therefore be responsible for the observed population decline. The loss of these infections through antibiotic treatments may improve Argentine ant fitness. 


\section{Chapter aims}

In this chapter, 454 sequencing was used to characterise changes to the bacterial diversity after antibiotic treatments, as a preliminary step to understand the role of bacteria in Argentine ant population dynamics. It was hypothesised that: 1) antibiotic treatments will decrease bacterial diversity in Argentine ants; and 2) the loss of pathogenic bacteria will increase Argentine ant fitness.

\subsection{Materials and methods}

\subsubsection{Colony collection and laboratory rearing conditions}

To test the effect of the loss of specific pathogens on long term Argentine ant survival and colony fitness, multiple nests were collected in a private garden in Hastings (North Island, New Zealand - latitude -39.628287, longitude 176.858909), at the end of February 2012. Argentine ants form a super colony in New Zealand (Corin et al., 2007a). This characteristic allowed me to place the nests together into one colony. The resulting colony contained 46 queens, over 3,000 workers and some brood. The ants were placed in a large plastic container $(20.5 \times 14.0 \times 7.0 \mathrm{~cm})$ during three months for acclimation. The container walls were coated with fluon ${ }^{\mathrm{TM}}$ (Polytetrafluoroethylene PTFE-30; BioQuip Products, Inc.) to prevent any escape. The lid had an opening $(4.0 \times 9.0 \mathrm{~cm})$ in the middle covered by a tight mesh net allowing air to pass through. Artificial nests consisted of three $10 \mathrm{~mL}$ nest tubes, filled up with water to one third and plugged with a cotton wool to provide a humid environment. Red cellophane was used to shelter the ant nests from light and mimic light conditions in natural nests. The ants were kept in the laboratory at $\sim 20^{\circ} \mathrm{C}, \sim 60 \%$ relative humidity, under a 16-hour day / 8-hour night regime. This environment was similar throughout the different stages of the experiment. The ants were fed twice a week with fresh water, $20 \%(\mathrm{v} / \mathrm{v})$ honey water mix and three $0.3 \mathrm{~cm}$ long pieces of freshly killed mealworms (Tenebrio molitor larvae).

\subsubsection{Antibiotic treatments}

\section{Antibiotics}

To study the bacterial diversity decrease in Argentine ants, four antibiotics (i.e. ampicillin, gentamicin, kanamycin, and spectinomycin) were selected. The antibiotics were selected 
for their effectiveness on the various bacterial spectra and different targets within bacteria, and therefore their potential different effects on bacterial diversity present in Argentine ants. Ampicillin is a semi synthetic $\beta$-lactam with bactericide proprieties. The antibiotic inhibits the transpeptidase enzyme needed in cell wall synthesis, affecting both Gram positive and Gram negative bacteria. Gentamicin, kanamycin and spectinomycin are natural aminoglycosides with bactericide proprieties. These antibiotics interfere with protein synthesis by binding with $30 \mathrm{~S}$ ribosome subunit of Gram negative bacteria and of a few Gram positive bacteria.

\section{Optimal antibiotic concentration}

Antibiotic concentrations for the treatments were optimised prior to the experiments. An optimal antibiotic concentration was defined as high enough to modify bacterial diversity without inducing immediate death of the ants. Three different antibiotic concentrations $(1.00 \mathrm{mg} / \mathrm{mL}, 2.50 \mathrm{mg} / \mathrm{mL}$, and $5.00 \mathrm{mg} / \mathrm{mL})$ were tested for each of the four selected antibiotics, ampicillin (Sigma Aldrich), gentamicin (Sigma Aldrich), kanamycin (Sigma Aldrich) and spectinomycin (Sigma Aldrich). A treatment corresponded to one concentration of one antibiotic, $1.00 \mathrm{mg} / \mathrm{mL}$ of ampicillin for example. The control treatment corresponded to the absence of antibiotics in the sugar mix feeding solution (as described later). Each treatment including the control treatment was tested on two groups of 10 workers. Workers were retrieved from the large colony fragment one day prior to treatment. Test groups were conserved in small plastic containers $(9.0 \times 13.0 \times 8.5 \mathrm{~cm})$. The container walls were coated with Fluon ${ }^{\mathrm{TM}}$ (Polytetrafluoroethylene PTFE-30; BioQuip Products, Inc.) to prevent any escape. The lid had an opening $(4.0 \times 3.0 \mathrm{~cm})$ in the middle covered by a tight mesh net allowing air to pass through. One artificial nest tube was placed in each container. Artificial nests consisted of $2 \mathrm{~mL}$ nest tubes $(4.0 \mathrm{~cm}$ long x $1.0 \mathrm{~cm})$ with a small cotton ball soaked in water for humidity. Red cellophane was used to shelter the ant nests from light and mimic light conditions in natural nests. The optimal antibiotic concentration experiment was tested over ten consecutive days. Each group of workers were treated with antibiotics once a day and fed following one treatment for five treatment days. For antibiotic treatments, the groups of workers were fed one $0.50 \mathrm{~mL}$ drop mix of $20 \%$ sucrose (w/v, final concentration) and the appropriate concentration of antibiotic (i.e. $1.00 \mathrm{mg} / \mathrm{mL}, 2.50 \mathrm{mg} / \mathrm{mL}$, or $5.00 \mathrm{mg} / \mathrm{mL}$, final concentration), placed on a tin foil square $\left(\sim 1.5 \mathrm{~cm}^{2}\right)$. Controls were fed $0.50 \mathrm{~mL}$ of $20 \%$ sucrose water $(\mathrm{w} / \mathrm{v})$. After feeding, ants were observed for one hour to ensure that at least one ant fed on the solution mix. After 
five days of antibiotic treatments, all groups were given one $0.5 \mathrm{~cm}$ long piece of freshly killed mealworms and one $0.50 \mathrm{~mL}$ drop of $20 \%$ sucrose water mix (w/v). During the next four consecutive days, the ants were all fed one $0.50 \mathrm{~mL}$ drop of $20 \%$ sucrose water mix $(\mathrm{w} / \mathrm{v})$. The number of ants in each of the different groups were recorded over the 10 days of the trial.

\section{Ampicillin and gentamicin treatments}

Based on the results of the optimal concentration trials later described (see section 3.4.), ampicillin and gentamicin at $5.0 \mathrm{mg} / \mathrm{mL}$ were selected to test the effects of antibiotic on bacterial diversity, and on long-term colony survival and fitness. Each treatment, antibiotics and control, were given to three colonies. Ants were retrieved from the original colony to create nine fragments. Each fragment is considered thereafter as a colony. Each newly created colony was composed of three queens, 300 workers and 15 larvae, and was placed in new containers $(7.0 \times 14.0 \times 20.5 \mathrm{~cm})$ with three artificial nest tubes each as previously described. Colonies were fed twice a week with fresh water, $20 \%(\mathrm{v} / \mathrm{v})$ honey water mix and three $0.3 \mathrm{~cm}$ long pieces of freshly killed mealworms during one month for acclimation. Colonies were not fed for three days prior to starting the antibiotic treatments in order to encourage feeding during treatments. Treatments occurred over 10 days. Colonies were fed $1.00 \mathrm{~mL}$ of a mix composed of $20 \%(\mathrm{w} / \mathrm{v})$ sucrose and $5.00 \mathrm{mg} / \mathrm{mL}$ (final concentration) antibiotics once a day for five consecutive days. Control colonies were feed $1.00 \mathrm{~mL}$ of a mix composed of $20 \%(\mathrm{w} / \mathrm{v})$ sucrose. On the sixth day, all groups were given one $0.5 \mathrm{~cm}$ long piece of freshly killed mealworms and one $1.00 \mathrm{~mL}$ drop of $20 \%(\mathrm{w} / \mathrm{v})$ sucrose water mix. For the next four days, the ants were all fed one $1.00 \mathrm{~mL}$ drop of $20 \%$ (w/v) sucrose water mix. All dead ants in each of the different treatments were counted every day during the treatments. Following the treatments, all colonies were fed twice a week with fresh water, $20 \%(\mathrm{v} / \mathrm{v})$ honey water $\mathrm{mix}$ and three $0.3 \mathrm{~cm}$ long pieces of freshly killed mealworms. After three months, queens, workers, larvae and eggs were counted in each colony.

\subsubsection{Metagenomics sequencing}

\section{Sample collection}

One sample composed of ants freshly collected from the field, before any feeding in the laboratory, and constituted the "before test" group. Additional ants were collected before and after treatments in order to assess the effect of antibiotic treatments on bacterial 
diversity present in ants. Five samples from the antibiotic treatments were collected on the $10^{\text {th }}$ day after the beginning of the treatments. Only six samples from the antibiotic treated ants were analysed with 454 sequencing due to the sequencing plate limitations. Two colonies were randomly selected among the three control colonies, two colonies among the three colonies treated with ampicillin, and one colony among the three colonies threated with gentamicin. All samples were preserved in $100 \%$ ethanol at $-20^{\circ} \mathrm{C}$.

\section{DNA extraction and library creation}

To retrieve bacterial DNA from the samples, DNA was extracted from a pool of 30 ants for each sample. DNA was extracted using a PureLink® Genomic DNA Mini-Kit (Invitrogen) with an additional Phenol-Chloroform purification step (see Chapter 2, section 2.3.1). Bacterial DNA was then amplified using tagged 16S universal primer Gray28F and gray519r and prepared for 454 sequencing (see Chapter 2, section 2.3.1).

\subsubsection{Data Analysis}

Argentine ants survival probability analyses were realised in $\mathrm{R}$ version i386 3.1.3 using the package survival. Comparison between survival probabilities of ants in different treatments was analysed using Cox proportional hazard regression models (Barbieri et al., 2013). The fitness of the colonies (eggs and larva production) were compared by anova based on treatments and number of queens. Significance for all tests was assumed at $p<0.05$.

Sequences from 454 sequencing were analysed using QIIME 1.7.0 (see Chapter 2, section 2.3.2). Changes were made for the rarefaction of samples. Samples were rarefied to 4,000 reads, the lowest number of reads found in a sample to allow comparison between samples. Only one sample represented bacterial diversity before laboratory rearing conditions, and only one sample represented bacterial diversity after gentamicin treatment. Therefore statistical analysis were only undertaken between the control and ampicillin treatments.

\subsection{Results}

\subsubsection{Optimal antibiotic concentration}


Trials to determine the optimal concentration of antibiotics were conducted on small groups of workers using four antibiotics; ampicillin, gentamicin, kanamycin and spectinomycin, at three different concentrations during a 10 day trial (Figure 3.1).

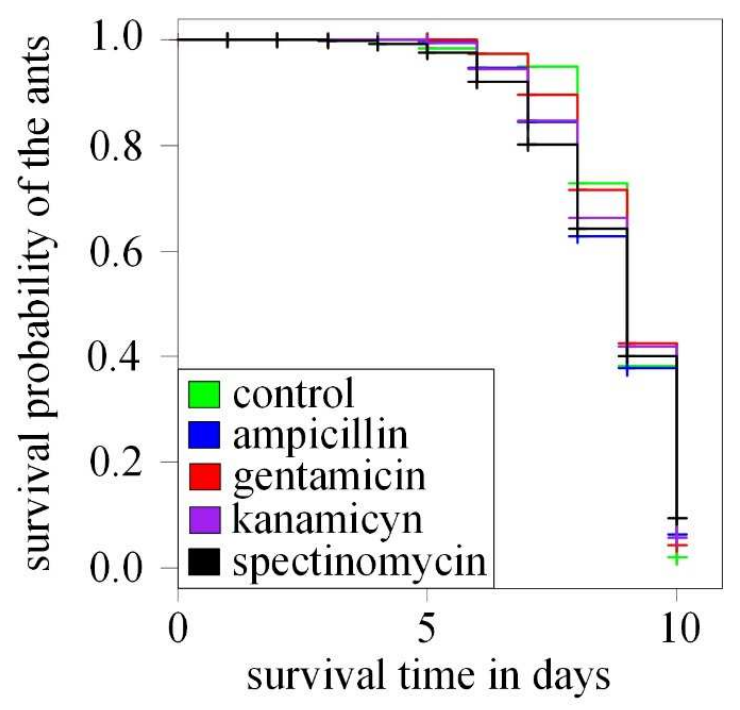

Figure 3.1: Survival probability of Argentine ants during antibiotic treatments with ampicillin, gentamicin, kanamycin and spectinomycin. Each antibiotic treatment survival line corresponds to the mean survival during a 10 day trial of six groups of workers ( $\mathrm{n}=10$ ants), two groups of workers for each of the three different antibiotic concentration treatments. The control treatment was realised on two groups of workers ( $\mathrm{n}=10 \mathrm{ants}$ ). Ants were counted every day.

When pooling the survival results of the three concentrations together for each antibiotic, there was no significant difference in the death rate of ants for any antibiotics compared to the control treatment (Likelihood ratio test $=0.8, \mathrm{df}=4, \mathrm{p}>0.05, \mathrm{n}=2860$; $\mathrm{coxph}$ ).

In order to determine the optimal antibiotic concentration for later experimentations, the different concentrations of antibiotics were compared to each other (Figure 3.2). 

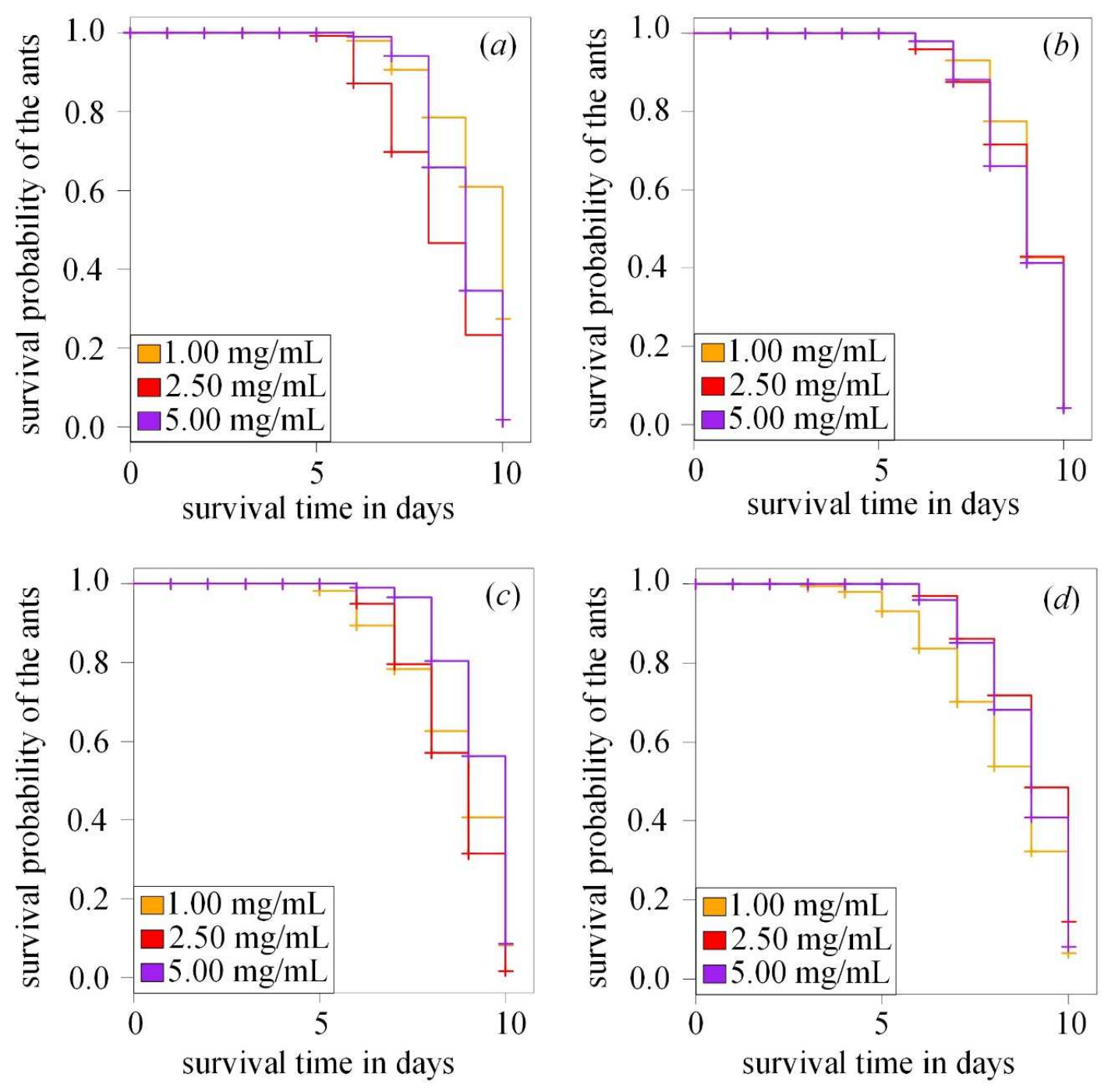

Figure 3.2: Survival probability of Argentine ants during antibiotic treatments at different concentrations for: a) ampicillin; b) gentamicin; c) kanamycin; and d) spectinomycin. Each antibiotic concentration was tested on two groups of workers ( $\mathrm{n}=10$ ants) during a 10 day trial. Ants were counted every day.

There were significant differences between the probability of ant survival at different antibiotic concentrations for ampicillin (Likelihood ratio test $=39.3$, df $=2, p<0.001$, $\mathrm{n}=660 ; \operatorname{coxph}$ ), kanamycin (Likelihood ratio test $=11.2, \mathrm{df}=2, \mathrm{p}<0.05, \mathrm{n}=660 ; \operatorname{coxph}$ ) and spectonmycin (Likelihood ratio test $=8.73, \mathrm{df}=2, \mathrm{p}<0.05, \mathrm{n}=660$; coxph) treatments. No significant differences were observed for gentamicin (Likelihood ratio test $=0.32$, $\mathrm{df}=2, \mathrm{p}>0.05, \mathrm{n}=660$; coxph). In all treatments, ants survived at least four days post 
treatment (Figure 3.2). These results indicated that an antibiotic concentration of $5.00 \mathrm{mg} / \mathrm{mL}$, the highest tested antibiotic concentration, could be used to test the effects of antibiotics on ant fitness without inducing immediate death of the treated ants. Therefore, $5.00 \mathrm{mg} / \mathrm{mL}$ was the concentration used for all following experiments involving antibiotics.

\subsubsection{4 read quality}

Bacterial DNA amplified by 16S primers from Argentine ant samples were collected before laboratory rearing conditions (just after field collection) and after two antibiotic treatments to assess the modification in bacterial diversity after antibiotic treatments.

A total of 273,798 sequence reads was obtained from the three resulting libraries. The libraries contained from 62,897 to 138,672 reads and included reads from 18 extra samples from a different analysis (see Chapter 2). A total of 565 reads were excluded based on their length being either below 200 or above 1,000 nucleotides. A further 261 reads were excluded for their number of ambiguous bases exceeding six nucleotides, 10,118 reads for a mean quality score below 25, and 5,316 reads for presenting a homopolymer run exceeding 6. After these quality controls, a total of 56,845 reads were assigned to the six samples; one sample after field collection and before any feeding in the laboratory, two samples from two control treatment colonies, two samples from two ampicillin treated colonies, and one sample from one gentamicin treated colony. Between 4,258 to 24,386 reads (mean $=9474.14 \pm 3121.96)$ were assigned. A final denoising, chimera and singleton exclusion step with Usearch 7.0.1090 left a total of 54,773 reads. These reads were assigned to the six samples, from 4142 to 23,604 reads per sample (mean $=9,128.83 \pm 3,026.20$ ), and were clustered into 483 Operational taxonomic units (OTUs) with 97\% identity similarity between reads.

\subsubsection{Bacterial diversity between antibiotic treatments}

Alpha diversity was analysed using rarefaction curves based on the number of observed number of operational taxonomic units (OTUs) for each treatment: before laboratory rearing conditions ("before test"), and after treatments for the control, ampicillin and gentamicin treatments (Figure 3.3). 


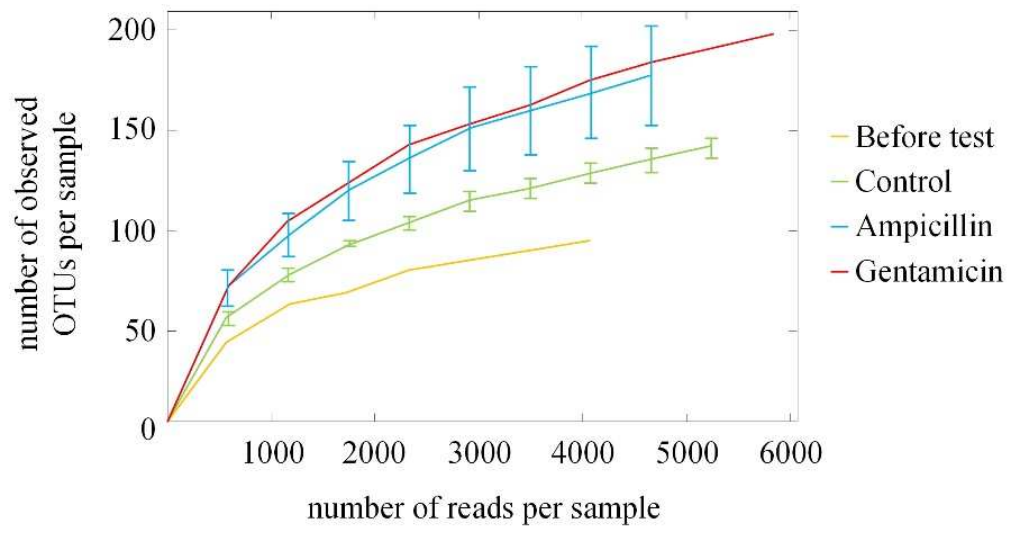

Figure 3.3: Rarefaction curves using QIIME 1.7.0, based on the estimated number of OTUs in Argentine ants before and after antibiotic treatments. No error bars for the standard variation are presented for "Before test" and "Gentamicin" treatments as only one sample was available for each. The length of the rarefaction curve for each treatment is limited by the lowest number of sequence reads in a sample from that treatment.

The number of OTUs observed for each treatment increased with the number of reads. The slopes of the curves slowed to reach plateaux. These results indicate that most of the OTUs diversity could be detected with only 4,000 reads per sample, 4,000 being the lowest number of reads in a sample in this study. The number of OTUs is the lowest before laboratory rearing conditions, before any acclimation and treatments. The highest number of observed OTUs was found after antibiotic treatments. The number of observed OTUs is significantly higher after ampicillin treatment than after the control treatment. Because of the space limits of the 454 sequencing, only one sample for the "before test" and gentamicin treatment could be analysed. Therefore significances for the observed differences involving these two groups cannot be tested. A significant difference can be observed between the control and ampicillin treatment (Figure $3.3^{\circ}$ ). The ampicillin treatment presented a higher number of observed OTUs than the control.

Beta diversity was analysed through principal coordinate analyses (PCoA) to compare bacterial diversity between treatments. PCoA were realised based on weighted and unweighted UniFrac distances between samples after rarefaction to 4,000 reads, under the lowest number of reads in a sample (Figure 3.4). 

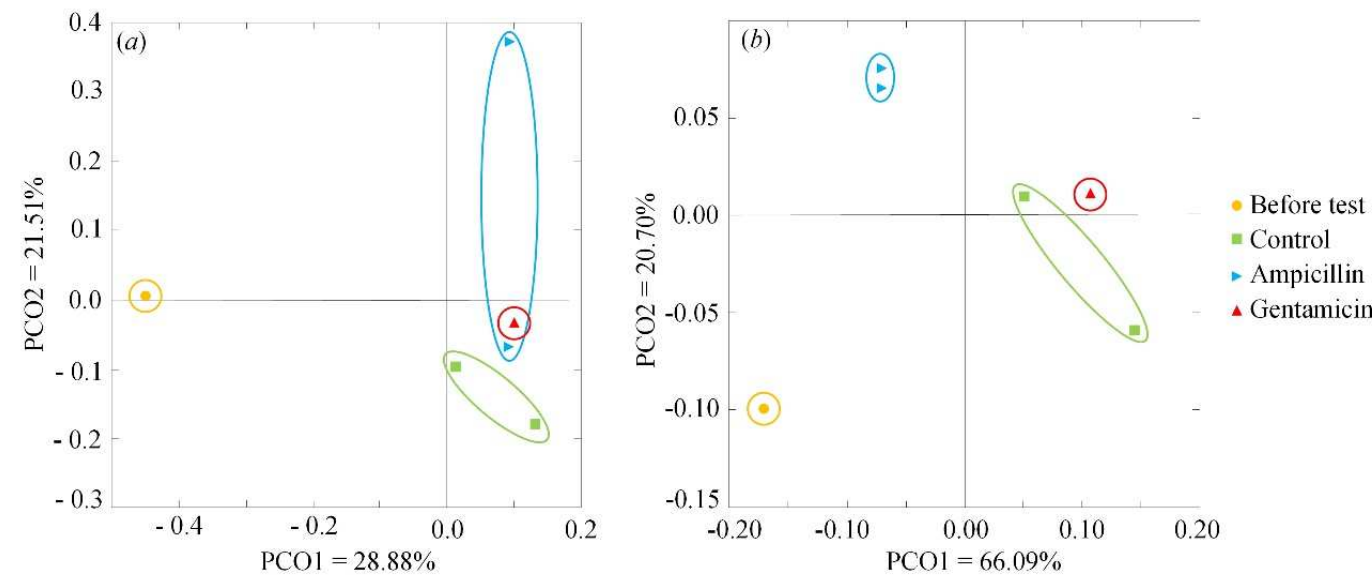

Figure 3.4: Principal coordinate analysis (PCoA) based on a) unweighted and b) weighted phylogenetic UniFrac distances generated in QIIME 1.7.0 of Argentine ant samples before and after antibiotic treatments. Reads were rarefied to 4,000 to allow comparison between samples. Circles were manually added to visualise the dispersion of samples from a same treatment.

Few OTUs were unclassified, meaning outside the bacteria domain or undetermined bacterial phylum $(<0.3 \%)$. In total, 128 bacterial genera belonging to 13 phyla were detected across all treatments. Four phyla and 22 genera were shared by all treatments (Figure 3.5).

(a)

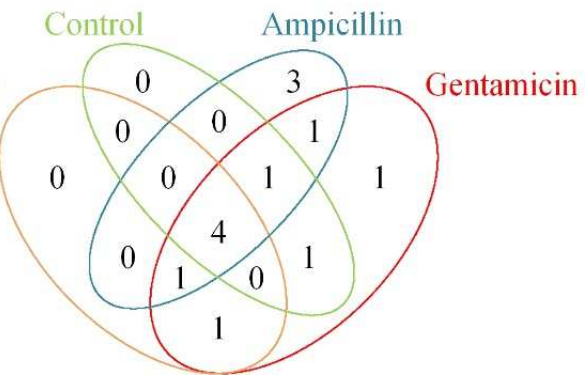

(b)

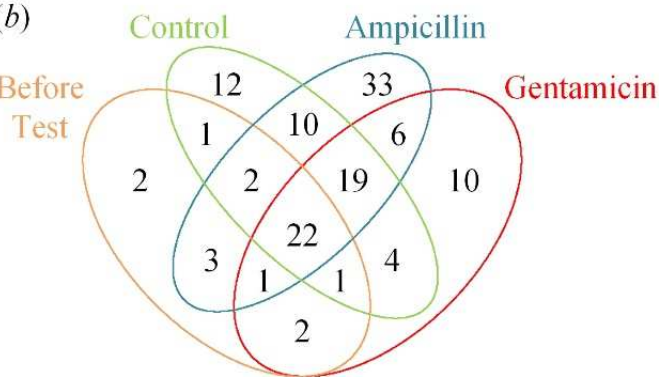

Figure 3.5: Venn diagrams of unique and shared bacteria present in Argentine before and after antibiotic treatments: a) at the phylum level, and b) at the genus level. Only the presence and absence of bacteria defined to the phylum level in a) or to the genus level in b) were considered. Venn diagrams were generated with the package Venn diagram in R (what version). Most of the phyla were present before and after treatment.

The ants presented the lowest number of unique bacterial phyla and genera "before test", meaning before laboratory rearing conditions and any treatments. The highest number of unique phyla and genera was found after ampicillin treatment. 
Looking more specifically at the identity and the relative abundance of the 13 phyla, only two phyla were present $>1 \%$ in all treatments: Proteobacteria and Firmicutes (Table 3.1).

Table 3.1: Bacterial phyla in the Argentine ant before and after antibiotic treatments. The mean of the relative abundance of each phylum is presented in percentage, and the mean of raw number of reads identified for each phylum is in brackets. For phyla before the test and phyla after gentamicin treatment, no means were calculated as only one sample represented each treatment; the relative abundance and number of reads corresponded to the data from that single sample. Phyla present $>1 \%$ are considered non rare. Phyla non-rare in all treatment are in bold. Phyla present in all treatments are indicated by ${ }^{\wedge}$. OTUs that could not be classified as bacteria (category "Unclassified") and OTUs with non-named phyla are marginal, representing $<0.05 \%$ in total for before test and the control treatment. In order to compare bacteria presence between treatments, g-tests were realized on the relative abundance of the different phyla after rarefaction to 4,000 reads. Bonferroni corrected p-values are presented for each phylum. Significant p-values are indicated by a*. Only the control and ampicillin treatment were compared as they were the only treatments with two analysed samples.

\begin{tabular}{|c|c|c|c|c|c|}
\hline & \multicolumn{4}{|c|}{ treatment } & \multirow[b]{2}{*}{ Bonferroni corrected p-value } \\
\hline & Before.test & Control & Ampicillin & Gentamicin & \\
\hline Acidobacteria & $0.00(0)$ & $0.00(0)$ & $0.03(6)$ & $0.08(5)$ & NA \\
\hline Actinobacteria^^ & $0.22(9)$ & $0.53(37)$ & $\mathbf{3 . 4 7}(247.5)$ & $2.07(130)$ & $9.81 \mathrm{e}^{-76 *}$ \\
\hline Armatimonadetes & $0.00(0)$ & $0.005(0.5)$ & $0.00(0)$ & $0.02(1)$ & NA \\
\hline Bacteroidetes^$\wedge^{\wedge}$ & $0.07(3)$ & $0.20(16)$ & $0.34(55.5)$ & $0.41(26)$ & $2.15 \mathrm{e}^{-13 *}$ \\
\hline Chloroflexi & $0.00(0)$ & $0.02(1)$ & $0.004(1)$ & $0.00(0)$ & NA \\
\hline Cyanobacteria & $0.00(0)$ & $0.06(5)$ & $0.17(20.5)$ & $0.27(17)$ & NA \\
\hline Firmicutes $^{\wedge}$ & 32.25 (1336) & $14.66(1061)$ & $35.46(5779)$ & $10.29(646)$ & $0 *$ \\
\hline Fusobacteria & $0.00(0)$ & $0.00(0)$ & $0.004(1)$ & $0.00(0)$ & NA \\
\hline Planctomycetes & $0.00(0)$ & $0.00(0)$ & $0.02(2)$ & $0.00(0)$ & NA \\
\hline Proteobacteria $^{\wedge}$ & $67.19(2783)$ & $84.46(6735.5)$ & $\mathbf{6 0 . 0 5}(8167.5)$ & $86.78(5448)$ & $0^{*}$ \\
\hline Tenericutes & $0.24(10)$ & $0.00(0)$ & $0.01(3)$ & $0.02(1)$ & NA \\
\hline WPS-2 & $0.00(0)$ & $0.00(0)$ & $0.00(0)$ & $0.03(2)$ & NA \\
\hline WYO & $0.02(1)$ & $0.00(0)$ & $0.00(0)$ & $0.02(1)$ & NA \\
\hline
\end{tabular}

Actinobacteria was the only other non-rare phylum; Actinobacteria were non-rare after the ampicillin and gentamicin treatments. The relative percentages of Proteobacteria, Firmicutes, Actinobacteria and Bacteroides were significantly different between treatments. The control treatment bacterial diversity relative abundance differed from the "before test", indicating a change of bacterial diversity cause by laboratory rearing conditions. Furthermore, ampicillin and gentamicin treatments may have allowed an increase of Actinobacteria, Bacteroidetes and Cyanobacteria, and a decrease of Tenericutes compared to the before test and control treatment.

The difference in bacterial diversity between treatments may also be observed at the genera level (Figure 3.6 and Table 3.2). 


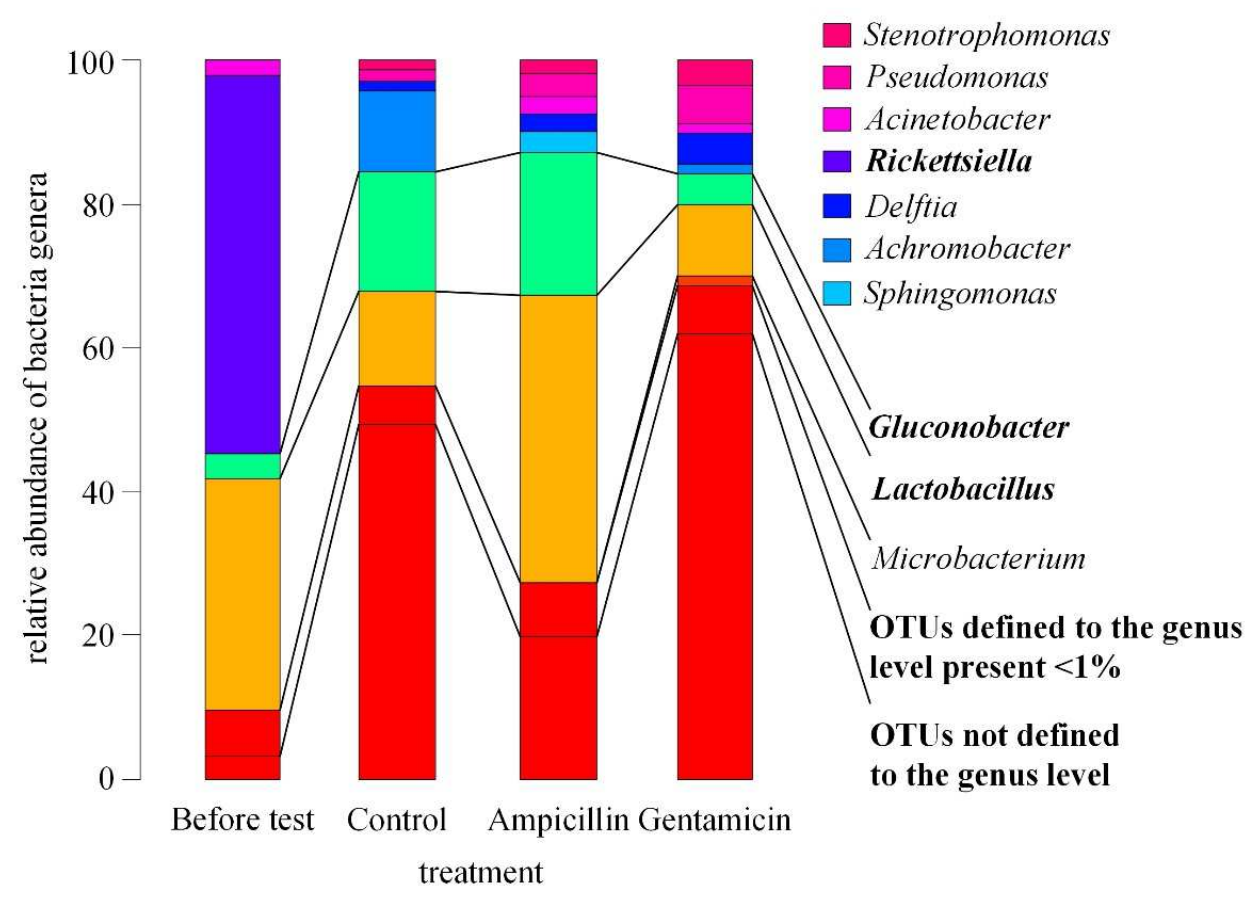

Figure 3.6: Relative abundance of non-rare bacteria genera before and after antibiotic treatments. Only non- rare bacteria genera, genera present $>1 \%$ in each treatment, are presented. Genera present $>10 \%$ in at least one treatment are in bold. The relative abundance of OTUs not defined to the genus level increases after laboratory rearing conditions, meaning the control treatment. 
Table 3.2: Bacteria genera present in Argentine ants before and after antibiotic

treatments. The relative abundance mean of each genus is presented in percentage and the mean of raw number of reads for each genus is in brackets. For "Before test" and "Gentamicin", no means were calculated as only one sample represented each treatment; the relative abundance and number of reads corresponded to the data from these single samples. Genera present $>1 \%$ are considered non rare and are in bold when nonrare in all treatments. Genera present in all treatments are indicated by ^. OTUs that could not be classified as bacteria (category "Unclassified") and OTUs with non-named phyla were representing $<0.3 \%$ in total in all treatments. In order to compare bacteria presence between countries, g-tests were realized on the relative abundance of the different genera after rarefaction to 4,000 reads. Bonferroni corrected p-values are presented for each genus. Significant p-values are indicated by a *. Only comparisons between the control and ampicillin treatments were realised as the two treatments were the only one composed of more than one sample.

\begin{tabular}{|c|c|c|c|c|c|c|}
\hline Phylum & Genus & Before test & Control & Ampicillin & Gentamicin & $\begin{array}{c}\text { Bonferroni } \\
\text { corrected } \\
\text { p-value }\end{array}$ \\
\hline Acidobacteria & Terriglobus & $0.00(0)$ & $(0)$ & $(0)$ & $0.05(3)$ & NA \\
\hline Actinobacteria & $\begin{array}{l}\text { Brevibacterium } \\
\text { Corynebacterium } \\
\text { Dermacoccus } \\
\text { Modestobacter } \\
\text { Cryocola } \\
\text { Leucobacter } \\
\text { Microbacterium } \\
\text { Mycobacterium } \\
\text { Nocardia } \\
\text { Rhodococcus } \\
\text { Aeromicrobium } \\
\text { Friedmanniella } \\
\text { Propionibacterium } \\
\text { Streptomyces } \\
\text { Tsukamurella } \\
\text { Rubrobacter }\end{array}$ & $\begin{array}{c}0.00(0) \\
(0) \\
(0) \\
(0) \\
(0) \\
(0) \\
(0) \\
(0) \\
(0) \\
(0) \\
(0) \\
(0) \\
0.05(2) \\
(0) \\
(0) \\
(0)\end{array}$ & $\begin{array}{c}(0) \\
0.04(2) \\
(0) \\
0.01(1) \\
0.005(0.5) \\
(0) \\
0.14(10) \\
0.14(7.5) \\
(0) \\
(0) \\
0.01(1) \\
(0) \\
0.08(5) \\
(0) \\
0.01(0.5) \\
0.04(4)\end{array}$ & $\begin{array}{c}0.05(11) \\
(0) \\
0.004(1) \\
0.01(2) \\
0.004(1) \\
0.01(3.5) \\
0.11(10) \\
2.74(140) \\
0.004(1) \\
0.012(7) \\
(0) \\
(0) \\
0.09(13) \\
0.01(1.5) \\
0.09(8) \\
(0)\end{array}$ & $\begin{array}{c}(0) \\
(0) \\
(0) \\
(0) \\
0.05(3) \\
(0) \\
1.43(90) \\
0.02(1) \\
(0) \\
(0) \\
(0) \\
0.03(2) \\
0.11(7) \\
(0) \\
0.30(19) \\
0.03(2)\end{array}$ & $\begin{array}{l}\text { NA } \\
\text { NA } \\
\text { NA } \\
\text { NA } \\
\text { NA } \\
\text { NA } \\
\text { NA } \\
\text { NA } \\
\text { NA } \\
\text { NA } \\
\text { NA } \\
\text { NA } \\
0.02 * \\
\text { NA } \\
\text { NA } \\
\text { NA }\end{array}$ \\
\hline Armatimonadetes & Fimbriimonas & $(0)$ & $0.005(0.5)$ & $(0)$ & $0.02(1)$ & NA \\
\hline Bacteroidetes & $\begin{array}{l}\text { Chryseobacterium }^{\wedge} \\
\text { Flavobacterium } \\
\text { Chitinophaga } \\
\text { Flavisolibacter } \\
\text { Segetibacter } \\
\text { Hymenobacter } \\
\text { Pontibacter } \\
\text { Spirosoma } \\
\text { Pedobacter } \\
\text { Sphingobacterium }\end{array}$ & $\begin{array}{c}0.02(1) \\
(0) \\
(0) \\
(0) \\
(0) \\
0.05(2) \\
(0) \\
(0) \\
(0) \\
(0)\end{array}$ & $\begin{array}{c}0.02(1.5) \\
(0) \\
(0) \\
(0) \\
0.005(0.5) \\
(0) \\
0.01(1.5) \\
(0) \\
0.04(2) \\
0.08(8)\end{array}$ & $\begin{array}{c}0.16(27) \\
0.01(2) \\
0.02(1) \\
(0) \\
0.09(16.5) \\
(0) \\
(0) \\
(0) \\
0.01(1.5) \\
0.002(0.5)\end{array}$ & $\begin{array}{c}0.18(8) \\
0.02(1) \\
(0) \\
0.03(2) \\
0.02(1) \\
(0) \\
(0) \\
0.03(2) \\
0.05(3) \\
0.02(1)\end{array}$ & $\begin{array}{c}1.80 \mathrm{e}^{-09} * \\
\text { NA } \\
\text { NA } \\
\text { NA } \\
\text { NA } \\
\text { NA } \\
\text { NA } \\
\text { NA } \\
\text { NA } \\
\text { NA }\end{array}$ \\
\hline Firmicutes & $\begin{array}{l}\text { Bacillus } \\
\text { Paenibacillus } \\
\text { Staphylococcus } \\
\text { Exiguobacterium } \\
\text { Enterococcus }\end{array}$ & $\begin{array}{l}(0) \\
(0) \\
(0) \\
(0) \\
(0)\end{array}$ & $\begin{array}{c}0.02(1.5) \\
0.02(1) \\
0.04(2.5) \\
(0) \\
(0)\end{array}$ & $\begin{array}{c}0.01(1.5) \\
(0) \\
0.07(11.5) \\
0.01(1.5) \\
0.01(2)\end{array}$ & $\begin{array}{c}(0) \\
(0) \\
0.02(1) \\
(0) \\
(0)\end{array}$ & $\begin{array}{l}\text { NA } \\
\text { NA } \\
\text { NA } \\
\text { NA } \\
\text { NA }\end{array}$ \\
\hline
\end{tabular}




\begin{tabular}{|c|c|c|c|c|c|c|}
\hline & $\begin{array}{l}\text { Lactobacillus }^{\wedge} \\
\text { Fusobacterium }\end{array}$ & $\begin{array}{c}32.23(1335) \\
0.00(0)\end{array}$ & $\begin{array}{c}14.34(1039.5) \\
0.00(0)\end{array}$ & $\begin{array}{c}35.13(5718.5) \\
0.004(1)\end{array}$ & $\begin{array}{c}10.02(629) \\
(0)\end{array}$ & $\begin{array}{l}0^{*} \\
\mathrm{NA}\end{array}$ \\
\hline Planctomycetes & Planctomyces & $0.00(0)$ & $0.00(0)$ & $0.02(2)$ & (0) & NA \\
\hline \multirow[t]{43}{*}{ Proteobacteria } & Brevundimonas & $0.00(0)$ & $0.00(0)$ & $0.004(1)$ & (0) & NA \\
\hline & Caulobacter $^{\wedge}$ & $0.05(2)$ & $0.12(8.5)$ & $0.38(38.5)$ & $0.25(16)$ & $5.85 \mathrm{e}^{-10 *}$ \\
\hline & Phenylobacterium & $0.00(0)$ & $0.01(1.5)$ & $0.02(1)$ & $0.00(0)$ & NA \\
\hline & Balneimonas & $0.00(0)$ & $0.005(0.5)$ & $0.01(2.5)$ & $0.03(2)$ & NA \\
\hline & Bosea $^{\wedge}$ & $0.07(3)$ & $0.42(29.5)$ & $0.49(89)$ & $0.67(42)$ & $1.11 \mathrm{e}^{-22 *}$ \\
\hline & Bradyrhizobium $^{\wedge}$ & $0.31(13)$ & $0.71(42)$ & $0.03(7)$ & $0.13(8)$ & $1.07 \mathrm{e}^{-08 *}$ \\
\hline & Ochrobactrum $^{\wedge}$ & $0.17(7)$ & $0.36(22.5)$ & $0.35(29)$ & $0.86(54)$ & $3.30 \mathrm{e}^{-09 *}$ \\
\hline & Devosia & $0.05(2)$ & $0.00(0)$ & $0.00(0)$ & $0.00(1)$ & NA \\
\hline & Methylobacterium ${ }^{\wedge}$ & $0.19(8)$ & $0.06(4.5)$ & $0.09(12)$ & $0.22(14)$ & 0.12 \\
\hline & Mesorhizobium & $0.00(0)$ & $0.00(0)$ & $0.00(0)$ & $0.03(2)$ & NA \\
\hline & Phyllobacterium & $0.00(0)$ & $0.00(0)$ & $0.70(35.5)$ & $0.00(0)$ & NA \\
\hline & Agrobacterium & $0.00(0)$ & $0.16(9.5)$ & $0.002(0.5)$ & $0.00(0)$ & NA \\
\hline & Kaistia & $0.00(0)$ & $0.03(3)$ & $0.004(1)$ & $0.00(0)$ & NA \\
\hline & Rhizobium & $0.02(1)$ & $0.00(0)$ & $0.02(1.5)$ & $0.00(0)$ & NA \\
\hline & Labrys & $0.14(6)$ & $0.00(0)$ & $0.00(0)$ & $0.00(0)$ & NA \\
\hline & Paracoccus & (0) & $0.005(0.5)$ & $0.01(1.5)$ & $0.00(0)$ & NA \\
\hline & Rhodobacter & $0.00(0)$ & $0.02(1)$ & $0.00(0)$ & $0.00(0)$ & NA \\
\hline & Rubellimicrobium & $0.00(0)$ & $0.02(2)$ & $0.01(2)$ & $0.13(8)$ & NA \\
\hline & Gluconobacter $^{\wedge}$ & 3.48 (144) & $22.62(1318.5)$ & $21.63(3507.5)$ & $4.22(265)$ & $0^{*}$ \\
\hline & Roseomonas & $0.14(6)$ & $0.01(1.5)$ & $0.00(0)$ & $0.02(1)$ & NA \\
\hline & Azospirillum & $0.00(0)$ & $0.00(0)$ & $0.03(6.5)$ & $0.00(0)$ & NA \\
\hline & Skermanella & $0.00(0)$ & $0.00(0)$ & $0.01(2.5)$ & $0.00(0)$ & NA \\
\hline & Rickettsia & $0.87(36)$ & $0.01(0.5)$ & $0.04(9.5)$ & $0.00(0)$ & NA \\
\hline & Wolbachia & $0.00(0)$ & $0.00(0)$ & $0.03(5)$ & $0.00(0)$ & NA \\
\hline & Sphingobium & $0.00(0)$ & $0.00(0)$ & $0.02(5.5)$ & $0.05(3)$ & NA \\
\hline & Sphingomonas $^{\wedge}$ & $0.92(38)$ & $1.56(69.5)$ & $\mathbf{3 . 1 9}$ (424) & $0.79(49)$ & $3.13 \mathrm{e}^{-129 *}$ \\
\hline & Sphingopyxis & $0.00(0)$ & $0.01(1)$ & $0.03(2)$ & $0.00(0)$ & NA \\
\hline & Achromobacter ${ }^{\wedge}$ & $0.10(4)$ & $8.75(881.5)$ & $0.62(56) 5$. & $\mathbf{1 . 4 3}(90)$ & $0^{*}$ \\
\hline & Burkholderia & $0.00(0)$ & $0.83(44.5)$ & $0.62(4)$ & $0.16(1)$ & NA \\
\hline & Acidovorax & $0.00(0)$ & $0.02(1)$ & $0.05(3.5)$ & $0.02(1)$ & NA \\
\hline & Delftia^ & $0.77(32)$ & $1.58(100)$ & $3.74(377)$ & $4.33(272)$ & $4.17 \mathrm{e}^{-92 *}$ \\
\hline & Diaphorobacter & $0.00(0)$ & $0.01(1)$ & $0.00(0)$ & $0.00(0)$ & NA \\
\hline & Methylibium & $0.00(0)$ & $0.00(0)$ & $0.01(1.5)$ & $0.00(0)$ & NA \\
\hline & Pelomonas & $0.00(0)$ & $0.09(6)$ & $0.28(30)$ & $0.25(16)$ & NA \\
\hline & Variovorax^ & $0.02(1)$ & $0.06(4)$ & $0.02(2)$ & $0.03(2)$ & 0.61 \\
\hline & Cupriavidus & $0.00(0)$ & $0.00(0)$ & $0.02(1.5)$ & $0.00(0)$ & NA \\
\hline & Janthinobacterium & $0.00(0)$ & $0.06(3)$ & $0.00(0)$ & $0.00(0)$ & NA \\
\hline & Ralstonia ${ }^{\wedge}$ & $0.02(1)$ & $0.047(3)$ & $0.26(20)$ & $0.06(4)$ & $4.17 \mathrm{e}^{-06 *}$ \\
\hline & Methylotenera & $0.00(0)$ & $0.00(0)$ & $0.004(1)$ & $0.00(0)$ & NA \\
\hline & Azospira & $0.00(0)$ & $0.00(0)$ & $0.00(0)$ & $0.05(3)$ & NA \\
\hline & Shewanella & $0.00(0)$ & $0.00(0)$ & $0.03(6.5)$ & $0.00(0)$ & NA \\
\hline & Citrobacter & $0.00(0)$ & $0.00(0)$ & $0.03(7)$ & $0.00(0)$ & NA \\
\hline & Enterobacter & $0.02(1)$ & $0.00(0)$ & $0.02(4)$ & $0.00(0)$ & NA \\
\hline
\end{tabular}




\begin{tabular}{|c|c|c|c|c|c|}
\hline Erwinia & 0.07 (3) & $0.01(1.5)$ & $0.12(6)$ & $0.00(0)$ & NA \\
\hline Escherichia $^{\wedge}$ & $0.05(2)$ & $0.13(8)$ & $0.27(34)$ & $0.49(31)$ & $1.65 \mathrm{e}^{-10 *}$ \\
\hline Gluconacetobacter & $0.00(0)$ & $0.00(0)$ & $0.55(27.5)$ & $0.05(3)$ & NA \\
\hline Serratia & $0.00(0)$ & $0.02(1.5)$ & $0.36(58.5)$ & $0.00(0)$ & NA \\
\hline Trabulsiella & $0.00(0)$ & $0.00(0)$ & $0.01(2)$ & $0.00(0)$ & NA \\
\hline Rickettsiella^ & $\mathbf{5 2 . 9 8}(2182)$ & $0.03(1.5)$ & $0.06(12.5)$ & $0.06(4)$ & $0 *$ \\
\hline Legionella & $0.00(0)$ & $0.04(2.5)$ & $0.00(0)$ & $0.00(0)$ & NA \\
\hline Acinetobacter ${ }^{\wedge}$ & $2.00(83)$ & $0.78(52.5)$ & $4.59(334.5)$ & $1.18(74)$ & $3.90 \mathrm{e}^{-71 *}$ \\
\hline Enhydrobacter & $0.00(0)$ & $0.005(0.5)$ & $0.00(0)$ & $0.03(2)$ & NA \\
\hline Psychrobacter & $0.00(0)$ & $0.00(0)$ & $0.01(3.5)$ & $0.00(0)$ & NA \\
\hline Pseudomonas $^{\wedge}$ & $0.63(26)$ & $2.01(126)$ & $\mathbf{3 . 5 0}(457)$ & $5.32(334)$ & $4.57 \mathrm{e}^{-122 *}$ \\
\hline Vibrio & $0.00(0)$ & $0.00(0)$ & $0.01(1.5)$ & $0.00(0)$ & NA \\
\hline Nevskia & $0.00(0)$ & $0.005(0.5)$ & $0.02(2.5)$ & $0.00(0)$ & NA \\
\hline Luteibacter & $0.00(0)$ & $0.00(0)$ & $0.01(3.5)$ & $0.00(0)$ & NA \\
\hline Pseudoxanthomonas & $0.00(0)$ & $0.04(2)$ & $0.09(5.5)$ & $0.05(3)$ & NA \\
\hline Stenotrophomonas $\wedge$ & $0.29(12)$ & $1.52(103.5)$ & $2.67(257)$ & $\mathbf{3 . 5 5}(223)$ & $4.48 \mathrm{e}^{-70 *}$ \\
\hline
\end{tabular}

While the reads from the same treatment samples were pooled together to create the bar charts, the table (Table 3.2) corresponds to the mean relative abundance and number of reads per treatment. Consequently, the two representations of the results may present a few differences. Based on the bar chart (Figure 3.6), the sum of the relative abundance of rare genera before and after the treatment was similar (mean $=6.44 \pm 0.47 \%$ ). The relative abundance of OTUs not defined to the genus level highly increased after laboratory rearing conditions and antibiotic treatments (Figure 3.6 and Table 3.3). "Before test", meaning after field collection and before laboratory rearing conditions, presented only $3.26 \%$ of OTUs non-defined to the genus level. After the control and antibiotic treatments, the proportion of OTUs non-defined to the genus level increased to $19.91 \%$ for the ampicillin treatment, $49.43 \%$ for the control, and $61.82 \%$ for gentamicin. These non-defined OTUs may induce a bias in the relative abundance of the bacteria defined to the genus level. Even with a high abundance of non-defined OTUs, the number of bacterial genera present after the treatments was higher than before laboratory rearing conditions. Four bacteria genera were non- rare in the before test sample: Rickettsiella, Lactobacillus, Gluconobacter and Acinetobacter, in decreasing order of relative abundance. After treatments, six genera were non-rare in the control (from most to less abundant: Gluconobacter, Lactobacillus, Achromobacter, Pseudomonas, Stenotrophomonas and Delfia,), seven in the ampicillin (Lactobacillus, Gluconobacter, Pseudomonas, Sphingomonas, Delfia, Acinetobacter, and Stenotrophomonas) and eight in the gentamicin treatment (Lactobacillus, Pseudomonas, Gluconobacter, Delfia, Stenotrophomonas, Achromobacter, Microbacterium and 
Acinetobacter). In the Table 3.3, Mycobacterium was also non-rare after ampicillin treatment. Ampicillin and gentamicin treatments increased the relative abundance of Acinetobacter. In addition, ampicillin treatment increased the relative abundance of Lactobacillus and Sphingomonas, and decreased Achromobacter. For its part, gentamicin treatment increased Delfia and decreased Gluconobacter. In total, 22 bacteria were present before and after treatment in all samples (Table 3.2).

\subsubsection{Ant survival and colony fitness after antibiotic treatments}

The long term survival of colonies treated with antibiotic varies between antibiotic treatments (Figure 3.7).

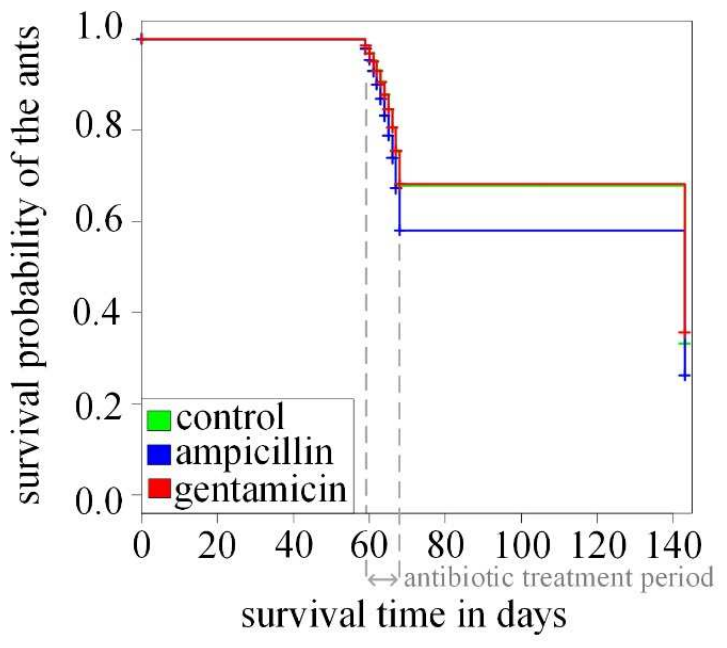

Figure 3.7: Survival probability of Argentine ants during and after antibiotic

treatments. Each treatment (control, ampicillin and gentamicin) survival results correspond to the mean survival of workers from three colonies (day $0, n=300$ workers +3 queens +15 larvae).

There were significant differences between the ampicillin treatment and the control (coxph, coef $=0.2980, \operatorname{se}($ coef $)=0.0290, \exp ($ coef $)=1.347, \mathrm{z}=10.263, \mathrm{p}<0.001)$, and between the ampicillin and gentamicin treatments (coxph, coef $=-0.319$, se(coef) $=0.0292$, $\exp ($ coef $)=0.727, \mathrm{z}=-10.9, \mathrm{p}<0.001)$. Ampicillin treated colonies presented significantly lower survival probability than the control and the gentamicin treated colonies. There were 
no significant differences between the gentamicin treatment and the control (coxph, coef $=$ $0.0208, \operatorname{se}($ coef $)=0.0311, \exp ($ coef $)=0.979, z=-0.667, p>0.05)$.

The brood, meaning the eggs and the larvae, were considered to be an indicator of the fitness of the colony in a generalised linear model (GLM). The treatments, the number of bacteria, and the number of workers and of queens did not significantly affect the colony fitness (Table 3.3).

Table 3.3: Results of the Generalised linear model (GLM) on colony fitness (number of brood) and the significance of the antibiotic treatments, the number of bacteria, workers and queens as factors. No differences were statistically significant.

\begin{tabular}{l|cccc} 
& Estimate & Std. Error & $\mathrm{t}$ value & $\operatorname{Pr}(>|\mathrm{t}|)$ \\
\hline Ampicillin treatment & -3.96619 & 2.54394 & -1.559 & 0.1449 \\
Gentamicin treatment & -5.18199 & 2.71382 & -1.909 & 0.0804 \\
Number of bacteria & -0.15711 & 0.09521 & -1.65 & 0.1248 \\
Number of workers & -0.09625 & 0.065 & -1.481 & 0.1644 \\
Number of queens & 3.16222 & 2.09136 & 1.512 & 0.1564
\end{tabular}

From the multiple factors, gentamicin ( $\mathrm{p}$ close to 0.05 ) seemed to be the only factor affecting the colony fitness.

\subsection{Discussion}

Among the 22 bacteria present before and after treatments in Argentine ants, Lactobacillus and Gluconobacter were the only bacteria non-rare in all samples. Lactobacillus and Gluconobacter can be found in insects, among others (Crotti et al., 2010; Martinson et al., 2011; Coon et al., 2014; Corby-Harris et al., 2014; Maistrenko et al., 2015). Lactobacillus is a favourable bacterial genus that includes bacteria known for their multiple beneficial proprieties: growth promotion in flies (Drosophila sp) (Storelli et al., 2011), and protection against bacterial and fungal infections in bees (Apis spp.) (Vásquez et al., 2012) and ants (Mycocepurus smithii) (Kellner et al., 2015). The bacteria from this genus can be acquired horizontally in the host environment (Kellner et al., 2015) or by trophallaxis between nestmates (Vásquez et al., 2012), and vertically from parents to offspring (Storelli et al., 2011). For its part, Gluconobacter has been reported to include bacteria species with both beneficial and harmful proprieties. In honey bees (Apis spp.), bacteria from the 
Gluconobacter genus are part of their host core bacteria and are involved in their host health (Anderson et al., 2011; Martinson et al., 2011). Alternatively, some Gluconobacter bacteria are pathogenic in flies (Drosophila spp.), and have been reported to induce chronicle gut inflammation in their hosts (Matos and Leulier, 2014). To our knowledge, Gluconobacter have not been previously reported in any ant species. The presence of Gluconobacter bacteria in all Argentine ants may indicate a beneficial relationship between the ants and bacteria from this genus as with bees unlike the detrimental relationship with flies. Lactobacillus and Gluconobacter bacteria may participate to the Argentine ant immune system, preventing pathogenic infections in individuals and in the colonies. Finding antibiotics active against bacteria from these genera may offer knowledge on their relationship with Argentine ants.

\section{Antibiotic treatments increase bacterial diversity}

Bacterial diversity increased not only after treatment with ampicillin and gentamicin but also increased in the control compared to the bacterial diversity of Argentine ants in the field. Firstly, laboratory rearing conditions surprisingly increased bacterial diversity present in Argentine ants. In the field, Argentine ants feed on other arthropods and honeydew from mealybugs. In the laboratory, Argentine ants are presented to different environmental conditions. Bacteria sources for the ants are limited mostly to the food source, honey and mealworm larvae (Tenebrio molitor) and somewhat be considered simple (Wang and Zhang, 2015). The only non-rare bacterial genus present in both mealworms and Argentine ants is Lactobacillus (Wang and Zhang, 2015; Stoops et al., 2016). The increase of bacterial diversity in reared Argentine ants cannot be solely attributed to the exclusive feeding on mealworms. Feeding on honey may improve the Argentine ant health through antimicrobial and antifungal proprieties (AL-Waili et al., 2013; Gherman et al., 2014). Honey proprieties may also affect the ant bacteria and their relative abundance. Some opportunistic bacteria may grow their population, modifying further the change in the observed bacterial diversity. These opportunistic bacteria may not have been detected in ants in the field because of reduced abundance. The association between honey and the mealworms as food source for the ants in the laboratory may explain the change in bacteria relative abundance, as the decrease of Rickettsiella in high relative abundance only in ants collected in the field, and the increase of bacterial diversity in the control ants. 
Ampicillin and gentamicin further increased the observed change in bacterial diversity observed in the ants. The two antibiotics affect bacterial genera differently. Ampicillin kills both Gram positive and Gram negative bacteria while gentamicin kills Gram negative bacteria and selected Gram positive bacteria. As a consequence, the two antibiotic treatments modified the bacteria equilibrium differently in the ants. Opportunistic bacteria may increase their populations while the populations of bacteria affected by the antibiotics decrease. Ampicillin and gentamicin treatments indirectly promoted the growth of the populations of some bacteria such as Acinetobacter, as well as Lactobacillus and Sphingomonas for ampicillin and Delfia for gentamicin. Ampicillin decreased Achromobacter population, and gentamicin decreased the population of Gluconobacter, the two observed decreases of non-rare bacteria genus populations after antibiotic treatments.

It is noteworthy to highlight the high abundance of Rickettsiella genus in the field collected ants, and the absence of the genus after the colony acclimation. Rickettsiella is a genus of pathogenic bacteria infecting arthropods (Iasur-Kruh et al., 2013). The rearing of the ants in the laboratory and the change in environmental conditions may have allowed the disappearance of the infections in the ants.

\section{Antibiotic treatments decrease ant survival but not fitness}

Ampicillin treated ants presented a lower survival probability than non-treated ants while gentamicin treated ants did not seem to have their survival probability affected. These results may be due to the differences in bacterial diversity between ampicillin and gentamicin treated ants. Sphingomonas was present $>1 \%$ in ampicillin treated ants but not in the gentamicin treatments. Sphingomonas is a genus of bacteria found in plants and insects among others (Gayatri Priya et al., 2012; Tang et al., 2012; Carrasco et al., 2014) such as ants (Ishak et al., 2011b). Bacteria from this genus have been reported to protect plants and bees (Apis spp.) against pathogenic infections (Innerebner et al., 2011; Kaltenpoth and Engl, 2014). While Sphingomonas seems to be a genus of beneficial associates, the effects of the bacteria of this genus in ants are unknown. It is possible that

the increase in the genus relative abundance in the ampicillin treated Argentine ants may have been the cause of the decrease of the ant survival. Achromobacter is the only bacterial genus present $>1 \%$ in both the control and the gentamicin treatments but not in the ampicillin treatment. Achromobacter includes bacteria reported in multiple organisms 
including plants and insects like ants (Ishak et al., 2011b; Hernández et al., 2013; Jha and Saraf, 2015). Some bacteria from the genus are involved in their host plant nutrition and growth (Jha and Saraf, 2015).The effects of bacteria of this genus on their insects host are unknown. The increase of bacterial diversity combined to the change in relative abundance of the different bacteria genera caused by ampicillin treatments may therefore be at the origin of the decrease in the ant survival.

Neither ampicillin nor gentamicin treatments significantly modified Argentine ant fitness or brood production. Brood production may not have been affected by the antibiotic treatments if the queens did not receive the treatments. Argentine ant foragers ingested large quantities of the antibiotic-honey mix in order to feed the entire colony. Once in the colony, foragers shared the food with nurses by trophallaxis, and in turn, nurses feed the queens and larvae by trophallaxis. There was a disparity in the quantity of antibiotics

foragers, nurses, larvae and queens were exposed to. Foragers were exposed to a higher concentration of antibiotics compare to queens and larvae. As a consequence, the queen bacterial diversity may not have been affect by the antibiotic treatments as much as foragers had, and the treatments may not have at length affected the brood production. This disparity in antibiotic exposure may explain the observed decrease of ant survival while the colony fitness was not affected by the treatments. Additional bacterial diversity data based on the bacteria present in the queens in the field, after laboratory rearing conditions and after antibiotic treatments may offer additional information.

\section{Critical evaluation of experimental procedures}

Next generation sequencing allows the sequencing of large amounts of data. However, costs are high and limit the amount of possible analyse data. If 454 sequencing did not limit the number of analysed samples, it would have been interesting to test the four antibiotics (ampicillin, gentamicin, kanamycin and spectinomycin) effects on bacterial diversity and Argentine ant fitness. Idealy, each treatment would be tested on three colonies, 15 colonies in total including the control treatment ones. If possible, the bacterial diversity may be analysed to follow the change during laboratory rearing and antibiotic treatments by collecting samples: 1) in the field; 2) acclimation and before treatment; 3) after the 10 day antibiotic treatments; 4) at the end of the experiment, and 5) after 15 days without treatments. The selected antibiotics are bactericides, meaning that they kill bacteria. Dead 
bacteria may still have been present in the ants when DNA is extracted from the ants for 454 sequencing. Allowing a few days (or 15 days) for the ants to eliminate the dead bacteria from their organelles may allow a better characterisation of the change in bacterial diversity following the antibiotic treatments. This degradation of dead bacteria and their genetic material may explain the increase of non-assigned OTUs after antibiotic treatments. The bacterial DNA amplified may be fragmented because the bacteria were dead and being eliminated by the ant organisms. Analysing at least two samples from each treatment would statistically better support the findings. Using three samples from the field as well as three from each treatment (one sample per colony replicate) during time would make the data analyses more robust. Furthermore, adding samplings along the experiment time line would allow a better characterisation of the bacterial diversity present in the ants.

Antibiotic treatments on Argentine ant colonies associated with 454 sequencing offered the opportunity to test the effects of antibiotics on bacterial diversity present in the ants, and observed the consequence of the change in bacterial diversity on Argentine ant survival and fitness. Ampicillin and gentamicin treatments increased bacterial diversity present in the ants. Only ampicillin treatments decreased Argentine ant survival probability. This decrease in survival probability may be due to the change in bacterial diversity and relative abundance. Neither antibiotic affected colony fitness as the queens may not have been exposed to enough antibiotic to affect their bacterial diversity. Further antibiotic treatments targeting directly queens may offer a better understanding of the change in bacterial diversity on colony fitness. This study offers a first insight regarding the effects of environmental modifications on bacterial diversity and their consequences on Argentine ant population. The change in bacterial diversity may also alter Argentine ant recognition behaviour and increase the ant aggression behaviour. The ant population may thrive with low intra-specific aggression levels to promote Argentine ant cooperation and high intraspecific aggression levels to increase the ant competitively. Antibiotic treatments may artificially modify the ant bacterial diversity and allow me to test the effects of a change in bacterial diversity on ant inter- and intra-specific aggression levels and encounter survival (see Chapter 4). 
Chapter 4. Argentine ant inter-species aggressiveness increases in the absence of fitness changes after antibiotic treatments 


\subsection{Abstract}

Populations fluctuate, going through possible multiple decline events. Bacteria may be involved in decreasing or promoting their host populations. A change in bacterial diversity may decrease their host health, their aggressiveness and their chance of surviving interspecies encounters. These changes may lead at length to possible population declines. The invasive Argentine ant is a successful pest that has spread worldwide from South America, at the expense of native species. In New Zealand, the success of these invasive ants is in part due to their high density population and their aggressive dominance behaviour. The lack of aggression between workers from different nests allows cooperation between nests and high number of workers defending food sources against other species. Antibiotics may be used to modify bacterial diversity present in Argentine ant, and assess the effects of the change of bacterial diversity on the ant colony survival, fitness and aggressiveness. Ampicillin, kanamycin and spectinomycin did increase Argentine ant colony survival. However, the antibiotics did not affect the ant fitness nor intra-species aggression. Spectinomycin did increase the Argentine ant aggressiveness during intra-species encounters with New Zealand native Prolasius advenus ants. These results indicate that a change in bacterial diversity may increase the ant health and survival. An increase of interspecies aggressiveness may also increase Argentine ant competitiveness against native species. The change in bacterial diversity present in invasive ants may be involved in invasive species population dynamics and aggression behaviour. 


\subsection{Introduction}

\section{Pathogenic and favourable bacteria associated with population dynamics}

Population of invasive species show considerable variation, with possible population declines following booms. The invasive yellow crazy ant (Anoplolepis gracilipes) populations in Tokelau, Christmas Islands and Australia displayed extreme population reduction after extensive population increase (Abbott, 2006; Gruber et al., 2012; Cooling and Hoffmann, 2015). However, the cause of these population expansions and declines are not yet known for these ants. In the case of the worldwide introduced honey bee (Apis mellifera), extreme population declines were reported in multiple countries from different continents. This phenomena, named colony collapse disorder (CCD), was associated to multiple factors including pathogenic bacterial and viral infections (Evans and Schwarz, 2011). Pathogenic infections correspond to infections by micro-organisms, such as bacteria and viruses, causing illness in their hosts. The American foulbrood agent (Paenibacillus larvae) kills infected bee larvae (Apis spp.) and can lead at length to the death of entire bee colonies (Genersch, 2010a). Pathogenic infections were also hypothesized as possible cause for invasive ant population decline (Cooling et al., 2012; Cooling and Hoffmann, 2015). Already three viruses causing colony decline and possible death of entire nests have been described in the invasive red imported fire ant (Solenopsis invicta): Solenopsis invicta virus -1, -2 and 3 (Oi and Valles, 2009; Valles et al., 2013a; Manfredini et al., 2016).

Favourable bacteria may at the contrary promote their host health and indirectly their fitness and population growth (Boursaux-Eude and Gross, 2000; Zientz et al., 2005; Russell et al., 2009). Favourable bacteria can help their hosts against pathogenic infections (Koch and Schmid-Hempel, 2011; Mattoso et al., 2012). For example, some Lactobacillus bacteria found in the gut of bees (Apis spp.) are involved in their host health and immune system, protecting their host against some bacterial and viral infections (Yoshiyama and Kimura, 2009). Other bacteria are necessary to their hosts for nutrition and host development. Buchnera bacteria are associated with aphids (Douglas, 1998). Aphids without the bacteria do not develop as well as infected aphids, and produce fewer or no offspring at all. The loss of favourable bacteria may consequently decrease their host's fitness. If the loss is widespread, the absence of favourable bacteria may induce a decline of their host population. 


\section{Bacteria involvement in aggressive behaviour in social insects}

In the case of social insects, bacteria present in individuals may influence individual aggressiveness against nestmates but also against other species. Nestmate recognition is influences by multiple factors in addition to the colony cuticular hydrocarbon $(\mathrm{CH})$ signature (Jones et al., 2012; Turgis and Ordon, 2012). CH are molecules found on the insects, and are frequently specific to colonies. Colony $\mathrm{CH}$ signature may be modified temporarily by contact with the environment, and influenced by the individual diet (Liang et al., 2001; Corin et al., 2007a). Furthermore, recognition between nestmates may also be influenced by the bacterial diversity present in the individuals as it is the case for some termites (Reticulitermes speratus) (Matsuura, 2001). A major change in bacterial diversity of some termites in a nest can lead to an increase of aggression within that nest. Nestmates with different bacterial diversity would not be recognise as nestmates anymore. In the case of supercolonies, the changes in $\mathrm{CH}$ signatures may lead to the non-recognition of nests between each other, and at length to the fragmentation of the supercolony. Supercolonies are colonies compose of a high number of nests spread on a large space, and presenting a lack of aggression between workers from different nests (Heller et al., 2008b). The appearance of intra-species aggression in a supercolony may potentially weaken the supercolony and lead to its decline.

Population dynamic is also associated with individual aggressiveness level against other species to defend food sources, territory and nests. Invasive social insects present high level of inter-species aggressions (Fadmiro et al., 2009; Hudina et al., 2014). A decrease in aggressiveness of social insects may lead to reduce food access and at length limit colony growth. A change in bacterial diversity may decrease individual health and lower hots aggressiveness and resistance during inter-specific encounters. As bacterial diversity has been associated with individual health, it may be possible that bacterial diversity may affect individual aggressiveness behaviour and survival. On a colony level, the change of bacterial diversity present in the queens, workers and brood may affect their intra- and inter-species aggressiveness.

\section{Antibiotic treatments}

As discussed in Chapter 3 (section 3.2), antibiotics may be used in order to modify bacterial diversity present in individuals. Antibiotics offer an insight on the effects of the loss of 
bacteria and change in bacterial diversity on treated individual fitness and survival. For example, antibiotic treatments affecting the family of bacteria Rickettsiaceae that includes Wolbachia decrease development and fitness of Brugia malayi worms in vitro (Rao and Weil, 2002). Antibiotic treated leaf-cutter ants (Acromyrmex subterraneus subterraneus) were more susceptible to fungal infections and therefore experienced reduced survival (Mattoso et al., 2012).

For social insects, the antibiotic treatments may be offered to all colony members by mixing the antibiotics with food. Foragers retrieve food for their colony. Via trophallaxis, or to mouth exchange, foragers pass along food to nestmates including nurses. Nurses will then feed the queens and brood. The effects of antibiotics may therefore observed on the entire colony fitness and survival.

\section{The case of the invasive Argentine ant}

Native from South America, Argentine ants (Linepithema humile) are an invasive ant species in New Zealand. While Argentine ants are a successful invasive species, spreading at the expense of native ants species and other competitors, the invasive ant population inexplicably declined in New Zealand (Cooling et al., 2012). Pathogenic infections have been proposed as the cause of this population decline. Pathogenic infections may have weakened infected individuals and their colonies, lowering their aggressive competitiveness and food resources. Pathogenic infections may also have modified the interactive behaviour between nests. Uninfected nests may have displayed aggressive reactions toward infected ants. Intra-species aggression may have resulted in a fragmentation of the supercolony and lower Argentine ant numbers. At length, Argentine ant fitness may have declined and resulted in the population decrease. Antibiotics may be used on laboratory Argentine ant colonies to change their bacterial diversity (see Chapter 3 ) and to induce a stress in order to affect the ant survival, fitness and aggressiveness. The results may inform on the role of bacterial diversity on Argentine ant population dynamics.

\section{Chapter aims}

This chapter aims to characterise the effects of antibiotic treatments, firstly on Argentine ant intra- and inter-species aggressiveness, and secondly on Argentine ant colony fitness and survival. Antibiotic treatments were hypothesised to change the bacterial diversity 
present in ants which in turn could: 1) increase intra-species aggressiveness; 2) increase inter-species aggressiveness; and 3) increase Argentine ant colony fitness and survival.

\subsection{Materials and methods}

\subsubsection{Ant colony collection and laboratory conditions}

Multiple nests of Argentine ants were collected in a private vineyard in Hastings, New Zealand (lat. -39.60; long. 176.74), in April 2014. Ant nests were pooled together as Argentine ants are unicolonial in New Zealand, and ants from different nests do not act aggressively towards each other (Corin et al., 2007a). In the laboratory, 20 sub-nests (now on considered as colonies) were created. Each colony was composed of five queens, 700 workers, and brood. The colonies were placed in plastic boxes $(20.5 \times 14.0 \times 7.0 \mathrm{~cm})$ that had walls coated with Fluon ${ }^{\mathrm{TM}}$ (Polytetrafluoroethylene PTFE-30; BioQuip Products, Inc.). Each box contained three glass tubes $(1.5 \mathrm{~cm} \varnothing ; 15.0 \mathrm{~cm}$ long) filed to one third with water (the water was blocked by a cotton ball) and covered in tin foil to create a favourable nesting environment. For intra-specific interactions described below, one additional large colony of Argentine ants was created with one queen, $\sim 5,000$ workers and brood. This colony was placed in a large plastic box $(25.5 \times 25.5 \times 13.0 \mathrm{~cm})$ with walls coated with Fluon ${ }^{\mathrm{TM}}$ and containing 10 glass tubes prepared as previously described for nesting. The colonies were maintained at $20^{\circ} \mathrm{C} \pm 1$, under a $16 / 8 \mathrm{~h}$ light-dark cycle. They were fed twice a week with a cotton ball soaked in $\sim 5 \mathrm{~mL}$ of a $20 \%$ honey solution $(\mathrm{v} / \mathrm{v})$ and $\sim 1.5 \mathrm{~g}$ of fresh cut mealworms (Tenebio molitor larvae). After two months of colony acclimation and prior to the experiments described below, the number of workers and queens were counted again, and all the brood was removed.

For inter-specific interactions, New Zealand native ants Prolasius advenus were selected. Six colonies of $P$. advenus were collected in Kaitoke Regional Park in New Zealand (Rivendell - lat. -41.06; long. 175.18) in May 2014. They were placed in nests and kept in conditions similar to the Argentine ants, as described previously. 


\subsubsection{Antibiotic treatments}

Argentine ant colonies were treated with antibiotics in order to modify the bacterial diversity present in the ants (see Chapter 3). Ampicillin, kanamycin and spectinomycin were selected for their bactericidal proprieties. More specifically, ampicillin is a $\beta$-lactam antibiotic interfering with the construction and the functionality of the bacteria cell wall and is a broad spectrum of Gram positive and Gram negative bacteria. Kanamycin and spectinomycin are two aminoglycoside antibiotics inhibiting protein synthesis in bacteria Gram negative and some Gram positive. Rifampicin was also considered as a potential treatment, as rifampicin is a bactericidal antibiotic with a different interference compared to the other selected antibiotics. Rifampicin inhibits the synthesis of RNA in bacteria. However, ants would not feed on solutions containing this antibiotic (see Appendix III). Rifampicin was consequently disregarded as a potential antibiotic treatment.

Argentine ant colonies underwent four treatments $(n=5$ colonies for each treatment): no antibiotics (control), ampicillin, kanamycin, and spectinomycin. The first part of the experiment corresponded to a short-term antibiotic treatment. Colonies were fed every day for five days a $200 \mu \mathrm{L}$ mix composed of $20 \%$ (w/v) sucrose and $5.00 \mathrm{mg} / \mathrm{mL}$ antibiotics (final concentrations). The control colonies were feed with a $20 \%(\mathrm{w} / \mathrm{v})$ sucrose solution. The mix was offered to the ants on a $2 \mathrm{~cm}^{2}$ tin foil cut. Starting $10 \mathrm{~min}$ after the placement of food in the foraging area of the boxes, the number of ants feeding on the mix were recorded every $10 \mathrm{~min}$ for $100 \mathrm{~min}$ to ensure feeding. At the end of the five day antibiotic treatments, the colonies were fed $\sim 1.5 \mathrm{~g}$ of freshly killed mealworms. The second part of the experiment or long-term antibiotic treatment then followed. Colonies were fed three times a week for three weeks an antibiotic mix (the same as the one during the first period). After each antibiotic feeding, the number of ants feeding was counted as described above. In addition, after the treatment feedings, the colonies were given $\sim 1.5 \mathrm{~g}$ of freshly killed mealworms twice a week.

\subsubsection{Changes in inter- and intra-specific aggressive interactions}

The modification of intra and inter-species aggressiveness and survival rate of Argentine ants after the antibiotic treatments was investigated. Argentine ant $(\mathrm{n}=10)$ aggressiveness and survival were recorded during encounters with untreated Argentine $(\mathrm{n}=10)$ ants and $P$. advenus $(\mathrm{n}=10)$. Untreated Argentine ants were marked with one blue dot on the gaster to differentiate them from treated Argentine ant workers. Ants were randomly selected from 
the colonies for each replicate and discarded after the experiment to avoid selecting the ants again in the subsequent study and maintain the presence of only naïve ants in the encounters. The interaction arenas consisted in plastic boxes $(13.5 \times 9.0 \times 8.0 \mathrm{~cm})$ with walls coated in Fluon ${ }^{\mathrm{TM}}$. In each box, a plastic ring $(5.0 \mathrm{~cm} \varnothing ; 5.0 \mathrm{~cm}$ high) with both sides coated in Fluon ${ }^{\mathrm{TM}}$ was placed in the middle. Each ant group was randomly assigned to a side (outside or inside) of the ring. After placement, the ants were left to acclimatise for 20 min. The ring was then removed. During each fight, aggressive behaviour was scored following Barbieri (Thesis, 2014): score 0 - "ignore" (body contact with no reaction); score 1 - "touch" (one ant taps the other with its antennae); score 2 - "avoid" (after contact, one of the individual runs away from the other ant in the opposite direction); score 3 "aggression" (one ant bite a body part of the other ant or raised its gaster); score 4 "fighting" (prolonged aggression of at least $5 \mathrm{~s}$ ). Behaviour interactions were scored during $25 \mathrm{sec}$ every $2 \mathrm{~min} 30 \mathrm{sec}$ for a total period of $25 \mathrm{~min}$. To assess survival, the number of dead ants was recorded at $\mathrm{t}=25 \mathrm{~min}, 30 \mathrm{~min}, 40 \mathrm{~min}, 50 \mathrm{~min}, 1 \mathrm{hr}, 2 \mathrm{hr}, 4 \mathrm{hr}, 8 \mathrm{hr}$ and $16 \mathrm{hr}$. Interaction encounters were conducted before (day 0), after five days (day 5) and after a month (day 30) of antibiotic treatments. Each colony participated to two encounters with untreated marked Argentine ants and two encounters with $P$. advenus. For each treatments, 10 pseudo-encounter replicates were realised in total for intra-encounters and 10 pseudo-encounter replicates for inter-encounters.

Prior to conducting interaction tests, the absence of effects of marking and painting Argentine ants on aggressiveness and survival was assessed. Following the experimental protocol previously described, the aggressiveness score and survival rates were recorded for encounters $(n=10$ replicates) between marked untreated Argentine ants $(n=10)$ and unmarked Argentine ant $(\mathrm{n}=10)$, and encounters $(\mathrm{n}=10$ replicates $)$ between marked untreated Argentine ants $(n=10)$ and untreated Argentine ants marked differently $(n=10)$. In addition, control of survival probability in the absence of opponents were realised for pre-treated Argentine ants, marked untreated Argentine ants and P. advenus, following the protocol previously described, on day 0 before any treatments. For each treatment group of Argentine ants, 10 survival replicates were realised, two pseudo-replicate encounters per colony. For marked Argentine ants, 10 pseudo-replicates encounters were realised using workers from the large Argentine ant colony set aside. Six replicates were done for $P$. advenus encounters, one per colony. Control of the survival probability of treated Argentine 
ant workers in absence of aggressive encounters were realised also after five days (day 5) and after a month (day 30) of the beginning of the antibiotic treatments.

\subsubsection{Survey of survival and fitness of colonies}

To assess the effects of antibiotic treatments on ant survival in the colony, the number of ants were counted before the start of the antibiotic treatments and at the end of the experiment. The number of eggs and larvae were counted to assess the fitness of the colony (based on brood production) after the antibiotic treatment at the end of the experiment.

\subsubsection{Data analysis}

All analyses and figures were realised in $\mathrm{R}$ version i386 3.1.3. Differences in aggressiveness scores of treated Argentine ants were analysed using a generalised linear model (GLM) with treatment, time and opponent scores (the scores of either untreated Argentine ants or P. advenus encountering the treated ants) as variables. The survival probability of ants in different treatments after encounters was analysed using the package survival as described in Chapter 4 (section 3.3.4). Comparison between survival probabilities of ants in different treatments was analysed using Cox proportional hazard regression models (Barbieri et al., 2013). The survival of ants in the treated colonies and the fitness of the colonies (brood production, i.e. eggs and larva) were compared using generalised mixed models (GLM) based on treatments, the number of queens and time. Significance for all tests was assumed at $\mathrm{p}<0.05$.

\subsection{Results}

\subsubsection{Tests prior to antibiotic treatments}

\section{Argentine ant survival in the absence of aggressive encounters}

The survival of ants prior to any treatments (day 0) and in absence of any aggressive encounters was analysed (Figure 4.1). 


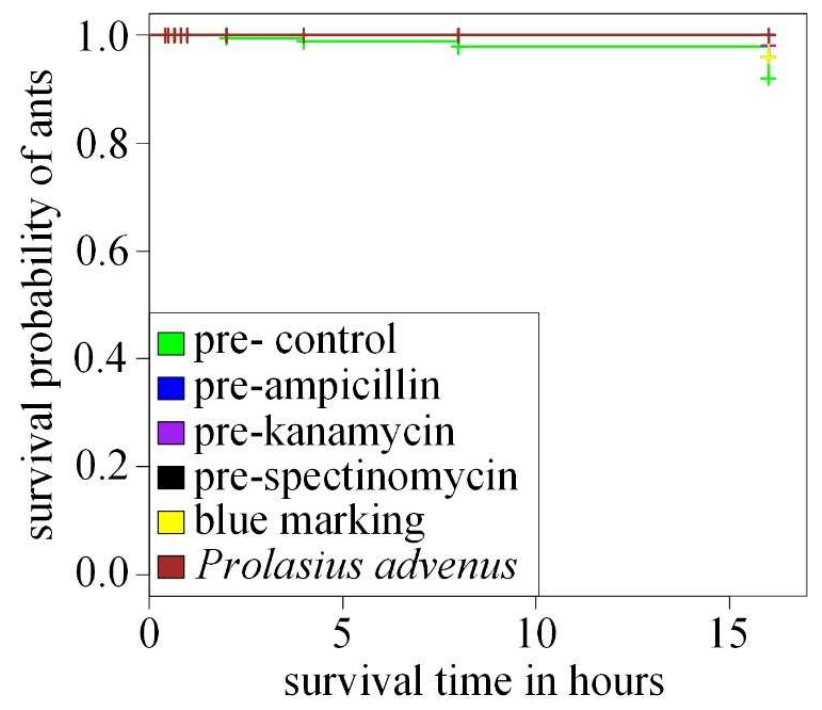

Figure 4.1: Survival probability of ants in the absence of aggressive encounter and prior to any antibiotic treatments. Each survival probability line is the mean survival of all the replicates of groups of workers $(\mathrm{n}=10)$ for each pre-treatment, for blue marking and for Prolasius advenus colonies. Each group of workers came from one colony: unmarked Argentine ants, from each of the five colonies for each future treatment (control, ampicillin, kanamycin and gentamycin), five pseudo-replicates of Argentine ants with blue marking from the large colony set aside, and Prolasius advenus from each of the six colonies.

Survival probability of Argentine ants in the absence of aggressive encounter prior to any antibiotic treatments was close to $100 \%$ for 16 hours on day 0. Similarly, the survival of Argentine ants marked in blue and the survival of $P$. advenus in the absence of aggressive encounters was close to $100 \%$ for 16 hours. There was significant differences in the survival between groups of ants in the absence of aggressive encounter (Likelihood ratio test $=14.6$, $\mathrm{df}=5, \mathrm{p}<0.05, \mathrm{n}=2790 ; \mathrm{coxph})$. However, no significant differences were found between any pair of ant groups (Table 4.1).

Table 4.1: P-values results of the Cox survival analysis in the absence of aggressive encounters between each of the ant groups. Survival was analysed for Argentine ant survival prior to treatments, Argentine ants marked with blue and Prolasius advenus.

\begin{tabular}{l|ccccc} 
Groups & Pre-control & Pre-ampicillin & Pre-kanamycin & Pre-spectinomycin & Blue marking \\
\hline Pre-ampicillin & 1 & & & & \\
Pre-kanamycin & 0.095 & 1 & & & \\
Pre-spectinomycin & 0.180 & 1 & 0.570 & 1 & 1 \\
Blue marking & 0.180 & 1 & 0.570 & 1
\end{tabular}


In absence of aggressive encounters, the ants presented a probability to survive close to $100 \%$ in groups of 10 ants, aside from the rest of their colony. Marking in blue Argentine ants did not have any significant influence on Argentine ant survival.

\section{Argentine ant aggressive score and survival after marking}

Interactions between unmarked and marked in red Argentine ants as well as between marked in blue and marked in red Argentine ants were non-aggressive (Figure 4.2.a).
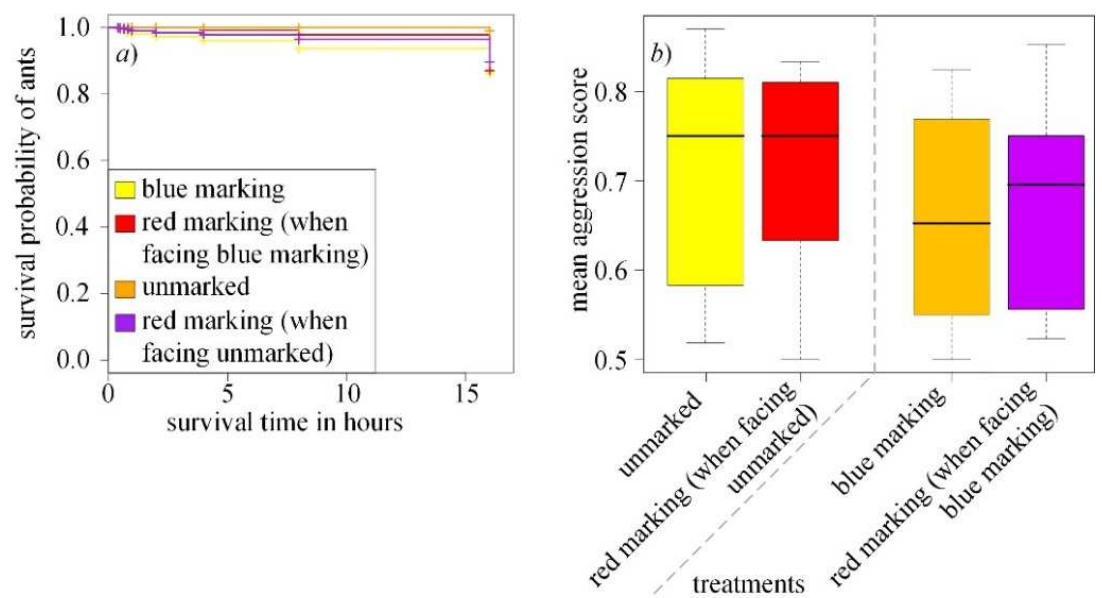

Figure 4.2: a) Mean aggression scores of Argentine ant encounters between marked and unmarked workers; b) Survival probability of unmarked and marked Argentine ants after encounter with marked Argentine ants from the same colony. a) Encounters were realised between ants from the same colony between: unmarked Argentine ants ( $\mathrm{n}=10$ workers $)$ and Argentine ants marked in red ( $\mathrm{n}=10$ workers), and Argentine ants marked in blue ( $\mathrm{n}=10$ workers) and Argentine ants marked in red ( $\mathrm{n}=10$ workers). $b$ ) Argentine ant encounters consisted in unmarked Argentine ants ( $\mathrm{n}=10$ workers) versus Argentine ants with red marking $(\mathrm{n}=10$ workers), and in Argentine ants marked in blue ( $\mathrm{n}=10$ workers) versus Argentine ants with red marking $(\mathrm{n}=10$ workers). For each unmarked / marked pairing, 10 encounters were realised.

The mean aggression scores were all below 2 ( 2 being the first level of aggressive interaction). There were no significance differences in the level of aggression between unmarked, marked in red and marked in blue ants (anova, df $=3$, mean sq $=0.007$, $\mathrm{F}=0.462, \mathrm{p}>0.05)$. Marking ants did significantly decrease the ant survival probability during encounters with Argentine ants from the same colony with a different marking (Likelihood ratio test $=37.9, \mathrm{df}=3, \mathrm{p}<0.001, \mathrm{n}=3600 ;$ coxph) (Figure 4.2.b). Unmarked Argentine ant survival during encounters with Argentine ants marked in red was near $100 \%$ 
when left for $16 \mathrm{~h}$. This result was similar to the survival of Argentine ants in absence of opponents. The survival probability of the encounter between unmarked and marked in red ants was significantly lower for the ants marked in red (coxph, coef $=-3.009$, $\operatorname{se}(\operatorname{coef})=1.025, \exp (\operatorname{coef})=0.0493, \mathrm{z}=-2.937, \mathrm{p}<0.05)$. The survival probability was lower for Argentine ants marked in blue compared to Argentine ants marked in red when encountering them $(\operatorname{coxph}, \operatorname{coef}=-0.689, \operatorname{se}($ coef $)=0.306, \exp (\operatorname{coef})=0.5021, z=-2.25$,

$\mathrm{p}<0.05)$. Argentine ant survival probability when encountering marked in blue was lower than previously observed in absence of any opponents (Figures 4.1 and 4.3). This results may be caused by a negative effect of marking on Argentine ant survival. Perhaps ants may be ingesting paint while grooming themselves and other ants, causing premature death.

\subsubsection{Argentine ant survival in absence of encounters during antibiotic treatments}

Survival of treated Argentine ants in the absence of encounters was close to $100 \%$ for 16 hours during 30 days of the entire antibiotic treatment period (Figure 4.3). 

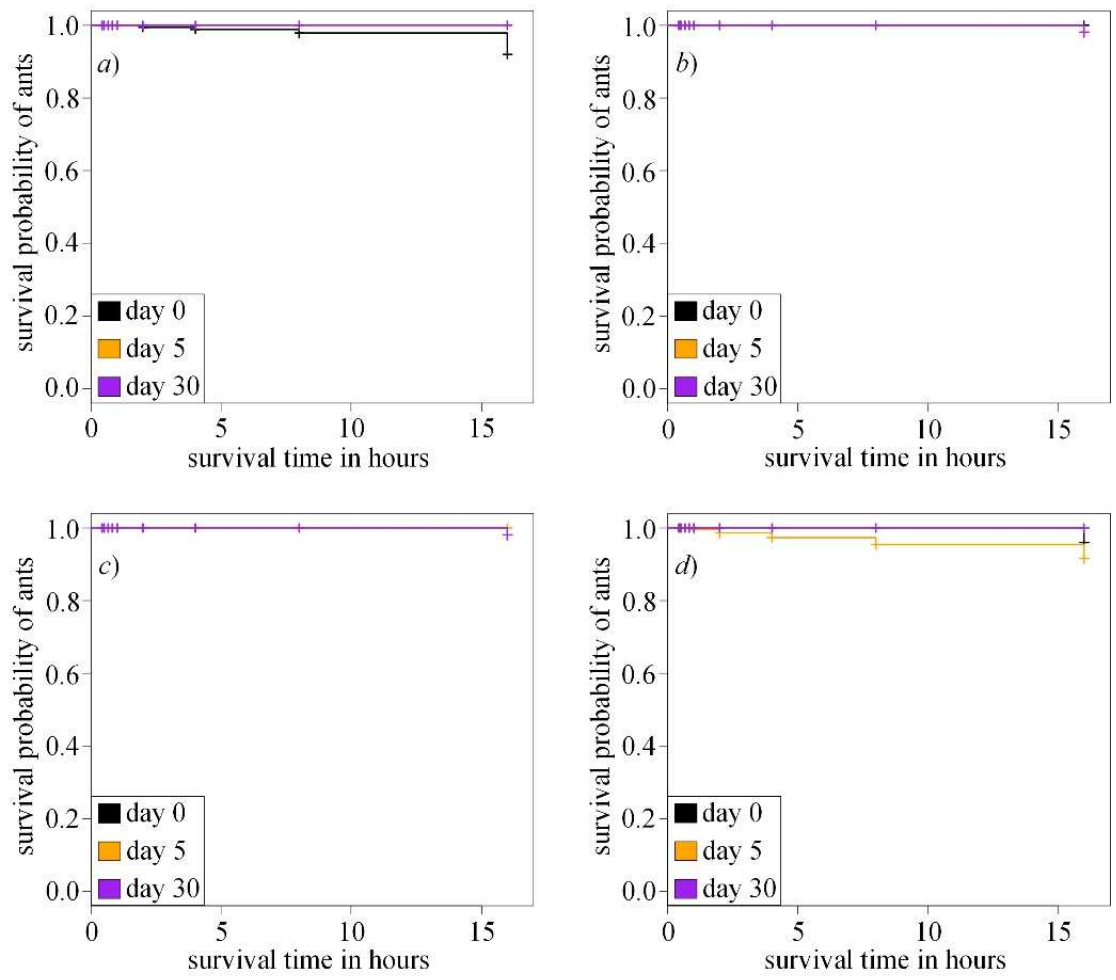

Figure 4.3: Survival probability of treated Argentine ants in absence of encounters at different times (days) in the antibiotic treatment, for a) control, b) ampicillin, c) kanamycin, and d) spectinomycin. Survival probability was observed on groups of ants ( $\mathrm{n}=10$ workers), two pseudo-replicate per treated colony, 10 pseudo-replicates in total per treatment. Survival probability of the treated ants was close to $100 \%$ for all treatments and at each three times (days) tested during the antibiotic treatments.

No significant differences were found between day 0 , day 5 and day 30 of the ampicillin and kanamycin treatments (Table 4.2).

Table 4.2: Likelihood ratio test (coxph) comparison analysis results of survival probability in absence of encounters during antibiotic treatments of Argentine ants. For each treatment, the survival of Argentine ants in the absence of encounters on day 0, day 5 and day 30 of antibiotic treatment were compared. For each day, the survival analysis was realised on two pseudo-replicates per colony $(\mathrm{n}=10$ workers per pseudo-replicate), 10 pseudo-replicates in total per treatment. Significant differences were found for the control and spectinomycin treatments.

\begin{tabular}{l|cccc}
\multicolumn{1}{c|}{ Treatment } & Likelihood ratio test & df & p-value & Number of observations \\
\hline Control & 13.3 & 2 & $\mathbf{p}<\mathbf{0 . 0 5}$ & 1,350 \\
Ampicillin & 1.63 & 2 & 0.443 & 1,350 \\
Kanamycin & 1.63 & 2 & 0.443 & 1,350 \\
Spectinomycin & 13.8 & 2 & $\mathbf{p}<\mathbf{0 . 0 5}$ & 1,350
\end{tabular}


However, significant differences were found between the control and the spectinomycin treatments. The lower survival probability of the control treatment was observed on day 0 , especially after $16 \mathrm{~h}$. These deaths may be caused by dehydration. For spectinomycin, the lower survival probability of the ants could be observed after day 5 of the treatment.

\subsubsection{Argentine ant intra-species aggressiveness and survival during and after antibiotic treatments}

Mean aggression scores of treated Argentine ants and marked non-treated Argentine ants indicated non-aggressive interactions between the ants (Figure 4.4).
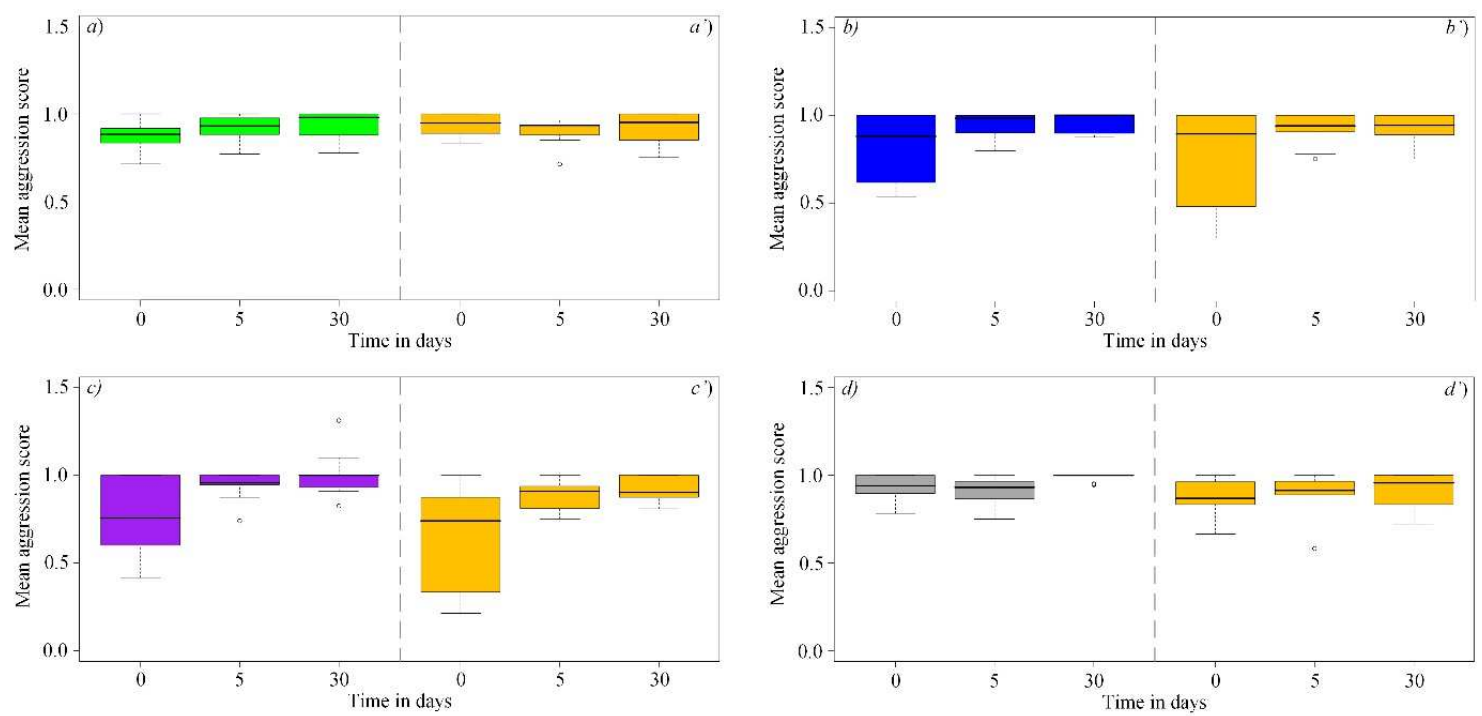

Figure 4.4: Mean aggression scores of ants during inter-species encounters between treated Argentine ants (a) control, b) ampicillin, c) kanamycin, and d) spectinomycin) and marked Argentine ants (a'), b'), c'), and d')). Encounters were realised at three different times during treatment: before treatment (day 0 ), after five days of continuous treatment feeding (day 5), after 30 days of antibiotic treatment (day 30). Encounters corresponded in aggressive interactions between treated Argentine ants ( $\mathrm{n}=10$ workers) and marked Argentine ants ( $\mathrm{n}=10$ workers). Two encounters per Argentine ant colony were realised, for a total of 10 encounters per treatment.

The means of aggression score were all $\leq 1$, meaning that all interactions were nonaggressive. There were significant differences between scores during time for the three antibiotic treatments (Table 4.3). 
Table 4.3: Anova results testing the difference of intra-species aggressiveness of Argentine ants through time for each antibiotic treatment.

\begin{tabular}{l|ccccc} 
Treatment & df & Sum sq & Mean sq & F value & $\operatorname{Pr}(>F)$ \\
\hline Control & 2 & 0.021 & 0.010 & 1.689 & 0.204 \\
Ampicillin & 2 & 0.125 & 0.062 & 4.121 & $\mathbf{p}<\mathbf{0 . 0 5}$ \\
Kanamycin & 2 & 0.284 & 0.142 & 6.306 & $\mathbf{p}<\mathbf{0 . 0 5}$ \\
Spectinomycin & 2 & 0.035 & 0.018 & 4.130 & $\mathbf{p}<\mathbf{0 . 0 5}$
\end{tabular}

On day 5 and day 30 of the treatments, interactions involved non-aggressive physical contact between the ants (inspection with the antennas, score 1) while before treatment (day 0 ) ants would have less physical interactions (ignore, score 0 ). In the control treatment, ants would mainly have non-aggressive physical contacts (Figure 4.3a). Antibiotic treatments did not induce aggressive interactions between treated and non-treated ants.

Survival probability during intra-species encounters was not affected by antibiotic treatments, and was close to $100 \%$ until the end of the antibiotic treatments (Figure 4.5). 

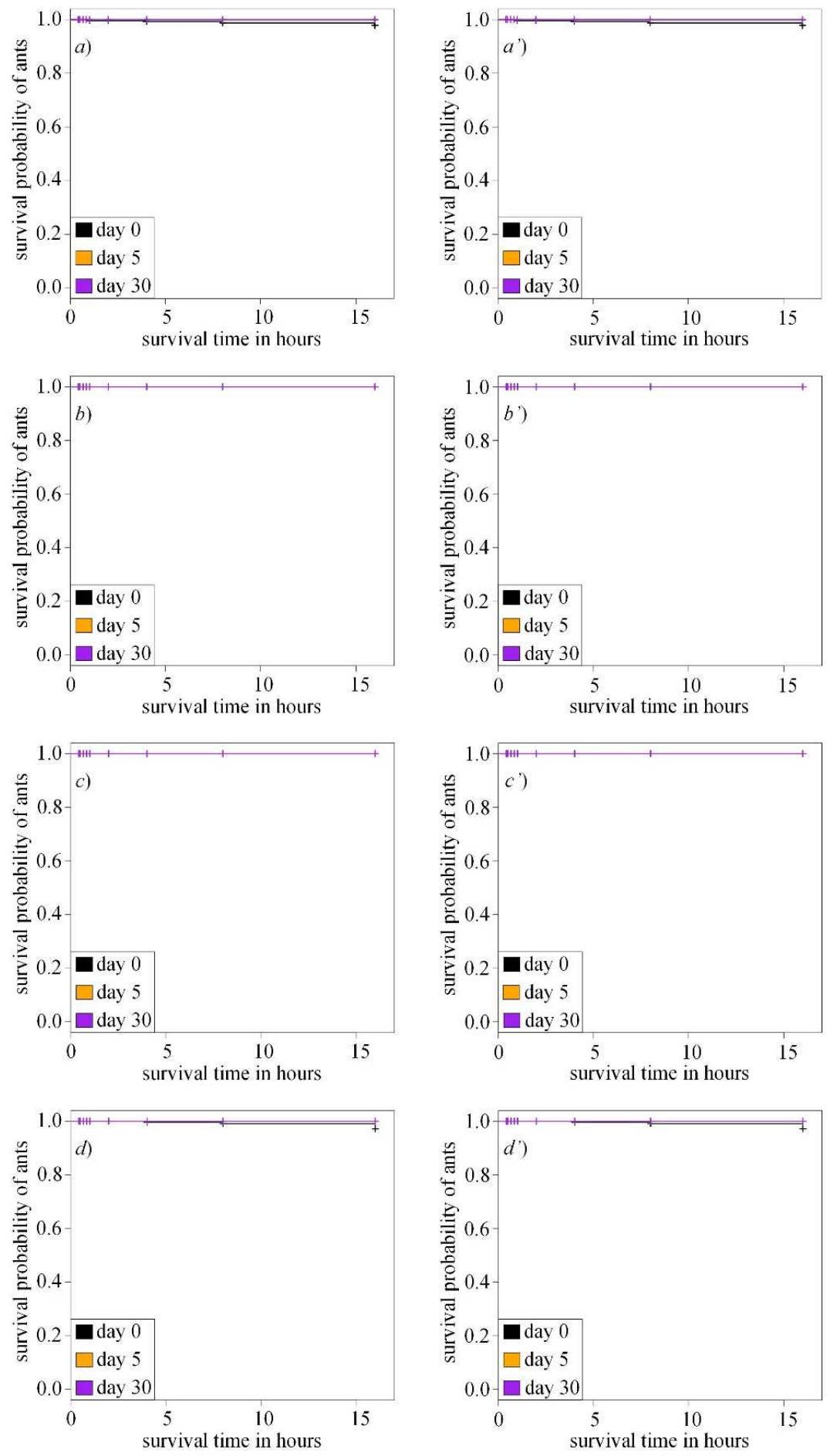

Figure 4.5: Survival probability of intra-species encounters between treated Argentine ants (a) control, b) ampicilling, c) kanamycin, and d) spectinomycin) and non-treated marked Argentine ants (respectively a'), b'), c'), and d')), at three different times during antibiotic treatment. Survival observations were done on two pseudoreplicates encounters per treated colonies, 10 pseudo-replicates in total per treatments. Each encounter was realised with treated Argentine ants ( $\mathrm{n}=10$ workers) and non-treated marked Argentine ants ( $\mathrm{n}=10$ workers). Survival observations were realised before treatment (day 0), after 5 days of treatment (day 5) and after 30 days of treatment (day 30 ). 
However, the difference in survival probability was significantly different for the control, ampicillin and spectinomycin treated ants (Table 4.4).

Table 4.4: Likelihood ratio test (coxph) comparison analysis results of survival probability during intra-species encounters, during antibiotic treatments of Argentine ants. For each treatment, the survival of treated Argentine ants encountering non-treated marked Argentine ants on day 0, day 5 and day 30 of antibiotic treatment were compared. For each day, the survival analysis was realised on two pseudo-replicates per colony ( $\mathrm{n}=10$ workers per pseudo-replicate), 10 pseudo-replicates in total per treatment. Significant differences were found for the control, ampicillin and spectinomycin treatments.

\begin{tabular}{l|cccc} 
Treatment & Likelihood ratio test & df & P-value & Number of observations \\
\hline Control & 11 & 2 & $\mathbf{p}<\mathbf{0 . 0 5}$ & 2,700 \\
Ampicillin & 11 & 2 & $\mathbf{p}<\mathbf{0 . 0 5}$ & 2,700 \\
Kanamycin & 4.08 & 2 & 0.13 & 2,700 \\
Spectinomycin & 8.8 & 2 & $\mathbf{p}<\mathbf{0 . 0 5}$ & 2,700
\end{tabular}

These differences may be caused by dehydration and ingestion of paint by grooming as the ant deaths appeared in majority at the end of the test. The survival probability curves were close to $100 \%$ in all cases.

\subsubsection{Argentine ant inter-species aggressiveness and survival during and after antibiotic treatments}

Mean aggression scores between treated Argentine ants and $P$. advenus were all above score of 2 (Figure 4.6). 

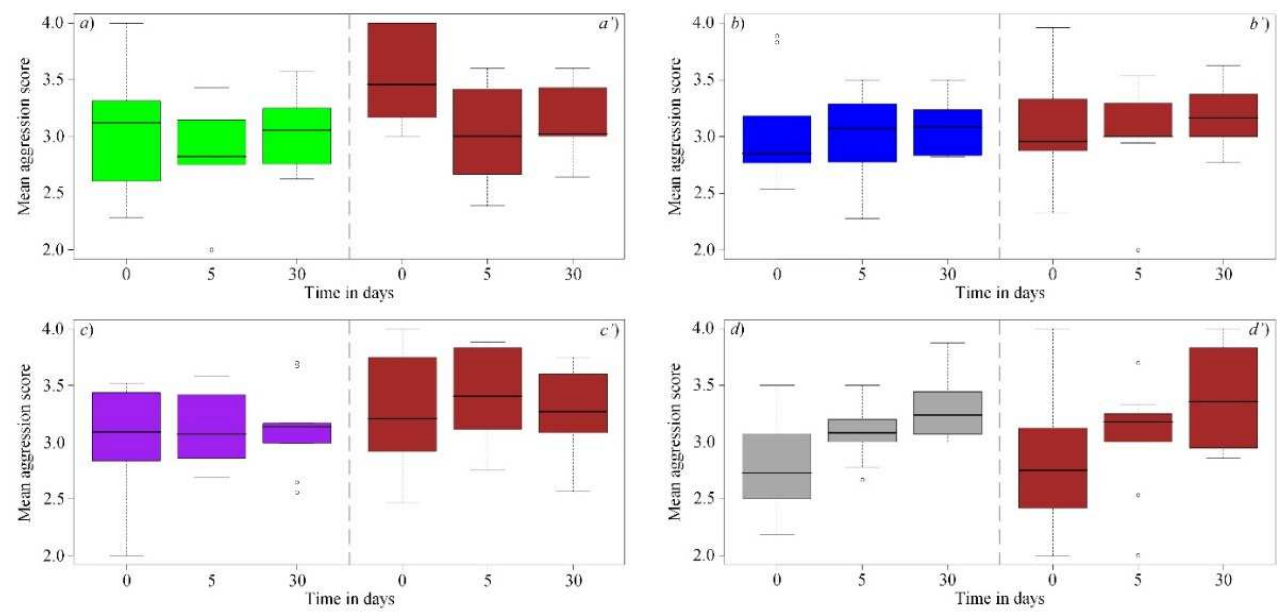

Figure 4.6: Mean aggression scores of ants during inter-species encounters between treated Argentine ants (a) control, b) ampicillin, c) kanamycin, and d) spectinomycin) and Prolasius advenus (a'), b'), c'), and d')). Encounters were realised at three different times during treatment: before treatment (day 0), after five days of continuous treatment feeding (day 5), after 30 days of antibiotic treatment (day30). Encounters corresponded in aggressive interactions between treated Argentine ants ( $\mathrm{n}=10$ workers) and P. advenus ( $\mathrm{n}=10$ workers). Two encounters per Argentine ant colony were realised, for a total of 10 encounters per treatment.

Encounters between treated Argentine ants and $P$. advenus were all aggressive. The aggression level by treated Argentine ants toward $P$. advenus did not significatively change during the antibiotic treatments except for spectinomycin (Table 4.5).

Table 4.5: Anova results testing the difference of inter-species aggressiveness of Argentine ants through time, for each antibiotic treatment.

\begin{tabular}{l|lllll} 
Treatment & df & Sum sq & Mean sq & F value & $\operatorname{Pr}(>\mathrm{F})$ \\
\hline Control & 2 & 0.301 & 0.150 & 0.868 & 0.431 \\
Ampicillin & 2 & 0.038 & 0.019 & 0.134 & 0.875 \\
Kanamycin & 2 & 0.024 & 0.012 & 0.081 & 0.923 \\
Spectinomycin & 2 & 1.261 & 0.6304 & 6.621 & $\mathbf{p}<\mathbf{0 . 0 5}$
\end{tabular}

Spectinomycin treated ants significantly increased their aggression level over time during the antibiotic treatment (Figure 4.6). In response, $P$. advenus ants increased their aggression levels (Figure 4.5). High level of aggression diminished the survival probability during a 17 hour inter-species encounter for both Argentine ants and $P$. advenus, for all treatments (Figure 4.7). 
Treatments vs Argentine ants
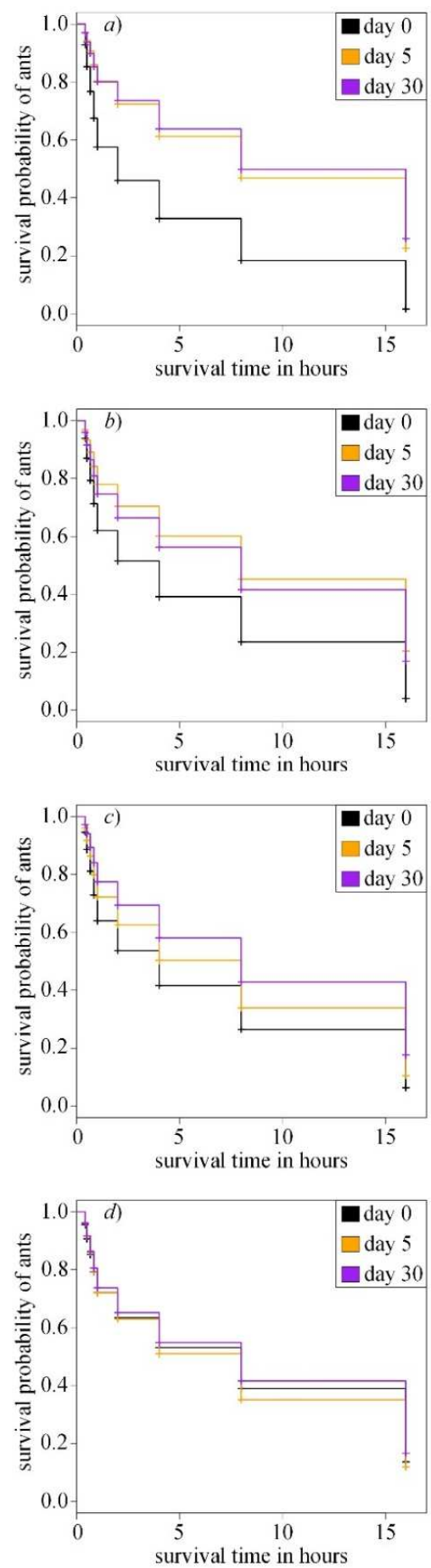

Treatments vs $P$. advenus
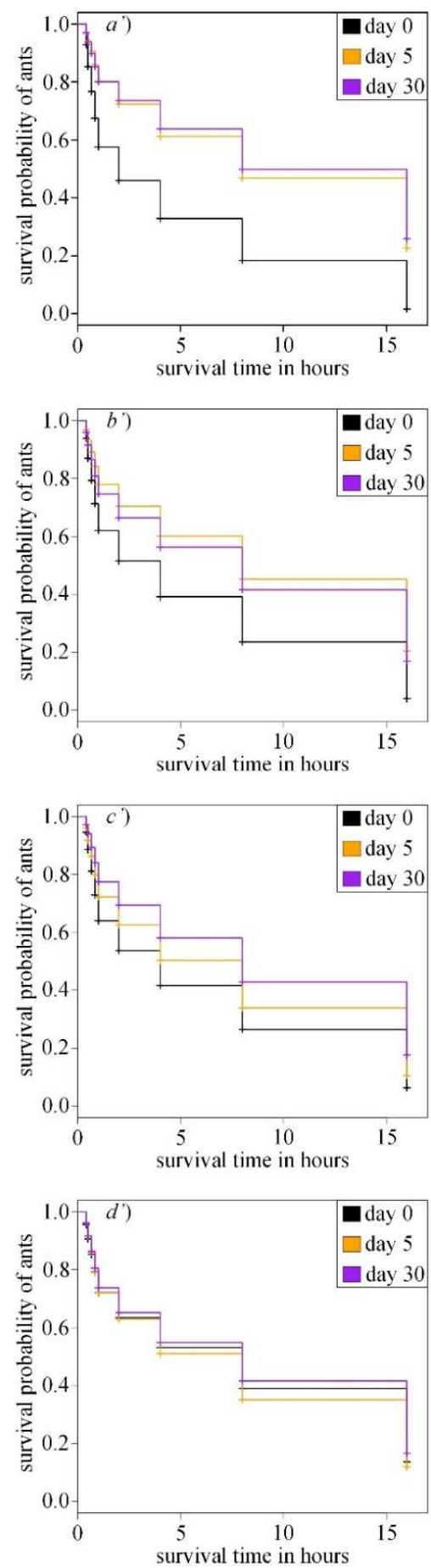

Figure 4.7: Survival probability of inter-species encounters between treated Argentine ants ((a) control, b) ampicillin, c) kanamycin, and d) spectinomycin) and Prolasius advenus (respectively a'), b'), c'), and d')), at three different times during antibiotic treatment. Survival observations were done on two pseudo-replicates encounters per treated colonies, 10 pseudo-replicates in total per treatments. Each encounter was realised with treated Argentine ants ( $\mathrm{n}=10$ workers) and $P$. advenus ( $\mathrm{n}=10$ workers). Survival observations were realised before treatment (day 0), after 5 days of treatment (day 5 ) and after 30 days of treatment (day 30 ). 
Survival probability decreased from nearly $100 \%$ in absence of aggressive encounters (Figure 4.1) to below 30\% during a 16 hour inter-species encounters for all treatments. The survival probability of treated Argentine ants was significantly different over time during the antibiotic treatments for the control, ampicillin and kanamycin treatments, but not for spectinomycin (Table 4.6).

Table 4.6: Likelihood ratio test (coxph) comparison analysis results of survival probability during inter-species encounters, during antibiotic treatments of Argentine ants. For each treatment, the survival of treated Argentine ants encountering Prolasius advenus on day 0 , day 5 and day 30 of antibiotic treatment were compared. For each day, the survival analysis was realised on two pseudo-replicates per colony $(n=10$ workers per pseudo-replicate), 10 pseudo-replicates in total per treatment. Significant differences were found for the control, ampicillin and kanamycin treatments.

\begin{tabular}{l|cccc} 
Treatment & Likelihood ratio test & df & p-value & Number of observations \\
\hline Control & 258 & 2 & $\mathbf{p}<\mathbf{0 . 0 5}$ & 2,700 \\
Ampicillin & 105 & 2 & $\mathbf{p}<\mathbf{0 . 0 5}$ & 2,700 \\
Kanamycin & 53.6 & 2 & $\mathbf{p}<\mathbf{0 . 0 5}$ & 2,700 \\
Spectinomycin & 3.45 & 2 & 0.178 & 2,700
\end{tabular}

Overall, the control, ampicillin and kanamycin treatments presented a high level of aggressive interactions during inter-species encounters. Survival probability increased over time for the control and kanamycin treatments (Figure 4.6). For ampicillin, the survival probability increased after 5 days and was similar to day 5 on day 30 of the treatment (Figure 4.6).

\subsubsection{Argentine ant survival and fitness after antibiotic treatments}

There were no differences in the brood production based on treatments and the number of queens (Table 4.7). 
Table 4.7: Anova analysis results for the difference in fitness (brood production) between antibiotic treatments. Each treatment (control, ampicillin, kanamycin and spectinomycin) was given to five colonies during 30 days. For each colony, the brood and number of queens was counted at the end of the antibiotic treatment. Brood production was considered representative of the fitness of the colonies. No significant differences of fitness were observed considering the treatment and number of queens.

\begin{tabular}{l|ccccc}
\multicolumn{1}{c|}{ Factors } & df & Sum square & Mean square & F value & $\operatorname{Pr}(>\mathrm{F})$ \\
\hline treatment & 3 & 15,498 & 5,166 & 0.223 & 0.880 \\
number of queens & 1 & 1,411 & 1,411 & 0.061 & 0.806
\end{tabular}

Argentine ant survival probability of colonies declines during the treatments (Figure 4.8).

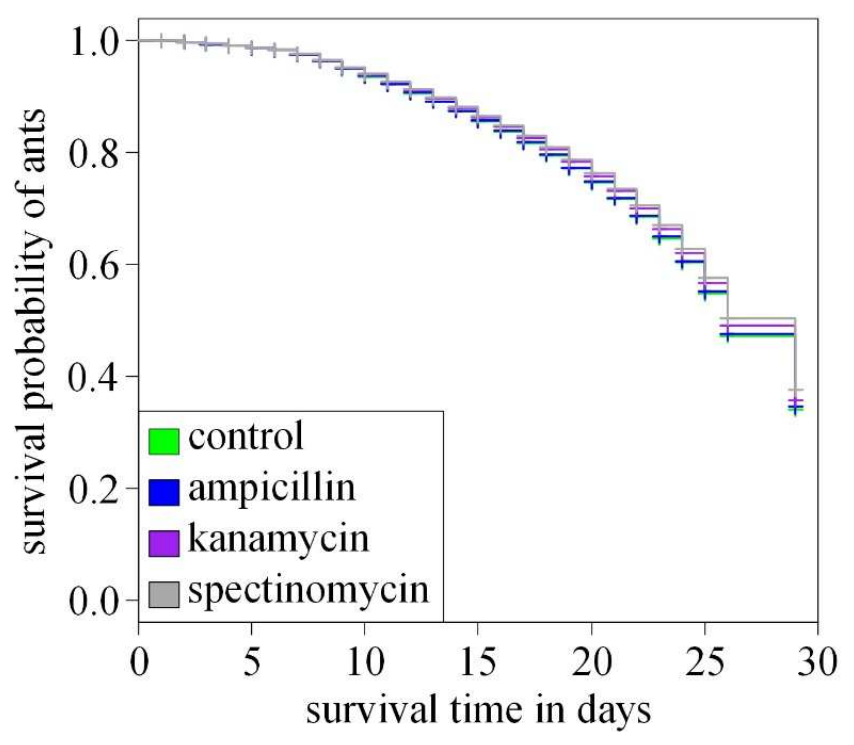

Figure 4.8: Argentine ant colony survival probability during antibiotic treatments. Treatments were given every day during five consecutive days (from day 2 to day 9), and then three times a week during three weeks. The number of ants was counted daily from day 1 to day 26 of the experiment and one final time on day 29. The same number of ants were also taken from every colony on day 2, 7, 8 and 9 for aggressive encounter interactions and survival tests.

The decline seemed likely to be caused by workers taken from the colony for aggressive encounters and survival analysis. A total of 140 ants were retrieved from each colony during antibiotic treatments. Differences between survival probability of the treated colonies were significant (Likelihood ratio test $=84.3, \mathrm{df}=3, \mathrm{n}=342,630, \mathrm{p}<0.05$; $\operatorname{coxph}$ ). Every treatment differed significantly from each other (coxph, multiple reorder of factors, in every case $\mathrm{p}<0.05)$. Survival probability was the lowest for the control, and increased in 
order from ampicillin, kanamycin and was the highest for spectinomycin. Antibiotic treatments seem to improve colony survival.

\subsection{Discussion}

As predicted, the antibiotic treatments did increase Argentine ant colony survival. However, they did not affect the ant's fitness. While antibiotics treatments did not lead to any changes in Argentine ant intra-aggression levels, spectinomycin treatments did increase the ant inter-species aggressiveness.

\section{Argentine ant intra-species aggression level and survival did not change during antibiotic treatments}

Argentine ants did not present any aggressive behaviour towards other Argentine ants, even if the ants were from two colonies separated from each other for multiple months. In New Zealand, Argentine ants constitute one single population (Corin et al., 2007a). Workers from nests geographically separated do not aggress each other (Corin et al., 2007a). Under laboratory conditions, Argentine ants fed different diets present some aggressive behaviour toward other Argentine ants (Liang and Silverman, 2000; Corin et al., 2007a). The absence of aggressive interactions between treated and non-treated Argentine ants indicates that the change of bacterial diversity cause by antibiotics did not affect Argentine ant nestmate recognition. Argentine ant nestmate recognition is based on the ant cuticular hydrocabones (Liang and Silverman, 2000). Cuticular hydrocarbon signature depends upon the ant genetics but also diet (Liang and Silverman, 2000; Liang et al., 2001). Similarily, bacterial diversity does not seems to be involved in Argentine ant nestmate recognition at the difference of Reticulitermes speratus termites (Matsuura, 2001).

\section{Argentine ant inter-species aggression level and survival mixed results during antibiotic} treatments

Treated Argentine ants presented a high level of aggression toward Prolasius advenus, an new ant opponent, and a low survival probability during the inter-species encounters. The 
high level of aggression and low survival probability during inter-species interaction was expected. Among the seven ants included in the list of the worst global invaders, Argentine ants are one of the most aggressive invasive ants, with a low survival probability and high killing rate (Bertelsmeier et al., 2015). Argentine ants react aggressively to other ant species in order to defend their nests and their food sources, and may die in the process (Bertelsmeier et al., 2015).

Aggression levels toward an unknown ant species did not change over time except for spectinomycin treated ants. The change in bacterial diversity caused by ampicillin and kanamycin did not seem to affect Argentine ant aggression levels towards another ant species. However, spectinomycin did affect bacterial diversity so that the ants were more aggressive toward unknown ants. Increase in inter-species aggression may be caused by the change of bacterial diversity and the stress induced by spectinomycin treatments. The increase in aggressiveness may explain the low survival probability of spectinomycin treated ants through the antibiotic treatment.

Survival during inter-species interactions improved over time but for spectinomycin treated ants. The improved survival between day 0 and day 5 may be due to a lower stress in Argentine ants. Argentine ant workers were counted one day prior to day 0 encounters. In addition, ants were not feed prior to the antibiotic treatments. The stress associated with the lack of food may have lowered Argentine ant endurance and lowered their survival probability in stressful inter-species encounters. Between day 0 and day 5, the ants were all feed and were not disturbed. Therefore, the survival probability increase between day 0 and day 5 for the control, ampicillin and kanamycin treatments may be due to a lower stress and the well fed state of the ants. Furthermore, the absence of survival probability increase for spectinomycin treated ants may be caused by their increase aggressiveness. Physical aggression levels increased and may lead to a higher death rate.

\section{Argentine ant colony fitness during and after antibiotic treatments}

Antibiotic treatments, ampicillin, kanamycin and spectinomycin, seem to improve Argentine ant colony survival but not affect the colony fitness. Antibiotics modify bacterial diversity present in ants (see Chapter 3). This change in bacterial diversity may promote the ant health and increase the ant survival. Bacterial diversity has indeed been associated with host health. For honey bees (Apis spp.), Lactobacillus and other gut bacteria are 
involved in their host health and immune system. The bacteria prevent bacterial, viral and fungal infections. In the case of Argentine ants, antibiotic treatments may have decreased pathogenic bacteria present in the ants. Favourable opportunistic bacteria may have increased their population and indirectly promote their host survival.

The absence of effects of the antibiotics on fitness may be due to the low exposition of the queens to the antibiotics. Forager ants retrieve the food for the colony, ingesting high amounts of antibiotics while doing so. Foragers then share the food by trophallaxis, mouth to mouth, with nestmates, including nurse ants. Nurses are the ones feeding the queens and the larvae. This chain of food transmission may cause a difference in antibiotic exposure, high for foragers and low for queens and brood. This low quantity of antibiotic exposure may not have affect the queens.

\section{Critical evaluation of experimental procedures}

Ideally, Chapter 3 and Chapter 4 would have been realised on the same Argentine ant colonies. Using 454 technology to analyse the change in bacterial diversity during antibiotic treatments at day 0 , day 5, day 30 and at the end of the two months experiment would have offered an insight on how bacterial diversity was affected by the antibiotic treatments. This change of bacterial diversity may then have been associated with the observed modifications in inter-species encounter aggressiveness level and survival. However, time constrains associated with limitations of 454 sequencing did not allow it.

Another possibility would have been the use of specific primers and qPCR in order to detect the change in presence and activity of specific bacteria. Bacteria would have been selected from 454 sequencing after antibiotic treatment from Chapter 3 results. Bacteria presence at

day 0 , day 5 , day 30 and at the end of the two months experiment would have offered an insight in the change of bacterial diversity during and after antibiotic treatment. However, kanamycin and spectinomycin treated ants were not sequenced in Chapter 3 because of 454 sequencing constrains.

The use of a different type of marking to differentiate treated ants from non-treated ants may not have affected marked Argentine ant survival and Argentine ants interacting with them. However, marking was needed to differentiate the ants. Already the colour blue was selected as the red marking seemed to cause a high death rate during marking (personal observation). 
Finally, as for Chapter 3, the direct contact of queens with the antibiotics may have offered a different colony survival and fitness results. However, access to the queen was difficult during the experiment, and may have caused additional stress. A different experiment focussing on queens may be possible: separating them from the nest, placing them with a few workers for support and feeding them antibiotics during a few days before placing them back in the colony. In addition, 454 sequencing of the bacteria present in the queens before and after treatment may inform on the effects of antibiotic feeding and the change in bacterial diversity. The change or absence of change in bacterial diversity may then be associated to the observed colony fitness.

Antibiotic treatments modify the bacterial diversity present in Argentine ants. As a consequence, Argentine ant colony survival increased. However, the ant fitness did not seem to be affected. Furthermore, the change in bacterial diversity did not modify intraspecies interactions. This result indicates that intra-species recognition may not have been modified by the change of bacterial diversity caused by antibiotic treatments. Inter-species aggression level increased for one treatment, spectinomycin. Bacterial diversity may therefore influence Argentine ant aggression level with other species and at length influence the behaviour dominance status of the ants. The change in bacterial diversity present in invasive ants may be involved in invasive species population dynamics and aggression behaviour. However, population dynamics are complex and may also involve pathogenic viral infections. A first detection of viruses in Argentine ants may allow the discovery of additional potential factors involved in the reported Argentine ant population decline in New Zealand (see Chapter 5). 
Chapter 5. Virus discovery in the invasive Argentine ants 


\subsection{Abstract}

Viruses have been extensively studied in social insects such as in honey bees to better understand colony collapse disorder (CCD). Viruses have also been discovered in red imported fire ants (Solenopsis invicta) and are potential bio-controls. Viruses appear to offer potential understanding in the population declines of invasive social insects. There is no knowledge on viruses infecting the invasive Argentine ant (Linepithema humile). This study aimed to identify for the first time viruses infecting Argentine ants as a first step towards a better understanding of Argentine ant population declined in New Zealand and to highlight the potential viruses introduced by invasive species. To examine viruses, I used Illumina sequencing from one sample of Argentine ants in New Zealand and RT-PCRs in samples from the ant home range (Argentina) and two invaded countries (Australia and New Zealand). From the next generation sequencing data, I identified by BLASTx searches on GenBank a new dicistrovirus, tentatively named L. humile virus -1 (LHUV-1). LHUV1 actively replicates within the ants, and was found in all countries and sites but one site in New Zealand. Using published primers, Deformed wing virus (DWV) was found to infect Argentine ants in two of the three sites in Argentina and a different replicating DWV strain in 21 of the 27 sites in New Zealand. No infection by the bee viruses Israeli acute paralysis virus, Kashmir bee virus or Acute bee paralysis virus nor by the ant viruses Solenopsis invicta virus -1 and -2 were detected in any of the three countries. The infection by DWV, a bee virus associated with CCD, and LHUV-1, a dicistrovirus, may be a potential cause of the population decline of Argentine ants in New Zealand. Furthermore, Argentine ants may form potential reservoirs for the spread of DWV and LHUV-1 to native species and domesticated honey bees in New Zealand. Additional studies on the effects of these two viruses on Argentine ant fitness will give a better understanding on the ant population fluctuations in invaded countries. If specific to Argentine ants, LHUV-1 may be a first step in the development of a bio-control for the ants. 


\subsection{Introduction}

\section{Invasive species and co-introduction of viruses}

Invasive species represent a global threat that challenges economies (Pimentel et al., 2005; Cook et al., 2007), due their negative impact on agriculture (Perrault et al., 2003) and human health (Mazza et al., 2013). Furthermore, invasive species can have disastrous longterm effects on native ecosystems. Invasive species are also known to displace native animal populations and decrease native plant fitness through mechanisms such as predation, food competition and invasional meltdown (Mooney and Cleland, 2001; O’Dowd et al., 2003; Blancafort and Gómez, 2005; Grosholz, 2005; Yang et al., 2012).

In addition, invasive species can co-introduce harmful pathogens including viruses that can potentially infect native species by spillover (De Barro et al., 2008; Kojima et al., 2011; Strauss et al., 2012; Arbetman et al., 2013; Levitt et al., 2013). In the worldwide introduced honey bees (Apis mellifera), viruses infecting multiple apiaries, such as the Deformed wing virus (DWV), Israeli acute paralysis virus, Kashmir bee virus, and the Acute bee paralysis virus, are associated with honey bee colony collapse disorder (Stankus, 2008; Genersch, 2010b; Evans and Schwarz, 2011). Some of these bee viruses can also be found in other arthropod species, including ants, foraging in the vicinity of infected beehives (Celle et al., 2008; Singh et al., 2010; Levitt et al., 2013). Cross-species transmission of viruses from invasive species to native ones can occur by direct interaction between species (Eyer et al., 2009), and by indirect interaction, such as from foraging on contaminated pollen (Singh et al., 2010; Mazzei et al., 2014) or because of mite infections (Shen, 2005). The effects of the introduced viruses on native species are not always known or in some cases hasvebeen only tested in laboratories (Meeus et al., 2014).

\section{Invasive species as new reservoir for local viruses}

Invasive species can themselves become new reservoirs for diseases already present in their new environments. If viruses actively replicate within the new hosts, native species can later be infected by spillback (Marsot et al., 2011, 2013). The invasive house sparrows (Passer domesticus) became a new host for the Buggy Creek virus in its new environment in Nebraska. In turn, the high proportion of infected house sparrow increases the infection 
probability of native cliff swallows (Petrochelidon pyrrhonota) (O'Brien et al., 2011). Furthermore, the infection of invasive species as additional hosts for the native viruses can lead to the selection of more virulent strains. DWV is a bee (Apis spp.) virus that can be transmitted by invasive parasitic mites (Varroa destructor) (de Miranda and Genersch, 2010). More virulent strains are infecting bees when DWV is transmitted from one infected bee to another bee via mites (Ryabov et al., 2014).

In the specific case of invasive social insects, the high number of workers and large colony size can facilitate the spread of introduced and/or native pathogens through spillover and spillback mechanisms, increasing the pressure on native species. Therefore, the identification of viruses present in invasive social insects will help understand better the potential pathogenic threats native species are facing. These pathogenic viruses may also affect invasive species populations and induce a population decline of the invasive species.

\section{Viruses nomenclature and active replication}

Next-generation sequencing technology allows the discovery of multiple new viruses. While new information on viruses is made available, virus nomenclature stays complex (Adams et al., 2013). Viruses have short sequences and a high mutation rate compared to other organisms, especially with RNA viruses. These particularities create challenges in the classification and identification of viruses. However, conserved genes such as those coding for the polymerase enzyme and for structural proteins can be used in order to identify and classify viruses. These genes are under selection pressure and therefore present a lower mutation rate. Viruses from the same families typically present similar sequences of their coding genes while differing at a comparatively high rate for non-coding sections of their genomes. The detection of potential coding genes is therefore important in order to identify or determine if a virus is taxonomically new to science.

Once a virus is detected in an organism, information about the activity of the virus can be gathered by testing its possible active replication. An analysis of replication enables us to determine if there is evidence that the host is parasitized by the virus, or alternatively if there is no replication the virus may have been present by other pathways such as being in ingested food. In the case of positive strand RNA viruses, replication of the virus can be assessed by reverse-transcriptase PCR (RT-PCR) targeting the virus negative strand. If a 
positive strand RNA virus is active, negative strand of the virus (negative strand of RNA) will be detected in the infected host.

\section{The case of the invasive Argentine ant}

Argentine ants were introduced in New Zealand from Argentina after a complex invasion history (see Chapter 1, section 1.8). It is possible that Argentine ants may have cointroduced some viruses from their home range into their new environments. In addition, Argentine ants may have been infected by new viruses between each invasion event. These co-introduced viruses present a potential threat to native species interacting directly or indirectly with the ants. Furthermore, part of the Argentine ant population in New Zealand underwent an unexplained decline (Cooling et al., 2012). As some viral infections are known to be a cause of population decline in other species (Valles et al., 2004, 2014;

Highfield et al., 2009; Evans and Schwarz, 2011), investigating the presence of viruses in Argentine ants in New Zealand may offer an insight into the possible role of pathogens in the observed population collapse. To the best of our knowledge, no previous studies have been carried out to record or examine viruses infecting Argentine ants.

\section{Chapter aims}

This study aimed to examine and identify for the first time DNA and RNA viruses present in Argentine ants using Illumina sequencing. It was hypothesised that: 1) Argentine ants are the hosts of pathogenic viruses; 2) Argentine ants are infected by viruses in both their home range and invasive rage.

\subsection{Materials and methods}

\subsubsection{Metagenomic discovery of viruses}

\section{Sample collection, preparation and sequencing}

Argentine ant workers were collected alive in February 2013 with an aspirator from two close nests $\left(\sim 500\right.$ ants from each nest), in Wellington, New Zealand $\left(41.221893^{\circ} \mathrm{S}\right.$, $174.87241^{\circ} \mathrm{E}$; and $\left.41.223118^{\circ} \mathrm{S}, 174.872731^{\circ} \mathrm{E}\right)$. 
For each of the two nests, one pool of 30 ants was ground up using pestles in microcentrifuge tubes containing $400 \mu \mathrm{L}$ iPrep PureLink Virus kit lysis buffer (Life Technologies, Carlsbad, CA, United States). Water $(400 \mu \mathrm{L})$ and $50 \mu \mathrm{L}$ proteinase $\mathrm{K}$ was then added to each sample and incubated at $50^{\circ} \mathrm{C}$ for $1 \mathrm{hr}$, followed by $96^{\circ} \mathrm{C}$ for $5 \mathrm{~min}$. Samples were then centrifuged at 12,000 x g for $5 \mathrm{~min}$. The supernatant was removed and the extraction was undertaken using iPrep Purelink Virus kit (Life Technologies, Carlsbad, CA, United States). The nucleic contents were eluted into $50 \mu \mathrm{L}$ of RT-PCR molecular grade water (Ambion, Austin, TX, United States). Duplicate nucleic acid samples were then combined into a single sample to maximise the amount of nucleic acid that was extracted. DNA and RNA were co-purified during the extraction. In order to detect RNA viruses, DNA was removed by treatment with DNAse using Ambion DNA-free kit (Life Technologies, Carlsbad, CA, United States) and $8 \mu \mathrm{L}$ of DNA-free RNA was used as template for cDNA synthesis (Life Technologies, Carlsbad, CA, United States) and RNase $\mathrm{H}$ digestion. The DNase digestion step was not required for the complementary approach to find DNA viruses. To ensure that the minimum requirement of $1 \mu \mathrm{g}$ of DNA for library preparation was obtained, DNA and cDNA was amplified using multiple displacement amplification, using the Whole Transcriptome Amplification kit (Qiagen, Valencia, CA, United States). Amplified DNA libraries were then prepared using the Illumina TruSeq DNA library preparation kit followed by sequencing on an Illumina MiSeq instrument producing 250 bp paired-end reads (New Zealand Genomics Limited, Massey Genome Service, Massey University, Palmerston North, New Zealand).

\section{Bioinformatics}

The quality of the sequence data was checked using Fast QC. The total read length of $250 \mathrm{bp}$ was trimmed when the average quality score was less than 30. Duplicate reads were collapsed using FASTX-Toolkit (version 0.10.1). Velvet (version 1.2.07) (Zerbino and Birney, 2008) was used for de novo assembly of the trimmed sequence data with a k-mer of 75. Contigs were compared to the GenBank non-redundant nucleotide sequence database using BLASTn from BLAST+ (version 2.2.27) and an e-value threshold for reporting of 0.001. BLAST outputs were visualised in MEGAN (version 4.7) (Huson et al., 2007) for taxonomic assignment. The coding or non-coding role of the sequences was assumed from the taxonomic assignment results and four contigs of interest (i.e. n6409, n1000, n1050 and n1905) were selected (see section 5.4). 


\section{Confirmatory RT-PCR}

RNA viral sequences of interest from the metagenomic data were confirmed using specific RT-PCR assays on the original RNA extraction. Primers were designed using Geneious 6.1.7 (Kearse et al., 2012), Primer3Plus (Untergasser et al., 2007, 2012) and AmplifX 1.7.0 (Jullien - http://crn2m.univ-mrs.fr/pub/amplifX-dist) to target one selected non-coding contig n1905 and one coding contig n9293 (Table 5.1).

Table 5.1: List of designed primers specific to selected RNA potential viral contig sequences found in Argentine ant after preliminary Illumina sequencing. Potential RNA virus contigs $s^{\mathrm{a}}$ are considered non-coding sequences and potential RNA virus contigs ${ }^{\mathrm{b}}$ coding sequences, after BLASTn best hit match analysis.

\begin{tabular}{|c|c|c|c|c|}
\hline $\begin{array}{l}\text { Potential RNA } \\
\text { virus contigs }\end{array}$ & Primer name & $\begin{array}{c}\text { Primer } \\
\text { orientation }\end{array}$ & Primer sequence $\left(5^{\prime}\right.$-> $\left.3^{\prime}\right)$ & $\begin{array}{c}\text { Product } \\
\text { length (bp) }\end{array}$ \\
\hline \multirow{2}{*}{$\mathrm{n} 1000^{\mathrm{a}}$} & n1000-P1F & Forward & GGGGTTGTGATACGTCTGCT & \multirow{2}{*}{707} \\
\hline & n1000-R1R & Reverse & GGTGTCGAGGTGGACTTGAT & \\
\hline \multirow{2}{*}{ n1905 a } & n1905-P1F & Forward & GTTTGGCATTGACGGAGAAT & \multirow{2}{*}{538} \\
\hline & n1905-P1R & Reverse & TTTCCTGAGGGTTGGCTATG & \\
\hline \multirow{2}{*}{$\mathrm{n} 1050^{\mathrm{a}}$} & $116 \mathrm{~F}$ & Forward & AGTGCTTTGGCTCCTGCATT & \multirow{2}{*}{592} \\
\hline & $706 \mathrm{R}$ & Reverse & TTATTGGCGCAGGCTTGATG & \\
\hline \multirow{2}{*}{$\mathrm{n} 2445^{\mathrm{b}}$} & $\mathrm{n} 2445-\mathrm{G} 131 \mathrm{~F}$ & Forward & TATCCGGGAGTCGAACTCGA & \multirow{2}{*}{153} \\
\hline & n2445-GA280R & Reverse & TGAGGGTCCTAGGACTGGTG & \\
\hline \multirow{2}{*}{ n6399 } & n6399-G95F & Forward & GGTCGTCAACTATCGGTTTGTCGT & \multirow{2}{*}{348} \\
\hline & n6399-GA442R & Reverse & TCTGTTCAAACCCAGGCGTTAAGG & \\
\hline \multirow{2}{*}{ n6409 } & n6409-A656F & Forward & AGGAAACGCAGCCAGTGCTATT & \multirow{2}{*}{400} \\
\hline & n6409-A1055R & Reverse & GTCACCTGACTCCTTGCCTGATTT & \\
\hline \multirow{2}{*}{ n16939 } & n16939-A11F & Forward & GCTACGACACCCCTTAACTGCTTT & \multirow{2}{*}{202} \\
\hline & n16939-GA212R & Reverse & AGGCCTCTGCGATAGTAGACA & \\
\hline \multirow{2}{*}{ n9293 } & n9293-contig-cap-G3F & Forward & TTCCAACTCGTCGGCTAGAC & \multirow{2}{*}{370} \\
\hline & n9293-contig-cap-G372R & Reverse & TTCCAACTCGTCGGCTAGAC & \\
\hline
\end{tabular}

One step RT-PCR assays were performed using a SuperScript III One Step RT-PCR system with Platnium Taq DNA polymerase (Life Technologies) following the modified protocol described here. A total reaction volume of $25 \mu \mathrm{L}$ composed of: $\sim 20 \mathrm{ng}$ RNA, 1X Reaction mix, $0.8 \mu \mathrm{M}$ of each primer, $5.5 \mu \mathrm{L} \mathrm{ddH}_{2} \mathrm{O}$ and $1 \mu \mathrm{L}$ SuperScript ${ }^{\circledR}$ III RT/Platinum ${ }^{\circledR}$ Taq Mix was prepared. Thermal cycling parameters were as follows: reverse transcription at $45^{\circ} \mathrm{C}$ for $30 \mathrm{~min}$, then an initial denaturation step at $94^{\circ} \mathrm{C}$ for $2 \mathrm{~min}$, followed by 35 cycles of $94^{\circ} \mathrm{C}$ for $15 \mathrm{sec}, 55^{\circ} \mathrm{C}$ for $15 \mathrm{sec}$ and $68^{\circ} \mathrm{C}$ for $30 \mathrm{sec}$, with a final extension step of $68^{\circ} \mathrm{C}$ for 5 min. Amplicons were visualised by gel electrophoresis on a 1.5\% agarose gel stained 
with ethidium bromide. Amplicons were purified using USB ExoSAP-IT (Affymetrix) and sent for Sanger sequencing on a capillary sequencer to Massey University (Genome Service, Palmerston North, New Zealand). Sanger sequences were then examined for quality in MEGA6 and aligned with ClustalW (default parameters) to their original contig sequences to confirm the Sanger sequence identity.

\subsubsection{Distribution of Argentine ant viruses within New Zealand and}

\section{Australia}

\section{Sample collection}

Argentine ant workers were collected in $100 \%$ ethanol or in Ambion RNAlater (Life Technologies) between 2012 and 2015 from 32 sites distributed among 9 cities/regions covering the invasive ant's range in New Zealand, two sites in Australia and three sites in the ant home range Argentina (Figure 5.1, details in Appendix IV). Samples were preserved at $-20^{\circ} \mathrm{C}$ except for the Australian samples stored at $4^{\circ} \mathrm{C}$ and one sample in New Zealand (Gisborne) snap froze and conserved at $-80^{\circ} \mathrm{C}$.

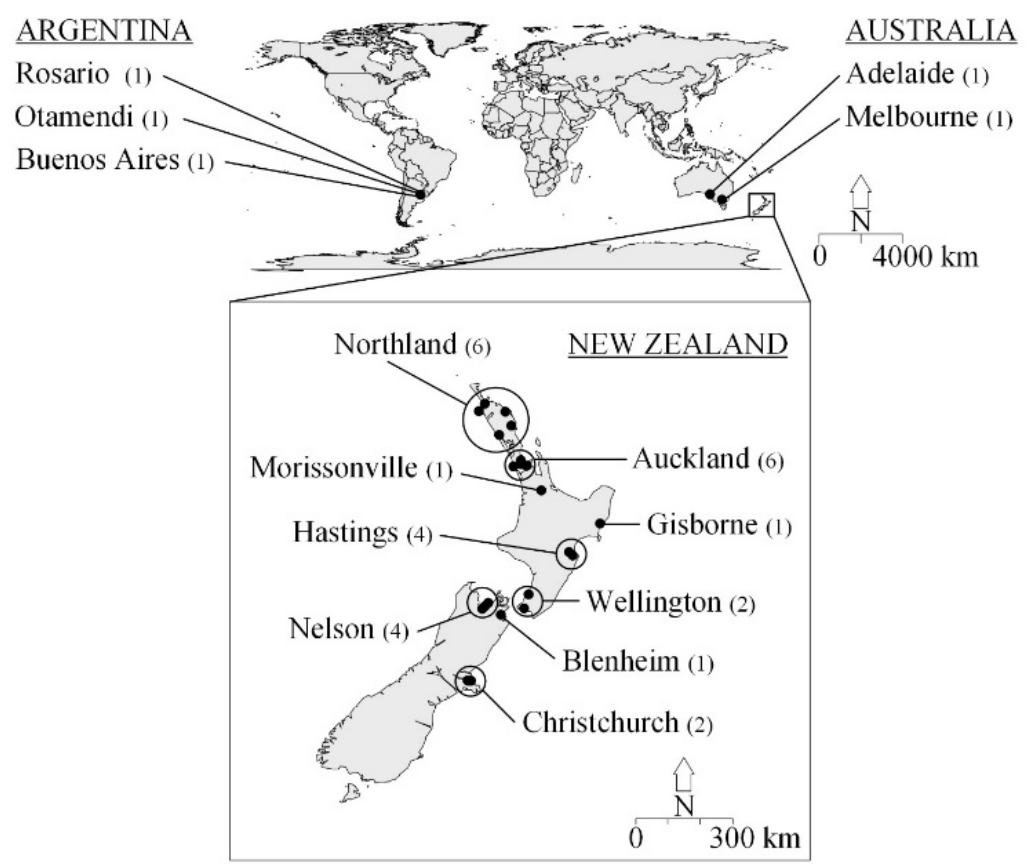

Figure 5.1: Map of the Argentine ant sampling sites. Under each sampling country name, the names of all the sampling cities/areas are listed with in the number of sites in brackets. Sampling sites consisted of: three sites in the ant home range Argentina, two in Australia the New Zealand source population, and 27 in New Zealand. 


\section{Confirmatory analysis}

RNA was extracted from a pool of 30 ants for each site using either an iPrep PureLink Virus kit (Life Technologies, Carlsbad, CA, United States), as previously described (see section 5.3.1), or a GeneJET Viral DNA \& RNA Purification Kit (Thermo Scientific, New Zealand) following the modified protocol described. Ants from each sample were ground up using pestles in micro-centrifuge tubes containing $250 \mu \mathrm{L}$ GeneJET Viral DNA \& RNA Purification Kit lysis buffer. RNA carrier $(5 \mu \mathrm{L})$ and $50 \mu \mathrm{L}$ protein $\mathrm{K}$ were added to each tube. Samples were incubated for $1 \mathrm{hr}$ in a $56^{\circ} \mathrm{C}$ heat block and mixed by pipetting at midpoint of the incubation. Samples were briefly centrifuged. Supernatant containing the lysates were transferred into a new micro-centrifuge tubes leaving behind the ant exoskeletons. The presence/absence of IAPV, KBV, APBV, DWV, SINV-1 and SINV-2, three non-coding contigs (n1000, n1905 and n1050) and five coding contigs (n6399, n6409, $\mathrm{n} 2445$, n16939 and n9293) selected from the preliminary metagenomic data (see section 5.4.1) was assessed using one step RT-PCRs using published and designed primers (Table 5.1 and 5.2). The protocol followed was as described in section 5.3.1. Primers were first tested on a pool of RNA from New Zealand sites. 
Table 5.2: Published primers used to assess the presence/absence of Israeli acute paralysis virus (IAPV), Kashmir bee virus (KBV), Acute bee paralysis virus (ABPV), Solenopsis invicta virus -1 and -2 (respectively SINV-1 and SINV-2), and Deformed wing virus (DWV).

\begin{tabular}{|c|c|c|c|c|}
\hline RNA virus & Primer name & $\begin{array}{c}\text { Primer } \\
\text { orientation }\end{array}$ & Primer sequence $\left(5^{\prime}->3^{\prime}\right)$ & $\begin{array}{c}\text { Product } \\
\text { length }(\mathrm{bp})\end{array}$ \\
\hline \multirow{2}{*}{$\begin{array}{l}\text { IAPV, KBV, ABPV } \\
\text { (Francis and Kryger, } \\
\text { 2012) }\end{array}$} & AKIF & Forward & CTTTCATGATGTGGAAACTCC & \multirow{2}{*}{$\sim 250$} \\
\hline & AKIR & Reverse & AAACTGAATAATACTGTGCGTA & \\
\hline \multirow{2}{*}{$\begin{array}{l}\text { IAPV } \\
\text { (Palacios et al., 2008) }\end{array}$} & ORF2-F8092 & Forward & CCAGCCGTGAAACATGTTCTTACC & \multirow{2}{*}{226} \\
\hline & ORF2-R8318 & Reverse & ACATAGTTGCACGCCAATACGAGAAC & \\
\hline \multirow{2}{*}{$\begin{array}{l}\text { IAPV } \\
\text { (Yang et al., 2013) }\end{array}$} & IAPV-01F & Forward & AAACATCACAGATGCTCAGGGTCGAGACTATATGT & \multirow{2}{*}{427} \\
\hline & IAPV-02R & Reverse & CTAGGGAGCTACGGAGCGTGATTCGCCTTGTAGCT & \\
\hline \multirow{2}{*}{$\begin{array}{l}\text { KBV } \\
\text { (Tentcheva et al., } \\
\text { 2004) }\end{array}$} & KBV-F & Forward & GATGAACGTCGACCTATTGA & \multirow{2}{*}{393} \\
\hline & KBV-R & Reverse & TGTGGGTTGGCTATGAGTCA & \\
\hline \multirow{2}{*}{$\begin{array}{l}\text { SINV-1 } \\
\text { (Valles and Strong, } \\
\text { 2005) }\end{array}$} & p117 & Forward & CACTCCATACAACATTTGTAATAAAGATTTAATT & \multirow{2}{*}{154} \\
\hline & p118 & Reverse & CCAATACTGAAACAACTGAGACACG & \\
\hline \multirow{2}{*}{$\begin{array}{l}\text { SINV-1 } \\
\text { (Valles and Strong, } \\
\text { 2005) }\end{array}$} & p114 & Forward & CTTGATCGGGCAGGACAAATTC & \multirow{2}{*}{647} \\
\hline & p116 & Reverse & GAACGCTGATAACCAATGAGCC & \\
\hline \multirow{2}{*}{$\begin{array}{l}\text { SINV-2 } \\
\text { (Valles and } \\
\text { Hashimoto, 2009) }\end{array}$} & p64 & Forward & ATTTGTTTGGCCACGGTCAAC & \multirow{2}{*}{318} \\
\hline & p65 & Reverse & $\begin{array}{l}\text { GATGATACAAAGCATTAGCGTAGGTAAACG } \\
\text { ATATA }\end{array}$ & \\
\hline \multirow{2}{*}{$\begin{array}{l}\text { SINV-2 } \\
\text { (Valles and } \\
\text { Hashimoto, 2009) }\end{array}$} & p548 & Forward & TGCATACTCGTTGTAAACAATCTGCTCATCT & \multirow{2}{*}{717} \\
\hline & p555 & Reverse & TGCCGTGACAATCCTGAATATCGTCAGATGTA & \\
\hline \multirow{2}{*}{$\begin{array}{l}\text { DWV } \\
\text { (Lester et al., 2015a) }\end{array}$} & DWVrtF & Forward & GCAGCTGGAATGAATGCAGAGA & \multirow{2}{*}{295} \\
\hline & DWVrtR & Reverse & ACGCGCTTAACACACGCAAA & \\
\hline
\end{tabular}

Because of mixed results and a limited amount of RNA available for each site, the complete analysis of all the contigs was not possible. Only the presence of one coding contig (n6409) and three non-coding contig (n1000, n1050 and n1905) was tested in all samples from the three different countries. The Sanger sequence quality was verified before manual editing in MEGA 6.06. Nucleotide sequences were then aligned with the contigs of interest found in the metagenomics sequencing data with ClustalW (default parameters) in MEGA 6.06 and with the best hits from BLASTn search on GenBank. Maximum likelihood trees were generated after 1,000 bootstraps of Tamura-3 parameter model with uniform rates. Outgroups were selected among the 100 best hits after BLASTn search on GenBank.

Deformed wing virus and Linepithema humile virus -1 (contig n6409) replication assessment

Both DWV and the contig n6409, tentatively named Linepithema humile virus-1 (LHUV1) (see section 5.5), are positive single strand RNA virus (de Miranda and Genersch, 2010). The detection of the negative RNA strand of the two viruses within Argentine ants is 
necessary to assess the two virus active replication within Argentine ants in Argentina, Australia and New Zealand. A pool containing an equal amount of RNA from each site was created for each country. Tagged primers (Table 5.3) were used to selectively amplify the viral negative strands during a modified one step RT-PCR as described in literature (Yue and Genersch, 2005). The 10-nucleotide long tags were generated by BARCRALW (Frank, 2009) using the default options of the program and one pair of tags was selected for each pair of primers. Tags were placed on the 5' end of the primers.

Table 5.3: Tagged primers used to amplified specifically the RNA negative strand of Deformed wing virus (DWV) and Linepithema humile virus-1 (LHUV-1) using a modified one step RT-PCR protocol. The tag sequence is written in bold lower cases.

\begin{tabular}{|c|l|l|l|c|}
\hline Virus target & \multicolumn{1}{|c|}{ Primer name } & Primer sense & Primer sequence 5' -> 3' & Product length (bp) \\
\hline \multirow{2}{*}{ DWV $^{1}$} & tag-DWVrtF & Forward & ttcgcagtaaGCAGCTGGAATGAATGCAGAGA & \multirow{2}{*}{295} \\
\cline { 2 - 4 } & tag- DWVrtR & Reverse & ccagtaactcACGCGCTTAACACACGCAAA & \\
\hline \multirow{2}{*}{ LHV-1 / n6409 } & tag-n6409-A656F & Forward & getattgagtAGGAAACGCAGCCAGTGCTATT & \multirow{2}{*}{399} \\
\cline { 2 - 4 } & tag-n6409-A1055R & Reverse & ttcttctatgGTCACCTGACTCCTTGCCTGATTT & \\
\hline
\end{tabular}

${ }^{1}$ modified from Lester et al. (2015)

A modified one step RT-PCR was undertaken using a SuperScript III One Step RT-PCR system with Platinum Taq DNA polymerase kit (Life Technologies). Reaction mixes consisted in a $24.5 \mu \mathrm{L}$ mix containing $\sim 20 \mathrm{ng}$ RNA, 1X Reaction mix (final concentration), $0.8 \mu \mathrm{M}$ of reverse tagged primer (either tag- DWVrtR or tag-n6409-A1055R - final concentration), $5.5 \mu \mathrm{L} \mathrm{ddH}_{2} \mathrm{O}$ and $1 \mu \mathrm{L}$ SuperScript ${ }^{\circledR}$ III RT/Platinum ${ }^{\circledR}$ Taq Mix. After the reverse transcription step at $45^{\circ} \mathrm{C}$ for $30 \mathrm{~min}$, the PCR machine was stopped and $0.8 \mu \mathrm{M}$ forward tagged primer (tag-DWVrtF or tag-n6409-A1055R) was added to obtain a final $25.0 \mu \mathrm{L}$ mix. The PCR thermal cycling could follow with an initial denaturation step at $94^{\circ} \mathrm{C}$ for $2 \mathrm{~min}$, followed by 35 cycles of $94^{\circ} \mathrm{C}$ for $15 \mathrm{sec}, 55^{\circ} \mathrm{C}$ for $15 \mathrm{sec}$ and $68^{\circ} \mathrm{C}$ for $30 \mathrm{sec}$, with a final extension step of $68^{\circ} \mathrm{C}$ for $5 \mathrm{~min}$. To assess the proper reverse transcription and amplification of the sequences without interference of the tags, the amplification of the positive strain of the viruses was realised in parallel. In the first step of the RT-PCR, only the tagged forward primers (either tag-DWVrtF or tag-n6409-A1055R) were added and in the second step, the tagged reverse primers tag- DWVrtR or tag-n6409A1055R) were added. After the RT-PCR cycles, RT-PCR products were visualised by gel electrophoresis on a $1.5 \%$ agarose gel stained with ethidium bromide. Amplicons were 
purified using USB ExoSAP-IT (Affymetrix) and sent for Sanger sequencing using a capillary sequencer at Massey University (Genome Service, Palmerston North, New Zealand).

\section{Deformed wing virus and Linepithema humile virus -1 (n6409) prevalence}

To assess the prevalence of DWV and LHUV-1 (n6409) infection in Argentine ant population, Argentine ant queens and workers were collected in June 2015 in New Zealand (Paraparumu, latitude 40.88336, longitude 174.99256). Ants were snap frozen and conserved at $-80^{\circ} \mathrm{C}$. RNA was extracted from 14 individual queens and one pool of 30 workers using a GeneJET Viral DNA \& RNA Purification Kit (Thermo Scientific, New Zealand) following the modified protocol previously described (see section 5.3.2). The presence/absence of DWV and LHUV-1 was confirmed following the previously described RT-PCR protocol (see section 5.3.1). To insure viral detection for the individual queen extractions, $\sim 125 \mathrm{ng}$ of RNA per sample was specifically used. No other modifications to the protocol was made. Amplicons were visualised by gel electrophoresis using a $1.5 \%$ agarose gel stained with ethidium bromide. Amplicons were purified using USB ExoSAPIT (Affymetrix) and sent for Sanger sequencing on a capillary sequencer to Massey University (Genome Service, Palmerston North, New Zealand). The quality of the Sanger sequences were checked using MEGA6 and aligned with ClustalW (using default parameters) to their original contig sequences to confirm the identity of the Sanger sequence. Because the pool of 30 ants infections by at least LHUV-1, 15 workers (collected in March 2014 from the same city and preserved in RNA later at $-20^{\circ} \mathrm{C}$ ) were used for individual RNA extraction to assess the prevalence of both DWV and LHUV-1 following the previously described protocol. After the confirmation of the identity of the amplified RT-PCR products by Sanger sequencing, the replication of the detected viruses was assessed using tagged primers (Table 5.3) and a modified one step RT-PCR, as previously described (see section 5.3.2). 


\subsection{Results}

\subsubsection{Metagenomics data analysis and confirmation of contig presence}

The Illumina sequencing data analysed in MEGAN, a total of 4,861,139 RNA and 6,251,522 DNA reads were present in the Argentine ant sample from New Zealand. After assembly in Velvet, 139,943 RNA and 8,594 DNA contigs were found. No viruses of significance were found in the DNA metagenome, and therefore DNA contigs were discarded for the rest of this study. RNA contigs best matches (i.e. 41) were viral sequences after blasting against the non-redundant nucleotide database (BLASTn) in GenBank (Figure 5.2).

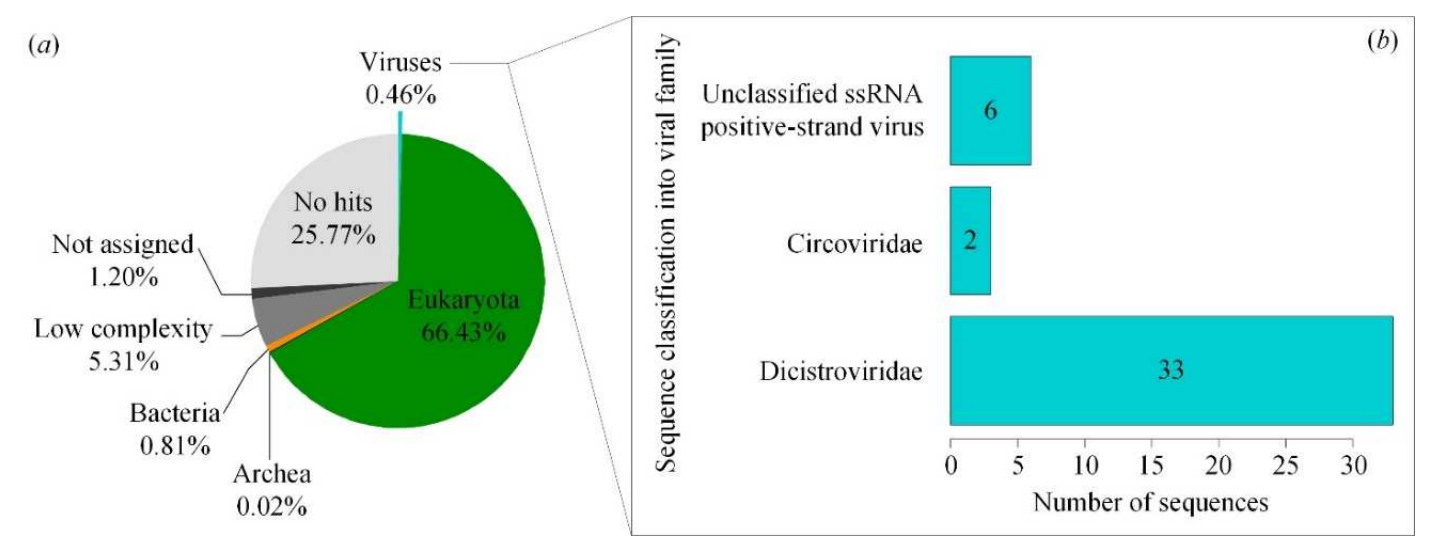

Figure 5.2: Identity of the RNA sequences found in Argentine ants: (a) Pie chart of the percentage of RNA sequences classified into each domain and viruses; (b). Classification of all the RNA sequences classified as viruses per viral family. (a) RNA sequences were classified in each domain and viruses after metagenomics analysis (complexity $=0.30$; bit score $=50)$ of RNA extraction of the Argentine ant sample from one site in New Zealand in MEGAN. (b) Sequence classification into viral family of the $0.46 \%$ of sequences classified as viruses during MEGAN analysis.

Among the 41 RNA contigs matching known viruses, 33 contigs matched viruses from the Dicistroviridae family. Among these 33 contigs, 28 contigs best hits were viruses known to infect honey bees and ants, i.e. IAPV, KBV and SINV-2. Subsequent hits for any contig may match to other related virus (see Appendix V). 
The identity of the contigs was to be confirmed using IAPV, KBV and SINV-2 published primers. The published primers for the bee viruses IAPV, KBV and ABPV, and for the ant viruses SINV-1 and SINV-2 failed to amplify any sequences within any of the pool RNA sample from each of the three countries. The use of specific primers targeting the following selected contigs, n1905 and n9293 validated the presence of the contigs in the original RNA extraction sample. The presence of the three non-coding contigs (n1000, n1905 and n1050) and five coding contigs (n6399, n6409, n2445, n16939 and n9293) were confirmed in the New Zealand RNA pool.

From the metagenomics sequencing, one coding-sequence sequence, n6409 (tentatively named Linepithema humile virus -1 - LHUV-1, see section 5.5), was selected for phylogenetic analysis. The contig was selected based on its best BLASTx match, a coding sequence for a structural polyprotein. After BLASTn search in GenBank, the best hit for n6409 was the bee virus KBV (Table 5.4). The percentage of the query identity score was low, i.e. $46 \%$. In the maximum-likelihood tree, n6409 clustered with viruses from the dicistrovirus family, the three bee viruses KBV, IAPV and ABPV, and the three ant viruses SINV-1, SINV-1A and SINV-1(TX5) (Figure 5.3).

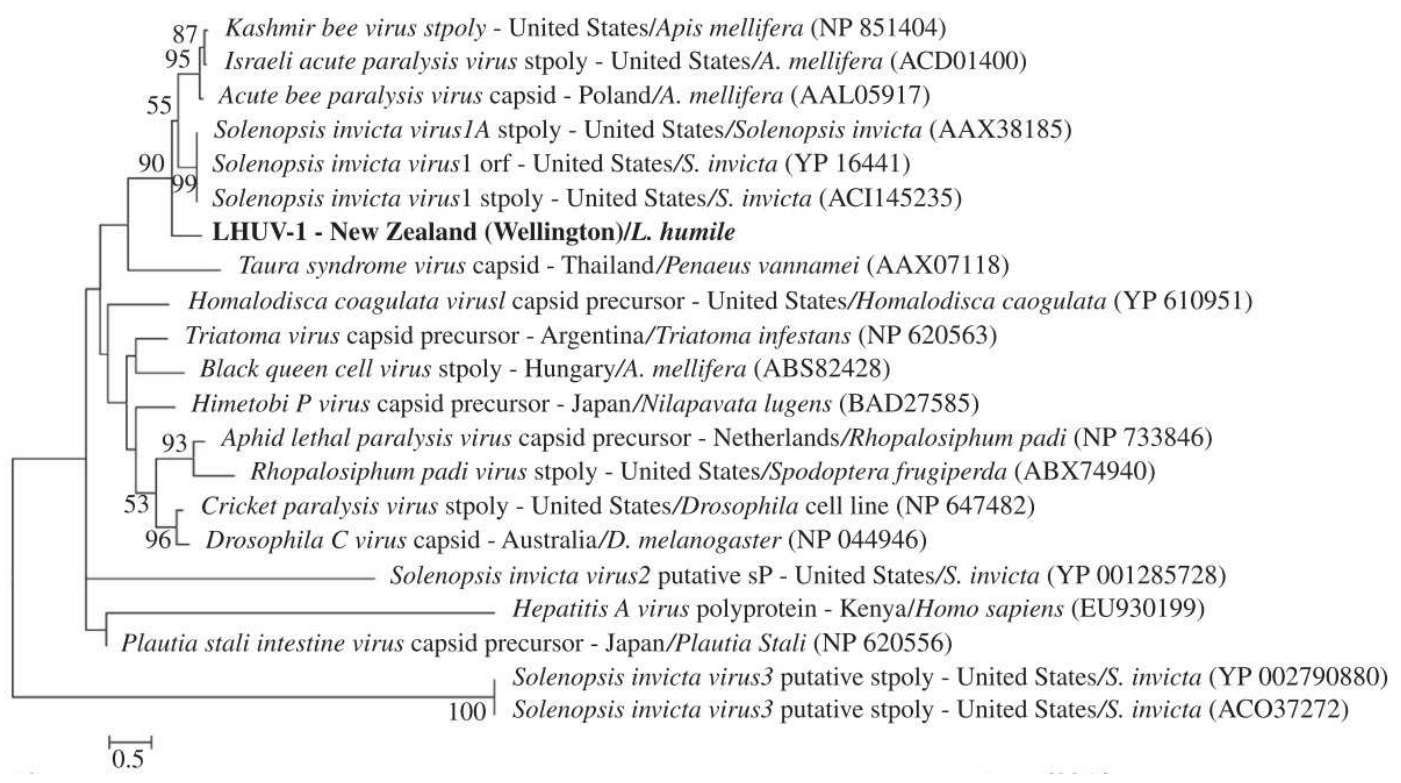

Figure 5.3: Maximum-likelihood tree of Dicistroviridae including Linepithema humile virus -1 (LHUV-1, contig n6409). The phylogenetic tree was obtained after 1,000 bootstraps using the WAG model with uniform rates in MEGA 6.06. Solenopsis invicta virus -3 was used as the outgroup. The 
sequence of interest (LHUV-1) is in bold black. Abbreviations are sP - partial structural protein and stpoly partial structural polyprotein.

\subsubsection{Distribution of Deformed wing virus, Linepithema humile virus -1 and three RNA viral sequences}

Only DWV out of the six tested viruses known to infect bees and ants, IAPV, KBV, ABPV, DWV, SINV-1 and SINV-2, known to infect honey bees and red imported fire ants, was detected by one step RT-PCR in Argentine ants using published primers. DWV was found in 22 of the 27 sites in New Zealand, but not in Argentina or Australia (Figure 5.4).

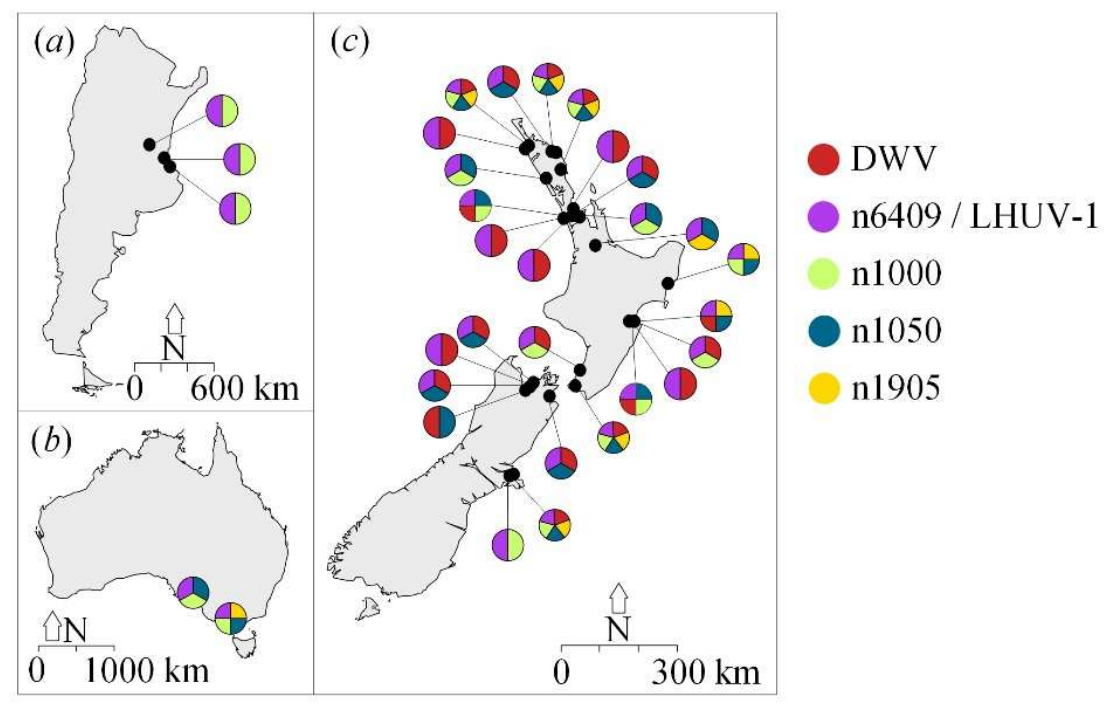

Figure 5.4: Distribution of Deformed wing virus (DWV), Linepithema humile virus -1 (LHUV-1), and three viral sequences (n1000, n1050 and n1905) in Argentine ant home range (a) Argentina and two invaded countries, (b) Australia and (c) New Zealand. Each pie correspond to one sampling site. The presence of one of the virus/contigs is indicated by the corresponding colour.

DWV sequences found in New Zealand were similar to each other but for a few bases and matched the DWV sequence found in honey bees (Apis mellifera) in New Zealand (Genbank accession AY292384 - found in New Zealand and in the United States) (Figure 5.5). 


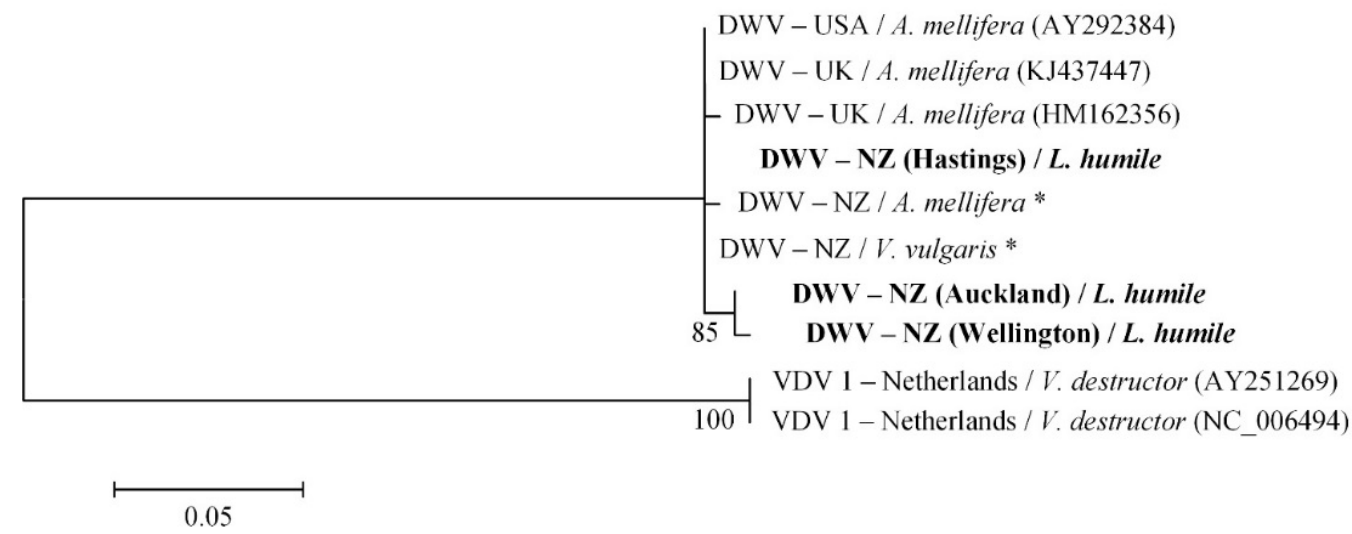

Figure 5.5: Maximum likelihood tree of Deformed wing virus (DWV) sequences from different countries. Maximum likelihood tree DWV nucleotide sequence with 1,000 bootstraps of the Tamura-3 parameter model with uniform rates in MEGA 6.06. Sequences obtained from one step RT-PCRs in this study are in bold black. Sequences with * were among the 100 best hits of DWV using a BLASTn search on GenBank. Country of origin (New Zealand - NZ, UK - United Kingdom, and the United States USA) and host organisms (Argentine ants - Linepithema humile, mites - Varroa destructor and honey bees - Apis mellifera) are indicated for each sequence.

Only the presence of the coding contig n6409 and the three non-coding contigs n1000, n1050 and n1905 was tested in each sampling site for each country. The contig n6409, a new dicistrovirus virus tentatively named Linepithema humile virus -1 (LHUV-1; see section 5.5), and the non-coding RNA sequence $\mathrm{n} 1000$, both selected from the Illumina sequencing results, were present in the three countries (Figure 5.4). LHUV-1 was detected in all sampling sites but one site, in Nelson in the South Island of New Zealand. LHUV-1 sequences from New Zealand were more closely related to the sequences from Australia (Figure 5.6). 


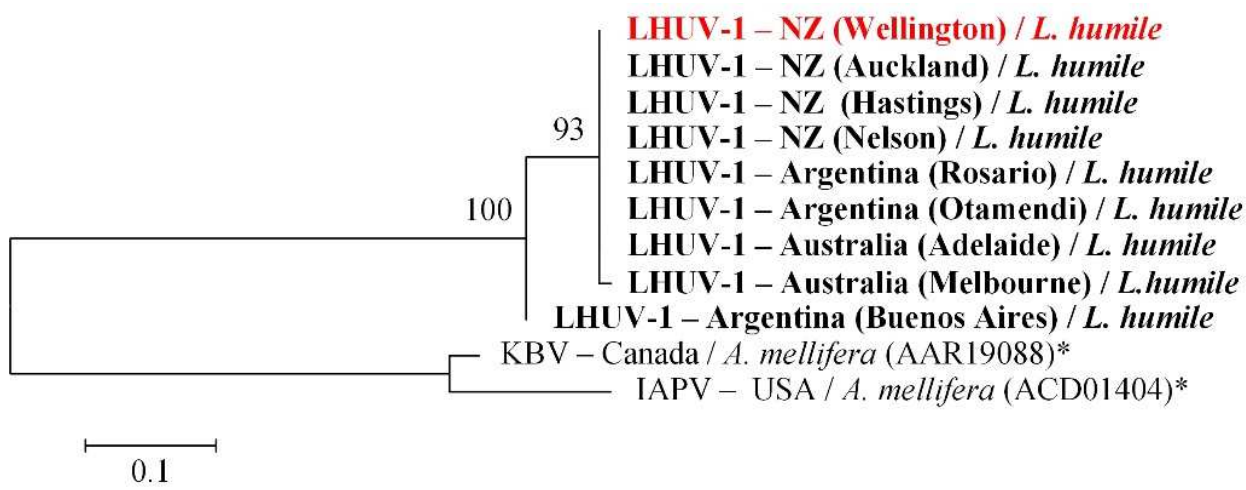

Figure 5.6: Maximum likelihood tree of Linepithema humile virus -1 (LHUV-1) sequences from different countries. Maximum likelihood tree of Linepithema humile virus -1 (LHUV-1) protein sequences with 1,000 bootstraps of the Whelan and Goldman (WAG) model with uniform rates in MEGA 6.06. The original LHUV-1 sequence (contig n6409) from the RNA metagenome analysis is in bold red. Sequences obtained from one step RT-PCRs in this study are in bold black. Sequences with * were among the 100 best hits of LHUV-1 using a BLASTx search on GenBank and corresponded to two dicistroviruses, Kashmir bee virus (KBV) and Israeli acute paralysis virus (IAPV). Country of origin (New Zealand - NZ, Canada, and the United States - USA) and host organisms (Argentine ants - Linepithema humile, and honey bees - Apis mellifera) are indicated for each sequence.

The sequence from Argentina from the Buenos Aires site was the less similar to the original LHUV-1 contig sequence from the Illumina sequencing data (Figure 5.6). The contig n1000 was present in all sites in both Argentina and Australia, while the sequence was detected in less than half the sites dispersed in New Zealand, 13 out of 27 sites (Figure 5.4). Sequences in New Zealand were more similar to the sequence present in Adelaide, Australia, than from the second Australian site, Melbourne (Figure 5.7). 


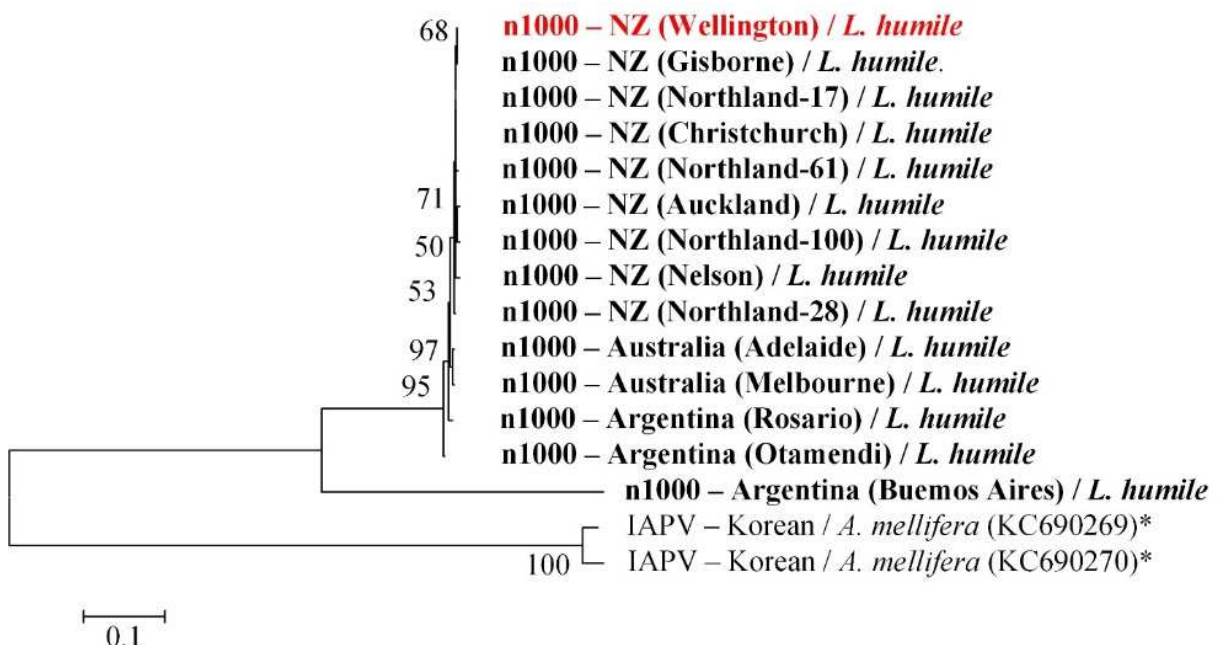

Figure 5.7: Maximum likelihood tree of contig n1000 sequences from different countries. Maximum likelihood tree of RNA metagenome contig n1000 nucleotide sequences with 1,000 bootstraps of the Tamura-3 parameter model (T92) with uniform rates in MEGA 6.06. The original RNA metagenome contig n1000 from the preliminary metagenomics analysis is in bold red. Sequences obtained from one step RT-PCRs in this study are in bold black. Sequences with * were among the 100 best hits of contig n1000 using a BLASTx search of GenBank and corresponded to the dicistrovirus Israeli acute paralysis virus (IAPV). Country of origin (New Zealand - NZ, Australia, and Korea) and host organisms (Argentine ants - Linepithema humile, and honey bees - Apis mellifera) are indicated for each sequence.

The other two tested non-coding sequences selected from the Illumina sequencing results (i.e. n1905 and n1050) were only shared between Australia and New Zealand. The contig n1050 was found in both sites in Australia and in 18 sites in New Zealand (Figure 5.4). The sequence from New Zealand were more similar to the sequence from Melbourne, the historical population of origin of New Zealand population, than to the sequence from Adelaide (Figure 5.8). 


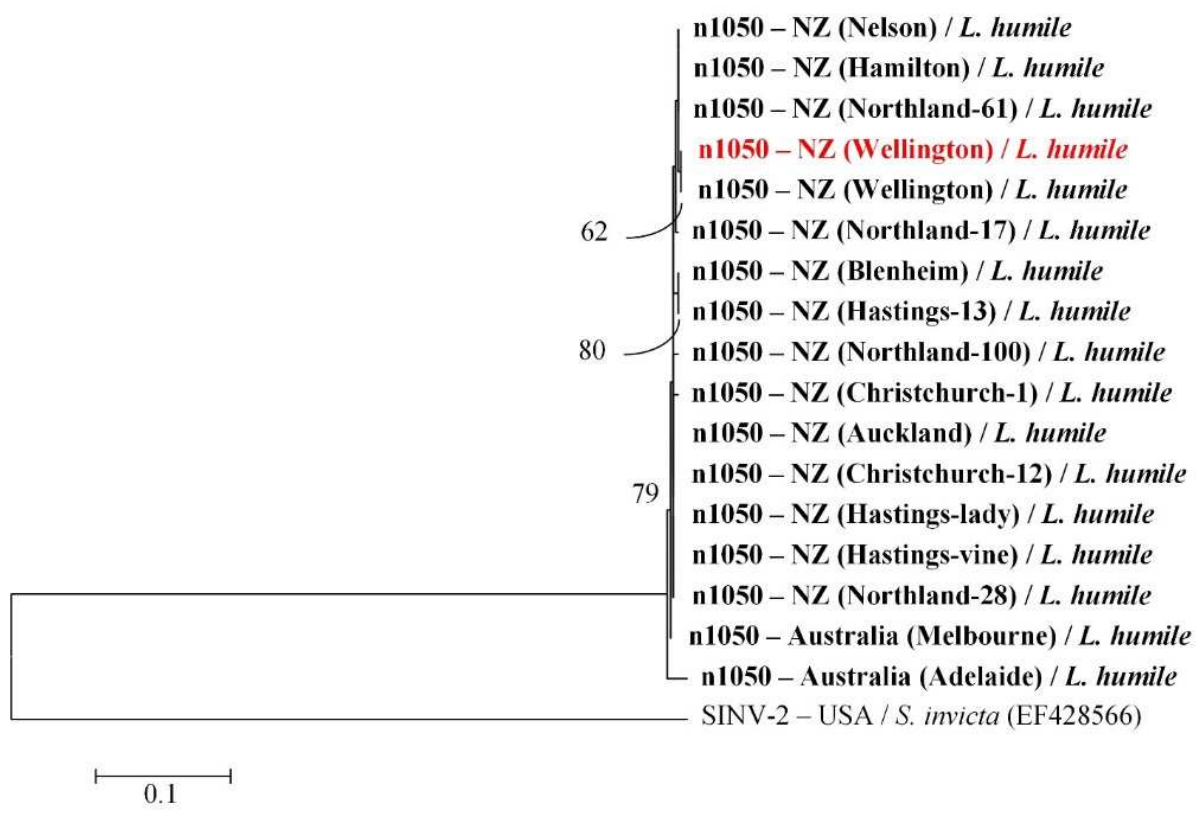

Figure 5.8: Maximum likelihood tree of contig n1050 sequences from different countries. Maximum likelihood tree of RNA metagenome contig n1050 nucleotide sequences with 1,000 bootstraps of the Tamura-3 parameter model with uniform rates (T92) in MEGA 6.06. The original RNA metagenome contig n1050 from the preliminary metagenomics analysis is in bold red. Sequences obtained from one step RT-PCRs in this study are in bold black. Sequence with * was among the 100 best hits of contig n1050 from BLASTx searches on GenBank, and corresponded to the dicistrovirus Solenopsis invicta virus-2 (SINV-2). Country of origin (New Zealand - NZ, Australia, and United States - USA) and host organisms (Argentine ants - Linepithema humile, and red imported fire ants - Solenopsis invicta) are indicated for each sequence.

The contig n1905 was found in Melbourne, one of the two sites in Australia, as well as in 8 sites in New Zealand (Figure 5.4). Again, the sequence from New Zealand were more similar to the sequence from Melbourne, the historical population of origin of New Zealand population, than to the sequence from Adelaide (Figure 5.9). 


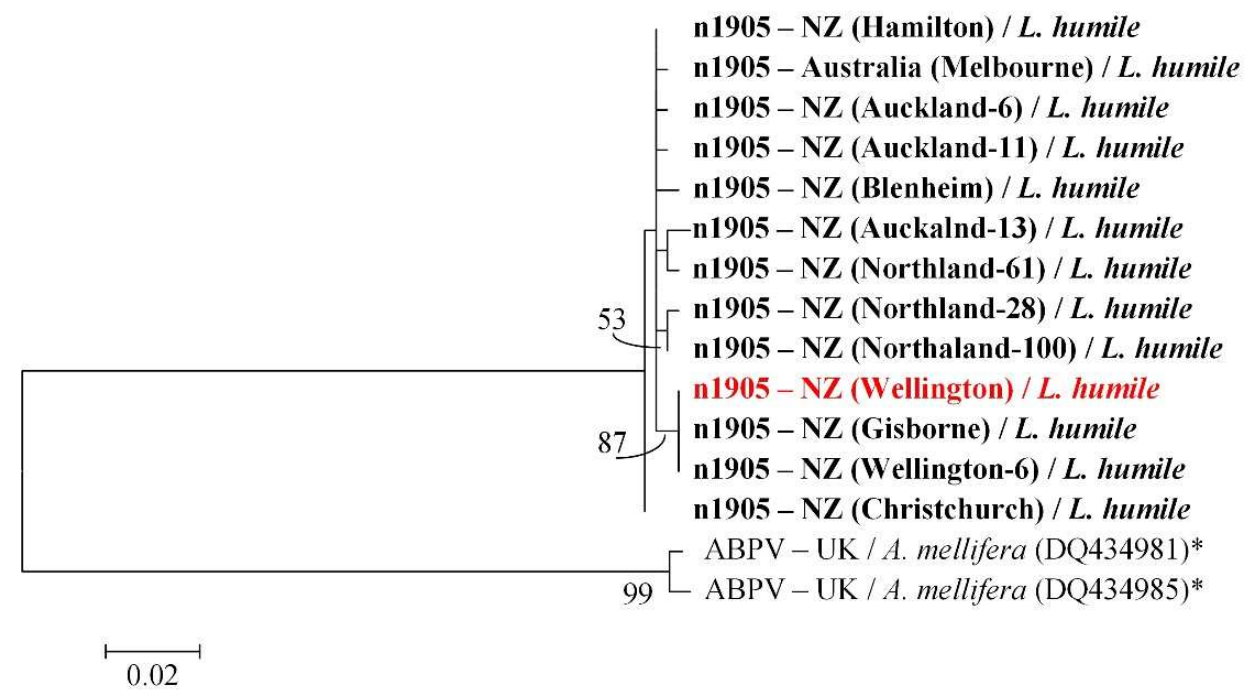

Figure 5.9: Maximum likelihood tree of contig n1905 sequences from different countries. Maximum likelihood tree of RNA metagenome contig n1905 nucleotide sequences with 1,000 bootstraps of the Tamura-3 parameter model with uniform rates (T92) in MEGA 6.06. The original RNA metagenome contig n1905 from the preliminary metagenomics analysis is in bold red. Sequences obtained from one step RT-PCRs in this study are in bold black. Sequences with * were among the best hits of contig n1905 from BLASTx searches on GenBank and corresponded to the dicistrovirus Acute bee paralysis virus (ABPV). Country of origin (New Zealand - NZ, United Kingdom- UK) and host organisms (Argentine ants - Linepithema humile, and honey bees - Apis mellifera) are indicated for each sequence.

\subsubsection{Active replication and prevalence of Deformed wing virus and Linepithema humile virus $\mathbf{- 1}$}

The negative strands of both DWV and LHUV-1 were amplified in Argentine ants using the modified one-step RT-PCRs and only the tagged reverse primers were used during the reverse transcription stage. The presence of the negative strands indicates an active replication of the viruses within the ants. DWV positive and negative strands were amplified in Argentine ant pool sample from New Zealand. No DWV amplification of either the positive or the negative strands was detected in the sample pool from Argentina. LHUV-1 positive and negative strands were amplified in the sample pool from each of the three countries of interest, Argentina, Australia and New Zealand.

DWV was found in only one worker $(n=15)$ while LHUV-1 was found in one queen $(\mathrm{n}=14)$ and two workers $(\mathrm{n}=15)$. No co-infections were detected. Both viruses appeared to be actively replicating within the infected individuals. 


\subsection{Discussion}

To our knowledge, this is the first study to report the presence of any viruses in the invasive Argentine ant. The bee virus Deformed wing virus (DWV) and 41 RNA potential viral sequences were discovered in Argentine ants from New Zealand. Three RNA viral noncoding sequences (i.e. n1000, n1841 and n1905), and one coding sequence (n6409) were found to be closely related to two harmful bee viruses, Israeli acute paralysis virus (IAPV) and Kashmir bee virus (KBV). The coding n6409 sequence was tentatively named Linepithema humile virus -1 (LHUV-1). Furthermore, DWV and LHUV-1 actively replicated within the ants. The Argentine ant that I sampled did not present any infection by the bee viruses IAPV, KBV and Acute bee paralysis virus (ABPV), and the red imported fire ant (Solenopsis invicta) viruses Solenopsis invicta virus -1 and -2 (respectively SINV1 and SINV-2) (Table 5.4).

Table 5.4: Summary table of the presence/absence of viruses of interest in Argentina, Australia and New Zealand. The six "known viruses" are four viruses involved in honey bee colony collapse disorder (Israeli acute bee paralysis - IAPV, Kashmir bee virus - KBV, Acute bee paralysis virus $\mathrm{ABPV}$, and Deformed wing virus - DWV) and two viruses which can lead to the death of entire red imported fire ant colonies (Solenopsis invicta virus -1 and -2 - respectively SINV-1 and SINV-2). Data was compiled from literature reports in diverse arthropods such as honey bees and bumblebees. The four novel RNA sequences are Linepithema humile virus -1 (LHUV-1) and three contigs (n1000, n1050 and n1905) found in and found in this study in Argentine ants (Arg. ants) metagenomics data. Presence $\checkmark /$ absence $x$ symbols are both in red for one country when published data and newly discovered data in Argentine ants matched. The symbol ? was used when no published data was available.

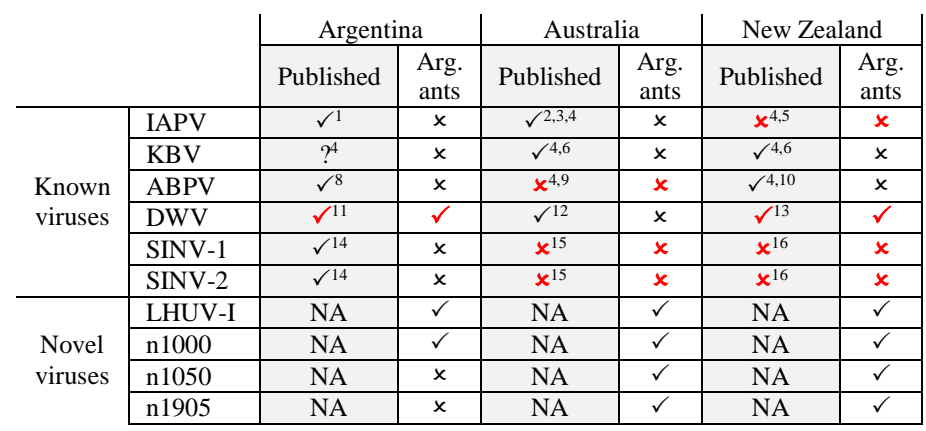

${ }^{1}$ (Reynaldi et al., 2011)

${ }^{2}$ (Cox-Foster et al., 2007)

${ }^{3}$ (Palacios et al., 2008)

${ }^{4}$ (de Miranda et al., 2010)

5 (McFadden et al., 2014)

${ }^{6}$ (DALL, 1985)
${ }^{7}$ (Todd et al., 2007)

${ }^{8}$ (Reynaldi et al., 2010)

${ }^{9}$ (de Miranda et al., 2010)

${ }^{10}$ (Todd et al., 2007)

${ }^{11}$ (Reynaldi et al., 2013)
${ }^{12}$ (Singh et al., 2010)

${ }^{13}$ (Todd et al., 2007)

${ }^{14}$ (Valles et al., 2009)

${ }^{15}$ (Yang et al., 2010)

${ }^{16}$ (Yang et al., 2010) 


\section{First detection of viruses in Argentine ants}

One bee virus, DWV, and 41 potential viral sequences were detected in Argentine ants in the original sample from New Zealand. The detection of viruses and co-infections in Argentine ants was anticipated given that viruses have been observed in other ant species. Six viruses have been described for biological control purposes in the red imported fire ant, including SINV -1, -2 and -3 (Valles et al., 2004, 2007a, 2007b, 2013b; Valles and Strong, 2005; Hashimoto and Valles, 2008; Valles and Hashimoto, 2009; Porter et al., 2013). A new totivirus, Camponotus yamaokai virus, was recently described in the arboral ant $(C$. yamaokai) (Sassa et al., 2015) as well as viral sequences in the Caribbean crazy ants (Nylanderia pubens) (Valles et al., 2012; Valles and Oi, 2014) and in the narrow headed ants (Formica exsecta) (Johansson et al., 2013). Even a bee virus, the Chronic bee paralysis virus (CBPV), was reported in the ants C. vagus and Formica rubra (Celle et al., 2008).

\section{New viral sequences including Linepithema humile virus -1 found in Argentine ants}

The sequence n6409 from Argentine ants clustered with coding sequences of Discistroviridae viruses, a family of viruses that included multiple viruses such as IAPV, $\mathrm{KBV}$ and ABPV involved in colony collapse disorder in honey bees (Apis mellifera) (Figure 5.3). The sequence identity with its best match on BLASTn (IAPV) was low. In addition, the use of specific IAPV primers did not amplify any sequences in the tested sample where n6409 was discovered. These results indicated that n6409 may be part of the genome of a new dicistrovirus RNA positive strand virus. This new virus was tentatively named Linepithema humile virus -1 (LHUV-1). Further analyses and genome sequencing of LHUV-1 are necessary to ensure its new viral identity.

LHUV-1 was found to actively replicate within Argentine ants. The ants may therefore constitute a potential reservoir for LHUV-1: the virus may replicate and multiply within the ants before potentially spreading to other susceptible species. The specificity and the effects of LHUV-1 are as yet unknown. The large range of the virus may inform on its potential effects. The presence of the virus in this successful invasive species both in the ant home range, Argentina, and two invaded countries, Australia and New Zealand, suggests two hypotheses: 1) a beneficial interaction between the ants and the virus, or 2) a latent infection of the virus. Not all viruses have detrimental effects on their hosts (Roossinck, 2011; Xu et al., 2014). For example, the symbiosis involving polydnaviruses 
and parasitoid wasps promotes the fitness of the viruses and their wasps hosts at the detriment of a third host parasite by the wasps (Strand and Burke, 2013). If the association between the ants and the virus was beneficial for both parties, infected ants may have been favoured during the invasion process compared to uninfected ants and infected population may be larger than uninfected ones. LHUV-1 may promote Argentine ant fitness and facilitate the spread of the ants. However, LHUV-1 may more likely be detrimental to the ants. LHUV-1 seems to be a member of the Dicistroviridae family, a family of viruses that includes viruses involved in the honey bee colony collapse disorder, such as IAPV, KBV and ABPV (Bonning and Miller, 2010). Infections by IAPV, KBV and ABPV weaken infected bee hives, and can lead to the death of infected colonies (de Miranda et al., 2010; Chen et al., 2014). Aside from their negative effects on honey bee populations, IAPV and $\mathrm{KBV}$ infections were shown to slow offspring production of bumblebees (Bombus terrestris) under laboratory conditions (Meeus et al., 2014). These viruses can be present inside colonies at low prevalence without presenting symptoms (de Miranda et al., 2010; Chen et al., 2014). The intervention of mites (Varroa destructor) in the infection process increases the virulence of the infections (de Miranda et al., 2010). LHUV-1 pathogenicity may be similar to IAPV, KBV and ABPV: infections are present in low proportion in the colonies in a latent state ants, and the virulence of the virus may then be triggered by an external factor (i.e. infection by another micro-organism or stress). As the virus is present in most of the sites in New Zealand, the virus is a potential candidate to explain the decline of some parts of Argentine ant population in New Zealand (Cooling et al., 2012).

The other sequences, n1000, 1050 and n1905, clustered with non-coding viral RNA sequences. Therefore their definitive viral identity cannot be determined with certainty (respectively Figures 5.7, 5.8 and 5.9). The absence of specific co-occurrences / coinfections in the sequence distributions in Argentine ants in the different sites suggests that LHUV-1 and the three non-coding RNA sequences are part of four distinct viruses. Further sequencing analyses may offer larger sequences of n1000, 1050 and n1905 and inform on their identity with high confidence.

\section{Detection of Deformed wing virus but no other bee nor ant viruses}

In addition to LHUV-1 and the three non-coding sequences, the bee virus DWV was detected in Argentine ants in Argentina and New Zealand (Figure 5.4). The presence of 
DWV in Argentine ants from Argentina and New Zealand concords with the known presence of the virus in both countries (Table 5.4). In contrast, even though DWV is present in Australian honey bees, the virus was not detected in Argentine ants. Further sampling in Australia is necessary to ensure the absence of DWV in Argentine ants, only two sites were analysed in this study.

The low prevalence of DWV in Argentine ants may be due to the sampling season (i.e. winter). DWV infection rate fluctuates during the year in honey bees, and is less prevalent during cold seasons (Dar and Kotwal, 2014). Further sampling is therefore necessary to assess the prevalence of the virus in Argentine ant colonies.

DWV sequence found in Argentine ants from Argentina and New Zealand differed from each other (Figure 5.5). The DWV sequence in New Zealand Argentine ants is similar to the sequence of found in honey bees in New Zealand. Therefore DWV infections in Argentine ants in New Zealand may have occurred by horizontal transfer either by direct contact with infected individuals or from the ant new environment. Inter-species infections by DWV have been reported via contact with contaminated pollen (Singh et al., 2010). Argentine ants may have been infected by DWV while foraging on contaminated pollen. The ants may also have been infected during a foraging raid on an infected bee hive, via contact with contaminated wax, ingestion of contaminated honey and bee brood (Eyer et al., 2009).

Active replication of DWV was detected in Argentine ants. This result concords with previous reports indicating that DWV actively replicates within multiple arthropod orders (Levitt et al., 2013). Replication was only detected in ants from New Zealand. The absence of observed replication in ants from Argentina may be due to the different DWV strain present in the ant home range (no DWV sequences from Argentina were found on GenBank). The primers were originally designed to amplify the DWV strain found in New Zealand (Lester et al., 2015a). Consequently, the primers may have a lower specificity with the strain present in Argentina. This low specificity was further decreased by the addition of the 10-nucleotide tags on the 5' end of the primers. The hypothesis of low specificity of the tagged primers was supported by the absence of amplification of the DWV positive strand by the tagged primers while DWV was amplified by non-tagged primers. DWV may therefore actively replicate within ants in Argentina. Different tagged primers are necessary to further investigate the question. Overall, the active replication of DWV in ants in New 
Zealand indicates the possible reservoir role of the ants in the spread of bee viruses by spillback.

The effects of the virus on Argentine ants is unknown. The virus is present in multiple ant sites as identified in this study. If pathogenic, it is probable that DWV has a low virulence in Argentine ants. DWV virulence in honey bees is low except when horizontally transmitted by mites (V. destructor) (de Miranda et al., 2010; Ryabov et al., 2014). Similarly, DWV virulence may be triggered in Argentine ants after the intervention of a third party, such as another micro-organism or abiotic factors. It is also possible that DWV only affects the ants throughout the winter season. DWV has indeed been reported to cause losses of bee hives over wintering bee hives (Highfield et al., 2009). Additional comparative studies on fitness of DWV infected and non-infected Argentine ant colonies may inform on the pathogenicity and virulence of DWV in the ants.

The absence of infections by IAPV, ABPV and KBV, three other viruses involved in CCD, and SINV-1 and SINV-2, two ant viruses, in Argentine ants differs from the reported viral landscape of each country (Table 5.4). The only exceptions were for: IAPV and ABPV which are already reported as absent respectively in New Zealand and in Australia, and SINV-1 and SINV-2 never reported in Australia and New Zealand (Table 5.4). Three hypotheses may explain the absence of observed infections in ants: 1) IAPV, KBV and ABPV as well as SINV-1 and SINV-2 cannot infect Argentine ants; 2) low prevalence during the sampling season; 3) infected ants were not sampled. Firstly, SINV-1 and SINV2 were previously reported to be specific to fire ant species (Valles et al., 2007b). However, non-infection capacity of Argentine ants by IAPV, KBV and ABPV is unlikely as these three viruses have been previously detected in other arthropods species (Singh et al., 2010; Levitt et al., 2013). Secondly, the absence of the viruses in Argentine ants may otherwise be due to the sampling season. Some virus infections present seasonal variations and may be more difficult to detect during specific periods (Valles et al., 2007b, 2010; Runckel et al., 2011). However, this hypothesis is unlikely as bee virus infections are persistent in bees, and can be detected both in summer and winter seasons (Tentcheva et al., 2004; Chen et al., 2014). Thirdly, none of the collected ants may have come from colonies that had direct or indirect contacts with any infected bees. The low number of sites studied in Argentina and Australia lowers the probability of detecting infections rates of IAPV, ABPV and $\mathrm{KBV}$ in the ants. In contrast, the high number of sampling sites in New Zealand covering most of the Argentine ants' range in the country indicates that the viruses are 
effectively absent in the ants at least in New Zealand. This hypothesis is supported by the reported absence of IAPV and the low ABPV and KBV infection rate in New Zealand bee hives (Todd et al., 2007; de Miranda et al., 2010; McFadden et al., 2014; Mondet et al., 2014).

The presence in Argentine ants of one virus (i.e. DWV) involved in colony collapse disorder in most of the ant range in New Zealand may be a first insight in explaining the reported Argentine ant population decline in the country (Cooling et al., 2012). Viruses have in fact been associated with population decline (Porter et al., 2013) and are studied as possible bio-control agents. Further experimentations are needed to better understand the effects of DWV on Argentine ant fitness.

\section{Virus invasion pathway}

I suggest multiple infection scenarios (Figure 5.10) based on the incidence of the new virus LHUV-1, the three investigated viral contigs (n1000, n1841 and n1905) and the honey bee virus DWV in Argentine ant samples (Figure 5.5). 


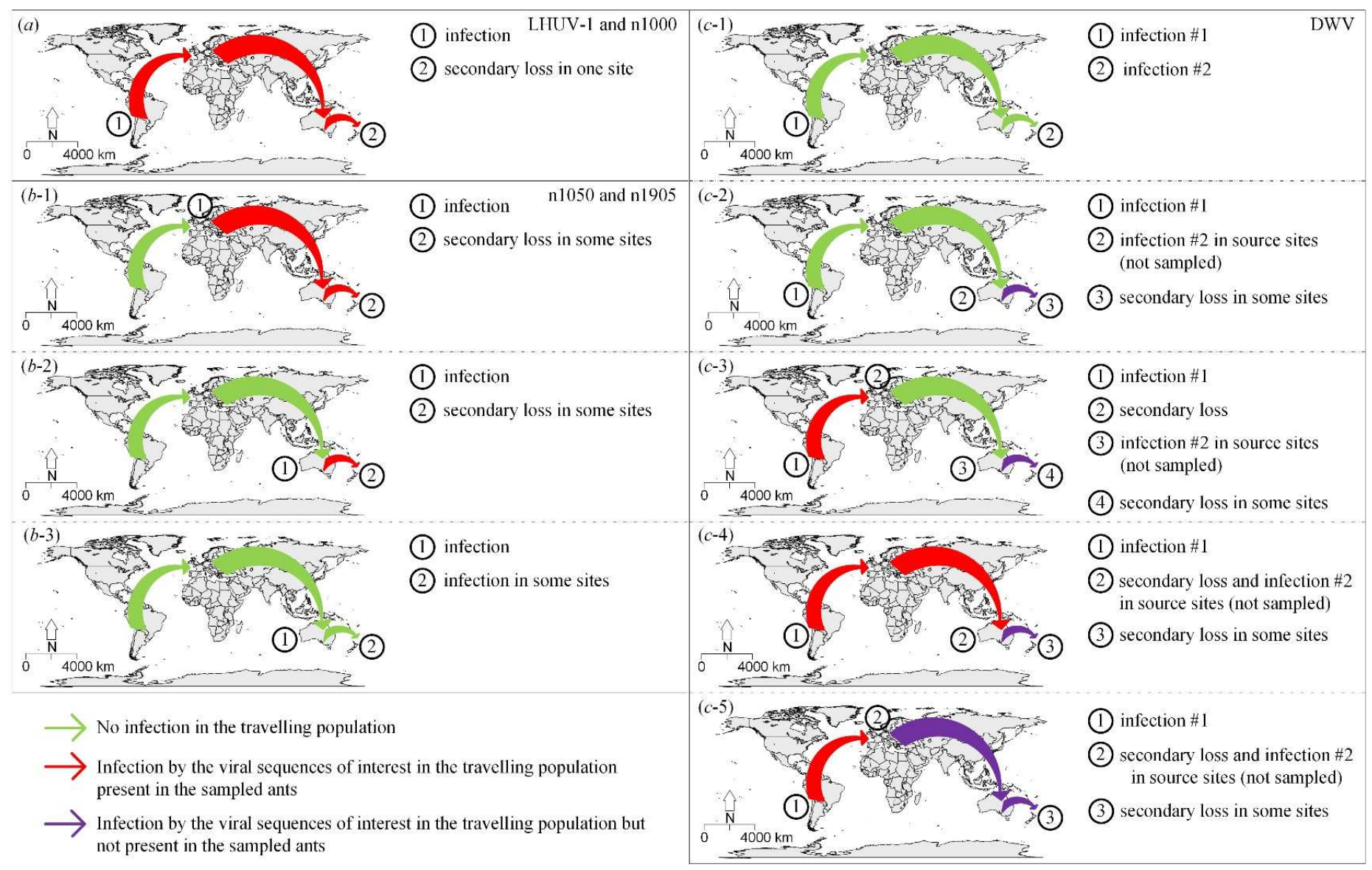

Figure 5.10: Infection scenarios occurring at different stage of Argentine ant invasion path for: (a) Linepithema humile virus -1 (LHUV1) and n1000; (b -1 to -3) n1050 and n1905; and (c -1 to -5) Deformed wing virus (DWV). Each scenario are probable infection events along the invasion path of Argentine ants, from their home range in South America to Europe, then from Europe to Australia and finally from Australia to New Zealand. The different arrow colours indicate the infection state of the travelling ant populations. Possible events are indicated by circle numbers in historical order and located on the map next to their geographical occurrence. 
Diverse scenarios involve potential infections and occurrence of secondary losses while the Argentine ant population was transported from Argentina to Europe, Australia and finally New Zealand (Corin et al., 2007a, 2007b; Suhr et al., 2011). Viral sequence mutations may have occurred during the multiple ant population movements and bottlenecks. The mutations may have led to an increase in the differences between sequences from different countries as it was observed with LHUV-1 (Figure 5.7). In the case of DWV, relatively high difference between sequences (Figure 5.6) indicates two different viral strains present in the ants. Therefore, the most probable infection scenarios involve at least two independent events of DWV infections. From the distribution of the different viruses and the sequence differences, the most probable infection scenarios are: LHUV-1 and n1000 infections originated from Argentina (Figure 5.11.a); n1050 and n1905 infections originated from Australia or Europe (Figure 5.11.b-2 and -2); DWV infections occurred in New Zealand during the Argentine ant range expansion within the country (Figure 5.11.c1). If LHUV-1 and n1000 were co-introduced with Argentine ants in New Zealand, this cointroduction would concords with other examples of invasive species co-introducing pathogens from their home range (Strauss et al., 2012). The co-introduction of pathogens can be at the detriment of native species if the viruses cross species barriers. The low number of sampling sites in Argentina and in Australia as well as the absence of samples from Europe limits the selection of a lower number of hypothesis on the origins of the different viruses. DWV infections may have indeed originated from Argentina and no samples with a DWV strain similar to the New Zealand one were collected in this study. Furthermore, DWV RNA may have degraded in the Australia samples as the ants were preserved at $4^{\circ} \mathrm{C}$ in ethanol while the other samples were conserved at $-20^{\circ} \mathrm{C}$ and $-80^{\circ} \mathrm{C}$. The sequences n1050 and n1905 may also be present in Argentina but no infected samples were collected. Further work examining these sequences and potential viruses in their home and invaded range is necessary.

\section{Critical evaluation of experimental procedures}

If additional sampling had been possible, increasing the number of sampling sites in Argentina and Australia would have offered a stronger support to the discovered range of the viruses. Further sampling in Europe would also allow to the selection of fewer infection 
scenarios, and increase knowledge on the range of the viruses found in Argentine ants. Sampling workers and queens during different seasons would offer an insight on virus prevalence and any possible seasonal variations. Funding allowed the investigation of multiple known viruses and new sequences in Argentine ants. Not all viral sequences were investigated. The development of additional specific primers to amplify viral sequences matching coding sequences (contigs mqtching coding sequences after BLASTn search on GenBank) may allow the discovery of additional new viruses in Argentine ants.

Argentine ants are the hosts to multiple viruses including the bee virus DWV in Argentina and New Zealand, and the new dicistrovirus LHUV-1 in Argentina, Australia and New Zealand. Both viruses replicates within the ants. No infections by the bee viruses IAPV, $\mathrm{KBV}$ and $\mathrm{ABPV}$, and by the red imported fire ant viruses SINV-1 and SINV-2 were detected. LHUV-1 may have been co-introduced by the ants from their home range. DWV infections may have occurred in New Zealand during the Argentine ant range expansion in the country. These results suggest a co-introduction of viruses with the Argentine ant population in New Zealand and a potential reservoir role of the ants. The large range of Argentine ants in the world and more specifically in New Zealand may promote the spread of harmful viruses possibly affecting native species. Both DWV and LHUV-1 are candidates to explain the population decline of Argentine ants. Further study on the specific effects of the viruses on the ants are necessary. This case study highlight the unseen viral threat that invasive species can represent to native species. 
Chapter 6. General discussion 


\subsection{Presence of bacteria and viruses in Argentine ants}

This thesis was the first examination of bacteria and viruses present in Argentine ants. Bacteria decreased along the ant invasion pathway while a core of bacteria was maintained (see Chapter 2). The high abundance of bacteria from Lactobacillus and Gluconobacter genera among the ant core bacteria indicates that these bacteria may have a beneficial relationship with Argentine ants and may play a major role in the ant health and fitness. Antibiotic treatments with ampicillin and gentamicin did not eliminate these two bacteria from the ants (see Chapter 3). Therefore, no information on the role of these two bacterial groups in the ants could be gathered. Nevertheless, antibiotic treatments increased bacterial diversity and Argentine ant survival. Antibiotic treatments with ampicillin, kanamycin and spectinomycin did not induce inter-specific aggression (see Chapter 4). Spectinomycin treatments increased Argentine ant intra-species aggressiveness. Bacterial diversity may therefore be one factor influencing Argentine ant survival and aggressive behaviour, and at length Argentine ant population dynamics. The absence of any effects on fitness may be caused by the experimental design where the queens were not being tested. In addition, potentially harmful viruses were discovered in Argentine ants: Deformed wing virus (DWV), a honey bee virus involved in colony collapse disorder, and a new dicistrovirus, Linepithema humile virus -1 (LHUV-1) (see Chapter 5). These two viruses are potential candidates to explain the Argentine ant population decline previously reported in New Zealand (Cooling et al., 2012).

\subsection{The decrease of bacterial diversity in invasive Argentine ants was associated with a complex invasive pathway and low genetic diversity}

Invasive species typically present a lower genetic diversity in their invasive range than in their home range (Puillandre et al., 2008). Founding events and propagule pressure may cause genetic bottlenecks, leading at length to the loss of rare alleles and a low genetic diversity in invasive species (Dlugosh and Parker, 2008). Argentine ants were introduced from South America to Europe (primary invasion), from Europe to Australia (secondary invasion) and from Australia to New Zealand (tertiary invasion), in what was likely a single invasive event (Corin et al., 2007b; Wetterer et al., 2009). This complex invasion pathway 
lead to multiple consecutive founding events and genetic bottlenecks. Genetic diversity in the invasive ants was reported to be lower in its invasive range than in its home range (Corin et al., 2007a; Brandt et al., 2009). However, it seems that founding events also affected bacterial diversity present in these invasive ants (see Chapter 2). Bacteria diversity decreased along the ant invasion pathway and was the lowest in New Zealand, a country invaded after a tertiary invasion. Interestingly, in the United States, where the Argentine ant population originated from multiple primary invasions, presented a bacterial diversity similar to the one in the ant home range. Invasion history and pathway seem then to affect bacterial diversity present in invasive species.

Even though bacterial diversity declines along the invasion pathway, invasive species seem to conserve a core of nine bacterial genera. Argentine ants in all countries and all sites maintained a core of bacteria that included Lactobacillus and Gluconobacter. Both Lactobacillus and Gluconobacter have reported to include beneficial bacteria species, beneficial in bees (Apis spp.) for example (Kaltenpoth and Engl, 2014). Bacteria from these genera seem involved in their host health and defence against some pathogenic infections. However, the full effects of the presence of these bacteria on Argentine ants is yet unknown. It could be postulate that associations with beneficial bacteria from genera such as Lactobacillus and Gluconobacter may promote invasive species population growth and spread.

\subsection{Potential role of bacterial diversity in Argentine ant population dynamics}

High genetic and bacterial diversity are associated with individual health (Mattila et al., 2012). For example, genetically diverse colonies of the ants Formica selysi were more resistant to pathogenic fungal infections than less diverse colonies (Reber et al., 2008). In bees, genetically diverse individuals presented higher bacterial diversity and improved health than individuals with lower genetic diversity (Mattila et al., 2012). Antibiotic treatments of Argentine ants did increase bacteria diversity present in the ants and increased the ant survival (see Chapter 3 and 4). The low genetic and bacterial diversity of Argentine ants may lower their immune defence against pathogenic infections. The low genetic and 
bacterial diversity of Argentine ants in New Zealand may be two of the factors involved in the observed population decline (Cooling et al., 2012).

In addition, invasive species population dynamics may be affected by the intra- and interspecific aggressiveness. A lack of invasive species aggression is one of the mechanisms thought to be involved in the success of invasive species in their new range (Fadmiro et al., 2009; Lai et al., 2015). With aggressive behaviour (i.e. biting, pulling, killing), species may be more capable of defending their territory and food sources. For example, Argentine ants monopolise food sources against other ant species with a high number of workers and high aggression levels (Human and Gordon, 1996).

The lack of intra-specific aggression in Argentine ants appear to promote cooperation between nests and the recruitment of a high number of workers (Suarez et al., 1999; Tsutsui et al., 2000). If intra-specific aggression was to occur in Argentine ants, they may allocate fewer workers to find or defend food sources while spending more energy defending their nest against other Argentine ants. At length, colony fitness would then be lowered. Nevertheless, the change of bacterial diversity induced by antibiotic treatments did not modify intra-species aggressiveness in Argentine ants. Therefore bacterial diversity in Argentine ants may not be involved in nestmates recognition, as it is the case for termites (Matsuura, 2001). The bacterial diversity present in the ants may affect the ant health and therefore modify outcomes as a result of inter-species encounters. Treatment with one antibiotic (i.e. spectinomycin) did increase the ant inter-species aggressiveness (see Chapter 4). Further analyses are need to assess if the antibiotic treatment induced an increase or a decrease in bacterial diversity. In natural conditions, Argentine ants may suffer changes in their bacterial diversity during the invasion process or the change in food sources. These bacterial diversity modification may be involved in the ant intra-specific aggression level and thus eventually may affect the ant competitiveness success.

\subsection{Potential role of viral infections in Argentine ant population dynamics}

Population declines may be caused by pathogenic infections, including viral infections (Evans and Schwarz, 2011; Strauss et al., 2012). In the invasive red imported fire ants (Solenopis invicta), virulent infections by Solenopsis invicta virus $-1,-2$ and -3 
(respectively SINV-1, SINV-2, and SINV-3) may cause the death of entire colonies (Hashimoto and Valles, 2008; Oi and Valles, 2009; Valles and Porter, 2015). Multiple viruses are involved in the honey bee (Apis mellifera) colony collapse disorder (Evans and Schwarz, 2011), among them are the Deformed wing virus (DWV) of the Iflaviridae virus family, and Israeli acute paralysis virus (IAPV), Kashmir bee virus (KBV) and Acute bee paralysis virus (ABPV) of the Dicistroviridae family. Virulent infections by these viruses may lead to the death of entire bee colonies (de Miranda and Genersch, 2010; McMenamin and Genersch, 2015). These viruses may be found in other arthropod species (Levitt et al., 2013). The full extent of the effects of infections from these viruses are not understood in all species except bees (Apis spp.) and bumblebee (Bumbus spp.). In this dissertation, DWV was discovered to infect and actively replicate within Argentine ants in New Zealand (see Chapter 5). DWV effects on Argentine ants are not yet known. However, the virus may be one candidate to explain the decline of the ant population in New Zealand. Furthermore, these results indicate that Argentine ants are a possible additional pathway and infection reservoir for infectious bee viruses. Argentine ants may raid bee hives for their honey and brood. The contact with the bees and their environment may allow the transfer of the virus from one species to another. The virus may also be transferred from one species to another via contaminated pollen (Mazzei et al., 2014). Invasive species may therefore constitute a potential reservoir for harmful local viruses. In addition, invasive species may themselves be affected by the viruses and their population may decline.

DWV present in Argentina seemed to correspond to a different strain of the virus. From the sequencing results and analyses, Argentine ants may have been infected in New Zealand by horizontal transfer. Further sampling of Argentine ants and bees infected by DWV in Argentina, in Europe and in Australia may indicate a different DWV in these regions, and support the hypothesis of a horizontal transfer of DWV from infected local species to Argentine ants in New Zealand.

My analyses indicated that infections by the fire ant viruses SINV-1 and SINV-2, and the bee viruses IAPV, KBV and ABPV were not detected in Argentine ants from New Zealand, Argentina or Australia. Sampling in New Zealand covered most of the range of the ants in the country and support the absence of these viruses in the invasive ants. Infections by SINV-1 are unlikely as the virus appears to be specific to fire ants (Valles et al., 2007b), as potentially is SINV-2 is specific to the fire ants. Furthermore, red imported fire ants are not present in New Zealand. Infections by IAPV, KBV and ABPV may be possible in 
Argentine ants. These viruses have already been reported in arthropods including ants foraging around infected bee hives (Levitt et al., 2013). Nevertheless, additional samplings in Argentina and Australia are necessary to confirm or dismiss the absence of the viruses in Argentine ants.

A new positive strand RNA dicistrovirus, Linepithema humile virus -1 (LHUV-1), was discovered to infect and actively replicate in Argentine ants (see Chapter 5). LHUV-1 presented a wide range and was found in Argentina, Australia and New Zealand, in all sites but one in New Zealand. Phylogenetic analyses of the sequences indicated that LHUV-1 may have been co-introduced from Argentina to Australia, and then to New Zealand. Invasive species may therefore co-introduce pathogens from their home range. The results supported previous observations made in published in literature (Strauss et al., 2012; Arbetman et al., 2013). In South America, introduced bumblebees (Bombus terrestris) cointroduced the pathogenic protozoan Apicystis bombi, now affecting native bumblebee (Bombus dahlbomii) populations (Arbetman et al., 2013). Finally, as LHUV-1 is related to IAPV and KBV, two viruses involved in colony collapse disorder, LHUV-1 is an additional potential candidate to explain the Argentine ant population decline in New Zealand (Cooling et al., 2012). If LHUV-1 is indeed pathogenic, virulent and specific to the ants, the virus may be considered as a biological control for invasive Argentine ants. However, if LHUV-1 is not specific to Argentine ants, the ants would in this case be the reservoir to an additional virus that may threatened native species populations.

\subsection{Study contributions, study improvements and future directions}

\section{Study contributions}

This study represents a first step in the understanding of the bacteria and viruses in invasive population dynamics. Multiple studies documented the low genetic diversity of invasive species cause by bottlenecks during the invasion process (Dlugosh and Parker, 2008; Puillandre et al., 2008; Arundell et al., 2014). This study indicates that bacterial diversity present in invasive ant species also undergoes bottlenecks (see Chapter 2). However, invasive species seem to conserve a core of bacteria. Argentine ant conserved a few 
bacterial genera, including Lactobacillus and Gluconobacter, along their invasion pathway (see Chapter 2). Changes in bacterial diversity may affect the invasive species survival and fitness as antibiotic treatments on Argentine ants showed (see Chapters 3 and 4). Bacterial diversity, then, may be involved in invasive species population dynamics. Finally, invasive species success may leave enemies in their home range and benefits from it as stated by the enemy release hypothesis (Colautti et al., 2004; Lakeman-Fraser and Ewers, 2013). Nevertheless, invasive Argentine ants seem to have co-introduced viruses, including the new LHUV-1 (see Chapter 5). Invasive species may therefore potentially carry harmful pathogens with them and become new reservoir themselves for local viruses. Argentine ants seem to have been infected in New Zealand by DWV (see Chapter 5). The virus replicates within the ants and may potentially be transferred to other species by spillback.

\section{Study improvements and future directions}

Additional sampling of Argentine ants from the Argentine ant home range, Europe and Australia may offer a more complete understanding on bacterial diversity decline along the ant pathway from Argentina to New Zealand. Sampling during the same seasons and the use of recent collected ants may lower the implication of seasonal infection variability and genomic material degradation in the observed differences of bacteria diversity between countries.

Both DNA and RNA extracted from ant samples from different Argentina, Europe, Australia and Europe should be analysed using Illumina sequencing. Illumina sequencing would allow the detection of a large quantity of genetic material from any micro-organisms infecting Argentine ants. Other micro-organisms including viruses may have suffered from the multiple bottlenecks along the ant invasive pathway as it happened for bacteria. Other viruses may be infecting Argentine ants in its home range and invasive range. In addition, DNA and RNA sequencing may allow the comparison between bacterial diversity and viral infection loads in ants from different countries. The comparison between bacterial diversity and viral infection loads may give an insight on the role of bacterial diversity in the ant health. The additional samples from the different countries may also help supporting different infection scenarios.

Direct antibiotic treatment of the queens may highlight the possible role of bacterial diversity in Argentine ant fitness. In addition, 454 sequencing and 16S universal primers 
may be used to assess the bacterial diversity present in queens before and after the antibiotic treatments. Furthermore, the use of different antibiotics in order to specifically target Lactobacillus and Gluconobacter bacteria may offer a better understanding on the role of bacteria from these genera on Argentine ant fitness and survival. The results of these antibiotic experiments may offer a better insight on the role of bacterial diversity in Argentine ant population dynamics.

Finally, testing the virulence of DWV and LHUV-1 in Argentine ants may offer a better understanding of the role of the viruses in Argentine ant population decline in New Zealand. Colonies can be infected in the laboratory to test the modified Koch's postulates (Mokili et al., 2012; Breitschwerdt et al., 2013). Koch modified postulates are used to infer pathogenicity to an organism by isolating the organism from infected individuals, infecting healthy organisms and observing disease characteristics in these newly infected organisms. In the case of Argentine ants, healthy ants are fed with food mixed with either contaminated fluids from a bee infected by DWV or killed ants infected by LHUV-1. Laboratory infected colonies and uninfected colonies (i.e. controls) are then observed under different stress situations (i.e. different food quantity and quality), and dead ants counted daily during the experiment. Specific infection of the queens may be needed to observe the effects of the viral infections on the ant colony fitness. In addition to monitoring the colony death rate, inter-specific encounters can be realised before, during and after viral infection of the ants. The outcomes of the encounters may show a decrease in Argentine ant survival after viral infection. The results of these laboratory infections may indicate a decrease in the ant survival, fitness and encounter survival, and highlight the possible involvement of DWV and LHUV-1 in the Argentine ant population decline in New Zealand.

In addition, tests on the specificity of LHUV-1 are needed if LHUV-1 is to be considered as a potential biological control agent for Argentine ants. RNA can be extracted from arthropods found in the surrounding of Argentine ant nests. LHUV-1 presence is then screened using the primers designed in this study (Chapter 5). In parallel, infection trials are run with other arthropods that may interact with Argentine ants, such as bees. Bees are feed a food containing killed ants infected by LHUV-1. Infection and active replication of the virus may be tested at different time after the infection treatment using RT-PCR and qPCR. If no infection and replication can be find in arthropods surrounding ants and in bees infected in the laboratory, LHUV-1 could be specific to Argentine ants and considered as 
potential biological control agent. Full sequencing of LHUV-1, bee infection trials and Argentine ant colony infections are currently in progress.

Invasive species loses infections on their invasive pathway while keeping a core of bacteria. In addition, invasive species may co-introduce pathogens into their new environments and become themselves reservoir for local pathogens. This change in bacterial diversity and new infections may affect invasive species population dynamics. Furthermore, the cointroductions of pathogens may represent a threat to native species. While invasive species represent a visible threat to the biodiversity of new environments, co-introduced pathogens may be an additional invisible threat that needs to be taken into account during management programs. 


\section{References}

Abbott, K.L. 2006. Spatial dynamics of supercolonies of the invasive yellow crazy ant, Anoplolepis gracilipes, on Christmas Island, Indian Ocean. Divers. Distrib. 12: 101-110

Ahmed, M.Z., Greyvenstein, O.F.C., Erasmus, C., Welch, J.J., and Greeff, J.M. 2013. Consistently high incidence of Wolbachia in global fig wasp communities. Ecol. Entomol. 38: $147-154$

AL-Waili, N., Al Ghamdi, A., Ansari, M.J., Al-Attal, Y., Al-Mubarak, A., and Salom, K. 2013. Differences in composition of honey samples and their impact on the antimicrobial activities against drug multiresistant bacteria and pathogenic fungi. Arch. Med. Res. 44: $307-316$

Anderson, K.E., Sheehan, T.H., Eckholm, B.J., Mott, B.M., and DeGrandi-Hoffman, G. 2011. An emerging paradigm of colony health: microbial balance of the honey bee and hive (Apis mellifera). Insectes Soc. 58: 431-444

Anderson, K.E., Sheehan, T.H., Mott, B.M., Maes, P., Snyder, L., Schwan, M.R., Walton, A., Jones, B.M., and Corby-Harris, V. 2013. Microbial ecology of the hive and pollination landscape: bacterial associates from floral nectar, the alimentary tract and stored food of honey bees (Apis mellifera). PLoS One 8: e83125

Arbetman, M.P., Meeus, I., Morales, C.L., Aizen, M.A., and Smagghe, G. 2013. Alien parasite hitchhikes to Patagonia on invasive bumblebee. Biol. Invasions 15: 489-494

Arundell, K., Dunn, A., Alexander, J., Shearman, R., Archer, N., and Ironside, J.E. 2014. Enemy release and genetic founder effects in invasive killer shrimp populations of Great Britain. Biol. Invasions 1439-1451 
Ascunce, M.S., Yang, C.-C., Oakey, J., Calcaterra, L., Wu, W.-J., Shih, C.-J., Goudet, J., Ross, K.G., and Shoemaker, D. 2011. Global invasion history of the fire ant Solenopsis invicta. Science 331: 1066-1068

Asiimwe, P., Kelly, S.E., and Hunter, M.S. 2014. Symbiont infection affects whitefly dynamics in the field. Basic Appl. Ecol. 15: 507-515

Asplen, M.K., Bano, N., Brady, C.M., Desneux, N., Hopper, K.R., Malouines, C., Oliver, K.M., White, J.A., and Heimpel, G.E. 2014. Specialisation of bacterial endosymbionts that protect aphids from parasitoids. Ecol. Entomol. 39: 736-739

Auger-Rozenberg, M. a., Boivin, T., Magnoux, E., Courtin, C., Roques, A., and Kerdelhué, C. 2012. Inferences on population history of a seed chalcid wasp: invasion success despite a severe founder effect from an unexpected source population. Mol. Ecol. 21: 6086-6103

Baird, R., Woolfolk, S., and Watson, C.E. 2007. Survey of bacterial and fungal associates of black / hybrid imported fire ants from mounds in Mississippi. Southeast. Nat. 6: 615632

Barbieri, R.F., Lester, P.J., Miller, A.S., and Ryan, K.G. 2013. A neurotoxic pesticide changes the outcome of aggressive interactions between native and invasive ants. Proc. $R$. Soc. B Biol. Sci. 280: 20132157-20132157

De Barro, P.J., Hidayat, S.H., Frohlich, D., Subandiyah, S., and Ueda, S. 2008. A virus and its vector, pepper yellow leaf curl virus and Bemisia tabaci, two new invaders of Indonesia. Biol. Invasions 10: 411-433

Becerra, J.X., Venable, G.X., and Saeidi, V. 2015. Wolbachia-free heteropterans do not produce defensive chemicals or alarm pheromones. J. Chem. Ecol. 41: 593-601

Beggs, J.R., Brockerhoff, E.G., Corley, J.C., Kenis, M., Masciocchi, M., Muller, F., Rome, Q., and Villemant, C. 2011. Ecological effects and management of invasive alien Vespidae. BioControl 56: 505-526

Bertelsmeier, C., Avril, A., Blight, O., Confais, A., Diez, L., Jourdan, H., Orivel, J., Saint Germès, N., and Courchamp, F. 2015. Different behavioural strategies among seven highly invasive ant species. Biol. Invasions 17: 2491-2503

Bian, G., Xu, Y., Lu, P., Xie, Y., and Xi, Z. 2010. The endosymbiotic bacterium Wolbachia induces resistance to dengue virus in Aedes aegypti. PLoS Pathog. 6: e1000833 
Billiet, A., Meeus, I., Van Nieuwerburgh, F., Deforce, D., Wäckers, F., and Smagghe, G. 2015. Colony contact contributes to the diversity of gut bacteria in bumblebees (Bombus terrestris). Insect Sci. ) 00, 1-8, DOI 10.1111/1744-7917.12284

Blagrove, M.S.C., Arias-Goeta, C., Failloux, a.-B., and Sinkins, S.P. 2012. Wolbachia strain wMel induces cytoplasmic incompatibility and blocks dengue transmission in Aedes albopictus. Proc. Natl. Acad. Sci. 109: 255-260

Blancafort, X., and Gómez, C. 2005. Consequences of the Argentine ant, Linepithema humile (Mayr), invasion on pollination of Euphorbia characias (L.) (Euphorbiaceae). Acta Oecologica 28: 49-55

Bonning, B.C., and Miller, W.A. 2010. Dicistroviruses. Annu. Rev. Entomol. 55: 129-150

Boursaux-Eude, C., and Gross, R. 2000. New insights into symbiotic associations between ants and bacteria. Res. Microbiol. 151: 513-519

Bouwma, A.M., and Shoemaker, D. 2011. Wolbachia wSinvictaA infections in natural populations of the fire ant Solenopsis invicta: testing for phenotypic effects. J. Insect Sci. 11: 11

Brandt, M., Van Wilgenburg, E., and Tsutsui, N.D. 2009. Global-scale analyses of chemical ecology and population genetics in the invasive Argentine ant. Mol. Ecol. 18: 997-1005

Braquart-Varnier, C., Altinli, M., Pigeault, R., Chevalier, F.D., Grève, P., Bouchon, D., and Sicard, M. 2015. The mutualistic side of Wolbachia-isopod interactions: Wolbachia mediated protection against pathogenic intracellular bacteria. Front. Microbiol. 6: 1-15

Breitschwerdt, E.B., Linder, K.L., Day, M.J., Maggi, R.G., Chomel, B.B., and Kempf, V.A.J. 2013. Koch's postulates and the pathogenesis of comparative infectious disease causation associated with Bartonella species. J. Comp. Pathol. 148: 115-125

Briano, J., Calcaterra, L., and Varone, L. 2012. Fire ants (Solenopsis spp.) and their natural enemies in Southern South America. Psyche A J. Entomol. 2012: 1-19

Buckmaster, T., Dickman, C.R., and Johnston, M.J. 2014. Assessing risks to non-target species during poison baiting programs for feral cats. PLoS One 9: e107788

Bystrykh, L. V. 2012. Generalized DNA barcode design based on hamming codes. PLoS 
One 7: e36852

Caporaso, J.G., Bittinger, K., Bushman, F.D., DeSantis, T.Z., Andersen, G.L., and Knight, R. 2010a. PyNAST: a flexible tool for aligning sequences to a template alignment. Bioinformatics 26: 266-267

Caporaso, J.G., Kuczynski, J., Stombaugh, J., Bittinger, K., Bushman, F.D., Costello, E.K., Fierer, N., Peña, A.G., Goodrich, J.K., Gordon, J.I., Huttley, G.A., Kelley, S.T., Knights, D., Koenig, J.E., Ley, R.E., Lozupone, C.A., Mcdonald, D., Muegge, B.D., Pirrung, M., Reeder, J., Sevinsky, J.R., Turnbaugh, P.J., Walters, W.A., Widmann, J., Yatsunenko, T., Zaneveld, J., and Knight, R. 2010b. QIIME allows analysis of high- throughput community sequencing data. Nat. Publ. Gr. 7: 335-336

Cariveau, D.P., Elijah Powell, J., Koch, H., Winfree, R., and Moran, N.A. 2014. Variation in gut microbial communities and its association with pathogen infection in wild bumble bees (Bombus spp.). ISME J. 8: 2369-2379

Carrasco, P., Pérez-cobas, A.E., Pol, C. Van De, Baixeras, J., Moya, A., and Latorre, A. 2014. Succession of the gut microbiota in the cockroach Blattella germanica. Int. Microbiol. 17: 99-109

Cassone, B.J., Michel, A.P., Stewart, L.R., Bansal, R., Mian, M.A.R., and Redinbaugh, M.G. 2014. Reduction in fecundity and shifts in cellular processes by a native virus on an invasive insect. Genome Biol. Evol. 6: 873-885

Ceja-Navarro, J.A., Vega, F.E., Karaoz, U., Hao, Z., Jenkins, S., Lim, H.C., Kosina, P., Infante, F., Northen, T.R., and Brodie, E.L. 2015. Gut microbiota mediate caffeine detoxification in the primary insect pest of coffee. Nat. Commun. 6: 7618

Celle, O., Blanchard, P., Olivier, V., Schurr, F., Cougoule, N., Faucon, J.-P., and Ribière, M. 2008. Detection of Chronic bee paralysis virus (CBPV) genome and its replicative RNA form in various hosts and possible ways of spread. Virus Res. 133: 280-284

Chen, Y., Evans, J., and Feldlaufer, M. 2006. Horizontal and vertical transmission of viruses in the honey bee, Apis mellifera. J. Invertebr. Pathol. 92: 152-159

Chen, Y.P., Pettis, J.S., Corona, M., Chen, W.P., Li, C.J., Spivak, M., Visscher, P.K., DeGrandi-Hoffman, G., Boncristiani, H., Zhao, Y., VanEngelsdorp, D., Delaplane, K., Solter, L., Drummond, F., Kramer, M., Lipkin, W.I., Palacios, G., Hamilton, M.C., Smith, 
B., Huang, S.K., Zheng, H.Q., Li, J.L., Zhang, X., Zhou, A.F., Wu, L.Y., Zhou, J.Z., Lee, M.-L., Teixeira, E.W., Li, Z.G., and Evans, J.D. 2014. Israeli acute paralysis virus: epidemiology, pathogenesis and implications for honey bee health. PLoS Pathog. 10: e1004261

Chrostek, E., Marialva, M.S.P., Esteves, S.S., Weinert, L.A., Martinez, J., Jiggins, F.M., and Teixeira, L. 2013. Wolbachia variants induce differential protection to viruses in Drosophila melanogaster: a phenotypic and phylogenomic analysis. PLoS Genet. 9: e1003896

Clark, R.I., Salazar, A., Yamada, R., Fitz-Gibbon, S., Morselli, M., Alcaraz, J., Rana, A., Rera, M., Pellegrini, M., Ja, W.W., and Walker, D.W. 2015. Distinct shifts in microbiota composition during Drosophila aging impair intestinal function and drive mortality. Cell Rep. 12: 1656-1667

Colautti, R.I., Ricciardi, A., Grigorovich, I.A., and MacIsaac, H.J. 2004. Is invasion success explained by the enemy release hypothesis? Ecol. Lett. 7: 721-733

Colla, S.R., Otterstatter, M.C., Gegear, R.J., and Thomson, J.D. 2006. Plight of the bumble bee: pathogen spillover from commercial to wild populations. Biol. Conserv. 129: 461-467

Cook, D.C., Thomas, M.B., Cunningham, S.A., Anderson, D.L., and De Barro, P.J. 2007. Predicting the economic impact of an invasive species on an ecosystem service. Ecol. Appl. 17: $1832-1840$

Cooling, M., Hartley, S., Sim, D.A., and Lester, P.J. 2012. The widespread collapse of an invasive species: Argentine ants (Linepithema humile) in New Zealand. Biol. Lett. 8: 430433

Cooling, M., and Hoffmann, B.D. 2015. Here today, gone tomorrow: declines and local extinctions of invasive ant populations in the absence of intervention. Biol. Invasions 17: $3351-3357$

Coon, K.L., Vogel, K.J., Brown, M.R., and Strand, M.R. 2014. Mosquitoes rely on their gut microbiota for development. Mol. Ecol. 23: 2727-2739

Corby-Harris, V., Maes, P., and Anderson, K.E. 2014. The bacterial communities associated with honey bee (Apis mellifera) foragers. PLoS One 9: e95056

Corin, S.E., Abbott, K.L., Ritchie, P.A., and Lester, P.J. 2007a. Large scale unicoloniality: 
the population and colony structure of the invasive Argentine ant (Linepithema humile) in New Zealand. Insectes Soc. 54: 275-282

Corin, S.E., Lester, P.J., Abbott, K.L., and Ritchie, P.A. 2007b. Inferring historical introduction pathways with mitochondrial DNA: the case of introduced Argentine ants (Linepithema humile) into New Zealand. Divers. Distrib. 13: 510-518

Cox-Foster, D.L., Conlan, S., Holmes, E.C., Palacios, G., Evans, J.D., Moran, N.A., Quan, P.-L., Briese, T., Hornig, M., Geiser, D.M., Martinson, V., VanEngelsdorp, D., Kalkstein, A.L., Drysdale, A., Hui, J., Zhai, J., Cui, L., Hutchison, S.K., Simons, J.F., Egholm, M., Pettis, J.S., and Lipkin, W.I. 2007. A metagenomic survey of microbes in honey bee colony collapse disorder. Science 318: 283-287

Crotti, E., Rizzi, A., Chouaia, B., Ricci, I., Favia, G., Alma, A., Sacchi, L., Bourtzis, K., Mandrioli, M., Cherif, A., Bandi, C., and Daffonchio, D. 2010. Acetic acid bacteria, newly emerging symbionts of insects. Appl. Environ. Microbiol. 76: 6963-6970

Dall, D.J. 1985. Inapparent infection of honey bee pupae by Kashmir and sacbrood bee viruses in Australia. Ann. Appl. Biol. 106: 461-468

Dar, S.A., and Kotwal, S. 2014. Molecular detection and seasonal variation of Deformed wing virus affecting honey bee, Apis mellifera L. colonies. J. Entomol. Zool. Stud. 2: 295298

Degrandi-Hoffman, G., Chen, Y., Watkins Dejong, E., Chambers, M.L., and Hidalgo, G. 2015. Effects of oral exposure to fungicides on honey bee nutrition and virus levels. $J$. Econ. Entomol. 108: 2518-2528

DeSantis, T.Z., Hugenholtz, P., Larsen, N., Rojas, M., Brodie, E.L., Keller, K., Huber, T., Dalevi, D., Hu, P., and Andersen, G.L. 2006. Greengenes, a chimera-checked 16S rRNA gene database and workbench compatible with ARB. Appl. Environ. Microbiol. 72: 50695072

Dlugosh, K.M., and Parker, I.M. 2008. Founding events in species invasions: genetic variation, adaptive evolution, and the role of multiple introductions. Mol. Ecol. 17: 431449

Douglas, A.E. 1998. Nutritional interactions in insect-microbial symbioses: aphids and their symbiotic Bacteria buchnera. Annu. Rev. Entomol. 43: 17-37 
Duffy, D.C., Leader, U., Motoki, M., Lee, D.J., Vanderwoude, C., Nakamoto, S.T., and Leung, P. 2013. A bioeconomic model of little fire ant Wasmannia auropunctata in Hawaii

Dunn, A.M., and Hatcher, M.J. 2015. Parasites and biological invasions: parallels, interactions, and control. Trends Parasitol. 31: 189-199

Edelist, D., Rilov, G., Golani, D., Carlton, J.T., and Spanier, E. 2013. Restructuring the sea: profound shifts inthe world's most invaded marineecosystem. Biodivers. Distrib. 19: 6977

Edgar, R.C. 2010. Search and clustering orders of magnitude faster than BLAST. Bioinformatics 26: 2460-2461

Evans, J.D., and Schwarz, R.S. 2011. Bees brought to their knees: microbes affecting honey bee health. Trends Microbiol. 19: 614-620

Eyer, M., Chen, Y.P., Schafer, M.O., Pettis, J.S., and Neumann, P. 2009. Small hive beetle, Aethina tumida, as a potential biological vector of honeybee viruses. Apidologie 40: 419428

Fadmiro, H.Y., He, Xi., and Chen, L. 2009. Aggression in imported fire ants: an explanation for shifts in their spatial distributions in Southern United States? Ecol. Entomol. 34: 427-436

Flanagan, T.P., Pinter-Wollman, N.M., Moses, M.E., and Gordon, D.M. 2013. Fast and flexible: Argentine ants recruit from nearby trails. PLoS One 8: e70888

Flory, S.L., and Clay, K. 2013. Pathogen accumulation and long-term dynamics of plant invasions. J. Ecol. 101: 607-613

Forsgren, E., Olofsson, T.C., Vásquez, A., and Fries, I. 2009. Novel lactic acid bacteria inhibiting Paenibacillus larvae in honey bee larvae. Apidologie 41: 99-108

Francis, R., and Kryger, P. 2012. Single assay detection of Acute bee paralysis virus, Kashmir bee virus and Israeli acute paralysis virus. J. Apic. Sci. 56: 137-146

Frank, D.N. 2009. BARCRAWL and BARTAB: software tools for the design and implementation of barcoded primers for highly multiplexed DNA sequencing. BMC Bioinformatics 10: 362

Gamboa, V., Ravoet, J., Brunain, M., Smagghe, G., Meeus, I., Figueroa, J., Riaño, D., and 
de Graaf, D.C. 2015. Bee pathogens found in Bombus atratus from Colombia: a case study. J. Invertebr. Pathol. 129: 36-39

Gayatri Priya, N., Ojha, A., Kajla, M.K., Raj, A., and Rajagopal, R. 2012. Host plant induced variation in gut bacteria of Helicoverpa armigera. PLoS One 7: e30768

Genersch, E. 2010a. American Foulbrood in honeybees and its causative agent, Paenibacillus larvae. J. Invertebr. Pathol. 103 Suppl : S10-S19

Genersch, E. 2010b. Honey bee pathology: current threats to honey bees and beekeeping. Appl. Microbiol. Biotechnol. 87: 87-97

Gerth, M., Saeed, A., White, J.A., and Bleidorn, C. 2015. Extensive screen for bacterial endosymbionts reveals taxon-specific distribution patterns among bees (Hymenoptera, Anthophila). FEMS Microbiol. Ecol.

Gherman, B.I., Denner, A., Bobiş, O., Dezmirean, D.S., Mărghitaş, L.A., Schlüns, H., Moritz, R.F.A., and Erler, S. 2014. Pathogen-associated self-medication behavior in the honeybee Apis mellifera. Behav. Ecol. Sociobiol. 68: 1777-1784

Gordon, D.M., and Heller, N.E. 2014. The invasive Argentine ant Linepithema humile (Hymenoptera : Formicidae) in Northern California reserves : from foraging behavior to local spread. Myrmecological News 19: 103-110

Grosholz, E.D. 2005. Recent biological invasion may hasten invasional meltdown by accelerating historical introductions. Proc. Natl. Acad. Sci. U. S. A. 102: 1088-1091

Gruber, M.A.M., Burne, A.R., Abbott, K.L., Pierce, R.J., and Lester, P.J. 2012. Population decline but increased distribution of an invasive ant genotype on a Pacific atoll. Biol. Invasions. 15:599-612

Gunawardana, D.N., Peacock, L.R., Flynn, A.R., Ashcroft, T.T., and Green, O.R. 2013. Why is Napier sea port a hot spot for invasive ants? New Zeal. Plant Prot. 66: 10-16

Hagimori, T., Abe, Y., Date, S., and Miura, K. 2006. The first finding of a Rickettsia bacterium associated with parthenogenesis induction among insects. Curr. Microbiol. 52: $97-101$

Haines, I.H., and Haines, J.B. 1978. Pest status of the crazy ant, Anoplolepis longipes (Jerdon) (Hymenoptera: Formicidae), in the Seychelles. Bull. Entomol. Res. 68: 627 
Hamilton, P.T., and Perlman, S.J. 2013. Host defense via symbiosis in Drosophila. PLoS Pathog. 9: e1003808

Hanna, C., Foote, D., and Kremen, C. 2012. Short- and long-term control of Vespula pensylvanica in Hawaii by fipronil baiting. Pest Manag. Sci. 68: 1026-1033

Hanna, C., Foote, D., and Kremen, C. 2014. Competitive impacts of an invasive nectar thief on plant-pollinator mutualisms. Ecology 95: 1622-1632

Hanna, C., Naughton, I., Boser, C., Alarcón, R., Hung, K.-L.J., and Holway, D. 2015. Floral visitation by the Argentine ant reduces bee visitation and plant seed set. Ecology 96: 222230

Harper, G. A., and Bunbury, N. 2015. Invasive rats on tropical islands: their population biology and impacts on native species. Glob. Ecol. Conserv. 3: 607-627

Hashimoto, Y., and Valles, S.M. 2008. Infection characteristics of Solenopsis invicta virus 2 in the red imported fire ant, Solenopsis invicta. J. Invertebr. Pathol. 99: 136-140

He, H., Wei, C., and Wheeler, D.E. 2014. The gut bacterial communities associated with lab-raised and field-collected ants of Camponotus fragilis (Formicidae: Formicinae). Curr. Microbiol. 69: 292-302

Hedtke, S.M., Blitzer, E.J., Montgomery, G.A., and Danforth, B.N. 2015. Introduction of non-native pollinators can lead to trans-continental movement of bee-associated fungi. PLoS One 10: e0130560

Heller, N.E., Ingram, K.K., and Gordon, D.M. 2008. Nest connectivity and colony structure in unicolonial Argentine ants. Insectes Soc. 55: 397-403

Helms, K.R., and Vinson, S.B. 2002. Widespread association of the invasive ant Solenopsis invicta with an invasive mealybug. Ecology 83: 2425-2438

Hendry, T.A., Hunter, M.S., and Baltrus, D. a. 2014. The facultative symbiont Rickettsia protects an invasive whitefly against entomopathogenic Pseudomonas syringae strains. Appl. Environ. Microbiol. 80: 7161-7168

Hernández, N., Escudero, J.A, Millán, Á.S., González-Zorn, B., Lobo, J.M., Verdú, J.R., and Suárez, M. 2013. Culturable aerobic and facultative bacteria from the gut of the polyphagic dung beetle Thorectes lusitanicus jeckel. Insect Sci. n/a - n/a 
Highfield, A.C., El Nagar, A., Mackinder, L.C.M., Noel, L.M.-L.J., Hall, M.J., Martin, S.J., and Schroeder, D.C. 2009. Deformed wing virus implicated in overwintering honeybee colony losses. Appl. Environ. Microbiol. 75: 7212-7220

Hilgenboecker, K., Hammerstein, P., Schlattmann, P., Telschow, A., and Werren, J.H. 2008. How many species are infected with Wolbachia? A statistical analysis of current data. FEMS Microbiol. Lett. 281: 215-220

Hill, M., Holm, K., Vel, T., Shah, N.J., and Matyot, P. 2003. Impact of the invasive crazy ant Anoplolepis gracilipes on Bird Island, Seychelles. J. Biodiversity Conserv. 12: 19691984

Hoffmann, B.D. 2014. Integrating biology into invasive species management is a key principle for eradication success: the case of yellow crazy ant Anoplolepis gracilipes in northern Australia. Bull. Entomol. Res. 1-11

Hoffmann, B.D., Auina, S., and Stanley, M.C. 2014. Targeted research to improve invasive species management: yellow crazy ant Anoplolepis gracilipes in Samoa. PLoS One 9:

Holway, D.A. 1999. Competitive mechanisms underlying the displacement of native ants by the invasive Argentine ant. Ecology 80: 238-251

Hudina, S., Hock, K., and Žganec, K. 2014. The role of aggression in range expansion and biological invasions. Curr. Zool. 60: 401-409

Hulme, P.E. 2015. Invasion pathways at a crossroad: policy \& research challenges for managing alien species introductions. J. Appl. Ecol. doi: 10.1111/1365-2664.12470

Human, K.G., and Gordon, D.M. 1996. Exploitation and interference competition between the invasive Argentine ant, Linepithema humile, and native ant species. Oecologia 105: $405-412$

Huson, D.H., Auch, A.F., Qi, J., and Schuster, S.C. 2007. MEGAN analysis of metagenomic data. Genome Res. 17: 377-386

Iasur-Kruh, L., Weintraub, P.G., Mozes-Daube, N., Robinson, W.E., Perlman, S.J., and Zchori-Fein, E. 2013. Novel Rickettsiella bacterium in the leafhopper Orosius albicinctus (Hemiptera: Cicadellidae). Appl. Environ. Microbiol. 79: 4246-4252

Innerebner, G., Knief, C., and Vorholt, J. a. 2011. Protection of Arabidopsis thaliana 
against leaf-pathogenic Pseudomonas syringae by Sphingomonas strains in a controlled model system. Appl. Environ. Microbiol. 77: 3202-3210

ISAC 2006. Invasive species definition clarification and guidance white paper. Natl. Invasive Species Counc. 1-11

Ishak, H.D., Miller, J.L., Sen, R., Dowd, S.E., Meyer, E., and Mueller, U.G. 2011 a. Microbiomes of ant castes implicate new microbial roles in the fungus-growing ant Trachymyrmex septentrionalis. Sci. Rep. 1: 204

Ishak, H.D., Plowes, R., Sen, R., Kellner, K., Meyer, E., Estrada, D.A., Dowd, S.E., and Mueller, U.G. 2011b. Bacterial diversity in Solenopsis invicta and Solenopsis geminata ant colonies characterized by $16 \mathrm{~S}$ amplicon 454 pyrosequencing. Microb. Ecol. 61: 821-831

Iwase, S., Tani, S., Saeki, Y., Tuda, M., Haran, J., Skuhrovec, J., and Takagi, M. 2015. Dynamics of infection with Wolbachia in Hypera postica (Coleoptera: Curculionidae) during invasion and establishment. Biol. Invasions 17: 3639-3648

Jeschke, J.M. 2014. General hypotheses in invasion ecology. Divers. Distrib. 20: 1229 1234

Jha, C.K., and Saraf, M. 2015. Plant growth promoting Rhizobacteria (PGPR): a review. J. Agric. Res. Dev. 5: 108-119

Jiggins, F.M., and Hurst, G.D.D. 2011. Microbiology. Rapid insect evolution by symbiont transfer. Science 332: 185-186

Johansson, H., Dhaygude, K., Lindström, S., Helanterä, H., Sundström, L., and Trontti, K. 2013. A metatranscriptomic approach to the identification of microbiota associated with the ant Formica exsecta. PLoS One 8: e79777

Jolly, C.J., Shine, R., and Greenlees, M.J. 2015. The impact of invasive cane toads on native wildlife in Southern Australia. Ecol. Evol. 5: 3879-3894

Jones, S.M., van Zweden, J.S., Grüter, C., Menezes, C., Alves, D.A., Nunes-Silva, P., Czaczkes, T., Imperatriz-Fonseca, V.L., and Ratnieks, F.L.W. 2012. The role of wax and resin in the nestmate recognition system of a stingless bee, Tetragonisca angustula. Behav. Ecol. Sociobiol. 66: 1-12

Jupatanakul, N., Sim, S., and Dimopoulos, G. 2014. The insect microbiome modulates 
vector competence for arboviruses. Viruses 6: 4294-4313

Kaltenpoth, M., and Engl, T. 2014. Defensive microbial symbionts in Hymenoptera. Funct. Ecol. 28: 315-327

Kautz, S., Rubin, B.E.R., and Moreau, C.S. 2013a. Bacterial infections across the ants: frequency and prevalence of Wolbachia, Spiroplasma, and Asaia. Psyche A J. Entomol. 2013: $1-11$

Kautz, S., Rubin, B.E.R., Russell, J. a, and Moreau, C.S. 2013b. Surveying the microbiome of ants: comparing 454 pyrosequencing with traditional methods to uncover bacterial diversity. Appl. Environ. Microbiol. 79: 525-534

Kearse, M., Moir, R., Wilson, A., Stones-Havas, S., Cheung, M., Sturrock, S., Buxton, S., Cooper, A., Markowitz, S., Duran, C., Thierer, T., Ashton, B., Meintjes, P., and Drummond, A. 2012. Geneious basic: an integrated and extendable desktop software platform for the organization and analysis of sequence data. Bioinformatics 28: 1647-1649

Kellner, K., Ishak, H.D., Linksvayer, T.A., and Mueller, U.G. 2015. Bacterial community composition and diversity in an ancestral ant fungus symbiosis. FEMS Microbiol. Ecol. 126

Kelly, D.W., Paterson, R.A., Townsend, C.R., Poulin, R., and Tompkins, D.M. 2009. Parasite spillback: a neglected concept in invasion ecology? Ecology 90: 2047-2056

King, K.C., and Lively, C.M. 2012. Does genetic diversity limit disease spread in natural host populations? Heredity (Edinb). 109: 199-203

Koch, H., and Schmid-Hempel, P. 2011. Socially transmitted gut microbiota protect bumble bees against an intestinal parasite. Proc. Natl. Acad. Sci. U. S. A. 108: 19288-19292

Koehler, S., Doubský, J., and Kaltenpoth, M. 2013. Dynamics of symbiont-mediated antibiotic production reveal efficient long-term protection for beewolf offspring. Front. Zool. 10: 3

Koehler, S., and Kaltenpoth, M. 2013. Maternal and environmental effects on symbiontmediated antimicrobial defence. J. Chem. Ecol. 39: 978-988

Kojima, Y., Toki, T., Morimoto, T., Yoshiyama, M., Kimura, K., and Kadowaki, T. 2011. Infestation of Japanese native honey bees by tracheal mite and virus from non-native 
European honey bees in Japan. Microb. Ecol. 62: 895-906

Kowalski, K.P., Bacon, C., Bickford, W., Braun, H., Clay, K., Leduc-Lapierre, M., Lillard, E., McCormick, M.K., Nelson, E., Torres, M., White, J., and Wilcox, D. a. 2015. Advancing the science of microbial symbiosis to support invasive species management: a case study on Phragmites in the Great Lakes. Front. Microbiol. 6: 1-14

Lai, L.-C., Hua, K.-H., and Wu, W.-J. 2015. Intraspecific and interspecific aggressive interactions between two species of fire ants, Solenopsis geminata and S. invicta (Hymenoptera: Formicidae), in Taiwan. J. Asia. Pac. Entomol. 18: 93-98

Lakeman-Fraser, P., and Ewers, R.M. 2013. Enemy release promotes range expansion in a host plant. Oecologia 172: 1203-1212

Lee, D.J., Motoki, M., Vanderwoude, C., Nakamoto, S.T., and Leung, P. 2015. Taking the sting out of little fire ant in Hawaii. Ecol. Econ. 111: 100-110

Lee, P.O., McLellan, S.L., Graham, L.E., and Young, E.B. 2014. Invasive dreissenid mussels and benthic algae in Lake Michigan: characterizing effects on sediment bacterial communities. FEMS Microbiol. Ecol. 1-12

Lester, P., Bosch, P., Brenton-rule, E., and Corley, J. 2015a. No evidence of enemy release in pathogen and microbial communities of common wasps (Vespula vulgaris) in their native and introduced range. PLoS One. 10: e0121358. doi:10.1371/journal.pone.0121358

Lester, P.J., Baring, C.W., Longson, C.G., and Hartley, S. 2003. Argentine and other ants (Hymenoptera: Formicidae) in New Zealand horticultural ecosystems: distribution, hemipteran hosts, and review. New Zeal. Entomol. 26: 79-89

Lester, P.J., Bosch, P.J., Gruber, M. a. M., Kapp, E. a., Peng, L., Brenton-Rule, E.C., Buchanan, J., Stanislawek, W.L., Archer, M., Corley, J.C., Masciocchi, M., Van Oystaeyen, A., and Wenseleers, T. 2015b. No evidence of enemy release in pathogen and microbial communities of common wasps (Vespula vulgaris) in their native and introduced range. PLoS One 10: e0121358

Levan, K.E., Hung, K.-L.J., McCann, K.R., Ludka, J.T., and Holway, D. a 2014. Floral visitation by the Argentine ant reduces pollinator visitation and seed set in the coast barrel cactus, Ferocactus viridescens. Oecologia 174: 163-171

Levitt, A.L., Singh, R., Cox-Foster, D.L., Rajotte, E., Hoover, K., Ostiguy, N., and Holmes, 
E.C. 2013. Cross-species transmission of honey bee viruses in associated arthropods. Virus Res. 176: $232-240$

Li, H., Medina, F., Vinson, S.B., and Coates, C.J. 2005. Isolation, characterization, and molecular identification of bacteria from the red imported fire ant (Solenopsis invicta) midgut. J. Invertebr. Pathol. 89: 203-209

Liang, D., Blomquist, G.J., and Silverman, J. 2001. Hydrocarbon-released nestmate aggression in the Argentine ant, Linepithema humile, following encounters with insect prey. Comp. Biochem. Physiol. B. Biochem. Mol. Biol. 129: 871-882

Liang, D., and Silverman, J. 2000. "You are what you eat": diet modifies cuticular hydrocarbons and nestmate recognition in the Argentine ant, Linepithema humile. Naturwissenschaften 87: 412-416

Liberti, J., Sapountzis, P., Hansen, L.H., Sørensen, S.J., Adams, R.M.M., and Boomsma, J.J. 2015. Bacterial symbiont sharing in Megalomyrmex social parasites and their fungusgrowing ant hosts. Mol. Ecol. 24: 3151-3169

Lockwood, J.L., Cassey, P., and Blackburn, T. 2005. The role of propagule pressure in explaining species invasions. Trends Ecol. Evol. 20: 223-228

Lozupone, C., and Knight, R. 2005. Unifrac: a new phylogenetic method for comparing microbial communities. Appl. Environ. Microbiol. 71: 8228-8235

Lu, C., Warchol, K.M., and Callahan, R.A. 2014. Sub-lethal exposure to neonicotinoids impaired honey bees winterization before proceeding to colony collapse disorder. Bull. Insectology 67: 125-130

Lymbery, A.J., Morine, M., Kanani, H.G., Beatty, S.J., and Morgan, D.L. 2014. Coinvaders: the effects of alien parasites on native hosts. Int. J. Parasitol. Parasites Wildl. 3: $171-177$

Maistrenko, O.M., Serga, S.V, Vaiserman, A.M., and Kozeretska, I.A. 2015. Effect of Wolbachia infection on aging and longevity-associated genes in Drosophila. In: New England Journal of Medicine, pp. 83-104

Manfredini, F., Shoemaker, D., and Grozinger, C.M. 2016. Dynamic changes in host-virus interactions associated with colony founding and social environment in fire ant queens (Solenopsis invicta). Ecol. Evol. 6: 233-244 
Mansfield, J., Genin, S., Magori, S., Citovsky, V., Sriariyanum, M., and Ronald, P. 2012. Top 10 plant pathogenic bacteria in molecular plant pathology. Mol. Plant Pathol. 13: 614629

Mardis, E.R. 2008a. Next-generation DNA sequencing methods. Annu. Rev. Genomics Hum. Genet. 9: 387-402

Mardis, E.R. 2008b. The impact of next-generation sequencing technology on genetics. Trends Genet. 24: 133-141

Marsot, M., Chapuis, J.L., Gasqui, P., Dozières, A., Masséglia, S., Pisanu, B., Ferquel, E., and Vourc'h, G. 2013. Introduced Siberian chipmunks (Tamias sibiricus barberi) contribute more to Lyme borreliosis risk than native reservoir rodents. PLoS One 8: 1-8

Marsot, M., Sigaud, M., Chapuis, J.L., Ferquel, E., Cornet, M., and Vourc'h, G. 2011. Introduced Siberian chipmunks (Tamias sibiricus barberi) harbor more-diverse Borrelia burgdorferi sensu lato genospecies than native bank voles (Myodes glareolus). Appl. Environ. Microbiol. 77: 5716-5721

Martinez, J., Longdon, B., Bauer, S., Chan, Y.-S., Miller, W.J., Bourtzis, K., Teixeira, L., and Jiggins, F.M. 2014. Symbionts commonly provide broad spectrum resistance to viruses in insects: a comparative analysis of Wolbachia strains. PLoS Pathog. 10: e1004369

Martinson, V.G., Danforth, B.N., Minckley, R.L., Rueppell, O., Tingek, S., and Moran, N. a 2011. A simple and distinctive microbiota associated with honey bees and bumble bees. Mol. Ecol. 20: 619-628

Marzal, A., Ricklefs, R.E., Valkiunas, G., Albayrak, T., Arriero, E., Bonneaud, C., Czirják, G.A., Ewen, J., Hellgren, O., Hořáková, D., Iezhova, T.A., Jensen, H., Križanauskiene, A., Lima, M.R., de Lope, F., Magnussen, E., Martin, L.B., Møller, A.P., Palinauskas, V., Pap, P.L., Pérez-Tris, J., Sehgal, R.N.M., Soler, M., Szöllosi, E., Westerdahl, H., Zetindjiev, P., and Bensch, S. 2011. Diversity, loss, and gain of malaria parasites in a globally invasive bird. PLoS One 6: 1-8

Matos, R.C., and Leulier, F. 2014. Lactobacilli-host mutualism: "learning on the fly." Microb. Cell Fact. 13: S6

Matsuura, K. 2001. Nestmate recognition mediated by intestinal bacteria in a termite, Reticulitermes speratus. Oikos 92: 20-26 
Mattila, H.R., Rios, D., Walker-Sperling, V.E., Roeselers, G., and Newton, I.L.G. 2012. Characterization of the active microbiotas associated with honey bees reveals healthier and broader communities when colonies are genetically diverse. PLoS One 7: e32962

Mattoso, T.C., Moreira, D.D.O., and Samuels, R.I. 2012. Symbiotic bacteria on the cuticle of the leaf-cutting ant Acromyrmex subterraneus subterraneus protect workers from attack by entomopathogenic fungi. Biol. Lett. 8: 461-464

Mazza, G., Tricarico, E., Genovesi, P., and Gherardi, F. 2013. Biological invaders are threats to human health: an overview. Ethol. Ecol. Evol. 26: 112-129

Mazzei, M., Carrozza, M.L., Luisi, E., Forzan, M., Giusti, M., Sagona, S., Tolari, F., and Felicioli, A. 2014. Infectivity of DWV associated to flower pollen: experimental evidence of a horizontal transmission route. PLoS One 9: e113448

McDonald, D., Price, M.N., Goodrich, J., Nawrocki, E.P., DeSantis, T.Z., Probst, A., Andersen, G.L., Knight, R., and Hugenholtz, P. 2012. An improved Greengenes taxonomy with explicit ranks for ecological and evolutionary analyses of bacteria and archaea. ISME J. 6: $610-618$

McFadden, A.M.J., Tham, K.-M., Stevenson, M., Goodwin, M., Pharo, H., Taylor, B., Munro, G., Owen, K., Peacock, L., Stanslawek, W.L., and Stone, M. 2014. Israeli acute paralysis virus not detected in Apis mellifera in New Zealand in a national survey. J. Apic. Res. 53: 520-527

McFrederick, Q.S., Wcislo, W.T., Taylor, D.R., Ishak, H.D., Dowd, S.E., and Mueller, U.G. 2012. Environment or kin: whence do bees obtain acidophilic bacteria? Mol. Ecol. 21: $1754-1768$

McMenamin, A.J., and Genersch, E. 2015. Honey bee colony losses and associated viruses. Curr. Opin. Insect Sci. 8: 121-129

Medlock, J.M., Hansford, K.M., Schaffner, F., Versteirt, V., Hendrickx, G., Zeller, H., and Bortel, W. Van 2012. A review of the invasive mosquitoes in Europe: ecology, public health risks, and control options. Vector-Borne Zoonotic Dis. 12: 435-447

Meeus, I., Brown, M.J.F., De Graaf, D.C., and Smagghe, G. 2011. Effects of invasive parasites on bumble bee declines. Conserv. Biol. 25: 662-671

Meeus, I., de Miranda, J.R., de Graaf, D.C., Wäckers, F., and Smagghe, G. 2014. Effect of 
oral infection with Kashmir bee virus and Israeli acute paralysis virus on bumblebee (Bombus terrestris) reproductive success. J. Invertebr. Pathol. 121: 64-69

Mihalca, A.D. 2015. Ticks imported to Europe with exotic reptiles. Vet. Parasitol. 213: 6771

Minard, G., Tran, F.H., Van, V.T., Goubert, C., Bellet, C., Lambert, G., Kim, K.L.H., Thuy, T.H.T., Mavingui, P., and Valiente Moro, C. 2015. French invasive Asian tiger mosquito populations harbor reduced bacterial microbiota and genetic diversity compared to Vietnamese autochthonous relatives. Front. Microbiol. 6: doi: 10.3389/fmicb.2015.00970 de Miranda, J.R., Cordoni, G., and Budge, G. 2010. The Acute bee paralysis virus-Kashmir bee virus-Israeli acute paralysis virus complex. J. Invertebr. Pathol. 103 Suppl: S30-S47 de Miranda, J.R., and Genersch, E. 2010. Deformed wing virus. J. Invertebr. Pathol. 103 Suppl: S48-S61

Mokili, J.L., Rohwer, F., and Dutilh, B.E. 2012. Metagenomics and future perspectives in virus discovery. Curr. Opin. Virol. 2: 63-77

Mondet, F., de Miranda, J.R., Kretzschmar, A., Le Conte, Y., and Mercer, A.R. 2014. On the front line: quantitative virus dynamics in honeybee (Apis mellifera) colonies along a new expansion front of the parasite Varroa destructor. PLoS Pathog. 10: e1004323

Montarsi, F., Ciocchetta, S., Devine, G., Ravagnan, S., Mutinelli, F., Frangipane di Regalbono, A., Otranto, D., and Capelli, G. 2015. Development of Dirofilaria immitis within the mosquito Aedes (finlaya) koreicus, a new invasive species for Europe. Parasit. Vectors 8: 1-9

Mooney, H. a, and Cleland, E.E. 2001. The evolutionary impact of invasive species. Proc. Natl. Acad. Sci. 98: 5446-5451

Ngoagouni, C., Kamgang, B., Nakouné, E., Paupy, C., and Kazanji, M. 2015. Invasion of Aedes albopictus (Diptera: Culicidae) into central Africa: what consequences for emerging diseases? Parasit. Vectors 8: 1-7

Nguyen, D.T., Spooner-Hart, R.N., and Riegler, M. 2015. Loss of Wolbachia but not Cardinium in the invasive range of the Australian thrips species, Pezothrips kellyanus. Biol. Invasions 
O’Brien, V. a, Moore, A.T., Young, G.R., Komar, N., Reisen, W.K., and Brown, C.R. 2011. An enzootic vector-borne virus is amplified at epizootic levels by an invasive avian host. Proc. Biol. Sci. 278: 239-246

O’Dowd, D.J., Green, P.T., and Lake, P.S. 2003. Invasional "meltdown" on an oceanic island. Ecol. Lett. 6: $812-817$

Oi, D.H., and Valles, S.M. 2009. Fire ant control with entomopathogens in the USA. In: Use of Microbes for Control and Eradication of Invasive Arthropods, pp. 237-257

Palacios, G., Hui, J., Quan, P.L., Kalkstein, a, Honkavuori, K.S., Bussetti, A.V, Conlan, S., Evans, J., Chen, Y.P., vanEngelsdorp, D., Efrat, H., Pettis, J., Cox-Foster, D., Holmes, E.C., Briese, T., and Lipkin, W.I. 2008. Genetic analysis of Israel acute paralysis virus: distinct clusters are circulating in the United States. J. Virol. 82: 6209-6217

Paulson, A.R., von Aderkas, P., and Perlman, S.J. 2014. Bacterial associates of seedparasitic wasps (Hymenoptera: Megastigmus). BMC Microbiol. 14: 224

Perlman, S.J., Kelly, S.E., and Hunter, M.S. 2008. Population biology of cytoplasmic incompatibility: maintenance and spread of Cardinium symbionts in a parasitic wasp. Genetics 178: 1003-1011

Perrault, A., Bennett, M., Burgiel, S., Delach, A., and Muffett, C. 2003. Invasive species, agriculture and trade: case studies from the NAFTA context. Report: Second North American Symposium. 1-55

Pimentel, D., Zuniga, R., and Morrison, D. 2005. Update on the environmental and economic costs associated with alien-invasive species in the United States. Ecol. Econ. 52: 273-288

Porter, S.D., Valles, S.M., and Oi, D.H. 2013. Host specificity and colony impacts of the fire ant pathogen, Solenopsis invicta virus 3. J. Invertebr. Pathol. 114: 1-6

Potiwat, R., and Sitcharungsi, R. 2015. Ant allergens and hypersensitivity reactions in response to ant stings. Asian Pacific J. Allergy Immunol. 33: 267-275

Poulsen, M., Bot, a. N.M., Currie, C.R., Nielsen, M.G., and Boomsma, J.J. 2003. Withincolony transmission and the cost of a mutualistic bacterium in the leaf-cutting ant Acromyrmex octospinosus. Funct. Ecol. 17: 260-269 
Poulsen, M., and Sapountzis, P. 2012. Behind every great ant, there is a great gut. Mol. Ecol. 21: 2054-2057

Praet, J., Meeus, I., Cnockaert, M., Houf, K., Smagghe, G., and Vandamme, P. 2015. Novel lactic acid bacteria isolated from the bumble bee gut: Convivina intestini gen. nov., sp. nov., Lactobacillus bombicola sp. nov., and Weissella bombi sp. nov. Antonie Van Leeuwenhoek. 107:1337-1349

Prevéy, J.S., and Seastedt, T.R. 2015. Increased winter precipitation benefits the native plant pathogen Ustilago bullata that infects an invasive grass. Biol. Invasions 17: 30413047

Prior, K.M., Powell, T.H.Q., Joseph, A.L., and Hellmann, J.J. 2015. Insights from community ecology into the role of enemy release in causing invasion success: the importance of native enemy effects. Biol. Invasions 17: 1283-1297

Di Prisco, G., Cavaliere, V., Annoscia, D., Varricchio, P., Caprio, E., Nazzi, F., Gargiulo, G., and Pennacchio, F. 2013. Neonicotinoid clothianidin adversely affects insect immunity and promotes replication of a viral pathogen in honey bees. Proc. Natl. Acad. Sci. U. S. A. 110: $18466-18471$

Puillandre, N., Dupas, S., Dangles, O., Zeddam, J.-L., Capdevielle-Dulac, C., Barbin, K., Torres-Leguizamon, M., and Silvain, J.-F. 2008. Genetic bottleneck in invasive species: the potato tuber moth adds to the list. Biol. Invasions 10: 319-333

Rainey, S.M., Shah, P., Kohl, A., and Dietrich, I. 2014. Understanding the Wolbachiamediated inhibition of arboviruses in mosquitoes: progress and challenges. J. Gen. Virol. 95: $517-530$

Rao, R., and Weil, G.J. 2002. In vitro effects of antibiotics on Brugia malayi worm survival and reproduction. J. Parasitol. 88: 605-611

Raquin, V., Valiente Moro, C., Saucereau, Y., Tran, F.-H., Potier, P., and Mavingui, P. 2015. Native Wolbachia from Aedes albopictus blocks Chikungunya virus infection in cellulo. PLoS One 10: e0125066

Rathé, A.A., Pilkington, L.J., Spohr, L.J., Hoddle, M.S., Daugherty, M.P., and Gurr, G.M. 2015. Invasion pathway risk analysis for the glassy-winged sharpshooter (Homalodisca vitripennis): survival and reproductive success following simulated air transportation. Biol. 
Invasions 17: 2963-2973

Rathé, A.A., Pilkington, L.J., Gurr, G.M., Hoddle, M.S., Daugherty, M.P., Constable, F.E., Luck, J.E., Powell, K.S., Fletcher, M.J., and Edwards, O.R. 2012. Incursion preparedness: Anticipating the arrival of an economically important plant pathogen Xylella fastidiosa wells (Proteobacteria: Xanthomonadaceae) and the insect vector Homalodisca vitripennis (Germar) (Hemiptera: Cicadellidae) in Australia. Aust. J. Entomol. 51: 209-220

Reber, A., Castella, G., Christe, P., and Chapuisat, M. 2008. Experimentally increased group diversity improves disease resistance in an ant species. Ecol. Lett. 11: 682-689

Reuter, M., Pedersen, J.S., and Keller, L. 2005. Loss of Wolbachia infection during colonisation in the invasive Argentine ant Linepithema humile. Heredity (Edinb). 94: 364 369

Rey, O., Estoup, A., Facon, B., Loiseau, A., Aebi, A., Duron, O., Vavre, F., and Foucaud, J. 2013. Distribution of endosymbiotic reproductive manipulators reflects invasion process and not reproductive system polymorphism in the little fire ant Wasmannia auropunctata. PLoS One 8: e58467

Reynaldi, F.J., Sguazza, G.H., Albicoro, F.J., Pecoraro, M.R., and Galosi, C.M. 2013. First molecular detection of co-infection of honey bee viruses in asymptomatic Bombus atratus in South America. Braz. J. Biol. 73: 797-800

Reynaldi, F.J., Sguazza, G.H., Pecoraro, M.R., Tizzano, M.A., and Galosi, C.M. 2010. First report of viral infections that affect argentine honeybees. Environ. Microbiol. Rep. 2: 749751

Reynaldi, F.J., Sguazza, G.H., Tizzano, M. a, Fuentealba, N., Galosi, C.M., and Pecoraro, M.R. 2011. First report of Israeli acute paralysis virus in asymptomatic hives of Argentina. Rev. Argent. Microbiol. 43: 84-86

Roossinck, M.J. 2011. The good viruses: viral mutualistic symbioses. Nat. Rev. Microbiol. 9: $99-108$

Roy, H.E., Lawson Handley, L.-J., Schönrogge, K., Poland, R.L., and Purse, B. V. 2011. Can the enemy release hypothesis explain the success of invasive alien predators and parasitoids? BioControl 56: 451-468

Runckel, C., Flenniken, M.L., Engel, J.C., Ruby, J.G., Ganem, D., Andino, R., and DeRisi, 
J.L. 2011. Temporal analysis of the honey bee microbiome reveals four novel viruses and seasonal prevalence of known viruses, Nosema, and Crithidia. PLoS One 6: e20656

Ruscoe, W.A., Ramsey, D.S.L., Pech, R.P., Sweetapple, P.J., Yockney, I., Barron, M.C., Perry, M., Nugent, G., Carran, R., Warne, R., Brausch, C., and Duncan, R.P. 2011. Unexpected consequences of control: competitive vs. predator release in a four-species assemblage of invasive mammals. Ecol. Lett. 14: 1035-1042

Ruscoe, W.A., Sweetapple, P.J., Perry, M., and Duncan, R.P. 2013. Effects of spatially extensive control of invasive rats on abundance of native invertebrates in mainland New Zealand forests. Conserv. Biol. 27: 74-82

Russell, J.A, Moreau, C.S., Goldman-Huertas, B., Fujiwara, M., Lohman, D.J., and Pierce, N.E. 2009. Bacterial gut symbionts are tightly linked with the evolution of herbivory in ants. Proc. Natl. Acad. Sci. U. S. A. 106: 21236-21241

Ryabov, E. V, Wood, G.R., Fannon, J.M., Moore, J.D., Bull, J.C., Chandler, D., Mead, A., Burroughs, N., and Evans, D.J. 2014. A virulent strain of Deformed Wing Virus (DWV) of honeybees (Apis mellifera) prevails after Varroa destructor-mediated, or in vitro, transmission. PLoS Pathog. 10: e1004230

Sanders, N.J., Gotelli, N.J., Heller, N.E., and Gordon, D.M. 2003. Community disassembly by an invasive species. Proc. Natl. Acad. Sci. 100: 2474-2477

Santos, A.V., Dillon, R.J., Dillon, V.M., Reynolds, S.E., and Samuels, R.I. 2004. Ocurrence of the antibiotic producing bacterium Burkholderia sp. in colonies of the leaf-cutting ant Atta sexdens rubropilosa. FEMS Microbiol. Lett. 239: 319-323

Sassa, Y., Ono, S., Takata, M., Koyama, S., Furuya, T., Satoh, T., Ohmatsu, T., Nagai, M., Sakai, C., Mizutani, T., Hayashi, S., Moriyama, H., and Urayama, S. 2015. Identification, characterization and full-length sequence analysis of a novel dsRNA virus isolated from the arboreal ant Camponotus yamaokai. J. Gen. Virol. 96: 1930-1937

Saunders, A., and Norton, D.. 2001. Ecological restoration at mainland islands in New Zealand. Biol. Conserv. 99: 109-119

Schlüns, H., and Crozier, R.H. 2009. Molecular and chemical immune defenses in ants (Hymenoptera : Formicidae). Myrmecological News 12: 237-249

Schmid-Hempel, R., Eckhardt, M., Goulson, D., Heinzmann, D., Lange, C., Plischuk, S., 
Escudero, L.R., Salathé, R., Scriven, J.J., and Schmid-Hempel, P. 2014. The invasion of southern South America by imported bumblebees and associated parasites. J. Anim. Ecol. 83: $823-837$

Scully, E.D., Geib, S.M., Hoover, K., Tien, M., Tringe, S.G., Barry, K.W., Glavina del Rio, T., Chovatia, M., Herr, J.R., and Carlson, J.E. 2013. Metagenomic profiling reveals lignocellulose degrading system in a microbial community associated with a wood-feeding beetle. PLoS One 8: e73827

Sébastien, A., Gruber, M.A. M., and Lester, P.J. 2011. Prevalence and genetic diversity of three bacterial endosymbionts (Wolbachia, Arsenophonus, and Rhizobiales) associated with the invasive yellow crazy ant (Anoplolepis gracilipes). Insectes Soc. 59: 33-40

Sébastien, A., Lester, P.J., Hall, R.J., Wang, J., Moore, N.E., and Gruber, M.A.M. 2015. Invasive ants carry novel viruses in their new range and form reservoirs for a honeybee pathogen. Biol. Lett. 11: 20150610

Seipel, T., Kueffer, C., Rew, L.J., Daehler, C.C., Pauchard, A., Naylor, B.J., Alexander, J.M., Edwards, P.J., Parks, C.G., Arevalo, J.R., Cavieres, L. a., Dietz, H., Jakobs, G., Mcdougall, K., Otto, R., and Walsh, N. 2012. Processes at multiple scales affect richness and similarity of non-native plant species in mountains around the world. Glob. Ecol. Biogeogr. 21: 236-246

Shackleton, R.T., Le Maitre, D.C., Pasiecznik, N.M., and Richardson, D.M. 2014. Prosopis: a global assessment of the biogeography, benefits, impacts and management of one of the world's worst woody invasive plant taxa. AoB Plants 6: plu027-plu027

Sheath, D.J., Williams, C.F., Reading, A.J., and Britton, J.R. 2015. Parasites of non-native freshwater fishes introduced into England and Wales suggest enemy release and parasite acquisition. Biol. Invasions 17: 2235-2246

Shen, M. 2005. Intricate transmission routes and interactions between picorna-like viruses (Kashmir bee virus and Sacbrood virus) with the honeybee host and the parasitic Varroa mite. J. Gen. Virol. 86: 2281-2289

Shi, X., Pan, H., Xie, W., Jiao, X., Fang, Y., Chen, G., Yang, X., Wu, Q., Wang, S., and Zhang, Y. 2014. Three-way interactions between the tomato plant, tomato yellow leaf curl virus, and Bemisia tabaci (Hemiptera: Aleyrodidae) facilitate virus spread. J. Econ. 
Entomol. 107: 920-926

Simberloff, D., and Gibbons, L. 2004. Now you see them, now you don't! Population crashes of established introduced species. Biol. Invasions 6: 161-172

Simberloff, D., Martin, J.-L., Genovesi, P., Maris, V., Wardle, D.A., Aronson, J., Courchamp, F., Galil, B., García-Berthou, E., Pascal, M., Pyšek, P., Sousa, R., Tabacchi, E., and Vilà, M. 2013. Impacts of biological invasions: what's what and the way forward. Trends Ecol. Evol. 28: 58-66

Singh, R., Levitt, A.L., Rajotte, E.G., Holmes, E.C., Ostiguy, N., VanEngelsdorp, D., Lipkin, W.I., DePamphilis, C.W., Toth, A.L., and Cox-Foster, D.L. 2010. RNA viruses in hymenopteran pollinators: evidence of inter-Taxa virus transmission via pollen and potential impact on non-Apis hymenopteran species. PLoS One 5: e14357

Sirviö, A., and Pamilo, P. 2010. Multiple endosymbionts in populations of the ant Formica cinerea. BMC Evol. Biol. 10: 335

Sloan, D.B., and Moran, N.A, $\backslash 2012$. Endosymbiotic bacteria as a source of carotenoids in whiteflies. Biol. Lett. 8: 986-989

Smith, C.C., and Mueller, U.G. 2015. Sexual transmission of beneficial microbes. Trends Ecol. Evol. 30: 438-440

de Souza, D.J., Bézier, A., Depoix, D., Drezen, J.-M., and Lenoir, A. 2009. Blochmannia endosymbionts improve colony growth and immune defence in the ant Camponotus fellah. BMC Microbiol. 9: 29

Stankus, T. 2008. A review and bibliography of the literature of honey bee colony collapse disorder: a poorly understood epidemic that clearly threatens the successful pollination of billions of dollars of crops in America. J. Agric. Food Inf. 9: 115-143

Stoll, S., Gadau, J., Gross, R., and Feldhaar, H. 2007. Bacterial microbiota associated with ants of the genus Tetraponera. Biol. J. Linn. Soc. 90: 399-412

Stoops, J., Crauwels, S., Waud, M., Claes, J., Lievens, B., and Van Campenhout, L. 2016. Microbial community assessment of mealworm larvae (Tenebrio molitor) and grasshoppers (Locusta migratoria migratorioides) sold for human consumption. Food Microbiol. 53: $122-127$ 
Storelli, G., Defaye, A., Erkosar, B., Hols, P., Royet, J., and Leulier, F. 2011. Lactobacillus plantarum promotes drosophila systemic growth by modulating hormonal signals through TOR-dependent nutrient sensing. Cell Metab. 14: 403-414

Strand, M.R., and Burke, G.R. 2013. Polydnavirus-wasp associations: evolution, genome organization, and function. Curr. Opin. Virol. 3: 587-594

Strauss, A., White, A., and Boots, M. 2012. Invading with biological weapons: the importance of disease-mediated invasions. Funct. Ecol. 26: 1249-1261

Strunov, A., and Kiseleva, E. 2015. Drosophila melanogaster brain invasion: pathogenic Wolbachia in central nervous system of the fly. Insect Sci. 7: doi: 10.1111/17447917.12187

Su, Q., Oliver, K.M., Pan, H., Jiao, X., Liu, B., Xie, W., Wang, S., Wu, Q., Xu, B., White, J. a, Zhou, X., and Zhang, Y. 2013. Facultative symbiont Hamiltonella confers benefits to Bemisia tabaci (Hemiptera: Aleyrodidae), an invasive agricultural pest worldwide. Environ. Entomol. 42: 1265-1271

Suarez, A.V, Holway, D.A., and Case, T.J. 2001. Patterns of spread in biological invasions dominated by long-distance jump dispersal: insights from Argentine ants. Proc. Natl. Acad. Sci. 98: $1095-1100$

Suarez, A.V, Tsutsui, N.D., Holway, D.A., and Case, T.J. 1999. Behavioral and genetic differentiation between native and introduced populations of the Argentine ant. Biol. Invasions 1: 43-53

Suen, G., Scott, J.J., Aylward, F.O., Adams, S.M., Tringe, S.G., Pinto-Tomás, A.A., Foster, C.E., Pauly, M., Weimer, P.J., Barry, K.W., Goodwin, L.A., Bouffard, P., Li, L., Osterberger, J., Harkins, T.T., Slater, S.C., Donohue, T.J., and Currie, C.R. 2010. An insect herbivore microbiome with high plant biomass-degrading capacity. PLoS Genet. 6: e1001129

Suhr, E.L., O’Dowd, D.J., McKechnie, S.W., and Mackay, D.A. 2011. Genetic structure, behaviour and invasion history of the Argentine ant supercolony in Australia. Evol. Appl. 4: $471-484$

Tada, A., Kikuchi, Y., Hosokawa, T., Musolin, D.L., Fujisaki, K., and Fukatsu, T. 2011. Obligate association with gut bacterial symbiont in Japanese populations of the Southern 
green stinkbug Nezara viridula (Heteroptera: Pentatomidae). Appl. Entomol. Zool. 46: 483488

Tajabadi, N., Mardan, M., Mustafa, S., Feizabadi, F., Nateghi, L., Rasti, B., Yazid, M., and Manap, A. 2012. Weissella sp . Taj-Apis, a novel lactic acid bacterium isolated from honey. J. Food, Agric. Environ. 10: 263-267

Tang, X., Adler, P.H., Vogel, H., and Ping, L. 2012. Gender-specific bacterial composition of black flies (Diptera: Simuliidae). FEMS Microbiol. Ecol. 80: 659-670

Teixeira, L., Ferreira, Á., and Ashburner, M. 2008. The bacterial symbiont Wolbachia induces resistance to RNA viral infections in Drosophila melanogaster. PLoS Biol. 6: $\mathrm{e} 1000002$

Tentcheva, D., Gauthier, L., Zappulla, N., Dainat, B., Cousserans, F., Edouard, M., Bergoin, M., and Colin, M.E. 2004. Prevalence and seasonal variations of six bee viruses in Apis mellifera L. and Varroa destructor mite populations in France. Appl. Environ. Microbiol. 70: 7185-7191

Tinsley, R.C., Stott, L.C., Viney, M.E., Mable, B.K., and Tinsley, M.C. 2015. Extinction of an introduced warm-climate alien species, Xenopus laevis, by extreme weather events. Biol. Invasions 17: 3183-3195

Todd, J.H., De Miranda, J.R., and Ball, B. V. 2007. Incidence and molecular characterization of viruses found in dying New Zealand honey bee (Apis mellifera) colonies infested with Varroa destructor. Apidologie 38: 354-367

Touret, F., Guiguen, F., and Terzian, C. 2014. Wolbachia influences the maternal transmission of the gypsy endogenous retrovirus in Drosophila melanogaster. MBio 5: e01529-14-e01529-14

Tragust, S., Feldhaar, H., Espadaler, X., and Pedersen, J.S. 2015. Rapid increase of the parasitic fungus Laboulbenia formicarum in supercolonies of the invasive garden ant Lasius neglectus. Biol. Invasions 17: 2795-2801

Tsuchida, K., Kudô, K., and Ishiguro, N. 2014. Genetic structure of an introduced paper wasp, Polistes chinensis antennalis (Hymenoptera, Vespidae) in New Zealand. Mol. Ecol. 23: $4018-4034$

Tsutsui, N.D., Kauppinen, S.N., Oyafuso, A.F., and Grosberg, R.K. 2003. The distribution 
and evolutionary history of Wolbachia infection in native and introduced populations of the invasive Argentine ant (Linepithema humile). Mol. Ecol. 12: 3057-3068

Tsutsui, N.D., Suarez, A.V, Holway, D.A., and Case, T.J. 2000. Reduced genetic variation and the success of an invasive species. Proc. Natl. Acad. Sci. 97: 5948-5953

Tufts, D.M., Hunter, W.B., and Bextine, B. 2010. Discovery and effects of Texas Solenopsis invicta virus [SINV-1 (TX5)] on red imported fire ant populations. J. Invertebr. Pathol. 104: 180-185

Turgis, S.J.S., and Ordon, D.M.G. 2012. Nestmate recognition in ants (Hymenoptera: Formicidae): a review. Myrmecological News 16: 101-110

Untergasser, A., Cutcutache, I., Koressaar, T., Ye, J., Faircloth, B.C., Remm, M., and Rozen, S.G. 2012. Primer3-new capabilities and interfaces. Nucleic Acids Res. 40: 1-12

Untergasser, A., Nijveen, H., Rao, X., Bisseling, T., Geurts, R., and Leunissen, J.A.M. 2007. Primer3Plus, an enhanced web interface to Primer3. Nucleic Acids Res. 35: W71W74

Vale, P.F., and Jardine, M.D. 2015. Sex-specific behavioural symptoms of viral gut infection and Wolbachia in Drosophila melanogaster. J. Insect Physiol. 82: 28-32

Valles, S.M., and Hashimoto, Y. 2009. Isolation and characterization of Solenopsis invicta virus 3, a new positive-strand RNA virus infecting the red imported fire ant, Solenopsis invicta. Virology 388: 354-361

Valles, S.M., and Oi, D.H. 2014. 2016. Novel Nylanderia fulva virus. US Patent 20160113287

Valles, S.M., Oi, D.H., and Porter, S.D. 2010. Seasonal variation and the co-occurrence of four pathogens and a group of parasites among monogyne and polygyne fire ant colonies. Biol. Control 54: 342-348

Valles, S.M., Oi, D.H., Yu, F., Tan, X.-X., and Buss, E. a 2012. Metatranscriptomics and pyrosequencing facilitate discovery of potential viral natural enemies of the invasive Caribbean crazy ant, Nylanderia pubens. PLoS One 7: e31828

Valles, S.M., and Porter, S.D. 2015. Dose response of red imported fire ant colonies to Solenopsis invicta virus 3. Arch. Virol. 160: 2407-2413 
Valles, S.M., Porter, S.D., Choi, M.-Y., and Oi, D.H. 2013a. Successful transmission of Solenopsis invicta virus 3 to Solenopsis invicta fire ant colonies in oil, sugar, and cricket bait formulations. J. Invertebr. Pathol. 113: 198-204

Valles, S.M., Porter, S.D., and Firth, A.E. 2014. Solenopsis invicta virus 3: Pathogenesis and stage specificity in red imported fire ants. Virology 460-461: 66-71

Valles, S.M., Shoemaker, D., Wurm, Y., Strong, C.A., Varone, L., Becnel, J.J., and Shirk, P.D. 2013b. Discovery and molecular characterization of an ambisense densovirus from South American populations of Solenopsis invicta. Biol. Control 67: 431-439

Valles, S.M., and Strong, C. a 2005. Solenopsis invicta virus-1A (SINV-1A): distinct species or genotype of SINV-1? J. Invertebr. Pathol. 88: 232-237

Valles, S.M., Strong, C.A., Dang, P.M., Hunter, W.B., Pereira, R.M., Oi, D.H., Shapiro, A.M., and Williams, D.F. 2004. A picorna-like virus from the red imported fire ant, Solenopsis invicta: initial discovery, genome sequence, and characterization. Virology 328 : $151-157$

Valles, S.M., Strong, C.A., and Hashimoto, Y. 2007a. A new positive-strand RNA virus with unique genome characteristics from the red imported fire ant, Solenopsis invicta. Virology 365: 457-463

Valles, S.M., Strong, C.A., Oi, D.H., Porter, S.D., Pereira, R.M., Vander Meer, R.K., Hashimoto, Y., Hooper-Bùi, L.M., Sánchez-Arroyo, H., Davis, T., Karpakakunjaram, V., Vail, K.M., "Fudd" Graham, L.C., Briano, J.A., Calcaterra, L.A., Gilbert, L.E., Ward, R., Ward, K., Oliver, J.B., Taniguchi, G., and Thompson, D.C. 2007b. Phenology, distribution, and host specificity of Solenopsis invicta virus-1. J. Invertebr. Pathol. 96: 18-27

Valles, S.M., Varone, L., Ramírez, L., and Briano, J. 2009. Multiplex detection of Solenopsis invicta viruses -1, -2, and -3. J. Virol. Methods 162: 276-279

vanEngelsdorp, D., and Meixner, M.D. 2010. A historical review of managed honey bee populations in Europe and the United States and the factors that may affect them. $J$. Invertebr. Pathol. 103: S80-S95

Vásquez, A., Forsgren, E., Fries, I., Paxton, R.J., Flaberg, E., Szekely, L., and Olofsson, T.C. 2012. Symbionts as major modulators of insect health: lactic acid bacteria and honeybees. PLoS One 7: 
Vilcinskas, A. 2015. Pathogens as biological weapons of invasive species. PLOS Pathog. 11: e1004714

Vilcinskas, A., Stoecker, K., Schmidtberg, H., Rohrich, C.R., and Vogel, H. 2013. Invasive harlequin ladybird carries biological weapons against native competitors. Science. 340: $862-863$

Viljakainen, L., Reuter, M., and Pamilo, P. 2008. Wolbachia transmission dynamics in Formica wood ants. BMC Evol. Biol. 8: 55

Vogel, V., Pedersen, J.S., Giraud, T., Krieger, M.J.B., and Keller, L. 2010. The worldwide expansion of the Argentine ant. Divers. Distrib. 16: 170-186

Vonshak, M., Dayan, T., and Hefetz, A. 2011. Interspecific displacement mechanisms by the invasive little fire ant Wasmannia auropunctata. Biol. Invasions 14: 851-861

Wagner, V., and Nelson, C.R. 2014. Herbicides can negatively affect seed performance in native plants. Restor. Ecol. 22: 288-291

Walsh, D.B., Bolda, M.P., Goodhue, R.E., Dreves, A.J., Lee, J., Bruck, D.J., Walton, V.M., O’Neal, S.D., and Zalom, F.G. 2011. Drosophila suzukii (Diptera: Drosophilidae): invasive pest of ripening soft fruit expanding its geographic range and damage potential. J. Integr. Pest Manag. 2: 1-7

Wang, Q., Garrity, G.M., Tiedje, J.M., and Cole, J.R. 2007. Naive Bayesian classifier for rapid assignment of rRNA sequences into the new bacterial taxonomy. Appl. Environ. Microbiol. 73: 5261-5267

Wang, Y., and Zhang, Y. 2015. Investigation of gut-associated bacteria in Tenebrio molitor (Coleoptera: Tenebrionidae) larvae using culture-dependent and DGGE methods. Ann. Entomol. Soc. Am. 108: 941-949

Ward, D.F., Green, C., Harris, R.J., Hartley, S., Lester, P.J., Stanley, M.C., Suckling, D.M., and Toft, R.J. 2010. Twenty years of Argentine ants in New Zealand: past research and future priorities for applied management. New Zeal. Entomol. 33: 68-78

Weinert, L. a, Werren, J.H., Aebi, A., Stone, G.N., and Jiggins, F.M. 2009. Evolution and diversity of Rickettsia bacteria. BMC Biol. 7: 6

Wenseleers, T., Sundström, L., and Billen, J. 2002. Deleterious Wolbachia in the ant 
Formica truncorum. Proc. Biol. Sci. 269: 623-629

Westermann, F.L., Bell, V. a, Suckling, D.M., and Lester, P.J. 2015. Synthetic pheromones as a management technique - dispensers reduce Linepithema humile activity in a commercial vineyard. Pest Manag. Sci. 72: 719-724, doi: 10.1002/ps.4043

Wetterer, J.K., Wild, A.L., Suarez, A. V, Roura- Pascual, N., and Espadaler, X. 2009. Worldwide spread of the Argentine ant, Linepithema humile (Hymenoptera: Formicidae). Myrmecological News 12: 187-194

Wood, J.R., Dickie, I. a., Moeller, H. V., Peltzer, D. a., Bonner, K.I., Rattray, G., and Wilmshurst, J.M. 2015. Novel interactions between non-native mammals and fungi facilitate establishment of invasive pines. J. Ecol. 103: 121-129

Xu, P., Liu, Y., Graham, R.I., Wilson, K., and Wu, K. 2014. Densovirus is a mutualistic symbiont of a global crop pest (Helicoverpa armigera) and protects against a baculovirus and Bt biopesticide. PLoS Pathog. 10: e1004490

Yamanaka, T., Morimoto, N., Nishida, G.M., Kiritani, K., Moriya, S., and Liebhold, A.M. 2015. Comparison of insect invasions in North America, Japan and their islands. Biol. Invasions 17: 3049-3061

Yang, B., Peng, G., Li, T., and Kadowaki, T. 2013. Molecular and phylogenetic characterization of honey bee viruses, Nosema microsporidia, protozoan parasites, and parasitic mites in China. Ecol. Evol. 3: 298-311

Yang, C.-C., Yu, Y.-C., Valles, S.M., Oi, D.H., Chen, Y.-C., Shoemaker, D., Wu, W.-J., and Shih, C.-J. 2010. Loss of microbial (pathogen) infections associated with recent invasions of the red imported fire ant Solenopsis invicta. Biol. Invasions 12: 3307-3318

Yang, C.-Y., Xiao, J.-H., Niu, L.-M., Ma, G.-C., Cook, J.M., Bian, S.-N., Fu, Y.-G., and Huang, D.-W. 2012. Chaos of Wolbachia Sequences inside the compact fig syconia of Ficus benjamina (Ficus: Moraceae). PLoS One 7: e48882

Yoshiyama, M., and Kimura, K. 2009. Bacteria in the gut of Japanese honeybee, Apis cerana japonica, and their antagonistic effect against Paenibacillus larvae, the causal agent of American foulbrood. J. Invertebr. Pathol. 102: 91-96

Yoshiyama, M., Wu, M., Sugimura, Y., Takaya, N., Kimoto-Nira, H., and Suzuki, C. 2013. Inhibition of Paenibacillus larvae by lactic acid bacteria isolated from fermented materials. 
J. Invertebr. Pathol. 112: 62-67

Yue, C., and Genersch, E. 2005. RT-PCR analysis of Deformed wing virus in honeybees (Apis mellifera) and mites (Varroa destructor). J. Gen. Virol. 86: 3419-3424

Zerbino, D.R., and Birney, E. 2008. Velvet: Algorithms for de novo short read assembly using de Bruijn graphs. Genome Res. 18: 821-829

Zientz, E., Feldhaar, H., Stoll, S., and Gross, R. 2005. Insights into the microbial world associated with ants. Arch. Microbiol. 184: 199-206

Zug, R., and Hammerstein, P. 2015. Bad guys turned nice? A critical assessment of Wolbachia mutualisms in arthropod hosts. Biol. Rev. 90: 89-111 
Appendix 


\section{Chapter 2: additional materials and methods - sample sites}

Table I.1: Argentine ant sample collections and ant pool composition for $16 \mathrm{~S}$ analysis. Samples from Argentina were part of an existing collection conserved in $80-100 \%$ ethanol and stored at $20^{\circ} \mathrm{C}$. The other samples were collected for this study and conserved in $100 \%$ ethanol and stored at $-20^{\circ} \mathrm{C}$. Each analysed sample composed a pool of 30 ants from the same site. The specific number of ants taken from each collection tubes to realise the pool of 30 ants is indicated in Table 2.1.

\begin{tabular}{|c|c|c|c|c|c|c|c|}
\hline Sample ID & $\begin{array}{c}\text { Number } \\
\text { of ants }\end{array}$ & $\begin{array}{c}\text { Collection } \\
\text { date }\end{array}$ & Country & State/Province & Location & Latitude & Longitude \\
\hline \multirow{3}{*}{ Arg.BA.2002 } & 5 & 2002 & Argentina & Buenos Aires & Buenos Aires & -34.6089 & -58.3574 \\
\hline & 12 & 2002 & Argentina & Buenos Aires & Buenos Aires & -34.6089 & -58.3574 \\
\hline & 13 & 2002 & Argentina & Buenos Aires & Buenos Aires & -34.6089 & -58.3574 \\
\hline \multirow{7}{*}{ Arg.ER.1999 } & 4 & 1999 & Argentina & Entre Rios & Pre Delta National Park & -32.1167 & -60.6333 \\
\hline & 4 & 1999 & Argentina & Entre Rios & Pre Delta National Park & -32.1167 & -60.6333 \\
\hline & 4 & 1999 & Argentina & Entre Rios & Pre Delta National Park & -32.1167 & -60.6333 \\
\hline & 4 & 1999 & Argentina & Entre Rios & Pre Delta National Park & -32.1167 & -60.6333 \\
\hline & 4 & 1999 & Argentina & Entre Rios & Pre Delta National Park & -32.1167 & -60.6333 \\
\hline & 5 & 1999 & Argentina & Entre Rios & Pre Delta National Park & -32.1167 & -60.6333 \\
\hline & 5 & 1999 & Argentina & Entre Rios & Pre Delta National Park & -32.1167 & -60.6333 \\
\hline \multirow{2}{*}{ Arg.Mis.1999 } & 15 & 1999 & Argentina & Missiones & Ibate & -27.4167 & -57.166667 \\
\hline & 15 & 1999 & Argentina & Missiones & Ibate & -27.4167 & -57.166667 \\
\hline \multirow{2}{*}{ Arg.Cor.1999 } & 12 & 1999 & Argentina & Corrientes & Port Alvear & -29.1 & -56.55 \\
\hline & 18 & 1999 & Argentina & Corrientes & Port Alvear & -29.1 & -56.55 \\
\hline \multirow{5}{*}{ USA.На.2002 } & 6 & 2002 & USA & Hawaii & Hawaii & 19.6869 & -155.464 \\
\hline & 6 & 2002 & USA & Hawaii & Hawaii & 19.4287 & -155.282 \\
\hline & 6 & 2002 & USA & Hawaii & Hawaii & 19.4303 & -155.258 \\
\hline & 6 & 2002 & USA & Hawaii & Hawaii & 19.4218 & -155.29 \\
\hline & 6 & 2002 & USA & Hawaii & Maui & 20.7601 & -156.246 \\
\hline \multirow{3}{*}{ USA.Sc.1999 } & 10 & 1999 & USA & California & Santa Clara County & 37.4301 & -122.169 \\
\hline & 10 & 1999 & USA & California & Santa Clara County & 37.4301 & -122.169 \\
\hline & 10 & 1999 & USA & California & Santa Clara County & 37.4433 & -122.166 \\
\hline \multirow{3}{*}{ USA.No.1999 } & 10 & 1999 & USA & Louisiana & New Orleans & 30.0389 & -90.2593 \\
\hline & 10 & 1999 & USA & Louisiana & New Orleans & 30.0389 & -90.2593 \\
\hline & 10 & 1999 & USA & California & Santa Clara County & 37.4301 & -122.169 \\
\hline USA.Cal.2011 & 30 & 2011 & USA & California & Davis & 38.5366 & -121.757 \\
\hline Oz.Mel.2012 & 30 & 2012 & Australia & (NA) & Melbourne & -37.7908 & 144.9568 \\
\hline \multirow{3}{*}{ AK.2011 } & 10 & 2011 & New Zealand & North Island & Auckland & -36.9376 & 174.4617 \\
\hline & 10 & 2011 & New Zealand & North Island & Auckland & -36.9404 & 174.4637 \\
\hline & 10 & 2011 & New Zealand & North Island & Auckland & -36.9478 & 174.4675 \\
\hline \multirow{3}{*}{ AK.2012 } & 10 & 2012 & New Zealand & North Island & Auckland & -36.9376 & 174.4617 \\
\hline & 10 & 2012 & New Zealand & North Island & Auckland & -36.9404 & 174.4637 \\
\hline & 10 & 2012 & New Zealand & North Island & Auckland & -36.9478 & 174.4675 \\
\hline \multirow{2}{*}{ WN.2011 } & 15 & 2011 & New Zealand & North Island & Wellington & -41.2231 & 174.871 \\
\hline & 15 & 2011 & New Zealand & North Island & Wellington & -41.2167 & 174.8943 \\
\hline \multirow{2}{*}{ WN.2012 } & 15 & 2012 & New Zealand & North Island & Wellington & -41.2231 & 174.871 \\
\hline & 15 & 2012 & New Zealand & North Island & Wellington & -41.2167 & 174.8943 \\
\hline HA. 2011 & 15 & 2011 & New Zealand & North Island & Hastings & -39.6269 & 176.864 \\
\hline
\end{tabular}




\begin{tabular}{c|c|c|c|c|c|c|c} 
& 15 & 2011 & New Zealand & North Island & Hastings & -39.6257 & 176.8594 \\
\hline \multirow{4}{*}{ CH.2011 } & 2 & 2011 & New Zealand & South Island & Christchurch & -43.5387 & 172.7009 \\
\cline { 2 - 7 } & 19 & 2011 & New Zealand & South Island & Christchurch & -43.5367 & 172.6353 \\
\cline { 2 - 8 } & 19 & 2011 & New Zealand & South Island & Christchurch & -43.5353 & 172.6337 \\
\hline \multirow{4}{*}{ CH.2012 } & 10 & 2012 & New Zealand & South Island & Christchurch & -43.5387 & 172.7009 \\
\cline { 2 - 8 } & 10 & 2012 & New Zealand & South Island & Christchurch & -43.5367 & 172.6353 \\
\cline { 2 - 8 } & 10 & 2012 & New Zealand & South Island & Christchurch & -43.5353 & 172.6337 \\
\hline \multirow{4}{*}{ NN.2011 } & 10 & 2011 & New Zealand & South Island & Nelson & -41.2967 & 173.2288 \\
\cline { 2 - 8 } & 10 & 2011 & New Zealand & South Island & Nelson & -41.3107 & 173.2486 \\
\hline & 10 & 2011 & New Zealand & South Island & Nelson & -41.3055 & 173.2505 \\
\hline \multirow{3}{*}{ NN.2012 } & 10 & 2012 & New Zealand & South Island & Nelson & -41.2967 & 173.2288 \\
\cline { 2 - 8 } & 10 & 2012 & New Zealand & South Island & Nelson & -41.3107 & 173.2486 \\
\cline { 2 - 8 } & 10 & 2012 & New Zealand & South Island & Nelson & -41.3055 & 173.2505
\end{tabular}




\section{Chapter 2: additional materials and methods - density measurements}

In order to separate samples in New Zealand between high and low density sites, Argentine ant densities were measured using honey baits to compare the results with a previous density assessment of Argentine ant density in New Zealand (Cooling et al., 2012). Each site consisted of three to four bait plots depending on space limits. Plots were separated by at least $10 \mathrm{~m}$. A plot consisted of three bait cards $(5.5 \times 9.0 \mathrm{~cm}$ laminated paper plate cut card with a $5.5 \times 4.5 \mathrm{~cm}$ drawn delimitation) placed on a $3 \mathrm{~m}$ long line. Card were dispersed $1 \mathrm{~m}$ apart. On the centre of each card, approximatively $5 \mathrm{~mL}$ of honey (Arataki Liquid Honey Squeeze Me) was placed. Pictures were taken every 15 min during one hour and photographed ants were counted to measure worker recruitment. Ants inside the delimitations were counted for in every picture. My observations at the sites suggested that the measurements did not appear to be reliable. In one site from Auckland (New Zealand), thousands of ants could be seen foraging but none came investigating the baits. In contrary, sites in Christchurch (New Zealand) for examples with no apparent foraging trails prior to the plotting of baits were visited by a few hundred ants. As these baiting results may reflect more the variable food preferences of the ants than the ant density, density measures were not further considered. 


\section{Chapter 4: additional materials and methods - additional antibiotic}

Rifampicin was considered as an additional antibiotic treatment because of its actions in the bacteria that differed from the three other selected antibiotics (ampicillin, gentamicin and kanamycin). Rifampicin is a bactericidal antibiotic that inhibits the synthesis of RNA in bacteria. Colonies $(\mathrm{n}=10)$ were offered $200 \mu \mathrm{L}$ of a $20 \%(\mathrm{w} / \mathrm{v})$ sucrose $-5.0 \mathrm{mg} / \mathrm{mL}$ rifampicin mix (final concentrations), on a $2 \mathrm{~cm}^{2}$ tin foil cut and observed as described in Chapter 4 (see section 4.3.2). The few ants observed within the vicinity of the mix would actively avoid the mixture after antenna or labial contact. The antibiotic mix was removed and the colonies were presented with $200 \mu \mathrm{L}$ of a $20 \%(\mathrm{w} / \mathrm{v})$ sucrose mixed with $2.5 \mathrm{mg} / \mathrm{mL}$ rifampicin (final concentrations). The same observations were made, the ants avoided the mix. The antibiotic mix was removed, and the ants were presented $200 \mu \mathrm{L}$ of a $20 \%(\mathrm{w} / \mathrm{v})$ sucrose solution. The ants came and recruited to the feeding station. This result indicated that even if hungry, the ants would not feed on a mix containing rifampicin. Rifampicin was therefore discarded as an antibiotic treatment. 


\section{Chapter 5: additional materials and methods - collection sites}

Table IV.1: Collection sites to map the range of viruses of interest in Argentine ants. Samples were collected with aspirators between 2012 and 2015. Samples were conserved in 100\% ethanol and in Ambion RNAlater (Life Technologies) at $-20^{\circ} \mathrm{C}$ for the samples from New Zealand and Argentine or at $4^{\circ} \mathrm{C}$ for the samples from Australia. One sample (Gisborne, New Zealand) was composed of ants frozen alive and conserved at $-80^{\circ} \mathrm{C}$.

\begin{tabular}{|c|c|c|c|}
\hline Country & Area / City & latitude & longitude \\
\hline \multirow{27}{*}{ New Zealand } & \multirow{6}{*}{ Northland } & -35.71373 & 174.32422 \\
\hline & & -35.26022 & 174.13031 \\
\hline & & -35.27994 & 174.08925 \\
\hline & & -35.11389 & 173.26183 \\
\hline & & -35.16033 & 173.16240 \\
\hline & & -35.93756 & 173.87558 \\
\hline & \multirow{6}{*}{ Auckland } & -36.926513 & 174.701767 \\
\hline & & -36.93125 & 174.65039 \\
\hline & & -36.930673 & 174.459839 \\
\hline & & -36.95825 & 174.46911 \\
\hline & & -36.86642 & 174.77161 \\
\hline & & -36.86578 & -174.90233 \\
\hline & Morrisonville & -37.654810 & 175.537097 \\
\hline & Gisborne & -38.635570 & 178.155994 \\
\hline & \multirow{5}{*}{ Hastings } & -39.63147 & 176.85678 \\
\hline & & -39.63789 & 176.84564 \\
\hline & & -39.62700 & 176.85436 \\
\hline & & -39.60 & 176.74 \\
\hline & & -39.62239 & 176.81956 \\
\hline & \multirow{2}{*}{ Wellington } & -40.851118 & 174.993284 \\
\hline & & -41.221639 & 174.871690 \\
\hline & \multirow{4}{*}{ Nelson } & -41.296477 & 173.228223 \\
\hline & & -41.20392 & 173.35661 \\
\hline & & -41.26042 & 173.27583 \\
\hline & & -41.340860 & 173.180681 \\
\hline & \multirow{2}{*}{ Christchurch } & -43.53544 & 172.63575 \\
\hline & & -43.53636 & 172.56286 \\
\hline \multirow{2}{*}{ Australia } & Melbourne & -38.385441 & 144.791506 \\
\hline & Adelaide & -34.909351 & 138.564037 \\
\hline \multirow{3}{*}{ Argentina } & Rosario & -32.910299 & -60.675676 \\
\hline & Otamenti & -34.222211 & -58.896155 \\
\hline & Buenos Aires & -34.543800 & -58.493145 \\
\hline
\end{tabular}




\section{Chapter 5: additional results - RNA Illumina sequencing results}

Table V.1: BLASTx similarity statistics for RNA contigs matching viruses in Argentine ant sample in New Zealand with the best hit viruses. Best hit viruses do not imply RNA contig identity.

\begin{tabular}{|c|c|c|c|c|c|c|c|c|}
\hline $\begin{array}{l}\text { Virus best hit } \\
\text { name }\end{array}$ & Contig ID & Contig Length (bp) & Score & e-value & $\begin{array}{l}\text { \% query } \\
\text { identity }\end{array}$ & $\begin{array}{l}\text { \% query } \\
\text { coverage }\end{array}$ & $\begin{array}{c}\text { Best hit } \\
\text { accession \# }\end{array}$ & Gene $^{a}$ \\
\hline \multirow{9}{*}{ IAPV } & 6805 & 1328 & 385 & 3.E-16 & 59 & 74 & ACD01403 & $\mathrm{nsP}$ \\
\hline & 1094 & 3217 & 651 & $0 \mathrm{E}+00$ & 42 & 91 & YP_008888535 & $\mathrm{nsP}$ \\
\hline & 1028 & 1571 & 571 & 0 & 76 & 67 & ACD01399 & nsP \\
\hline & 1000 & 2564 & 571 & $4 \mathrm{E}-177$ & 76 & 41 & ACD01399 & nsP \\
\hline & 6399 & 524 & 122 & $6 \mathrm{E}-31$ & 43 & 79 & ACDY05306 & capsid \\
\hline & 15309 & 410 & 176 & $8 \mathrm{E}-48$ & 67 & 99 & ABY57949 & $\mathrm{nsP}$ \\
\hline & 24044 & 579 & 249 & 1E-72 & 60 & 100 & ACD01403 & nsP \\
\hline & 17325 & 177 & 50.8 & $1 \mathrm{E}-05$ & 64 & 99 & YP_008888535 & nsP \\
\hline & 30928 & 149 & 75.1 & $5 \mathrm{E}-14$ & 71 & 98 & YP_008888535 & nsP \\
\hline \multirow{14}{*}{ KBV } & 8390 & 434 & 254 & $5 \mathrm{E}-75$ & 98 & 99 & NP_851403 & nsP \\
\hline & 6409 & 1378 & 393 & $8 \mathrm{E}-124$ & 46 & 92 & NP_851404 & stpoly \\
\hline & 1905 & 577 & 388 & $2 \mathrm{E}-121$ & 96 & 99 & NP_851403 & nsP \\
\hline & 2445 & 358 & 241 & $3 \mathrm{E}-76$ & 99 & 99 & AAT76528 & stpoly \\
\hline & 9370 & 398 & 263 & $2 \mathrm{E}-78$ & 98 & 99 & NP_851403 & nsP \\
\hline & 16939 & 229 & 160 & $1.00 \mathrm{E}-47$ & 100 & 99 & AAG28397 & RNApol \\
\hline & 1345 & 1103 & 348 & 2E-104 & 99 & 44 & AHL83499 & nsP \\
\hline & 7337 & 287 & 172 & $4 \mathrm{E}-47$ & 95 & 99 & NP_851403 & nsP \\
\hline & 14185 & 240 & 119 & $6 \mathrm{E}-29$ & 97 & 72 & NP_851403 & $\mathrm{nsP}$ \\
\hline & 11303 & 385 & 259 & $4 \mathrm{E}-83$ & 98 & 98 & AAZ14864 & nsP \\
\hline & 2023 & 518 & 316 & $5 E-96$ & 96 & 93 & AHL83499 & $\mathrm{nsP}$ \\
\hline & 10394 & 235 & 166 & $3 \mathrm{E}-45$ & 100 & 99 & AHL83499 & nsP \\
\hline & 1349 & 453 & 316 & $2 \mathrm{E}-96$ & 99 & 99 & NP_851403 & nsP \\
\hline & 9644 & 508 & 350 & $2 \mathrm{E}-108$ & 99 & 99 & NP_851403 & nsP \\
\hline \multirow{5}{*}{ SINV-2 } & 1050 & 1878 & 452 & $6 \mathrm{E}-137$ & 50 & 72 & YP_001285729 & $\mathrm{nsP}$ \\
\hline & 9293 & 374 & 75.9 & $2 \mathrm{E}-14$ & 42 & 71 & YP_001285726 & stpoly $^{\mathrm{b}}$ \\
\hline & 1841 & 2933 & 598 & $0 \mathrm{E}+00$ & 36 & 97 & YP_001285729 & nsP \\
\hline & 4046 & 257 & 100 & $3 \mathrm{E}-22$ & 53 & 99 & YP_001285729 & nsP \\
\hline & 28325 & 227 & 134 & $3 \mathrm{E}-34$ & 77 & 96 & YP_001285729 & nsP \\
\hline
\end{tabular}

on-structural protein; stpoly, structural polyprotein; RNApol, RNA polymerase.

${ }^{\mathrm{b}}$ putative structural protein. 